\title{
Analysis of Clp1-dependent UPR modulation in Ustilago maydis
}

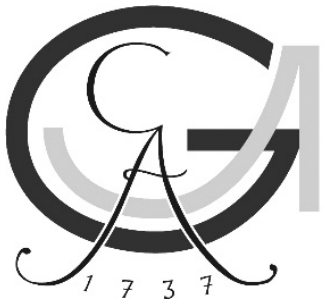

\section{GEORG-AUGUST-UNIVERSITÄT GÖTTINGEN}

Dissertation

for the award of the degree

“'Doctor rerum naturalium”

Division of Mathematics and Natural Sciences

at the Georg-August-University Göttingen

within the doctoral degree program biology

of the Georg-August University School of Science (GAUSS)

submitted by

Niko Pinter

from Achern

Göttingen, 2019 


\section{Thesis committee:}

apl. Prof. Dr. Kai Heimel

Department of Molecular Microbiology and Genetics, Georg-August-Universität Göttingen

Prof. Dr. Gerhard H. Braus

Department of Molecular Microbiology and Genetics, Georg-August-Universität Göttingen

\section{Members of the examination board:}

Referee: $\quad$ apl. Prof. Dr. Kai Heimel

Department of Molecular Microbiology and Genetics, Georg-August-Universität Göttingen

$2^{\text {nd }}$ referee: Prof. Dr. Gerhard H. Braus

Department of Molecular Microbiology and Genetics, Georg-August-Universität Göttingen

\section{Further members of the examination board:}

Prof. Dr. Stefanie Pöggeler

Department of Genetics of Eukaryotic Microorganisms, Georg-August-Universität Göttingen

Prof. Dr. Rolf Daniel

Department of Genomic and Applied Microbiology, Georg-August-Universität Göttingen

PD Dr. Marcel Wiermer

Department of Molecular Biology of Plant-Microbe Interactions, Georg-August-Universität Göttingen

PD Dr. Michael Hoppert

Department of General Microbiology, Georg-August-Universität Göttingen

Date of oral examination: 06.06.2019 


\section{Declaration}

I hereby declare that the thesis entitled "Analysis of Clp1-dependent UPR modulation in Ustilago maydis" was written on my own and independently without any other aids and sources than indicated.

Niko Pinter

Göttingen, 2019 
This work was accomplished in the group of apl. Prof. Dr. Kai Heimel from April 2015 to April 2019, at the Department of Molecular Microbiology and Genetics at the Institute of Microbiology and Genetics, Georg-August University Göttingen.

Parts of this work are published in PLoS pathogens:

Pinter, N.; Hach, CA.; Hampel, M.; Rekhter, D.; Zienkiewicz, K.; Feussner, I.; Poehlein, A.; Daniel, R.; Finkernagel, F.; Heimel, K. (2019): Signal peptide peptidase activity connects the unfolded protein response to plant defense suppression by Ustilago maydis. PLoS pathogens 15 (4), e1007734. DOI: 10.1371/journal.ppat.1007734.

Parts of this work were generated in a supervised master thesis by Hach (2018). Used data of this master thesis were denoted in the figure descriptions of the present study. 


\section{Table of contents}

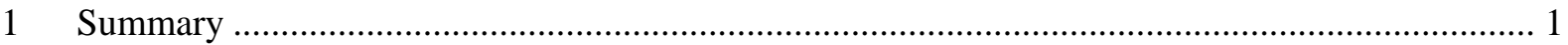

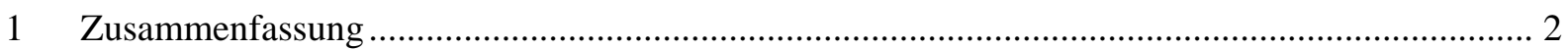

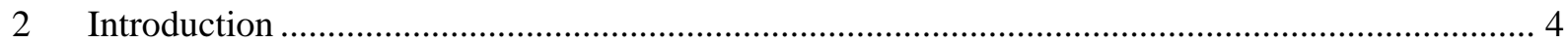

2.1 Ustilago maydis, the causative agent of corn smut disease .................................................. 4

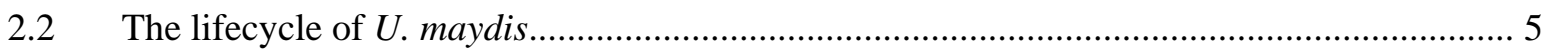

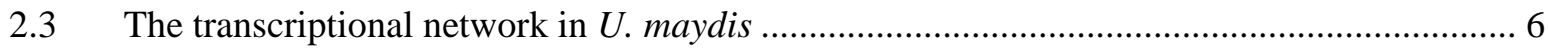

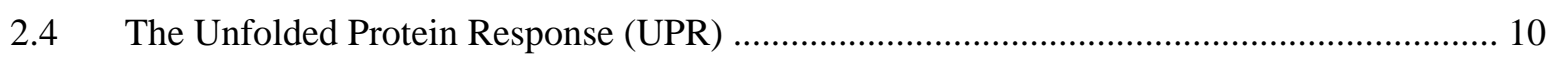

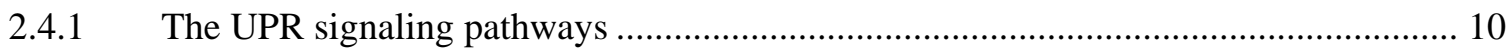

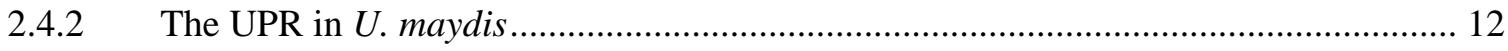

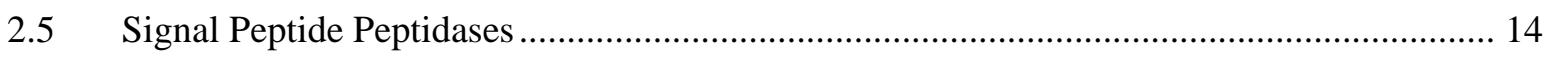

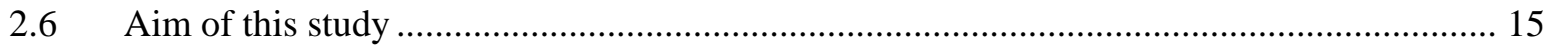

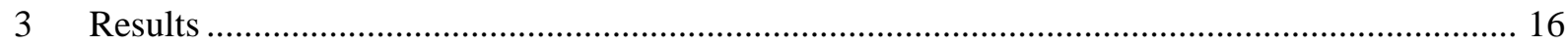

3.1 Functional consequences of the interaction between Cib1 and Clp1 ................................ 16

3.1.1 Fluorescence microscopy of Cib1-GFP strains revealed Clp1-dependent impact on subcellular localization of the fusion protein ........................................................................... 17

3.1.2 Induced expression of Clp1 leads to increased abundance of Cib1 protein and decreased

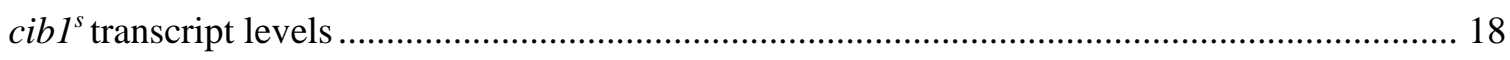

3.1.3 Clp1 expression increases Cib1 protein stability ........................................................... 19

3.1.4 Cib1 is a phosphoprotein and phosphorylation is reduced in strains expressing Clp1.. 21

3.1.5 LC-MS analysis confirms Cib1 phosphorylation and reveals altered phosphorylation

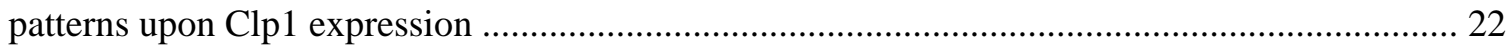

3.2 Consequences of Clp1 expression on UPR gene regulation............................................. 24

3.2.1 RNAseq analysis identifies a set of UPR core genes .................................................. 25

3.2.2 RNAseq reveals modulation of UPR core genes by Clp1 …................................... 27

3.2.3 Clp1 is dispensable for Cib1 DNA binding specificity ................................................ 29

3.3 Deletion of UPR core genes identifies a novel pathogenicity factor................................ 33

3.3.1 Deletion of unrepressed UPR genes had no major impact on pathogenicity and ER stress resistance 33

3.3.2 The UPR regulated gene UMAG_02729 encodes a Signal Peptide Peptidase 
3.3.3 Spp1 is localized to the perinuclear and cortical ER ...................................................... 39

3.3.4 spp1 is involved in the biotrophic growth in planta .................................................... 40

3.3.5 The $\Delta$ spp1 phenotype can be suppressed by expression of Spp1 orthologs .................. 43

3.3.6 Spp1 represses defense responses in planta .................................................................... 46

3.3.7 Deletion mutants of ER-associated degradation pathway (ERAD) and sterol biosynthesis regulator, Srb1, are not impaired in virulence ........................................................................... 50

3.3.8 Effector secretion is not affected in $\Delta$ spp1 strains ...................................................... 54

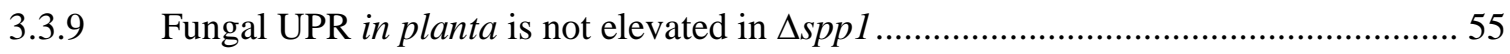

3.3.10 Deletion of UPR elements (UPRE) in the spp1 promoter abolishes UPR-dependent

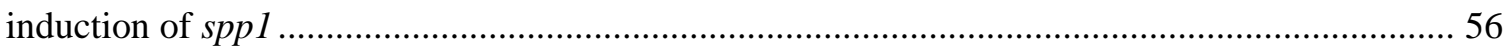

3.3.11 Bioinformatic prediction of UPR elements in SPP promoters ...................................... 59

3.3.12 LC-MS analysis identifies potential Spp1 interaction partners .................................... 60

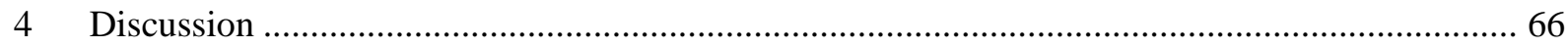

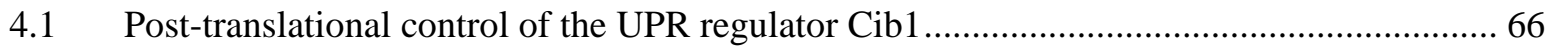

4.1.1 Cib1 mutual stabilize each other Clp1 upon interaction................................................ 66

4.1.2 Cib1 phosphorylation is reduced in a Clp1-dependent manner...................................... 67

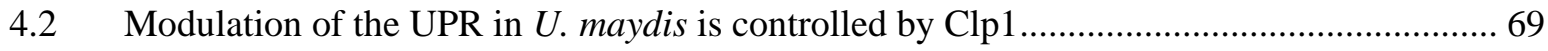

4.2.1 Clp1 is modulating the transcriptional output of the UPR ..........................................69

4.2.2 Clp1 does not alter DNA binding of Cib1 …................................................................ 70

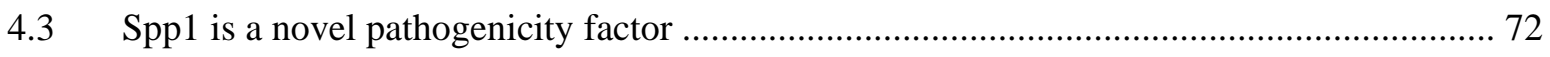

4.3.1 Deletion of UPR genes modulated by Clp1 induction revealed Spp1 as a novel virulence

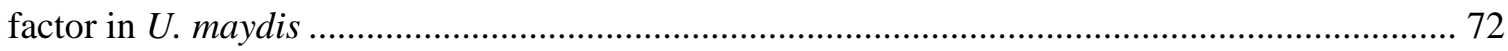

4.3.2 Spp1 supports the establishment of the biotrophic interaction in planta........................ 74

4.3.3 Spp1 is a direct Cib1 target with functional UPREs in its promoter ............................ 76

4.3.4 UMAG_02578 is a potential Spp1 interaction partner identified by LC-MS analysis.. 78

4.4 Model of the Clp1-dependent modulation of the UPR....................................................... 81

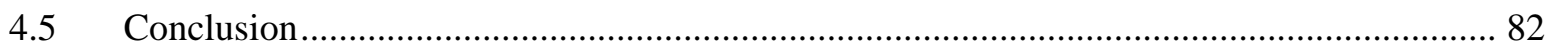

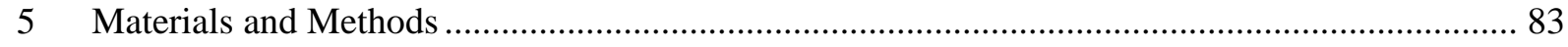

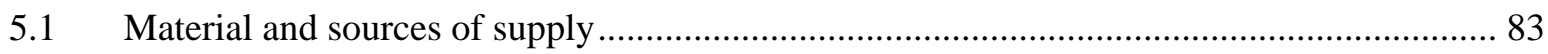

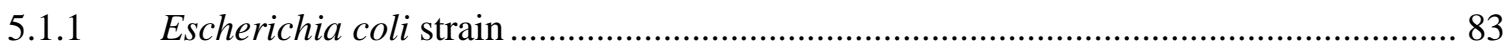




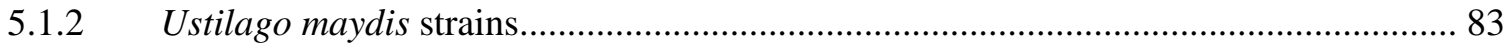

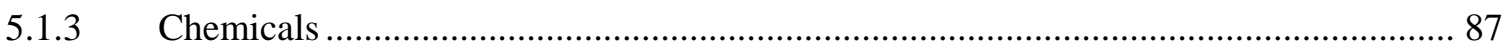

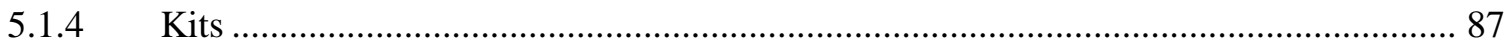

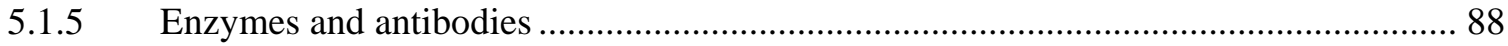

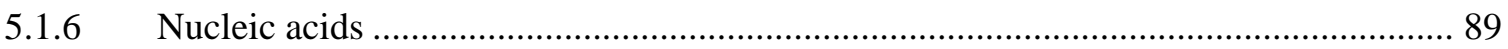

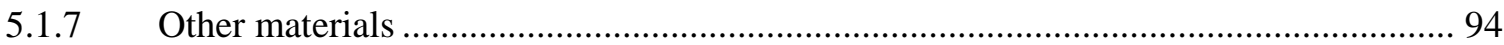

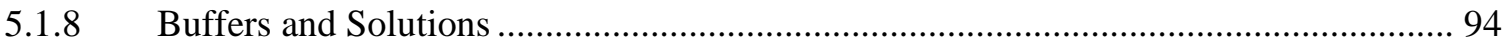

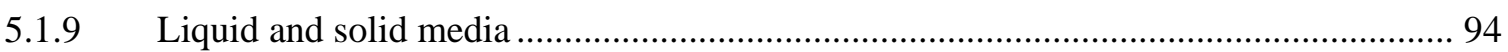

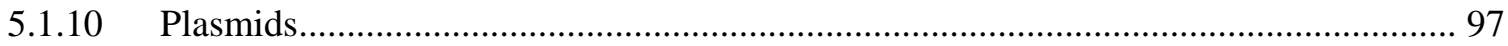

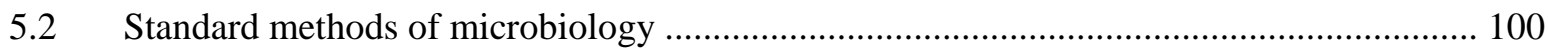

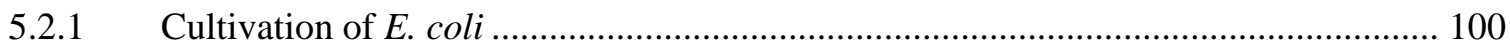

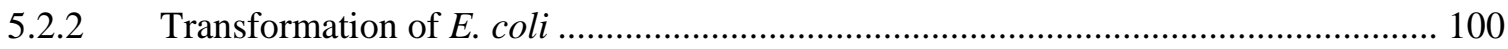

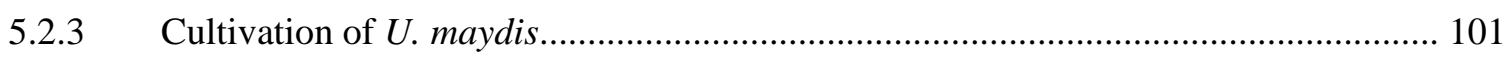

5.2.4 Measurement of cell density in U. maydis ................................................................. 101

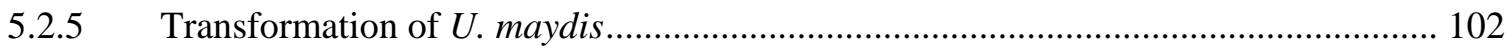

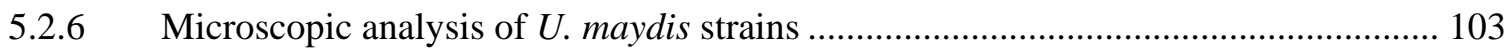

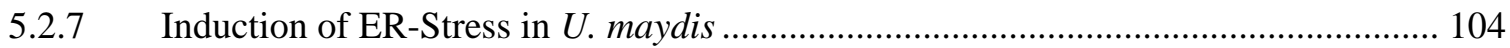

5.2.8 Infection of Zea mays with U. maydis ...................................................................... 104

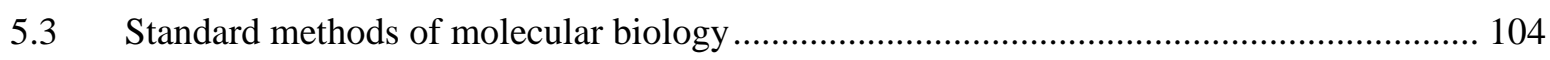

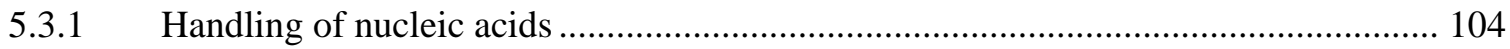

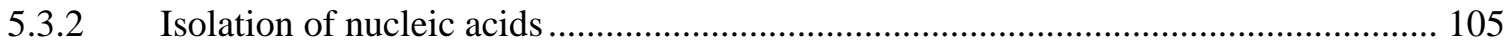

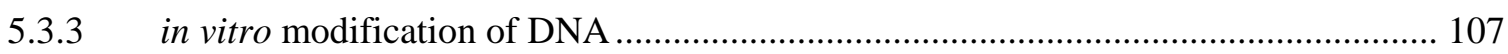

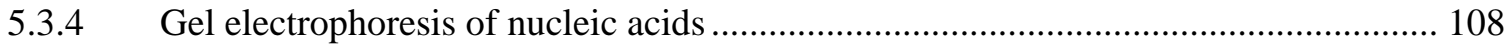

5.3.5 Transfer and detection of DNA on membranes (Southern-Blot)................................. 109

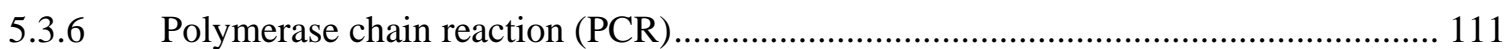

5.3.7 Quantitative reverse transcription-PCR (qRT-PCR) ................................................... 112

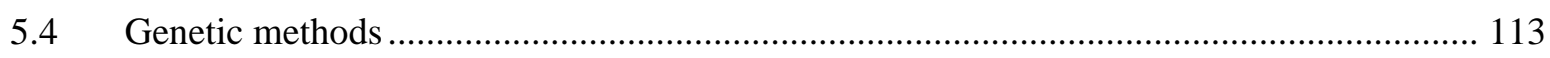

5.4.1 PCR amplification of gene deletion and fusion constructs for $U$. maydis.................... 113

5.4.2 Integration of constructs in the ip locus of $U$. maydis ................................................ 113

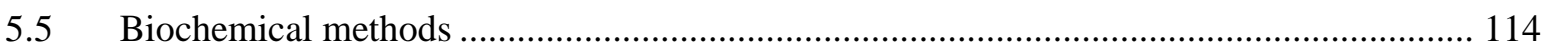


5.5.1 Protein extraction of $U$. maydis for protein analyses.................................................. 114

5.5.2 SDS polyacrylamide gel electrophoreses of proteins ................................................ 115

5.5.3 Detection of immobilized proteins (Western-Blot) .................................................. 116

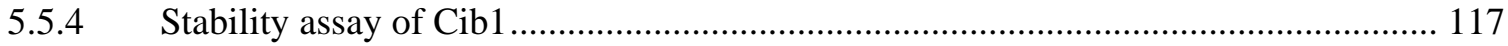

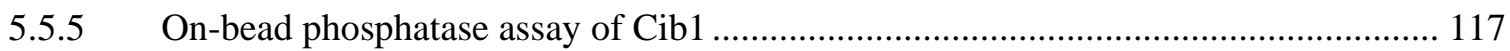

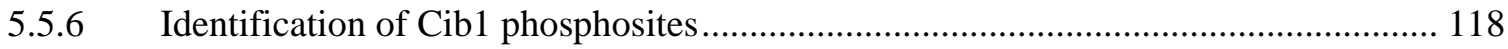

5.5.7 Immunoprecipitation of Spp1-GFP / Spp1 ${ }^{\text {D279A }}$-GFP in U. maydis .............................. 118

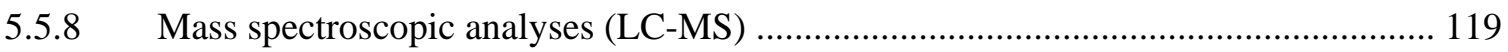

5.6 Whole-genome sequencing approaches …...................................................................... 120

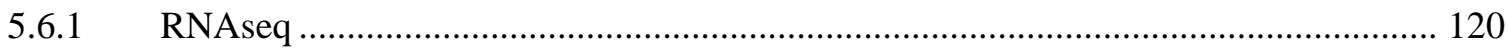

5.6.2 Chromatin immunoprecipitation sequencing (ChIPseq) .............................................. 121

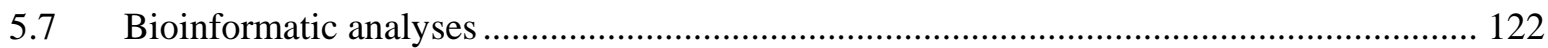

5.7.1 Sequencing of DNA and plasmids used for cloning.................................................. 122

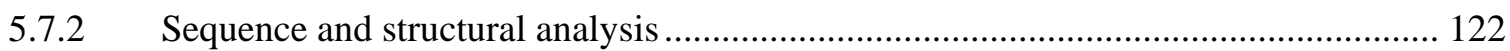

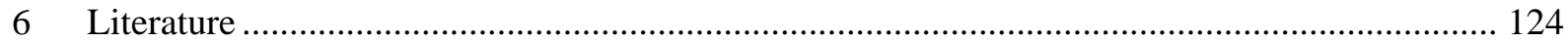

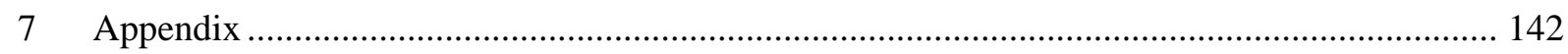

7.1.1 ChIPseq analysis revealed Cib1 binding in tin1-1 promoter ..................................... 142

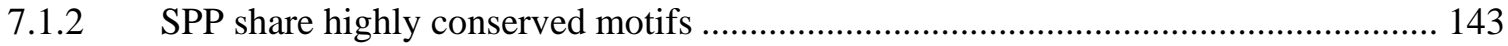

7.1.3 $\Delta$ spp1 led to increased expression of PR3 and PR4 in planta ….................................. 144

7.1.4 $\quad \Delta$ spp1 strains are not impaired in cell wall stress ..................................................... 145

7.1.5 Coomassie staining of the Cib1 protein in an SDS-polyacrylamide gel ...................... 145

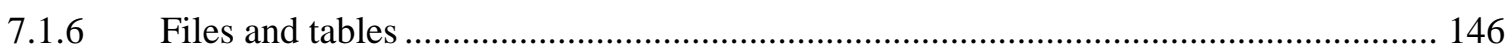

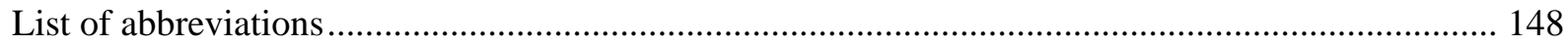

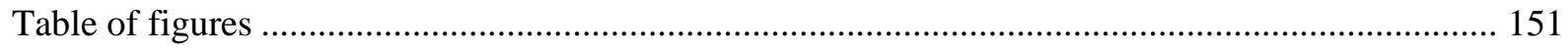

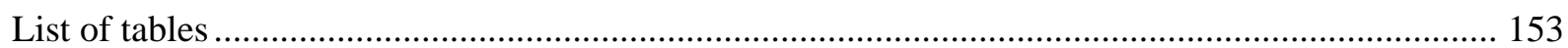




\section{Summary}

The unfolded protein response (UPR) is a conserved signaling pathway, that is present in all eukaryotic cells and ensures endoplasmic reticulum (ER) homeostasis under stress conditions. In the phytopathogenic fungus $U$. maydis, the UPR is activated after plant penetration as a result of increased demands on the secretory pathway during the fungal/plant interaction. However, prolonged activation of the UPR is deleterious for U. maydis and UPR activity needs to be modulated during plant colonization. This modulation is achieved by the physical interaction between Cib1 (Clp1 interacting bZip 1), the central regulator of the UPR and Clp1 (Clampless 1 ), an important developmental regulator of $U$. maydis and the decisive factor for the induction of fungal proliferation after successful host penetration. The interaction between both proteins leads to increased stability of Clp1 and alters UPR gene expression. In this study, the functional consequences of this interaction on the physical properties of Cib1, the impact on Cib1 DNA binding and the transcriptional output of the UPR were characterized. Expression of clp1 leads to elevated ER stress resistance, increased protein stability and altered phosphorylation patterns of Cib1. Transcriptome analysis (RNAseq) during ER stress identified a set of 65 upregulated UPR core genes, whose expression is differentially modulated upon clp1 induction. Chromatin immunoprecipitation of Cib1 with subsequent whole-genome sequencing (ChIPseq) identified UPR elements (UPRE) in promoters of the large majority of UPR core genes and revealed that Cib1 DNA-binding specificity is not altered by Clp1. In a comprehensive gene deletion analysis, a previously uncharacterized UPR target gene was identified that is specifically required for biotrophic growth of $U$. maydis. UMAG_02729 encodes an intramembrane cleaving signal peptide peptidase (spp1) that contains a conserved active site typical for aspartyl proteases. Plants inoculated with $\Delta$ spp1 mutants or strains expressing enzymatically inactive Spp1 triggered massive plant defense responses as evidenced by reactive oxygen species (ROS) accumulation and strongly increased expression of pathogenesis-related plant genes. Complementation of the spp1 deletion strain with orthologous genes from Sporisorium reilianum and Ustilago hordei recovered virulence and expression of the wellcharacterized human ortholog HM13 suppressed the virulence defect of the spp1 deletion mutant in a dose-dependent manner. However, the virulence-specific function of Spp1 is not related to known functions of signal peptide peptidases, such as ER-associated degradation (ERAD), hypoxia adaptation or effector secretion. Deletion of predicted UPREs in the promoter of spp1 significantly reduced spp1 expression upon ER stress. Co-immunoprecipitation analysis of Spp1 with subsequent LC-MS analysis revealed members of the signal peptidase complex (SPC) and the O-mannosyltransferase Pmt4, as potential interaction partners or substrates of Spp1. In summary, the data of this study revealed a potential mechanism on how UPR in U. maydis is modulated by Clp1 and a novel factor important for plant defense suppression that is not connected to previously known pathways related to signal peptide peptidase function or plant defense suppression by phytopathogenic fungi. 


\section{Zusammenfassung}

Die unfolded protein response (UPR) ist ein konservierter Signalweg, welcher in allen eukaryotischen Zellen vorkommt und die Homöostase des endoplasmatischen Retikulums unter Stressbedingungen aufrechterhält. In dem phytopathogenen Pilz Ustilago maydis wird die UPR nach der Penetration der Pflanze, aufgrund eines erhöhten Bedarfs des sekretorischen Signalwegs während der Pilz/PflanzenInteraktion, aktiviert. Eine andauernde Aktivierung der UPR ist schädlich für U. maydis, weshalb die UPR Aktivität während der Pflanzenkolonisation moduliert werden muss. Diese Modulation findet durch die physische Interaktion zwischen dem zentralen UPR Regulator (ㄷlp1 interacting $\underline{b} Z$ IP $\underline{1}$ ) und dem wichtigen Entwicklungsregulator Clp1 (lampless 1 ) statt. Clp1 ist ein entscheidender Faktor für das Auslösen der pilzlichen Vermehrung nach der Wirtspenetration. Die Interaktion beider Proteine führt zu einer erhöhten Stabilität von Clp1 und verändert die Expression von UPR Markergenen. In der vorliegenden Arbeit wurden die funktionellen Auswirkungen dieser Interaktion auf die physischen Eigenschaften von Cib1, die Cib1 DNA Bindung und die Transkription der UPR untersucht. Die Expression von clp1 führt zu einer erhöhten ER Stresstoleranz sowie einer erhöhten Proteinstabilität und eines veränderten Phosphorylierungsmusters von Cib1. In einer Transkriptomanalyse (RNAseq) unter erhöhtem ER Stressbedingungen konnte eine Gruppe von 65 hochregulierten UPR Hauptgenen identifiziert werden, deren Expression während der clp1 Induktion differenziell moduliert ist. Eine Chromatin-Immunopräzipitationsanalyse von Cib1 mit anschließender Sequenzierung (ChIPseq) identifizierte UPR-Elemente (UPRE) mit gehäuftem Vorkommen in Promotoren der UPR Hauptgene. Die DNA-Bindungsspezifität von Cib1 durch die Clp1 Induktion bleibt jedoch unverändert. In einer umfassenden Gendeletionsanalyse konnte ein zuvor nicht charakterisiertes UPR-Zielgen identifiziert werden, das speziell für das biotrophe Wachstum von U. maydis in der Pflanze erforderlich ist. UMAG_02729 codiert für eine intramembranspaltende Signalpeptid-Peptidase (spp1), welche ein konserviertes, aktives Zentrum aufweist, das typisch für Aspartylproteasen ist. Pflanzen, die mit $\Delta s p p 1$ Mutanten oder Stämmen infiziert wurden, die ein enzymatisch inaktives Spp1 exprimierten, lösten starke Pflanzenabwehrreaktionen aus, die durch die Akkumulation von reaktiven Sauerstoffspezies (ROS), sowie einer erhöhten Expression von Pflanzengenen der Pathogenese nachgewiesen werden konnte. Die Komplementierung des spp1-Deletionsstamms mit orthologen Genen aus Sporisorium reilianum und Ustilago hordei konnte die verlorene Virulenz vollständig wiederherstellen. Zusätzlich konnte gezeigt werden, dass die Expression des gut charakterisierten, humanen Orthologs HM13 den Virulenzdefekt der spp1-Deletionsmutante dosisabhängig unterdrückt. Allerdings steht die virulenzspezifische Funktion von Spp1 nicht im Zusammenhang mit bekannten Funktionen von Signalpeptidpeptidasen, wie beispielsweise der ER-assoziierten Degradation (ERAD), der Anpassung an Hypoxie oder der Effektorsekretion. Die Deletion vorhergesagter UPREs im Promotor von spp1 reduzierte die spp1-Expression bei ER-Stress signifikant. Die Co-Immunopräzipitation von Spp1 mit anschließender LC-MS Analyse brachte Mitglieder des Signalpeptidase-Komplexes (SPC) und der OMannosyltransferase Pmt4 als mögliche Interaktionspartner oder Substrate von Spp1 hervor. 
Zusammenfassend zeigen die Daten dieser Studie einen potenziellen Mechanismus, wie die UPR in U. maydis durch Clp1 moduliert werden könnte. Zudem konnte ein neuer Faktor identifiziert werden, der für die Unterdrückung der Pflanzenabwehr wichtig ist und nicht mit den bisher bekannten Signalwegen der Signalpeptidpeptidasefunktion oder der Unterdrückung der Pflanzenabwehr durch phytopathogene Pilze zusammenhängt. 


\section{Introduction}

\subsection{Ustilago maydis, the causative agent of corn smut disease}

Ustilago maydis is a phytopathogenic basidiomycete, which infects its host plant maize (Zea mays) as well as its wild progenitor teosinte (Z. mays ssp. parviglumis and ssp. mexicana) (Doebley, 1992). Systematically, U. maydis belongs to the phylum Basidiomycota, in the class of Ustilaginomycetes (true smut fungi) of the order of Ustilaginales (smut fungi) (Lutzoni et al., 2004). Initial disease symptoms of
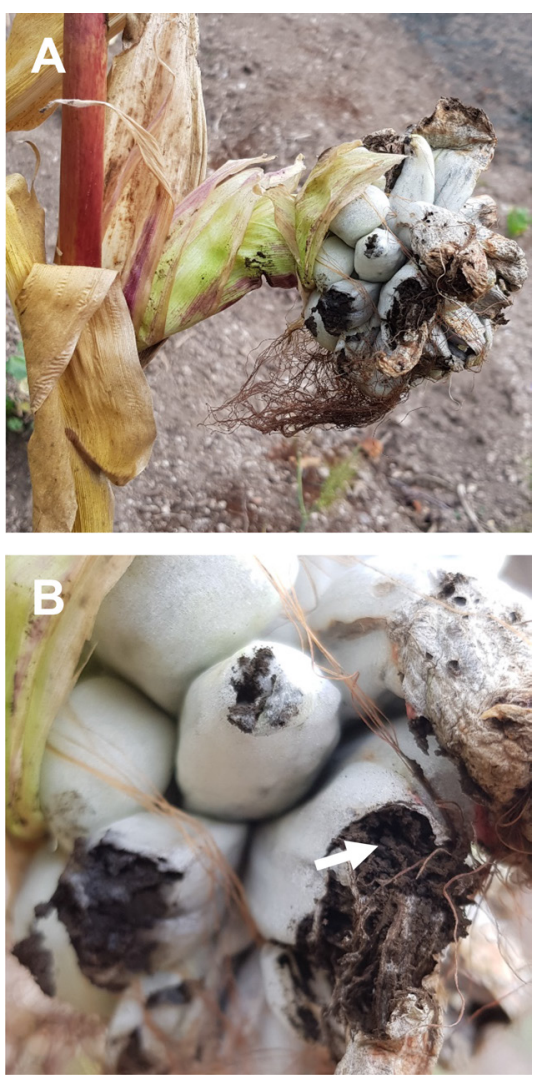

Figure 2.1: Corncob infected with U. maydis. (A) Each of the white galls are developing out of a single grain. Galls can be formed out of other plant tissue, like leaf and stem tissue. (B) Bursted galls release the melanized teliospores (arrow).

U. maydis are primarily reflected by increased anthocyanin production and chlorosis formation in the leaf tissue of its host plant. Subsequently, infected plants form the characteristic tumorlike galls at all above-ground parts of the plants including the corncob, leaves, and stem (Fig 2.1A). The fungus is edible, and especially in Mexican cuisines, the galls of a U. maydis-infected corncob are considered as a delicacy, also known as "Huitlacoche" or the "Mexican truffle". Bursting of these white galls at the end of its lifecycle causes the release and dispersal of their black melanized teliospores (Fig 2.1B, white arrow). This gives the maize plant a burned appearance, which is eponymous for Ustilago, from the Latin verb ustilare (to burn). U. maydis is considered as an agricultural pest, although yield loss due to corn smut in cultivated maize plants is limited to about $2 \%$ by the use of resistant maize varieties (Munkvold and White, 2016). U. maydis has become one of the most important model organisms for phytopathogenic fungi in recent decades. In 1964, Robert Holliday described the basic model of homologous recombination, also known as the Holliday structure, in U. maydis (Holliday, 1964). Sequencing and publishing of the approximately $20 \mathrm{Mb}$ sized genome in 2006, enabled reverse genetic approaches (Kämper et al., 2006). Transcriptional profiling of the entire plant-associated development of U. maydis in 2018 provided new insights on gene regulation during in planta development on a whole-genome level (Lanver et al., 2018). $U$. maydis has a dimorphic life cycle, including a saprophytic, non-pathogenic phase and a biotrophic, pathogenic phase. Under laboratory conditions, the life cycle of $U$. maydis can be completed in three to four weeks. Genetic manipulations are possible in its saprophytic, haploid form in which the fungus can be easily cultivated on solid and liquid media. In addition, $U$. maydis is highly amenable to modification using molecular genetic standard methods and a highly efficient homologous recombination (Kämper, 2004). 


\subsection{The lifecycle of $U$. maydis}

U. maydis is a phytopathogenic fungus that depends on its host plant maize to fulfill its lifecycle. The lifecycle can be divided into two phases, the saprophytic and biotrophic phase. In the saprophytic phase, cells are haploid and have a yeast-like growth (Fig 2.2A). On the plant surface, two haploid sporidia form conjugation tubes towards a pheromone gradient (Fig 2.2B), if both cells have compatible matingtype loci (Banuett, 1995). Sensing of a compatible pheromone leads to cell cycle arrest in the G2 phase. (García-Muse et al., 2003; Sgarlata and Pérez-Martín, 2005). U. maydis enters the biotrophic phase of its lifecycle, after fusion of the conjugation tubes, that leads to the formation of the infectious dikaryon, which grows as a filament (Fig 2.2C). Plant penetration is mediated by developing the appressorium (Fig 2.2D), a specialized fungal infection structure (Snetselaar and Mims, 1993).

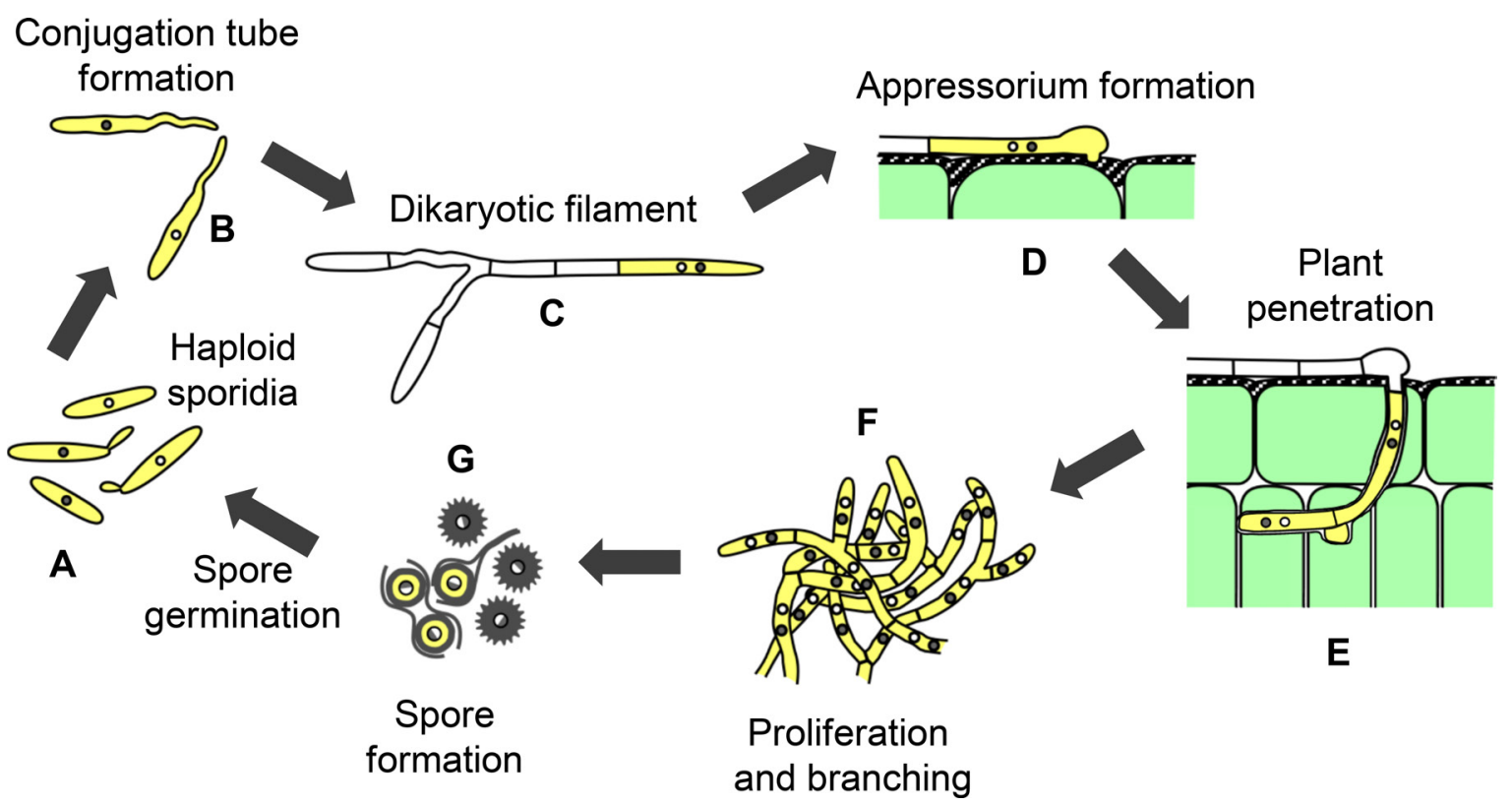

Figure 2.2: Lifecycle of Ustilago maydis. (A) Haploid, heterothallic sporidia (B) Two sporidia with compatible mating-type loci forming a conjugation tube towards a pheromone gradient (C) Fusion of both sporidia led to the formation of the dikaryotic filament (D) Formation of the appressorium (E) Penetration of the plant surface and growth in planta $(\mathbf{F})$ Proliferation and branching in planta (G) Spore formation and germination (modified after (Kämper et al., 2006; Heimel et al., 2010a))

During plant penetration, the plasma membrane of its host plant surrounds the invading hypha, which grows between the inter- and intracellular plant tissue (Fig 2.2E) (Snetselaar and Mims, 1993). The invagination of the plasma membrane creates an interaction zone between the fungus and the plant, where fungal secretion of effector proteins dampens the host's plant defense (Lanver et al., 2017). Only after successful penetration, the G2-cell cycle arrest is released and clamp cells are formed, which mediate the proper distribution of the two different nuclei during dikaryotic growth and ensure fungal proliferation in the host plant (Fig 2.2F) (Scherer et al., 2006). After massive proliferation and tumor induction, fungal hyphae undergo karyogamy and start to transform into diploid teliospores (Fig 2.2G) (Banuett and Herskowitz, 1996). The melanized teliospores are dispersed by wind, rain or insect vectors and germinate under suitable environmental conditions. After meiosis, haploid sporidia are released from the basidium by budding, and the life cycle starts all over again (Christensen, 1963). 


\subsection{The transcriptional network in $U$. maydis}

Cell recognition of two compatible sporidia, as well as fusion of both cells, is genetically controlled by the biallelic $a$-locus in $U$. maydis. Genes of the $a$ mating-type locus that encode components for the cellcell recognition exist in the alleles $a 1$ and $a 2$. The $a$-locus contains the genes $m f a 1 / 2$, encoding the pheromone Mfa (mating factor a) and pra1/2, encoding the pheromone receptor Pra (pheromone receptor a). Pheromone recognition during mating occurs when both sporidia possess different $a$-alleles. Thereby, the secreted lipopeptide mating factor is recognized by its cognate pheromone receptor of the other mating type (Bölker et al., 1992). Hence, formation of conjugation tubes is directed towards a pheromone gradient of the other mating type, which leads to the fusion of both conjugation tubes and subsequent formation of the dikaryotic filament (Spellig et al., 1994; Snetselaar et al., 1996). Furthermore, the signal cascade leads to activation of the $b$-locus via the transcription factor Prf1 (Fig 2.4B) (Hartmann et al., 1996).

The multiallelic $b$-locus in $U$. maydis controls the sexual and pathogenic development after fusion of two compatible sporidia. Its activation leads to a G2 cell cycle arrest that is released after plant infection as well as a downregulation of the $a$-pathway. In addition, activation of the $b$-locus is essential for the formation of the heterodikaryon and is crucial for the transition from the saprophytic growth to the biotrophic plant interaction (Schlesinger et al., 1997). The $b$-locus consists of two genes, $b E$ (bEast) and $b W$ (bWest), with a protein length of $473 \mathrm{AA}$ and $644 \mathrm{AA}$, respectively (Fig 2.3A and B). Both genes are divergently transcribed by the same promoter (Fig 2.3A) and encoding for homeodomain proteins, unrelated in sequence (Gillissen et al., 1992). bE and bW can dimerize and form an active homeodomain transcription factor. However, dimerization only occurs, if both proteins originate from different alleles, leading to activation of the subsequent pathogenic development (Fig 2.3C). In contrast to the $a$-locus, which constitutes only two alleles, the $b$-locus consists of at least 19 different $b$ alleles (J. Kämper, unpublished). The $\mathrm{bE}$ and $\mathrm{bW}$ proteins derived from different $b$ alleles mainly differ in their $\mathrm{N}$-terminal domains and intergenic spacer regions, which are highly variable (Fig 2.3B). DNA binding of bE/bW heterodimer is promoted by their homeodomain located in their conserved $\mathrm{N}$-terminus, whereas their $\mathrm{N}$ terminal regions are necessary for dimerization of the heterodimer bE/bW (Kämper et al., 1995).

Sexual and pathogenic development in U. maydis are tightly connected, and both controlled by a complex regulatory network of transcription factors. The generation of solopathogenic strains like CL13 ( $a 1 b W 2 / b E 1$ ) exploited the $b$-pathway by cloning compatible $b$-alleles into one strain. As a result, these strains enable plant infection without a mating partner, and gene deletion studies are effortless in haploid strains used for plant infection (Bölker et al., 1995). However, the virulence of the strain CL13 significantly increased by introducing the $m f a 2$ gene into the strain, to activate the pheromone signaling resulting in the commonly used SG200 (a1 mfa2 bW2/bE1) strain (Kämper et al., 2006). Moreover, the latter demonstrates that the interplay between both pathways is required to gain the full virulence of $U$. maydis during plant infection. 
Formation of the bE/bW heterodimer leads to DNA binding of a conserved motif, b-binding site (bbs), in promoter regions of a small subset of $b$ responsive genes (class 1 genes). Genes of class 1 are primarily involved in the regulation of further downstream targets (class 2 genes). However, 345 genes showed an altered expression upon complex formation of bE and bW. Moreover, most of these genes are important to establish the biotrophic phase, the cell cycle regulation and the polarized growth of the infectious filament (Urban et al., 1996; Heimel et al., 2010b).

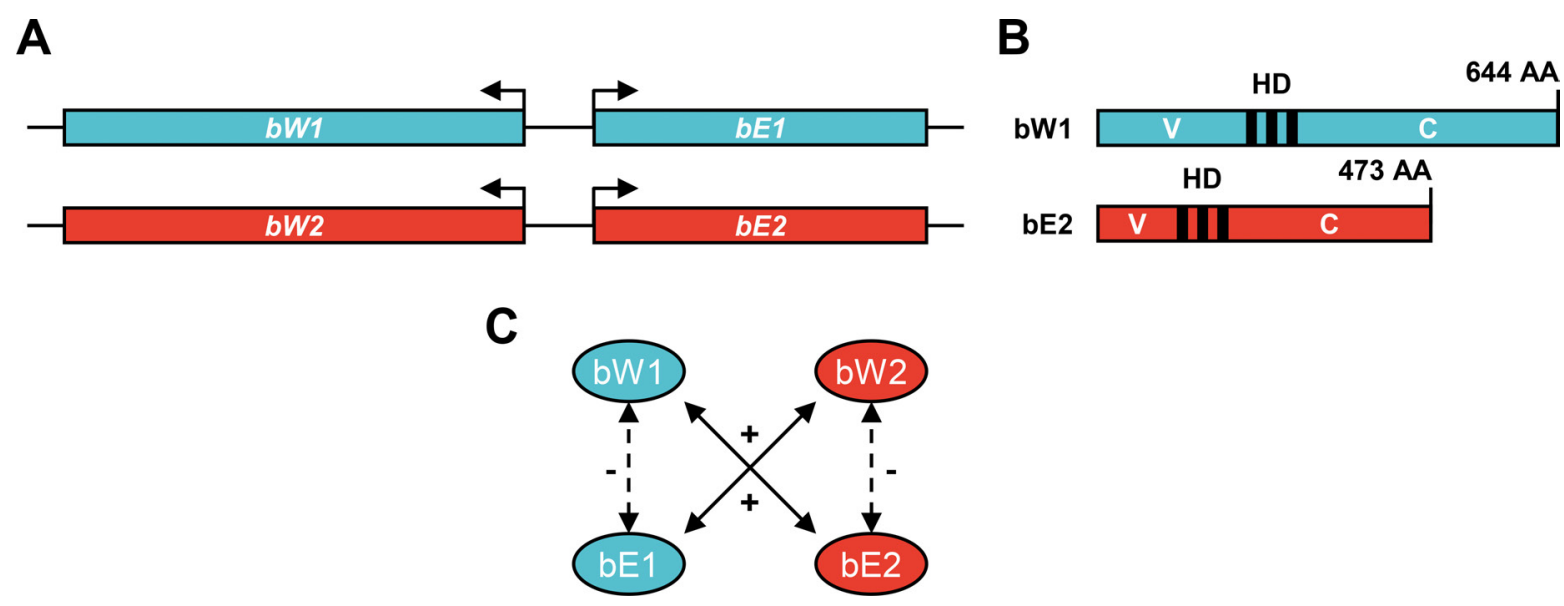

Figure 2.3: Schematic representation of the $\boldsymbol{b}$-locus in $\boldsymbol{U}$. maydis. (A) The multiallelic $b$-locus consists of two genes, $b E$ (bEast) and $b W(b W e s t)$, divergently transcribed by the same promoter. Arrows indicate transcriptional start site. Colors (blue and red) indicate different alleles. (B) Both proteins contain a homeodomain (HD) that promotes DNA binding. In contrast to the conserved, invariable C-terminus (C) of both proteins, the N-terminal domain has a high sequence variability $(\mathrm{V})$ and is involved in dimer formation. Colors (blue and red) indicate proteins derived from different alleles. (C) Formation of the heterodimer only occurs, when $b$ genes are derived from different $b$ alleles. Minus (-) and plus (+) indicate "no interaction possible" or "interaction possible", respectively. Colors (blue and red) indicate proteins derived from different alleles (modified after (Brachmann et al., 2001)).

However, the majority of class 2 genes lack a $b$-binding site in their promoter region that is found in promoters of class 1 genes. The central regulator of the $b$-dependent transcriptional cascade is $r b f 1$ (regulator of $\underline{b}$-filament $\underline{1}$ ), a C2H2 zinc finger transcription factor, is part of the class 1 genes and is required for expression of around $90 \%$ of the $b$-regulated genes. $b$-dependent induction of Rbf1 is required for pathogenic development since deletion of $r b f 1$ abolishes all $b$-mediated processes. The efficient formation of appressoria and the subsequent penetration of the leaf surface is mainly triggered by the zinc finger transcriptions factor Biz1, and the MAP kinase Kpp6, which are both induced by Rbf1 (Heimel et al., 2010b).

Another gene of the class 1 category is clp1 (clampless $\underline{1}$ ). Deletion of clp1 does not affect the growth of haploid cells, appressoria formation or plant invasion. However, after plant penetration, clp1 deletion strains are not able to form clamp cells and cannot proliferate in planta. clp1 has two predicted bbs motifs in its promoter and is one of the few directly $b$-regulated genes. clp1 is rapidly induced after formation of the b-heterodimer. However, the Clp1 protein is detectable only after appressoria formation, by enhanced protein stability of Clp1 via interaction with the regulator of the unfolded protein 
response (UPR), Cib1 (Chapter 2.4.2). Clp1 then interacts with bW and Rbf1, which negatively interferes with the $a$ and $b$-pathway (Fig 2.4C).
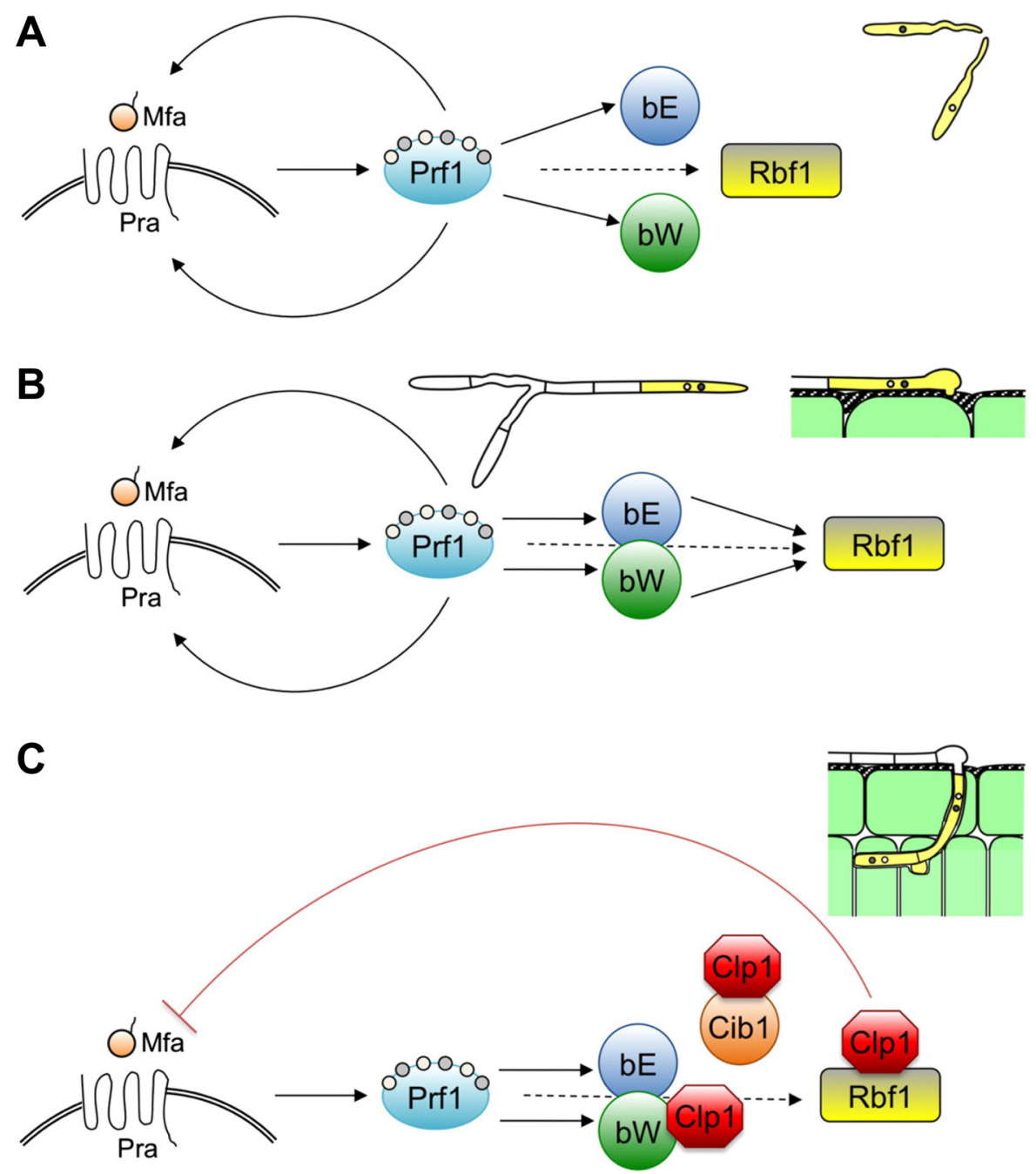

Figure 2.4: Model of the transcriptional network of sexual and pathogenic development in $U$. maydis. (A) Cell recognition of two compatible sporidia mediated by a pheromone (Mfa)/receptor (Pra) system activates a MAPK signal transduction. Activation of this signal transduction triggers the transcription factor Prf1 that regulates genes of the $a$-locus, $m f a$ and pra, as well as genes of the $b$-locus, $b E$ and $b W$. In consequence, activation of the $a$-pathway leads to G2 cell cycle arrest and cell fusion of the pheromone-directed conjugation tubes. (B) Cell fusion of both sporidia leads to the formation of the dikaryotic filament, that is primarily controlled by the interaction of the compatible bE and bW homeodomain proteins, forming an active transcriptions factor. The master regulator of the pathogenic development, $r b f 1$, is upregulated by bE/bW heterodimer. Induction of $r b f 1$ is prerequisite for the establishment of the biotrophic phase, cell cycle regulation and polarized growth of the infectious filament (C) Expression of clp1 is induced after formation of the bE/bW heterodimer. However, the Clp1 protein is only detected after appressoria formation due to increased stability of the protein by binding of Cib1. Higher protein levels of Clp1 then lead to a downregulation of the $a$ - and $b$-pathway by direct protein interaction with Rbf1 and bW, respectively. Repression of both pathways leads to the release of the cell cycle arrest the $a$ - and $b$-pathway have established, which enables activation of further developmental processes (Heimel et al., 2010a; Heimel et al., 2013)

Interaction of Clp1 with bW strongly affects the $b$-pathway by blocking all $b$-function. Furthermore, Clp1 interaction with Rbf1 represses the pheromone pathway, by reducing $m f a 1$ and pra1 expression levels. Moreover, Clp1-mediated reduction of $m f a 1$ expression levels leads to a downregulation of the $b$ genes, since the $a$-pathway is required for expression of $b E$ and $b W$. Hence, repression of both pathways also leads to a drastic downregulation of the complete signaling pathway, which is thought be 
required for the release of the $a$ - and $b$-mediated cell cycle arrest and necessary for the initiation of the further developmental programs after plant penetration. Since clp1 expression is regulated by the $\mathrm{bE} / \mathrm{bW}$ heterodimer, this feedback regulation after plant penetration prevents complete suppression of the signaling pathway and establishes an oscillatory self-perpetuating regulatory network connecting cell cycle control to pathogenic development. (Scherer et al., 2006; Heimel et al., 2010a; Heimel et al., 2013). 


\subsection{The Unfolded Protein Response (UPR)}

After filament formation and penetration of the plant surface, interaction between Clp1 and the central UPR regulator Cib1 increases the stability of the Clp1 protein. Thus, this interaction leads to the release of the $b$-dependent cell cycle arrest and promotes further development in planta. Moreover, the interaction between both proteins leads to elevated ER stress resistance, which might be crucial for efficient secretion of effector proteins in planta (Heimel et al., 2013).

\subsubsection{The UPR signaling pathways}

Most of the secreted and transmembrane proteins in eukaryotic cells enter the ER as unfolded polypeptides. Thus, these polypeptides are folded and post-translationally modified in the endoplasmic reticulum (ER) lumen. However, influx levels of pre-mature proteins can vary upon different developmental stages or environmental changes, affecting the physiological condition of the cell. To cope with an increased secretory demand, cells have to adapt to new conditions and restore the homeostasis of the ER. Thus, cells harbor control mechanisms that continuously monitor the proteinfolding status and adapt intracellular signaling pathways. Imbalances in demand for protein folding in

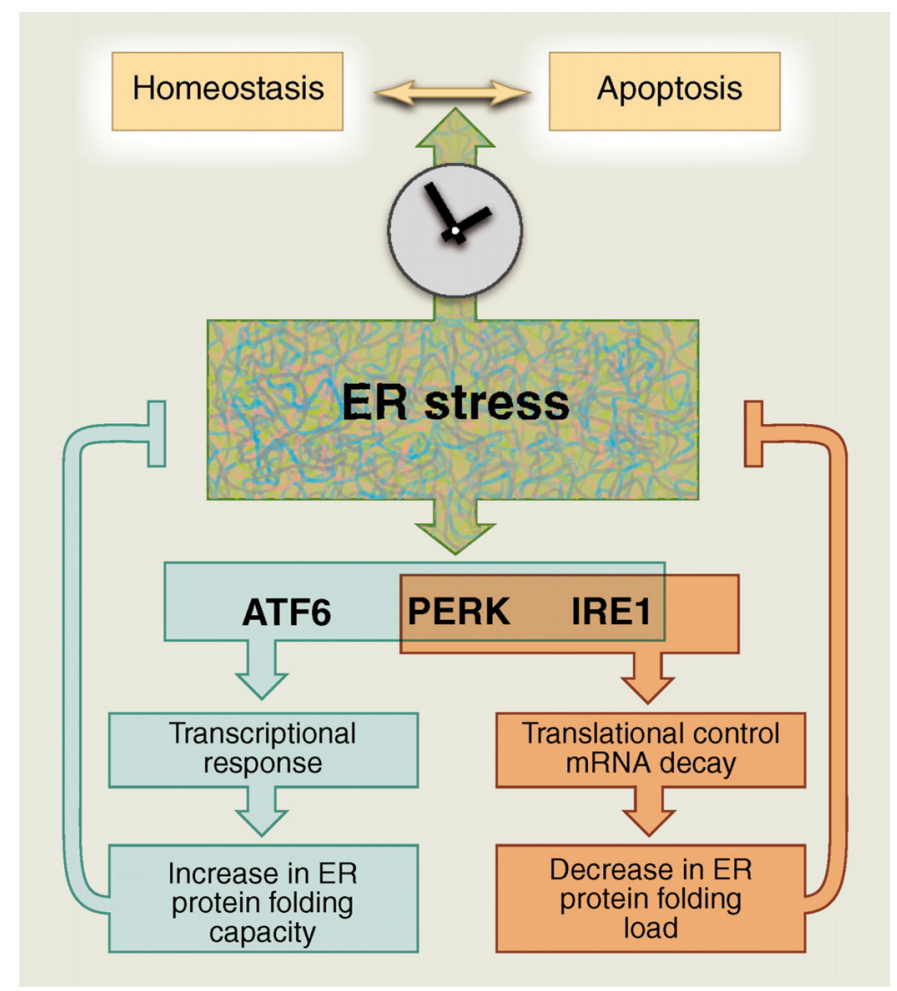

Figure 2.5: Schematic representation of UPR pathways compete with ER stress. The UPR pathways ATF6, PERK and IRE1 are activated upon ER stress to reestablish ER homeostasis. Activation of ATF6 and IRE1 increase the folding capacity of the ER, PERK and IRE1 decrease the level of proteins that enter the ER. All three UPR pathways can be found in metazoans. However, only the IRE1 pathway is conserved among all eukaryotes. Prolonged UPR activity activates cell's apoptosis program (Walter and Ron, 2011). the ER and protein-folding capacity leads to accumulation of unfolded or misfolded proteins in the ER (ER stress) and activation of the unfolded protein response (UPR) (Karagöz et al., 2019). The UPR plays a critical role in restoring the ER homeostasis, by preventing accumulation of potentially toxic proteins caused by an imbalanced protein-folding capacity of the ER. So far, three distinct branches of the UPR have been identified: The PERK (double-stranded RNA-activated protein kinase (PKR)-like ER kinase) and ATF6 (activating transcription factor $\underline{6}$ ) pathway, both only existing in metazoans, as well as the most conserved IRE1 (inositol-requiring enzyme 1) pathway that is present from yeast to mammals (Mori, 2009). In metazoans, each branch is differently represented in different cell tissues. Activation of the PERK, ATF6 or IRE1 pathway leads to the production of 
the bZip transcription factors ATF4, ATF6(N) or XBP1 (ㅁ-box binding protein $\underline{1}$ ), respectively, and activate downstream UPR targets as homo- or heterodimers. UPR target genes are upregulated to decrease the load of proteins entering the ER (IRE1 and PERK) and increase the ER's protein-folding capacity (IRE1 and ATF6). However, if cells cannot reestablish ER homeostasis and suffer from a prolonged UPR activity apoptosis is induced (Tabas and Ron, 2011; Hetz, 2012; Karagöz et al., 2019)

(Fig 2.5).

The best-studied and most conserved branch of the UPR among all eukaryotic cells is the Ire1 pathway (Fig 2.6). In S. cerevisiae, Ire1p is a single-pass ER transmembrane protein, consisting of a kinase and nuclease domain at its cytoplasmic region and an ER luminal domain of Ire1p that senses unfolded or misfolded proteins. Binding of un- or misfolded proteins leads to oligomerization and transautophosphorylation of Ire1p accompanied by a conformational change of its protein structure. Oligomerization and structural changes of Ire1 facilitate activation of its RNase domain, which catalyzes unconventional splicing of the HAC1 mRNA (Gardner and Walter, 2011). The unconventional splicing event is highly specific, since Ire1p only excising the intron out of the mRNA that encodes the UPR transcription factor XBP1 in metazoans (Yoshida et al., 2001) and hac1 (homologous to $\underline{A}$ TF/CRREB $\underline{1}$ ) in yeast (Cox and Walter, 1996; Gonzalez et al., 1999). The spliced HAC1 mRNA (HAC1 ${ }^{i}$ ) is translated into the active Hac1p bZip transcription factor that directly binds to the cis-acting UPR element (UPRE) in promoters of UPR-target genes (Mori et al., 1996). The UPR can be induced by physiological ER stress inducers such as the overexpression of steady misfolded proteins (Oyadomari et al., 2002) or heat stress (Li et al., 2018). Moreover, the UPR can be activated by pharmaceutical ER stress inducers such

Figure 2.6: Sensing of unfolded proteins via the Ire1 pathway. The Ire1 pathway is the most conserved UPR pathway among all eukaryotes. Ire1 is an ER residing kinase/endoribonuclease, which senses unfolded proteins with its luminal domain. Upon ER stress, Ire1 oligomerizes and transautophosphorylates, that activates its RNase domain and the ability to unconventional splice mRNAs. Thus, unconventional splicing of Ire1 is highly specific and solely splicing the mRNA of the UPR transcriptions factor $X B P 1$, encoding for the active transcription factor $\mathrm{XPB} 1^{\mathrm{s}}$ in higher eukaryotes, that activates downstream UPR target genes. $\mathrm{XBP} 1^{\mathrm{s}}$ binds a conserved consensus UPR element (UPRE) in promoters of UPR target genes. However, active Ire1 participates in unspecific mRNA decay (RIDD) of ER-bound mRNA, that supports the UPR by lowering the protein levels entering the ER (modified after (Dillin, 2014)).

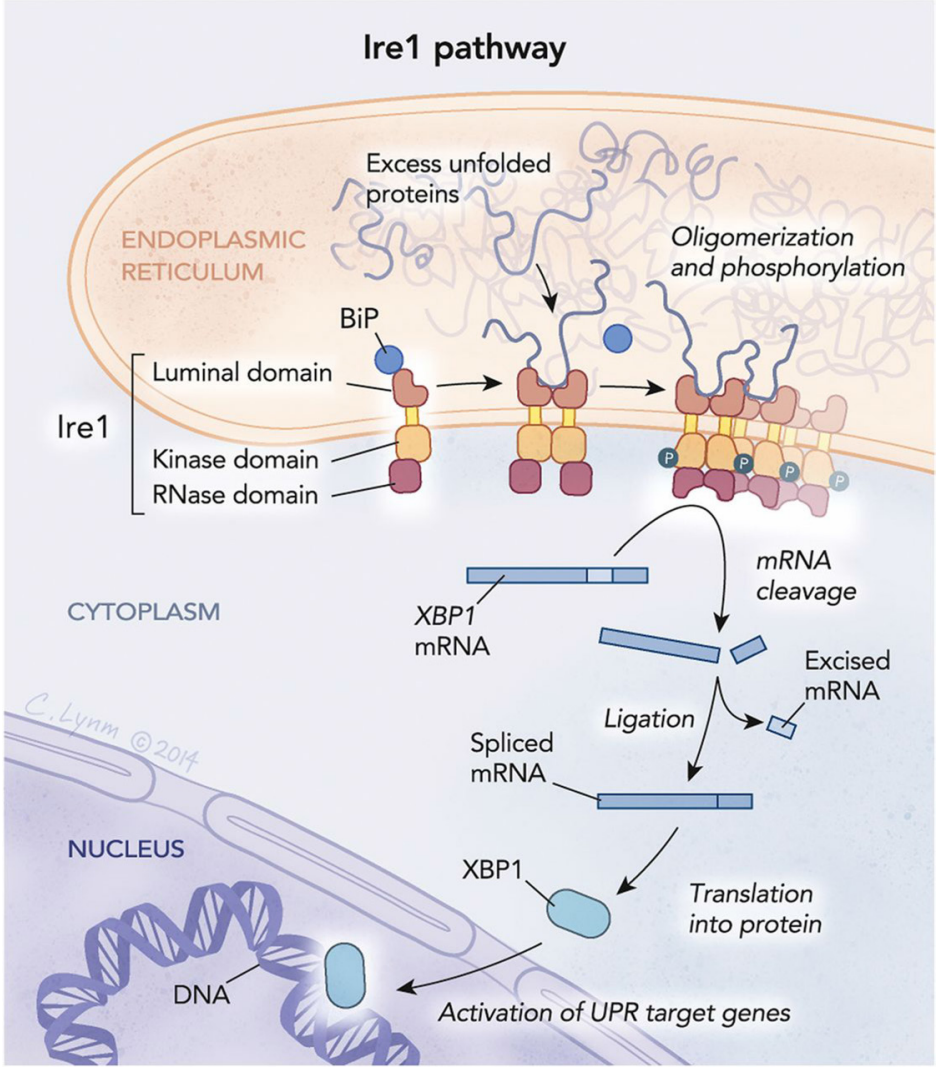


as tunicamycin (TM) or dithiothreitol (DTT). Treatment of cells with tunicamycin inhibits N-linked glycosylation of proteins in the ER, by blocking an initial step of glycoprotein biosynthesis, which leads to accumulation unfolded glycoproteins. In contrast to tunicamycin, the reducing agent dithiothreitol, inhibits the cellular disulfide-bond formation of synthesized proteins, leading to an increase of misfolded proteins in the cytosol (Oslowski and Urano, 2011).

Downstream targets of UPR are involved in regulating cell wall biogenesis, lipid biosynthesis, ERassociated degradation (ERAD) and the formation of an enlarged ER (Reimold et al., 2001; Travers et al., 2000; Sims et al., 2005; Guillemette et al., 2013). In some organisms, IRE1 is also involved in a process called regulated Ire1-dependent decay (RIDD). Here, the active RNase domain of IRE1 unspecifically degrades ER-bound mRNAs under ER stress conditions and by that, indirectly reduces the levels of proteins entering the ER (Hollien et al., 2009).

\subsubsection{The UPR in U. maydis}

The UPR is a conserved virulence determinant in various human and plant pathogenic fungi such as Aspergillus fumigatus (Richie et al., 2009), Cryptococcus neoformans (Cheon et al., 2011), Alternaria brassicicola (Joubert et al., 2011) as well as Ustilago maydis (Heimel et al., 2010a; Heimel et al., 2013). The master regulator of the UPR in U. maydis is Cib1 (lp1 interacting $\underline{\text { bZip }} \underline{1}$ ). Cib1 was initially identified as a Clp1-interacting protein, revealing a direct connection between the UPR and control of pathogenic development (Heimel et al., 2010a). Deletion of cib1 results in a block of pathogenic development after plant infection and increased expression of pathogenesis-related plant genes in maize. Expression of Clp1 alters the UPR and results in a dramatically increased ER stress tolerance. However, in contrast to initial expectations expression levels of cib1 and the UPR target gene bip1, an ER chaperone were reduced by Clp1, suggesting that Clp1 modulation counteracts a hyperactive UPR (Heimel et al., 2013).

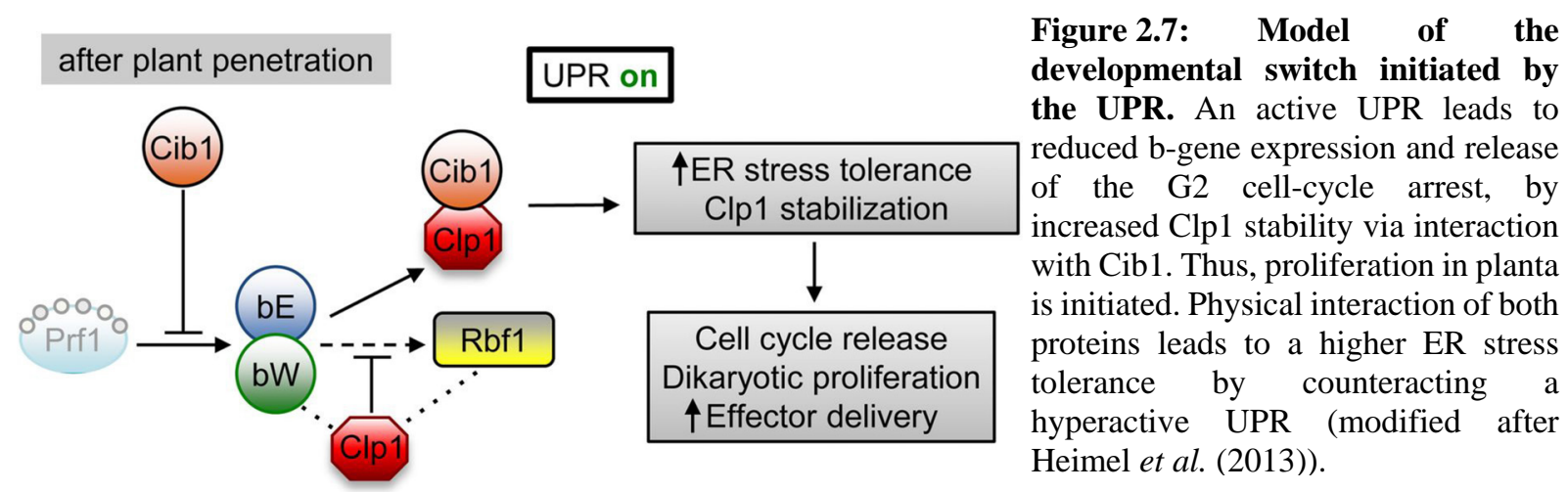

Moreover, the physical interaction between Cib1 and Clp1 leads to increased protein stability of Clp1. In consequence, the Cib1-mediated stabilization promotes accumulation of Clp1, which, in turn, reduces $b$-gene expression through the interaction between Clp1 and bW and Rbf1 (Chapter 2.3). Hence, UPR induction supports the release of the $b$-dependent G2 cell cycle arrest and promotes proliferation in 
planta. Thereby, the interaction between Cib1 and Clp1 might coordinate sexual and pathogenic development with proliferation in planta (Heimel et al., 2013) (Fig 2.7).

Recent studies indicate that the UPR is involved in the effector secretion of $U$. maydis. In general, effectors are secreted proteins by pathogens, which target the hosts to modulate their physiology. This can be achieved by either avoid detection of the pathogen from the host's defense or to suppress the host defense responses (Jonge et al., 2011). In U. maydis, an in silico prediction of UPR elements (UPRE) in promoter regions of 385 predicted effector genes without an enzymatic function, revealed that 76 genes without UPR-related function harboring a UPRE in their promoter. However, only two of the tested genes, tin1-1 and pit2, had an UPR-dependent induction under different ER stress conditions. The effector gene pit2 encoding a cysteine protease inhibitor preventing salicylic acid-induced cell death in planta (Doehlemann et al., 2011). Deletion of the identified UPRE significantly reduced the virulence of deletion mutants. Moreover, protein levels of Pit2 increased upon ER stress and processing of premature Pit2 prior to secretion is a UPR dependent process (Hampel et al., 2016). The effector gene tin11 is part of effector gene cluster 19A and is upregulated during later time points of in planta growth. In contrast to pit2, deletion of a sub-cluster comprising tin1-1 had only a minor effect on virulence (Brefort et al., 2014). Moreover, the ER co-chaperone Dnj1 is upregulated in a cib1-dependent manner and is required for secretion of the effector Cmu1 (chorismate mutase 1) under ER stress conditions (Lo Presti et al., 2016). The UPR in U. maydis is thought to support effector secretion by upregulation of specific effector genes as well as modulation of effector production and modification. This would decrease the load of misfolded or unfolded proteins in the ER during effector secretion, which redirects cellular energy resources to establish a biotrophic interaction with the plant (Hampel et al., 2016). 


\subsection{Signal Peptide Peptidases}

Signal peptide peptidases (SPP) and their close relatives, the signal peptide peptidase-like proteases (SPPLs), are members of the aspartyl intramembrane-cleaving proteases (I-CLiPs). These SPP/SPPLs were initially identified as homologs to presenilin proteases (PSEN) in the human genome. In the past two decades, however, SPP/SPPLs were shown to be present in all studied eukaryotes including fungi, plants, and animals (Ponting et al., 2002; Weihofen et al., 2002; Grigorenko et al., 2002). Interestingly, SPPs have an inverted topology compared to the distantly related PSENs. Therefore, SPP cleavage only occurs in membrane proteins with a type II orientation compared to PSENs, which are only processing type I oriented transmembrane proteins (McLauchlan et al., 2002; Weihofen et al., 2002). SPPs are ER membrane-localized proteins harboring several transmembrane domains (TMD) (Weihofen et al., 2003). Embedded in TMDs are the characteristic YD and GxGD motifs, representing the proteolytic center for intramembrane-cleaving and the QPALLY motif of unknown function (Fig 2.8).

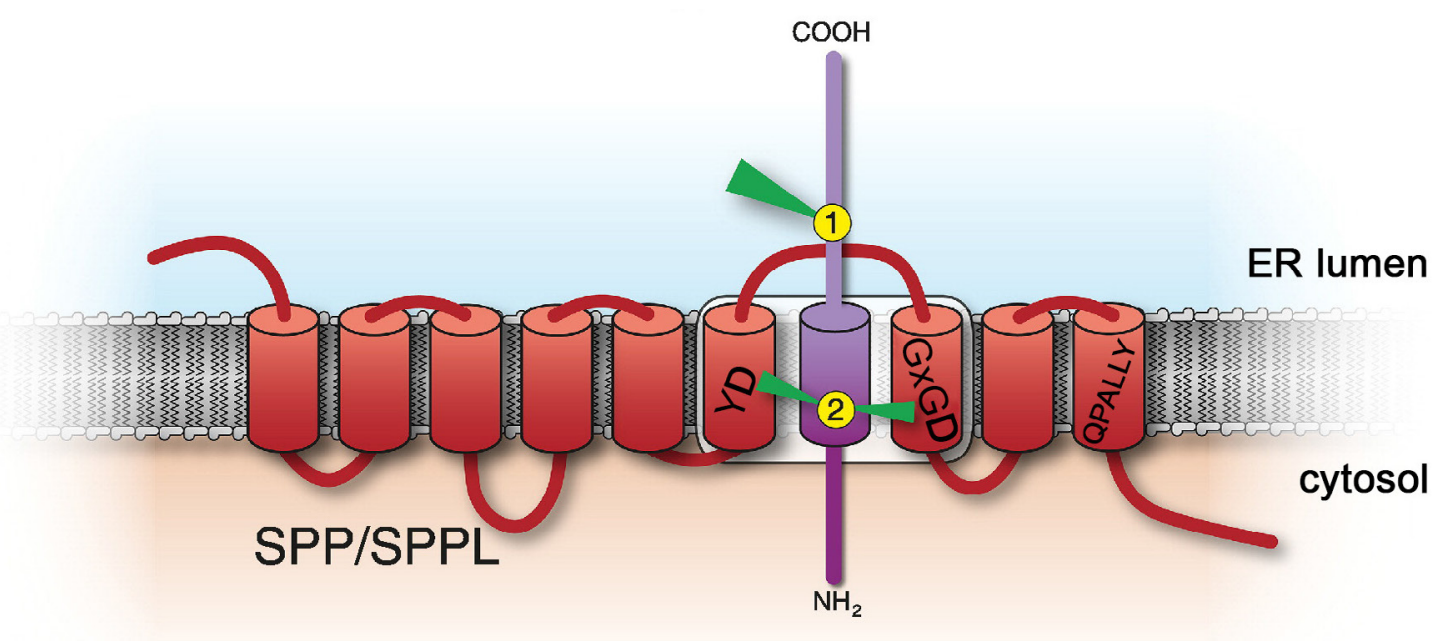

Figure 2.8: Schematic representation of the SPP/SPPL domain topology and substrate processing. SPP and SPPL are multi-pass ER intramembrane proteases cleaving leftover signal peptides in the ER. Substrates of SPP/SPPL require a type II orientation in the ER membrane (C-Terminus in the ER lumen). All SPP/SPPL family members are predicted to consist of 9 TMDs, a conserved YD (TMD6) and GxGD (TMD7) motif within their catalytic center as well as conserved QPALLY motif embedded in TMD9. In general, initial processing of the precursor protein by the signal peptidase (1) is required for the final intramembrane cleavage of the signal peptide (2). Substrate is depicted in purple (modified after (Voss et al., 2013)).

In general, SPPs cleave leftover signal sequences in the ER membrane after processing of the precursor protein by cleavage of a signal peptidase (SP) (Fig 2.8). Thus, SPP cleavage promotes the release of the signal peptide cleavage products and supports the maintenance of the ER homeostasis (McLauchlan et al., 2002; Golde et al., 2009; Lemberg and Martoglio, 2004). In addition, it was shown that SPP is involved in escorting misfolded proteins for degradation via its substrate binding site (Lee et al., 2010). Moreover, in vitro studies of the human SPP showed, that SPP is also involved in the generation of epitopes via signal peptide processing. In contrast to the more prevailing proteasome-dependent pathway of MHC class I epitope production, this pathway represents an alternative route for the generation of these molecules (Lemberg et al., 2001). 
In various organisms, SPPs are involved in pathogenic development. In Aspergillus nidulans and Aspergillus fumigatus, SppA supports fungal pathogenesis by cleavage of SrbA, a decisive factor for hypoxia adaptation (Bat-Ochir et al., 2016). In the malaria parasite Plasmodium falciparum, PfSPP plays a critical role during the intraerythrocytic development of the human pathogen by the preservation of ER homeostasis (Baldwin et al., 2014). Furthermore, SPP was also described in the maturation process of the hepatitis $\mathrm{C}$ virus (HCV), whereby the HCV polyprotein is cleaved within the ER, allowing for the liberation of the core protein, which is necessary to constitute the viron capsid (McLauchlan et al., 2002). By contrast, the role of SPPs during pathogenesis of plant-infecting fungi has not been addressed, yet.

\subsection{Aim of this study}

The UPR is a highly conserved signaling pathway to ensure ER homeostasis under situations of increased demands on the secretory pathway, termed ER stress. Recent studies revealed that the UPR is a critical virulence determinant in various human and plant pathogenic fungi. In particular, a crosstalk between the UPR and pathogenic development was shown in Ustilago maydis. The bZip transcription factor Cib1 is the master regulator of the UPR in U. maydis and was initially identified as an interaction partner of the developmental regulator Clp1. The interaction between Clp1 and Cib1 modulates the UPR output to counteract a hyperactive UPR (Heimel et al., 2013; Heimel et al., 2010a). The aim of this study is, to gain a genome-wide view of the Clp1-dependent modulation of the UPR and to examine how the crosstalk between the UPR and developmental control pathway is accomplished. Furthermore, insight into the transcriptional regulation of an active UPR during Clp1 expression may reveal novel pathogenicity factors in $U$. maydis. 


\section{Results}

In Ustilago maydis, the unfolded protein response is tightly linked to the $b$-dependent signaling cascade that controls pathogenic development. Clp1, a decisive factor for pathogenic development, directly interacts with bW and the master regulator Rbf1, which negatively regulates the $b$-dependent gene expression. This releases the $b$-dependent cell cycle arrest and promotes proliferation in planta. Clp1 is a direct target of the $\mathrm{bE} / \mathrm{bW}$ heterodimer and expression of clp1 mRNA is detectable early after formation of the b-heterodimer. However, the Clp1 protein is detectable only after plant penetration (Heimel et al., 2010a). In addition, Clp1 physically interacts with the central UPR regulator Cib1, by which Clp1 protein stability and ER stress resistance are increased. Plant-specific expression of effector genes results in high demand for protein secretion, leading to ER stress and activation of the UPR. UPR activation is facilitated by Ire1-dependent unconventional splicing of the cib1 mRNA resulting in the active bZIP transcription factor Cib1. The interaction between Clp1 and Cib1 affects the transcriptional output of the UPR and by this prevents deleterious UPR hyperactivation (Heimel et al., 2013).

\subsection{Functional consequences of the interaction between Cib1 and Clp1}

Expression of Clp1 leads to increased ER stress resistance, which is dependent on the physical interaction between Clp1 and Cib1 and thought to protect cells against hyperactivation of the UPR during extended UPR activation in planta (Heimel et al., 2013). U. maydis strains JB1 (WT) and

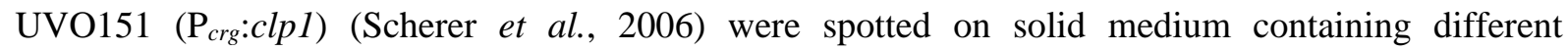
concentrations of the ER stressor tunicamycin (TM), an inhibitor of N-glycosylation, to examine ER

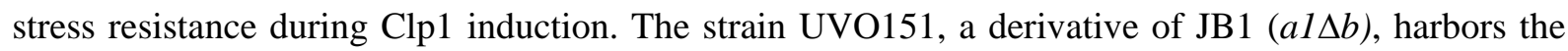
construct $\mathrm{P}_{\text {crg:clp1 }}$ in the ip locus (Chapter 5.4.2). In this strain, clp1 can be expressed with the help of the arabinose-inducible crg1 promoter (Bottin et al., 1996). On solid medium containing low TM concentrations no obvious growth differences between $\mathrm{WT}$ and $\mathrm{P}_{\text {crg }}$ :clp1 strains were apparent. (Fig 3.1, center panel). By contrast, on solid medium containing high TM concentrations, strain UVO151

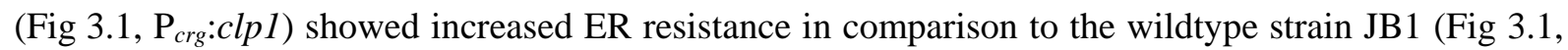
WT, right panel). This indicates that clp1 expression leads to elevated ER stress resistance in U. maydis. 


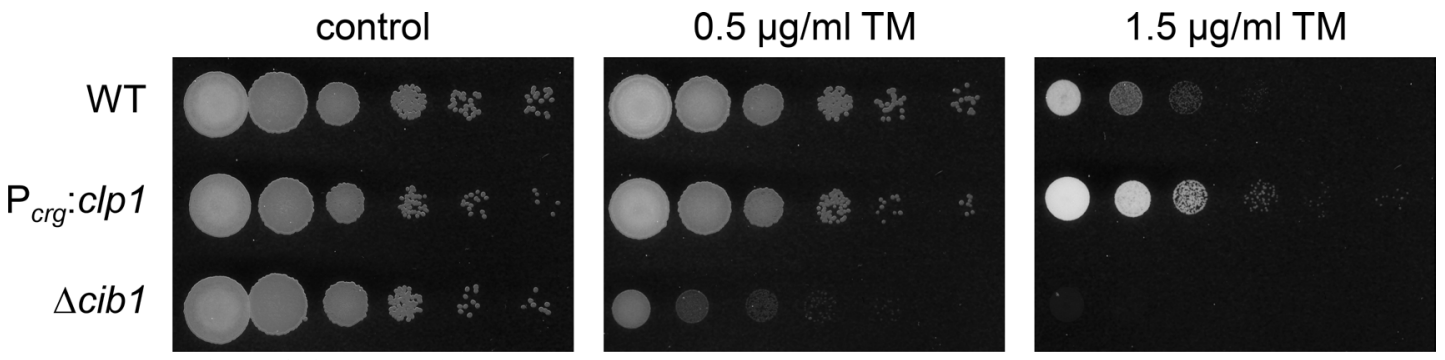

Figure 3.1: Induction of Clp1 during ER stress increases ER stress tolerance. ER stress assay of U. maydis strain JB1 (WT) and derivatives. Strains were grown in YEPS light liquid medium to an $\mathrm{OD}_{600}$ of 1 . Cells were washed once in YNB liquid medium and serial 10-fold dilutions were spotted on YNB solid medium supplemented with $1 \%(\mathrm{w} / \mathrm{v})$ arabinose (YNBA) to induce the $\mathrm{crg} 1$ promoter-driven expression of clp1. Plates were supplemented with TM as indicated to activate the UPR and were incubated $48 \mathrm{~h}$ at $28^{\circ} \mathrm{C}$. $\Delta c i b 1$ served as a positive control for ER stress.

\subsubsection{Fluorescence microscopy of Cib1-GFP strains revealed Clp1- dependent impact on subcellular localization of the fusion protein}

Previous analyses have shown that the bZIP transcription factor Cib1 is localized in the nucleus of U. maydis when the UPR is activated (Heimel et al., 2013). To test the influence of Clp1 expression on the subcellular localization of the Cib1-GFP fusion protein, cells were treated with TM to activate the UPR and subsequently produce the active UPR regulator Cib1-GFP. A clear fluorescence signal was observed in the nucleus after 4 hours of UPR induction. Moreover, cells in which clp1 was induced, showed an additional and strong fluorescence signal in the cytoplasm compared to wildtype strain (Fig 3.2), indicating a Clp1-dependent alteration of subcellular Cib1 localization.

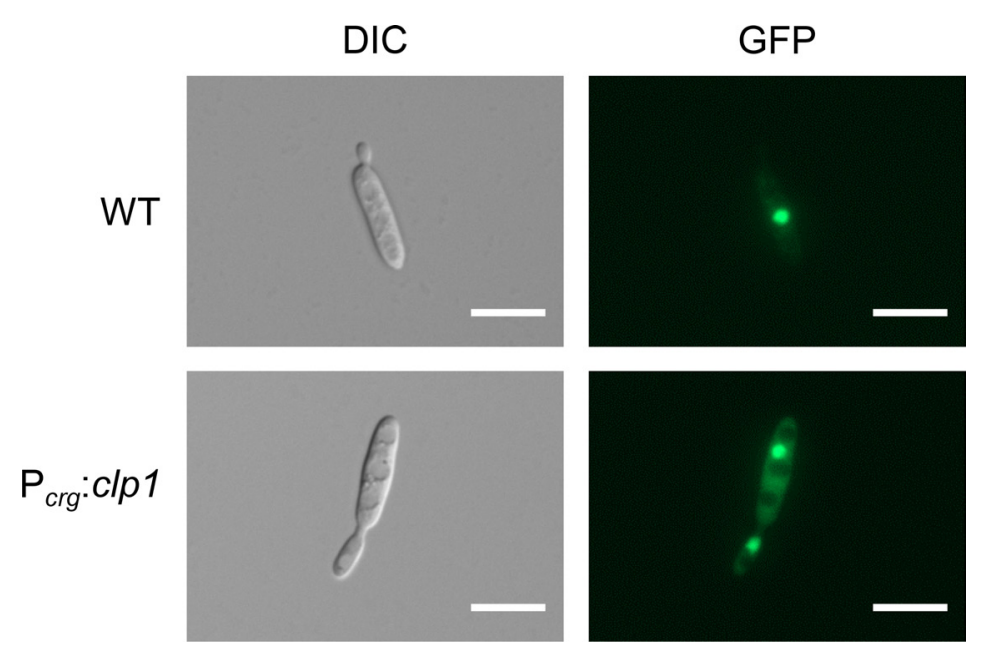

Figure 3.2: Cib1-GFP localization is altered upon Clp1 induction. The strains JB1cib1-GFP (WT) and UVO151cib1-GFP ( $\mathrm{P}_{\text {crg: }}$ :clp1) were grown in liquid complete medium (CM) supplemented with $1 \%$ (w/v) glucose (CMG) to an $\mathrm{OD}_{600}$ of 0.8 to 1 . Cells were subsequently shifted to CM medium supplemented with $1 \%$ (w/v) arabinose (CMA) to induce the $c r g 1$ promoter-driven gene expression of $c l p 1$ and were treated $4 \mathrm{~h}$ at $28^{\circ} \mathrm{C}$ with TM (5 $\mu \mathrm{g} / \mathrm{ml}$ f.c.) to activate the UPR. Strains expressing Clp1 upon ER stress showed an additional and strong cytoplasmic GFP signal. DIC = differential interference contrast. Exposure time of GFP channel was set to $500 \mathrm{~ms}$. Scale bar $=10 \mu \mathrm{m}$. 


\subsubsection{Induced expression of Clp1 leads to increased abundance of Cib1 protein and decreased $\operatorname{cib} 1^{s}$ transcript levels}

The interaction between Clp1 and Cib1 results in an increased ER stress resistance (Heimel et al., 2013;

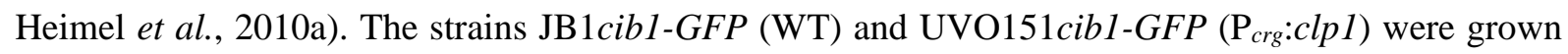
in $\mathrm{CMG}$ to an $\mathrm{OD}_{600}$ of 0.35 , to examine the impact of Clp1 expression on Cib1 protein and cib1 ${ }^{s}$ transcript levels. Subsequently, cells were shifted to CMA to induce clp1 expression and were treated 4

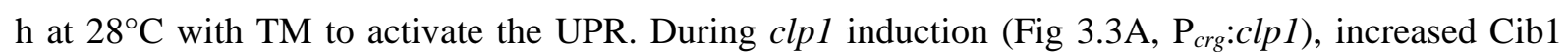
protein levels were observed in comparison to the wildtype. Moreover, a higher migrating band is visible in the wildtype strain, suggesting a post-translational modification of the protein (Fig 3.3A, WT). In contrast to the increased protein levels of Cib1-GFP, $c i b 1^{s}$ transcript levels were significantly lower during Clp1 expression (Fig 3.3B, $\mathrm{P}_{\text {crg: }}$ :clp1, P-value $\leq$ 0.001) compared to the wildtype strain (Fig 3.3B, WT). This indicates that a post-transcriptional mechanism controls the abundance of Cib1 in a Clp1dependent manner. Thus, the increased protein levels of Cib1 and the decreased $c i b 1^{s}$ expression raise the question, whether an enhanced translation of the cib $1^{s}$ mRNA or increased Cib1 protein stability leads to this result.

A

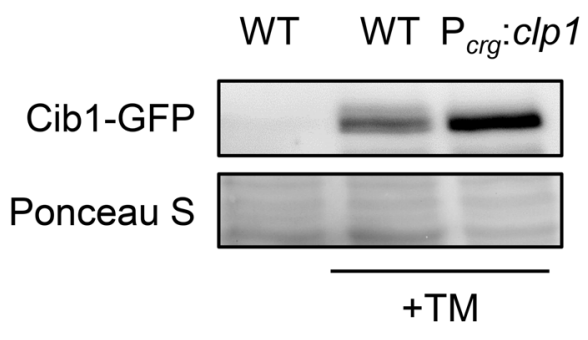

B

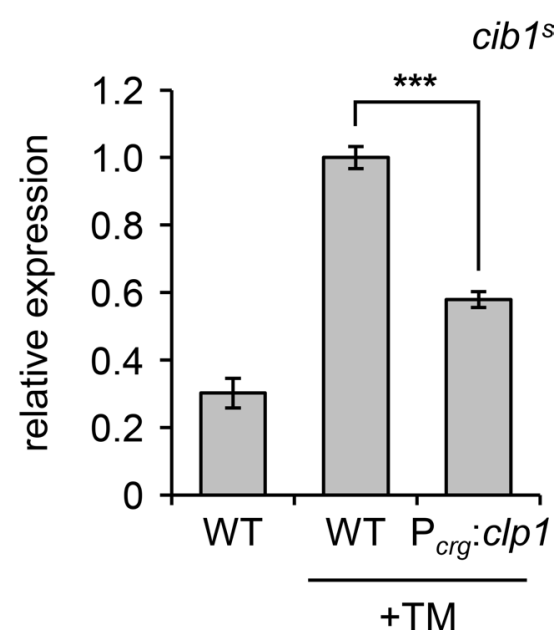

Figure 3.3: Western hybridization of Cib1-GFP and qRT-PCR of $c i b 1^{s}$ in dependency of Clp1 expression.

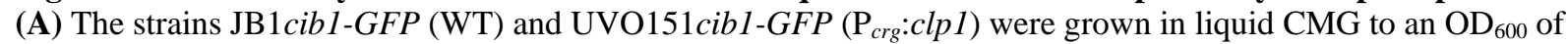
0.35 and subsequently shifted in CMA to induce the $\mathrm{crg} 1$ promoter-driven gene expression of $c l p 1$. Cells were treated $4 \mathrm{~h}$ at $28^{\circ} \mathrm{C}$ with TM (5 $\mu \mathrm{g} / \mathrm{ml}$ f.c.) for UPR activation. Samples were resolved by SDS-PAGE (10\%) and analyzed by Western hybridization. For detection of the Cib1-GFP fusion protein, a GFP specific antibody was used. Cib1-GFP levels are increased in strains expressing clp1 in comparison to the WT control. Ponceau S stained bands were used as a loading control. (B) Strains and growth condition were the same as described in (A). cib1 ${ }^{\text {s }}$ transcript levels were analyzed by qRT-PCR. Expression of $c i b 1^{s}$ is significantly decreased (P-value $\leq 0.001$ ) in strains expressing Clp1. eIF2b (UMAG_04869) was used for normalization. The experiment was performed in three biological replicates with two technical replicates, each. Error bars represent the standard deviation of the mean of the biological replicates (SD). 


\subsubsection{Clp1 expression increases Cib1 protein stability}

Physical interaction between Cib1 and Clp1 leads to accumulation and increased stability of Clp1 (Heimel et al., 2013). A cycloheximide chase assay (CHX), as well as a doxycycline (DOX)-based promoter shut-off assay (Zarnack et al., 2006) were performed, to examine the influence of clp1 expression on Cib1 stability. The strains JB1cib1-GFP and UVO151cib1-GFP in the CHX experiment,

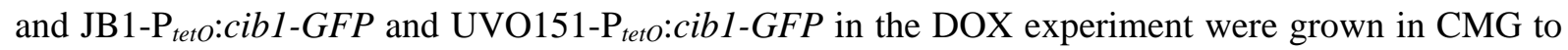
an $\mathrm{OD}_{600}$ of 0.35 . Subsequently, cells were shifted to CMA to induce $c l p 1$ expression and were treated $4 \mathrm{~h}$ at $28^{\circ} \mathrm{C}$ with TM to activate the UPR. After 4 hours of clp1 induction and UPR activation, cycloheximide or doxycycline was added to the culture to inhibit protein synthesis or stop expression of cib1 mRNA, respectively. Cib1-GFP levels were monitored over time and were quantified relative to T0 (Fig 3.4A and 3.4B). In the cycloheximide chase assay, protein stability of Cib1 was significantly increased $(\mathrm{P} \leq 0.05)$ after 60 minutes (T60) of $c l p 1$ induction compared to WT control (Fig 3.4A). This observation was as well confirmed in the promoter shut-off assay with DOX. A significant increase of Cib1-GFP protein stability in the $\mathrm{P}_{\text {crg:clp1 }}$ strain was observed 1 hour $(\mathrm{T} 1, \mathrm{P} \leq 0.05), 2$ hours (T2, $\mathrm{P} \leq$ 0.01), 3 hours (T3, P $\leq 0.01)$ and 4 hours (T4, P $\leq 0.05)$ after DOX treatment compared to the WT (Fig 3.4B). Both results demonstrate that Cib1-GFP is stabilized upon Clp1 expression. 
A
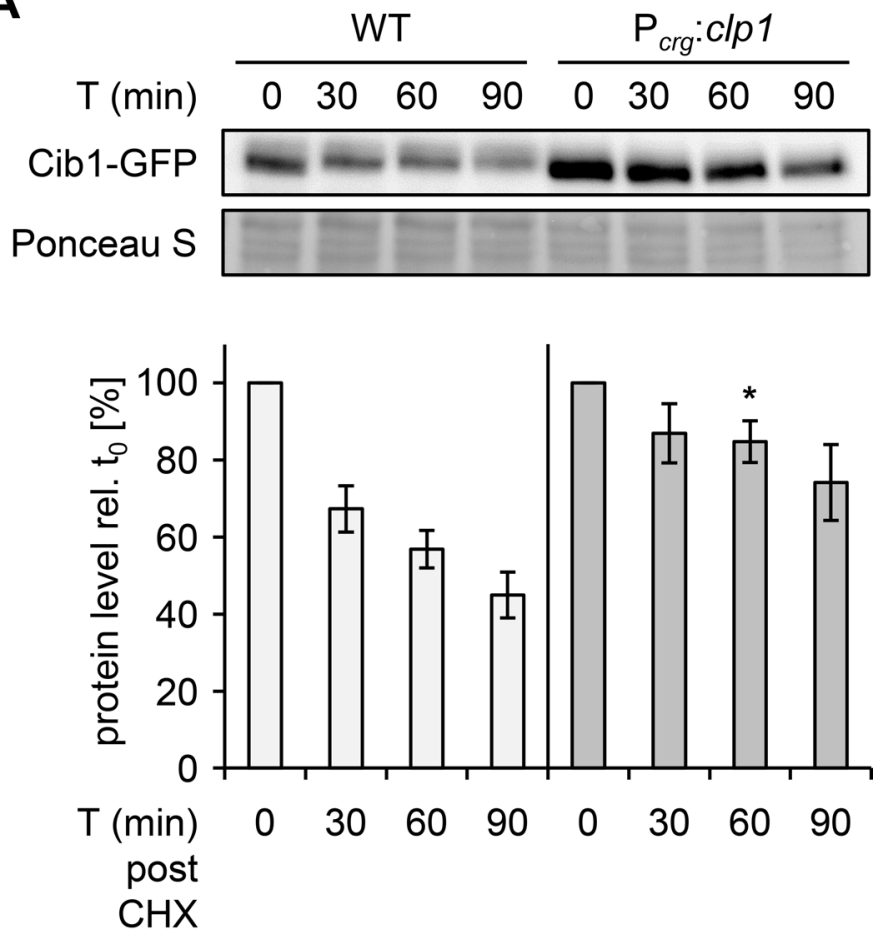

B
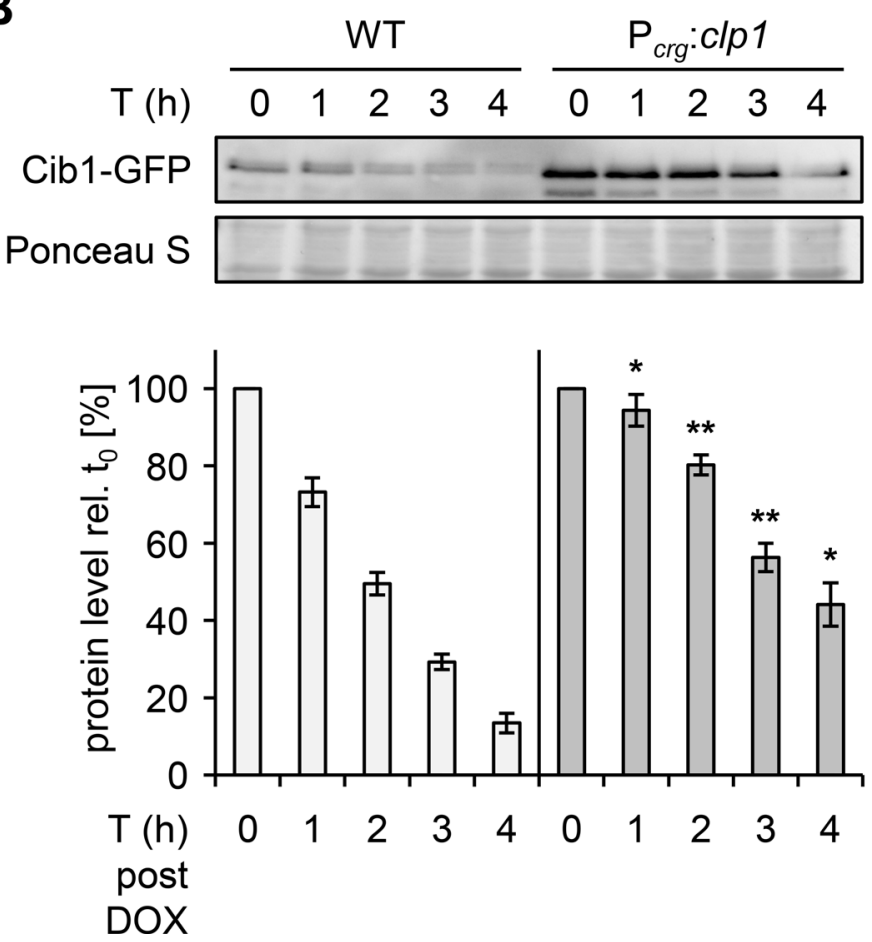

Figure 3.4: Clp1 expression increases

Cib1-GFP protein levels. (A) For the cycloheximide chase assay, the strains JB1cib1GFP and UVO151cib1-GFP were exponentially grown in CMG and shifted to CMA liquid medium to induce clp1 expression and were treated with $\mathrm{TM}(5 \mu \mathrm{g} / \mathrm{ml}$ f.c.) to activate the UPR for $4 \mathrm{~h}$ at $28^{\circ} \mathrm{C}$. After $4 \mathrm{~h}$, $100 \mu \mathrm{g} / \mathrm{ml}$ CHX was added to inhibit protein synthesis. Samples were taken before (T0), and after $30 \mathrm{~min}$ (T30), $60 \mathrm{~min}$ (T60) and $90 \mathrm{~min}$ (T90) of CHX treatment. Protein extracts were separated by SDS-PAGE (8\%) and analyzed by Western hybridization with a GFP specific antibody. Ponceau S stained membranes served as a loading control and were used for normalization of protein levels. ImageJ was used for calculation of protein levels relative to T0. Depicted values represent the mean of three biological replicates. Error Bars represent the standard error of the mean (SEM). Statistical significance was calculated using Student's $t$ test. *P-value $\leq 0.05$. (B) For the doxycyclinebased promoter shut-off assay the strains JB1$\mathrm{P}_{\text {teto }}: c i b 1-G F P$ and UVO151-P $\mathrm{P}_{\text {teto }}: c i b 1-G F P$ were grown as described in (A). After $4 \mathrm{~h} \mathrm{UPR}$ induction and clp1 expression, $10 \mu \mathrm{g} / \mathrm{ml}$ DOX was added to shut-off gene expression of cib1GFP. Samples were taken before (T0), and after $1 \mathrm{~h}$ (T1), $2 \mathrm{~h}$ (T2), $3 \mathrm{~h}$ (T3) and $4 \mathrm{~h}$ (T4) of DOX treatment. Protein extracts were separated by SDS-PAGE (8\%) and analyzed by Western hybridization with a GFP specific antibody. Ponceau $\mathrm{S}$ stained membranes served as a loading control and were used for normalization of protein levels. ImageJ was used for calculation of protein levels relative to T0. Depicted values represent the mean of three biological replicates. Error Bars represent the SEM. Statistical significance was calculated using Student's $t$-test. ${ }^{*}$ P-value $\leq 0.05$ and ${ }^{* *} \mathrm{P}$ value $\leq 0.01$. 


\subsubsection{Cib1 is a phosphoprotein and phosphorylation is reduced in strains expressing Clp1}

In western hybridization experiments detecting Cib1-GFP, a higher migrating protein band was specifically detected in the wildtype but not the $c l p 1$ expressing strain (Figure 3.3A, 3.4A and 3.4B, WT). This observation might be the result of post-translational modification of the protein. A $\lambda$ phosphatase assay was performed, to test whether Cib1 is modified by phosphorylation. Strains JB1cib-

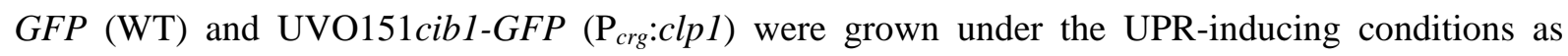
described in chapter 3.1.2. The Cib1-GFP fusion protein was pulled down with magnetic agarose GFPtrap beads and was treated with $\lambda$-phosphatase while Cib1-GFP was still bound to the beads. Phosphatase inhibitor was added to inhibit the $\lambda$-phosphatase as indicated. In samples obtained from the WT strain, a higher migrating band is observable compared to samples from the $\mathrm{P}_{\text {crg:clp}}$ 1 strain without $\lambda$ phosphatase treatment (Fig 3.5, lane 1 vs. lane 4). However, the blurred and higher migrating protein band in the WT focalized to a distinct lower migrating protein band after addition of $\lambda$-phosphatase (Fig 3.5 , lane 1 vs. lane 2), which is similar to the untreated conditions once the phosphatase inhibitor was added (Fig 3.5, lane 1 vs. lane 3). In contrast to this observation, almost no change of protein band migration can be observed between conditions with clp1 expression. Moreover, the protein bands are located on a similar height as in the treated wildtype strain with the inhibited phosphatase (Fig 3.5, lane 2 vs. lane 4, 5 and 6). This indicates that the clp1-dependent protein band migration of Cib1 is caused by phosphorylation of the protein.

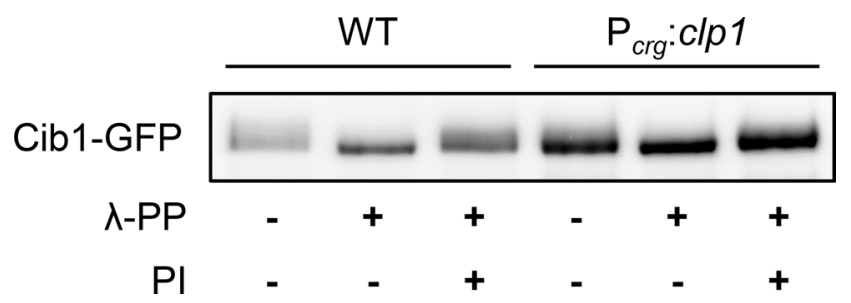

Figure 3.5: Altered phosphorylation of Cib1-GFP by expression of clp1. Western hybridization of Cib1-GFP after $\lambda$-phosphatase treatment. Protein extracts were prepared from the strains JB1cib1-GFP (WT) and UVO151cib1-GFP ( $\mathrm{P}_{\text {crg: }}$ :lp1). Growth conditions and treatment with TM were identical as described in chapter 3.1.1. The Cib1-GFP fusion protein was pulled down using magnetic agarose (MA) GFP-trap beads (Chromotek) and phosphatase treatment with $\lambda$-phosphatase ( $\lambda$-PP, NEB) was performed on beads. Phosphatase inhibitor (PI) was used to inhibit $\lambda$-phosphatase function. For detection of the Cib1-GFP fusion protein, a GFP specific antibody was used. The assay was performed in three biological replicates. 


\subsubsection{LC-MS analysis confirms Cib1 phosphorylation and reveals altered phosphorylation patterns upon Clp1 expression}

A NetPhos-3.1 (Blom et al., 1999) analysis of the Cib1 protein sequence was performed to predict potential serine, threonine or tyrosine phosphorylation sites (Fig 3.6B). NetPhos predicted 68 serines, 18 threonines and 3 tyrosines as potential phosphorylation sites in the Cib1 protein with a threshold value of 0.5 (scale from 0 to 1 ) (Figure 3.6B, gray line). Moreover, a liquid chromatography mass spectrometry (LC-MS) assay was performed, to examine potential phosphorylation sites of Cib1 in presence and absence of Clp1. Strains JB1 cib1-GFP (WT) and UVO151 cib1-GFP ( $\mathrm{P}_{\text {crg:clp1) were }}$ grown as described in chapter 3.1.2. GFP-trap enrichment of Cib1-GFP was performed from cell lysates of both strains. Immunoprecipitated Cib1-GFP was resolved on an SDS-PAGE and an excised, Coomassie-stained Cib1-GFP band (Appendix Fig 7.5) was used for in-gel trypsin digestion. Subsequently, eluted peptide samples were submitted to LC-MS analysis for phosphopeptide identification with the targeted selected ion monitoring (tSIM) method.

Table 3.1: Identified phosphosites of Cib1-GFP by LC-MS analysis. Ratios of the peptide spectrum match (PSM) values between $\mathrm{P}_{\text {crg }}$ :clp1 and WT were calculated for each identified phosphopeptide.

\begin{tabular}{|c|c|c|c|c|}
\hline \multirow[b]{3}{*}{ position } & \multirow[b]{3}{*}{ Sequence } & \multicolumn{3}{|c|}{ P crg:clp1 vs. WT } \\
\hline & & & $(n=3)$ & SD \\
\hline & & phosphosite & [ratio] & {$[+l-]$} \\
\hline $2-31$ & TSTTTSTPPMFAVAQASTPSSPSAFASSSR & Ser22 & 1.40 & 0.44 \\
\hline $32-55$ & LSETPVKQETHHIALADACSNSSK & Ser33 & 0.90 & 0.25 \\
\hline $369-401$ & TAAQDQGAPTSATPSEPVSVGEGYAAAAGNALR & Thr381 & 0.73 & 0.12 \\
\hline $462-475$ & RFQLLTSPLLATER & Ser468 & 0.67 & 0.10 \\
\hline
\end{tabular}

In this approach, four different phosphopeptides could be identified (Table 3.1), which were present in

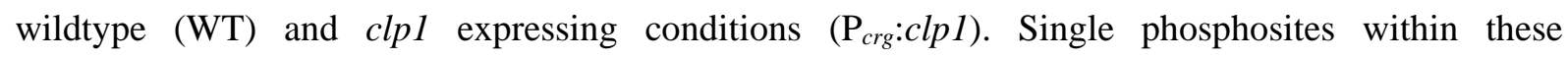
phosphopeptides are highlighted in red (Table 3.1). Two out of four identified phosphopeptides are derived from the N-terminal region of the protein (Table 3.1 and Fig 3.6A, Ser22 and Ser33), and two from the C-terminal region of the Cib1 (Table 3.1 and Fig 3.6A, Thr381 and Ser 468). The Ser468 phosphosite is located in the previously (Heimel et al., 2013) identified Clp1 interaction domain (Fig 3.6A, Clp1-ID) and is predicted by the NetPhos analysis to be phosphorylated by a p38 mitogenactivated protein kinase (prediction score (ps): 0.572). For the phosphosites Ser22 (ps: 0.984), Ser33 (ps: 0.994) and Thr381 (ps: 0.931) no specific kinase was predicted by NetPhos (Appendix Table 1). 
A

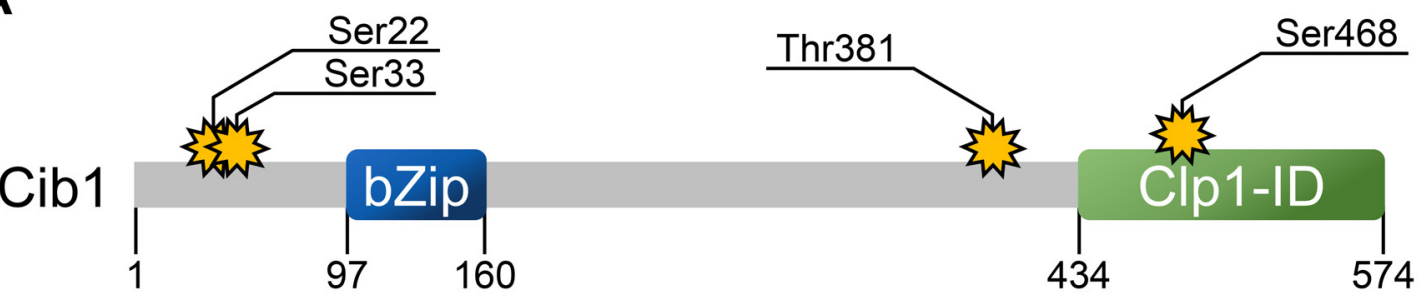

B

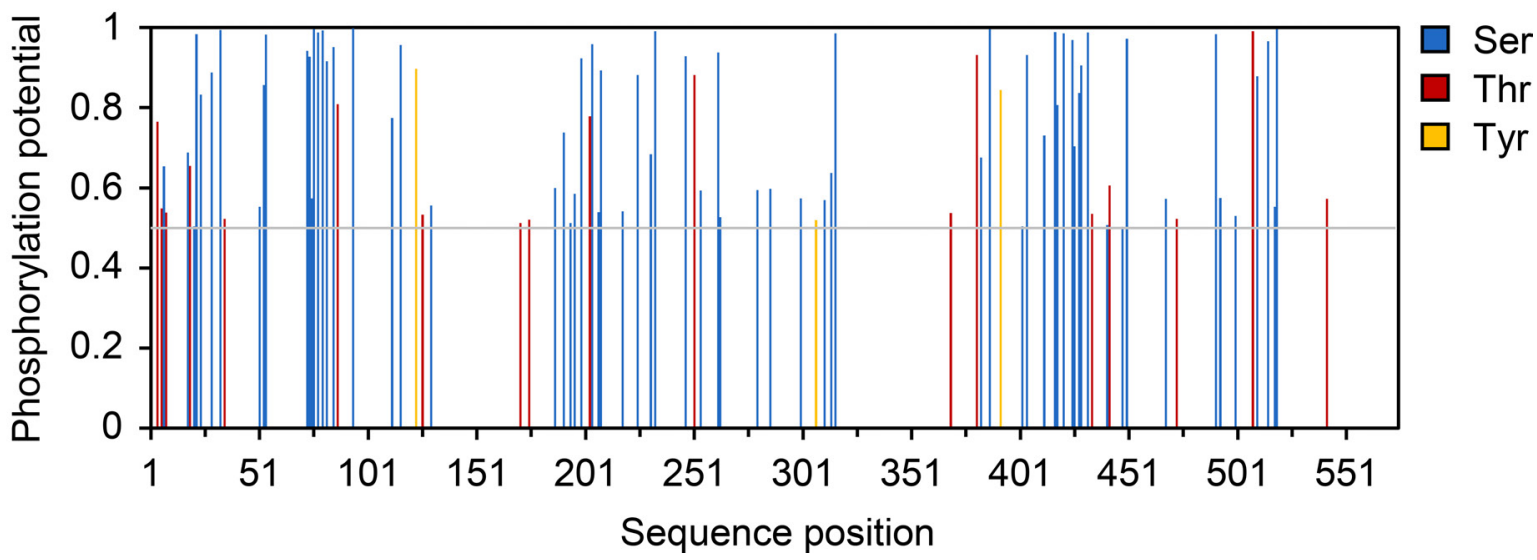

Figure 3.6: Schematic overview of Cib1 domains and putative phosphosites. (A) Schematic representation of Cib1 domain structure. The protein consists of the basic leucine zipper domain (bZip, blue box, 63AA) and the recently identified (Heimel et al., 2013) Clp1 interaction domain (Clp1-ID, green box, 140AA). Identified phosphosites by LC-MS analysis (Table 3.1) are depicted as yellow stars. The total length of the protein is 574AA. (B) Potential phosphorylation sites identified by the NetPhos-3.1 analysis (Blom et al., 1999). Y-axis is representing the prediction score of possible phosphorylation, whereas the $\mathrm{x}$-axis is representing the sequence position within the Cib1 sequence. Phospho-serines, phospho-threonines and phospho-tyrosines are depicted in blue, red and yellow bars, respectively. The threshold of the prediction score for positive phosphosites prediction was 0.5 (gray line).

In addition, the usage of the label-free quantification (LFQ) method for LC-MS analysis is an alternative approach for comparative quantification of proteins (Patel et al., 2009). To only scan masses of specified phosphopeptides, the measurement was performed in the tSIM mode. This enables a quantitative comparison of Cib1-GFP phosphopeptides between wildtype (WT) and clp1 expressing ( $\mathrm{P}_{\text {crg:clp1) }}$ conditions.

Ratios of the PSM values between both strains and of each identified phosphopeptide were calculated (Table 3.1). Therefore, values above 1 representing a higher PSM count in wildtype (WT) compared to

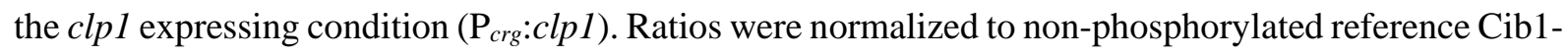
derived peptides in both samples. Among the identified phosphosites depicted in Table 3.1, Ser22 (ratio $1.40+/$ - 0.44) was higher phosphorylated and Thr381 (ratio $0.73+/$ - 0.12) as well as Ser468 (ratio 0.67 +/- 0.10) were less phosphorylated upon clp1 induction. Phosphorylation of Ser33 (ratio 0.90 +/- 0.25) was similar between wildtype and clp1 expressing conditions. Since Thr381 and Ser468 were less phosphorylated upon Clp1 induction and both phosphosites are located near or in the Clp1 interaction domain of Cib1, respectively, this may indicate that Clp1 has a protective effect on the phosphosites during interaction with Cib1. 
To examine, if the newly identified phosphorylation sites Thr381 and Ser468 of Cib1 are involved in pathogenicity of $U$. maydis, phosphomimetic and phospho-null mutations in the solopathogenic haploid strain SG200 were generated. By site-directed mutagenesis the cib1 coding sequence was altered to replace amino acid residues Thr381 and Ser468 by alanine (T381A or S468A) and prevent phosphorylation at these sites, generating strains SG200cib1 $1^{\text {T381A }}$-GFP and SG200cib1 ${ }^{\text {S468A }}$-GFP. To mimic phosphorylation, site-directed mutagenesis of the cib1 coding sequence was used to replace amino acid residues Thr381 and S468 with glutamic acid (T381E or S468E), to generate the strains SG200cib1 $1^{\text {T381E }}$-GFP and SG200cib1 ${ }^{\text {S468E }}$-GFP. In addition, double mutants were generated in which both phosphomimic or phospho-null mutations were combined, generating the strains

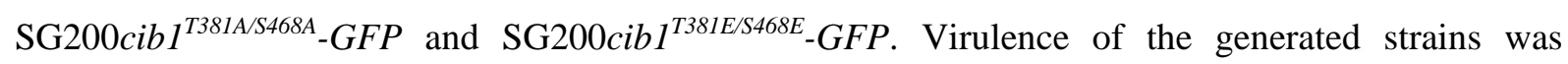
examined in a plant infection assay. Strains were inoculated into 7-day-old maize seedlings and disease symptoms were rated 8 days after inoculation (dpi). However, the mutant strains were not impaired in virulence compared to wildtype (Fig 3.7). This indicates that the identified phosphosites Thr381A and Ser468A are not involved in pathogenicity.

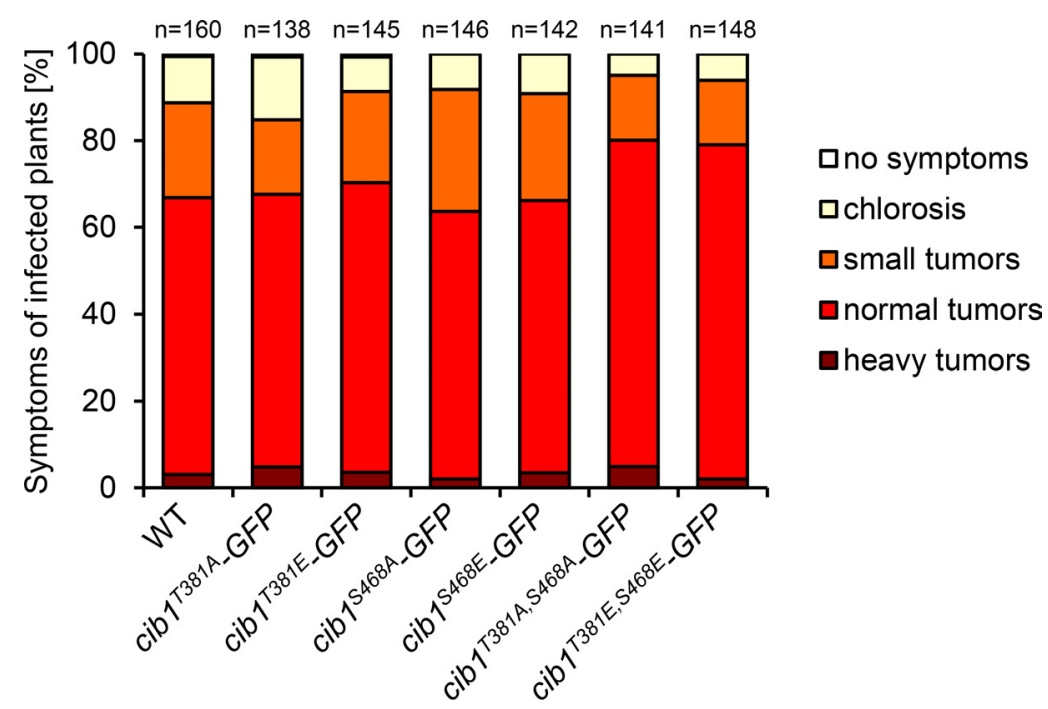

Figure 3.7: Phosphomutations of Cib1 had no impact on pathogenicity. U. maydis strain SG200 (WT) and derivatives were inoculated into 7-day-old maize seedlings. Disease symptoms were rated $8 \mathrm{~d}$ after inoculation and grouped into categories depicted on the right side. $\mathrm{n}$ represents the number of inoculated plants in a single infection experiment. No major change in virulence could be observed.

\subsection{Consequences of Clp1 expression on UPR gene regulation}

The impact of clp1 expression on UPR gene regulation was previously investigated for a subset of known UPR target genes (Heimel et al., 2013). To identify a set of Cib1 regulated UPR core genes and to study the transcriptional changes by Clp1 on UPR target gene expression on a genome-wide level, a transcriptome analysis (RNAseq) was performed. To identify alterations of the DNA binding specificity of Cib1 upon clp1 induction, a comparative chromatin immunoprecipitation sequencing assay (ChIPseq) was performed. The ChIPseq analysis revealed novel UPR elements (UPRE) in cib1 regulated genes of U. maydis. 


\subsubsection{RNAseq analysis identifies a set of UPR core genes}

To identify genes upregulated during UPR stress, an RNAseq analysis was performed with RNA extracted from strains grown under ER stress induction. Strains JB1 (WT), JB1 $\Delta$ cib1 ( $\Delta$ cib1) and

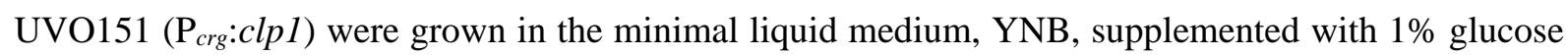
$(\mathrm{w} / \mathrm{v})$ to an $\mathrm{OD}_{600}$ to 0.25 and subsequently shifted to YNB liquid medium containing $1 \%$ arabinose $(\mathrm{w} / \mathrm{v})$ to activate the $\operatorname{crg} 1$ driven gene expression. Cells were treated with $\mathrm{TM}$ for 4 hours at $28^{\circ} \mathrm{C}$. Prepared RNA was submitted for library construction and subsequent sequencing in the single-end mode. Raw RNAseq reads were aligned using the STAR software (Dobin et al., 2013). Reads per million (RPM) were calculated and differential gene expression was analyzed with DESeq2 (Love et al., 2014) using an FDR threshold of 0.05 and a $\log 2$ fold change $(\log 2 \mathrm{FC}$ ) threshold of 2 (list with $\log 2 \mathrm{FC} \geq 1$ in Appendix Table 2).

For identification of UPR core genes, log2 fold-changes of gene expression of the strains WT, WT +TM and $\Delta c i b 1+\mathrm{TM}$ were compared with each other. Comparison between WT and WT + TM should reveal genes (if $\log 2 \mathrm{FC} \geq-/+2$ ), which are differentially regulated upon ER stress. In contrast, the comparison between WT +TM and $\Delta c i b 1+\mathrm{TM}$ should reveal genes (if $\log 2 \mathrm{FC} \geq-/+2$ ), which are differentially regulated in the WT strain upon ER stress induction and exclude genes, which are unspecifically regulated by TM induced ER stress in the $\Delta c i b 1$ strain. The first comparison (Fig 3.8, WT vs. WT +TM, blue circle) revealed that of 204 differentially expressed genes, 103 genes were upregulated, whereas 101 genes were downregulated upon ER stress. The second comparison (Fig 3.8, WT +TM vs. $\Delta c i b 1$ +TM, yellow circle) revealed that of 381 differentially expressed genes, 217 genes were upregulated and 164 genes were downregulated during UPR activation. Both sets were used to generate an intersection (Fig 3.8, overlap), which shared a total of 115 differentially regulated genes. 65 of these genes were upregulated, whereas 50 genes were downregulated during UPR activation (Fig 3.8, overlap).

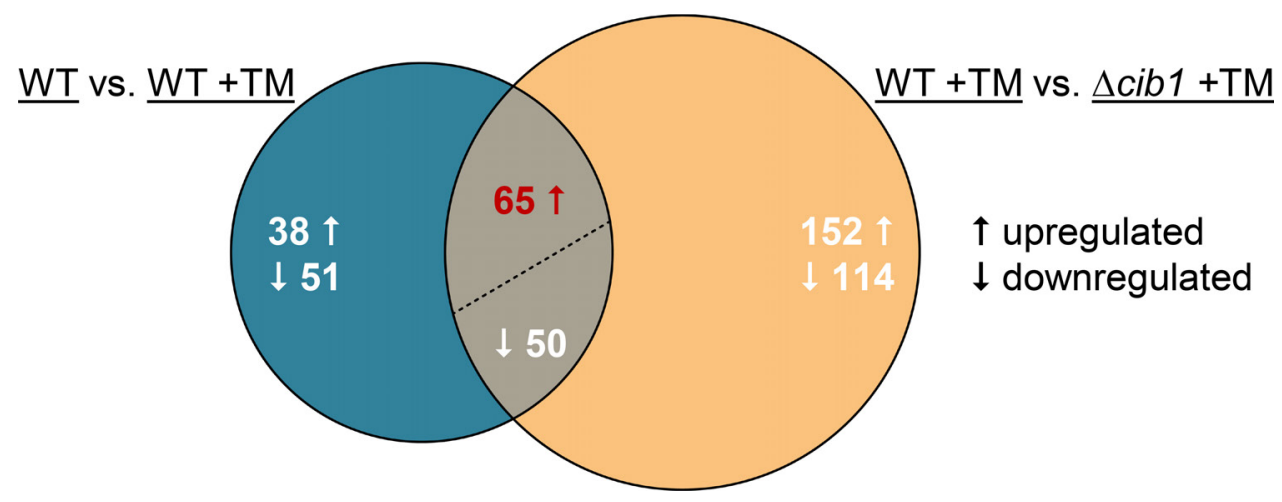

Figure 3.8: RNAseq analysis identified UPR core genes in $\boldsymbol{U}$. maydis. Venn diagram shows the intersection of differentially regulated genes of WT compared with WT+TM (blue circle) and WT +TM compared with WT $\Delta$ cib1 $+\mathrm{TM}$ (yellow circle). Depicted numbers represent differentially regulated genes $(\log 2 \mathrm{FC} \geq-/+2)$. Both sets share 115 genes (overlap), of which 65 genes were upregulated (red text) and 50 downregulated during UPR activation. Upward and downward arrows indicate upregulation and downregulation, respectively. A complete list of filtered UPR core genes $(\log 2 \mathrm{FC} \geq-/+2$ and $\geq-/+1)$ can be found in Appendix Table 2 . 
The 65 identified upregulated UPR core genes were further analyzed using the functional catalog of the MIPS database (http://mips.helmholtz-muenchen.de/funcatDB) to analyze enrichment of the genes in specific functional categories. Genes are enriched in the functional categories "protein fate (folding, modification, destination)" (P-value $\leq$ 0.001, 22 genes), "protein binding" (P-value $\leq$ 0.05, 21 genes), "cellular transport, transport facilities and transport routes" (P-value $\leq 0.01,20$ genes) and "unfolded protein response (e.g. ER quality control)" (P-value $\leq 0.001,8$ genes). The complete FunCat analysis can be found in Appendix Table 4. Taken together, the 65 UPR core genes are upregulated during ER stress in strains with a functional cib1 expression (Fig 3.8) and enriched in functional categories with an ER and UPR-related function (Fig 3.9). This might indicate that the UPR core genes contribute to restoring the ER homeostasis and/or are involved in pathogenicity of U. maydis as virulence factors. Therefore, the identified set of UPR core genes were subjected to further analyses.

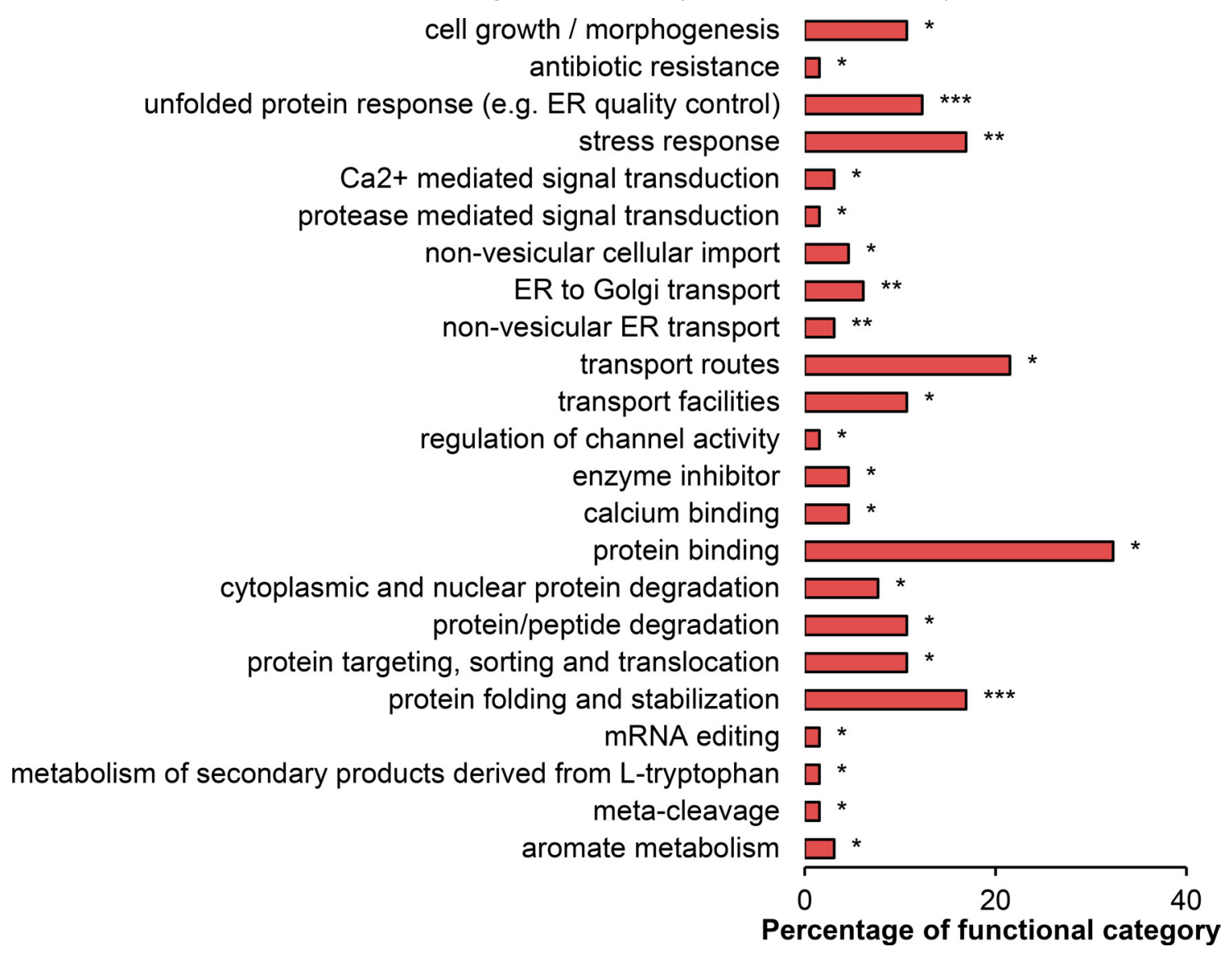

Figure 3.9: UPR core genes are enriched in functional categories with ER and UPR-related function. To categorize the 65 identified UPR core genes, the functional catalog (FunCat) by the MIPS database (http://mips.helmholtz-muenchen.de/funcatDB) was used. Bars are representing the percentage of genes occurring in the functional class. Calculated P-values by FunCat are depicted as asterisks ( ${ }^{*} \mathrm{P}$-value $\leq 0.05,{ }^{* * \mathrm{P}}$-value $\leq 0.01$ and $* * * \mathrm{P}$-value $\leq 0.001)$. Genes can occur in more than one category. A complete list of functional categories can be found in Appendix Table 4. 


\subsubsection{RNAseq reveals modulation of UPR core genes by Clp1}

In recent studies, a clp1-dependent UPR modulation was observed for a small set of UPR marker genes (Heimel et al., 2013). To address the clp1-dependent modulation of UPR core genes identified in this study, expression of UPR core genes was compared between WT and the clp1 expressing strain

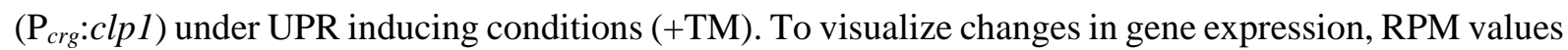
were used to generate a hierarchically clustered heat map (Fig 3.10). RNAseq analysis was performed on strains grown in axenic culture under ER stress induction. In addition, in planta expression of all UPR core genes was depicted in Figure 3.10 (2, 4 and 6 dpi), which was published recently by Lanver et al. (2018). By comparison, 47 of the 65 UPR core genes identified in the present study were found to be upregulated in planta, 13 are not regulated and 5 are downregulated at 2dpi. At 4 dpi, 12 UPR core genes are not regulated and 6 are downregulated in planta.

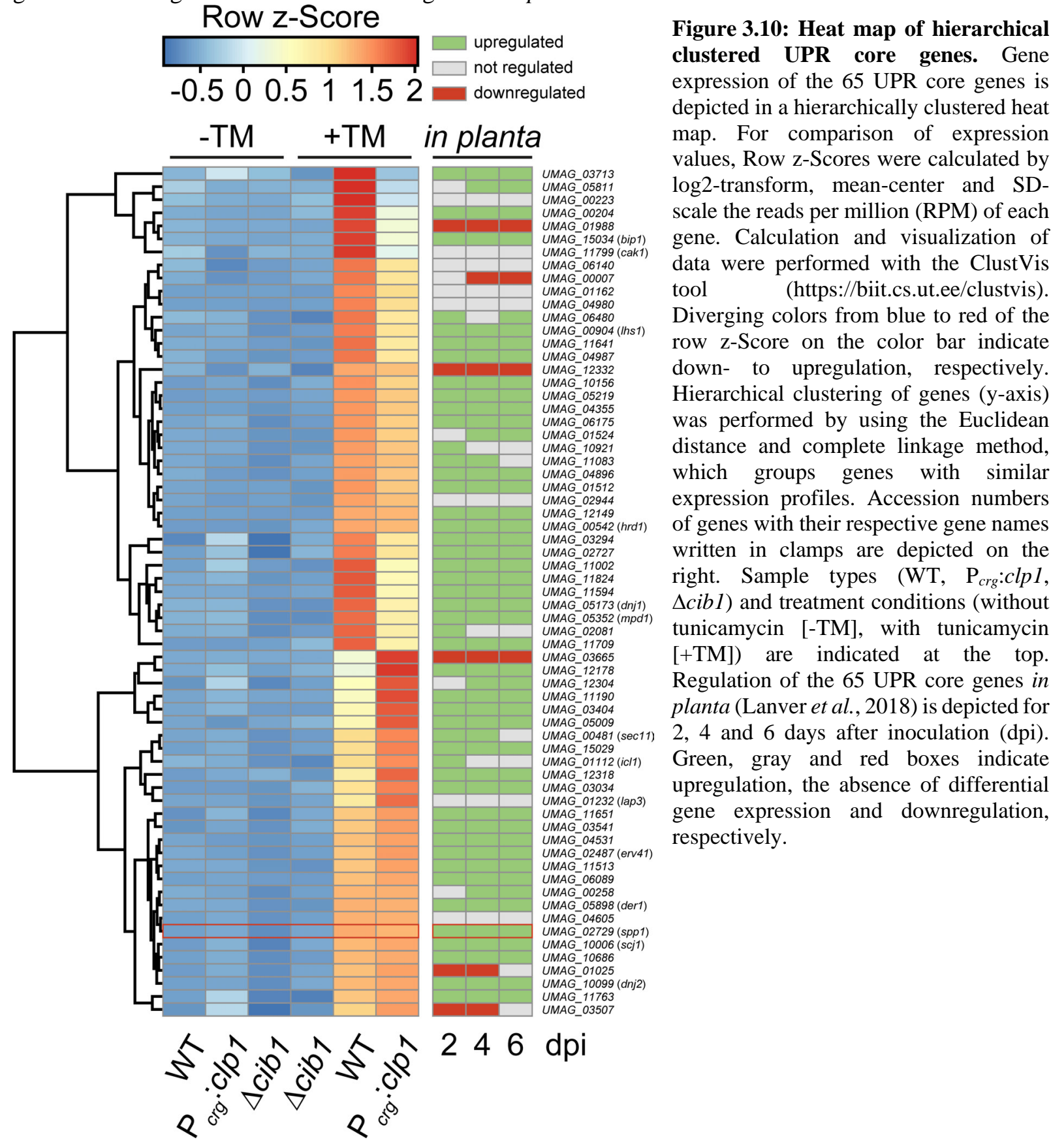


At 6 dpi, 15 UPR core genes are not regulated and 4 are downregulated in planta (Fig 3.10). Three of the 65 UPR core genes (UMAG_00223, UMAG_01988, UMAG_11799) are upregulated during UPR activation (WT +TM), but not upregulated in the clp1 expressing strain $\left(\mathrm{P}_{\text {crg }}: c l p 1+\mathrm{TM}\right)$ and in planta. This indicates a correlated gene expression between RNAseq data obtained from axenic culture under ER stress and plant-derived transcriptomic data. Furthermore, expression of the genes UMAG_12332, UMAG_02944,UMAG_04605, UMAG_01025 and UMAG_03507 is upregulated during UPR activation $(\mathrm{WT}+\mathrm{TM})$ as well as in clp1 expressing strain $\left(\mathrm{P}_{\text {crg:clp1 }}+\mathrm{TM}\right)$, but not upregulated in planta. The genes of a third group (UMAG_03665, UMAG_01232) are upregulated in the clp1 expressing strain ( $\mathrm{P}_{\text {crg:clp1 }}$ +TM) compared to the wildtype strain (WT +TM), but not upregulated in planta (Fig 3.10).

To visualize Clp1-dependent effects on the UPR core genes, log2 fold changes of the treated JB1 strain

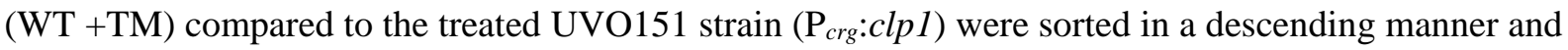
divided into six groups. 35 UPR core genes are not repressed by clp1 and only 3 of these 35 genes were upregulated more than 2-fold $(\log 2 \mathrm{FC} \geq 1)$. However, $30 \mathrm{UPR}$ core genes were not or downregulated by clp1 induction (log2FC $\leq-0.1$ ). 23 of these genes have a log2 fold change value between -0.1 and 1.0. Of the 30 UPR core genes, 5 genes are more than 2-fold downregulated ( $\log 2 \mathrm{FC} \leq-1.0$ to -2.0 ) and 2 genes are more than 4 -fold $(\log 2 \mathrm{FC} \leq-2.0)$ negatively regulated by the $c l p 1$ induction (Fig 3.11). The Clp1-dependent modulation of the UPR core gene expression in axenic culture indicates, that this alteration might also be important for in planta development of $U$. maydis.

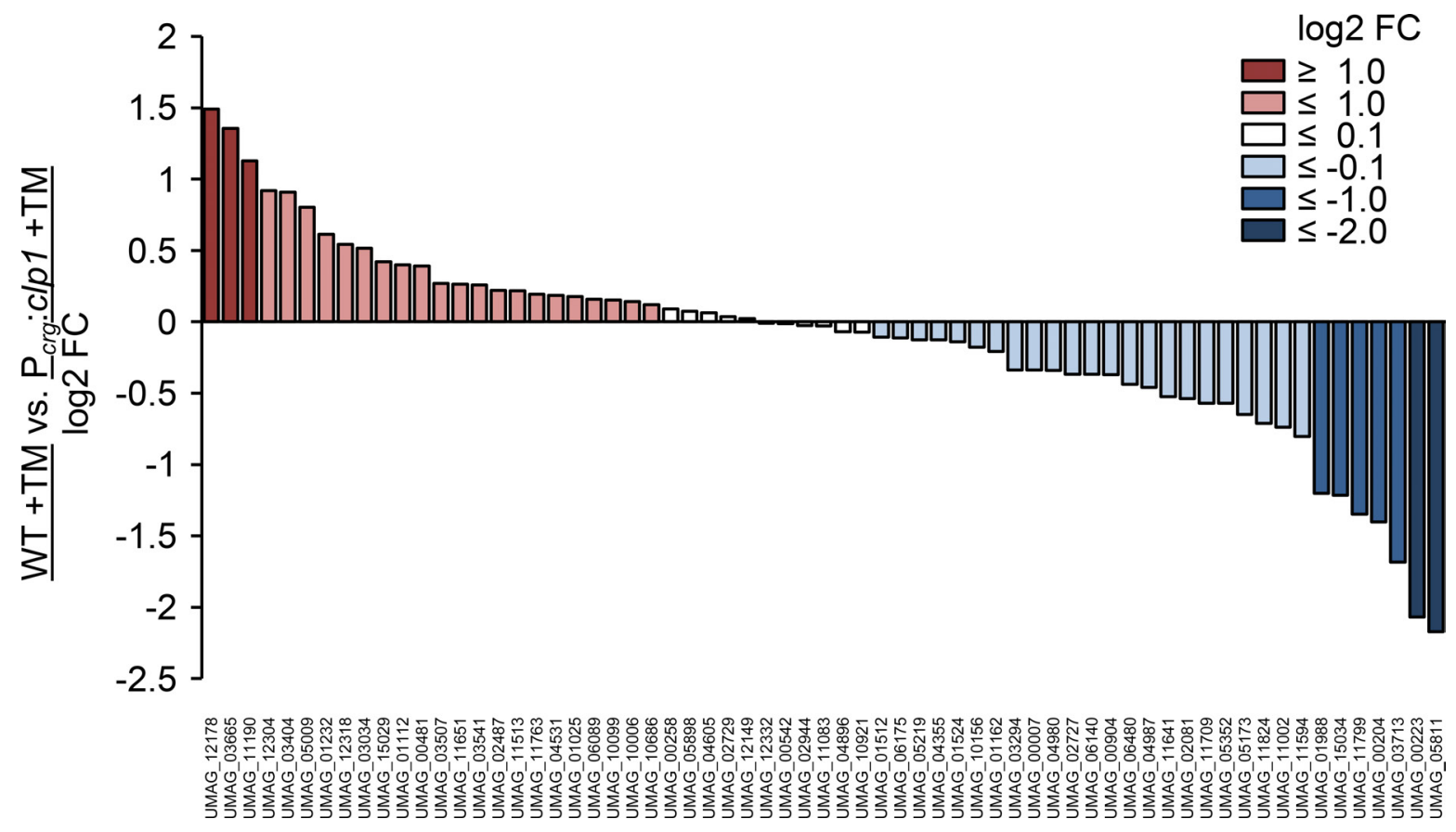

Figure 3.11: Clp1-dependent modulation of UPR core genes. Expression values of UPR core genes were log2 transformed and fold changes were calculated by comparison of the wildtype strain (WT) with the clp1 expressing strain $\left(\mathrm{P}_{\text {crg }}\right.$ :clp1), both treated with TM (WT + TM vs. $\mathrm{P}_{\text {crg: }}$ :clp1 +TM). Genes were sorted in a descending manner by their $\log 2 \mathrm{FC}$ values. Red and blue colors of the bars are indicating increased or reduced expression in the

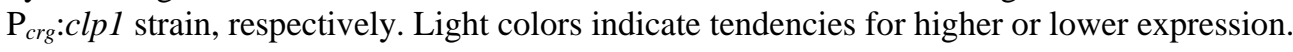




\subsubsection{Clp1 is dispensable for Cib1 DNA binding specificity}

In a previous study, a putative DNA binding motif of the bZip transcription factor Cib1 was predicted based on in silico promoter analysis and quantitative chromatin immunoprecipitation (qChIP) (Hampel et al., 2016). The determined consensus sequence of that study (TGCCACGT(C/G)(G/T)) is similar to the UPRE (UPR element) bound by the human or yeast Cib1 orthologs Xpb1 and Hac1, respectively (Fordyce et al., 2012; Kanemoto et al., 2005). To test if clp1-dependent modulation of UPR gene expression is connected to alterations of DNA binding specificity of Cib1, a comparative ChIPseq analysis was performed. To this end, U. maydis strains JB1cib1-3xHA (WT) and UVO151cib1-3xHA

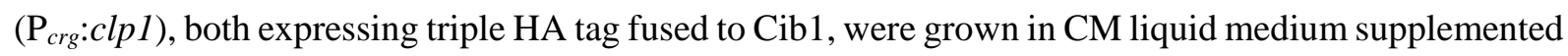
with $1 \%$ glucose (w/v) (CMG) to an $\mathrm{OD}_{600}$ of 0.35 . Subsequently, cells were shifted to $\mathrm{CM}$ liquid medium supplemented with $1 \%$ arabinose (w/v) (CMA) to induce $\mathrm{crg} 1$ driven promoter expression of clp1 and treated with TM for 4 hours at $28^{\circ} \mathrm{C}$, followed by chromatin-immunoprecipitation. DNA samples were submitted for library construction and subsequent sequencing in the single-end mode. Raw ChIPseq reads were aligned using Bowtie2 and peakZilla was used for peak calling. peakZilla provided a peak score ( $\Delta$ normalized reads (IP-input) $\mathrm{x}$ distribution score), which consolidates the probability of DNA binding with the DNA binding specificity. Identified ChIP peaks and corresponding promoter regions were manually assigned to genes using a custom python script (Appendix File 1). To allocate identified peaks in overlapping promoter regions, expression values (log2FC) of the RNAseq analysis were used for peak assignment. If more than one peak was assigned for a promoter, peak scores were accumulated to a promoter score (1.5 kb upstream of transcription start site (tss), with a minimal peak score of $\geq 40$ and a peak count/promoter cut-off of $<4$ ). In addition, assigned peaks were filtered with a promoter score cut-off of $\geq 100$. With this approach, 476 peaks were identified (Appendix Table 3), corresponding to 281 promoter regions in WT (JB1cib1-3xHA) and 654 identified peaks,

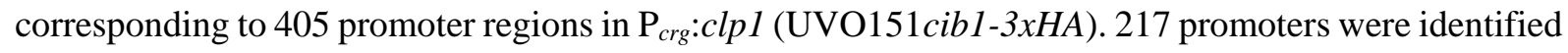
in both strains, whereas 63 and 188 are only identified in WT or $\mathrm{P}_{c r g}$ :clp1, respectively. 
Table 3.2: Top 20 candidates of promoters with the highest promoter score identified by ChIPseq.

\begin{tabular}{|c|c|c|c|c|}
\hline Rank & Name & $\begin{array}{c}\text { Accession } \\
\text { number }\end{array}$ & $\begin{array}{l}\text { Promoter } \\
\text { score }\end{array}$ & $\begin{array}{l}\text { Peak } \\
\text { count }\end{array}$ \\
\hline 1 & bip1 & UMAG_15034 & 922 & 1 \\
\hline 2 & cib1 & UMAG_11782 & 802 & 1 \\
\hline 3 & pdi1 & UMAG_10156 & 796 & 1 \\
\hline 4 & ero1 & UMAG_05219 & 783 & 2 \\
\hline 5 & - & UMAG_04980 & 553 & 1 \\
\hline 6 & cak1 & UMAG_11799 & 488 & 1 \\
\hline 7 & - & UMAG_02081 & 470 & 2 \\
\hline 8 & - & UMAG_05170 & 444 & 3 \\
\hline 9 & - & UMAG_11594 & 429 & 1 \\
\hline 10 & Ihs1 & UMAG_00904 & 417 & 1 \\
\hline 11 & - & UMAG_03038 & 399 & 3 \\
\hline 12 & $d n j 1$ & UMAG_05173 & 389 & 1 \\
\hline 13 & - & UMAG_03415 & 387 & 1 \\
\hline 14 & - & UMAG_02727 & 374 & 1 \\
\hline 15 & - & UMAG_01988 & 366 & 2 \\
\hline 16 & - & UMAG_12062 & 362 & 2 \\
\hline 17 & - & UMAG_10473 & 357 & 2 \\
\hline 18 & - & UMAG_05911 & 346 & 1 \\
\hline 19 & - & UMAG_01667 & 344 & 3 \\
\hline 20 & - & UMAG_04998 & 339 & 3 \\
\hline
\end{tabular}

Within the top twenty candidates of promoters with the highest promoter score (Table 3.2), promoters of the known UPR genes bip1, cib1, pdi1, ero1, lhs1 and dnj1 could be identified (Appendix Table 3). Promoter regions of assigned ChIPseq peaks were used for a motif-based analysis using the MEME (Multiple Em for Motif Elicitation)-ChIP web service (Machanick and Bailey, 2011). Exclusively

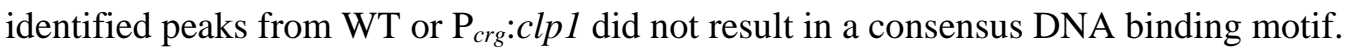

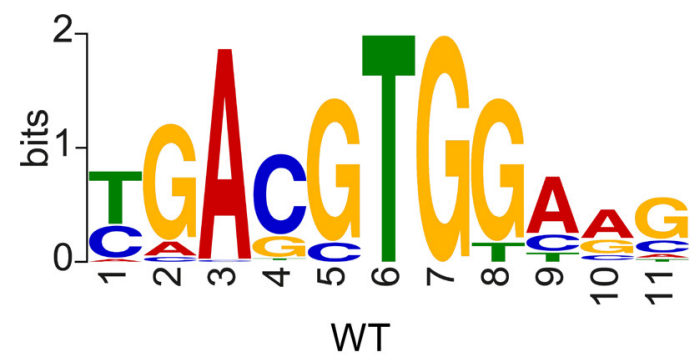

E-Value: $4.6 \times 10^{-44}$

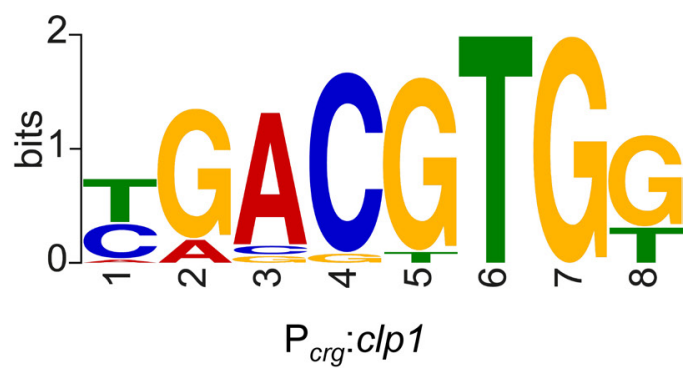

E-Value: $9.9 \times 10^{-47}$

Figure 3.12: Cib1 DNA binding specificity is not altered upon Clp1 induction. For identification of centrally enriched DNA consensus motifs, FASTA files were generated from peaks in the 91 UPR gene promoters, which were present in wildtype (WT) and clp1 expressing condition ( $\mathrm{P}_{\text {crg: }}$ :lp1). Files were subjected to MEME-ChIP analysis. The probability of nucleotide-occurrence in the consensus motif is represented by the height of the letters compared to the entire motif. Stacked letters indicate that more than one nucleotide can occur at the position in the motif. E-values were calculated based on the log likelihood ratio, width, sites, the background letter frequencies and the size of the training set.

MEME-ChIP analysis of UPR core gene promoters, bound by Cib1 in WT and $\mathrm{P}_{\text {crg }}$ :clp1 $(\log 2 \mathrm{FC} \geq 1$, $\mathrm{n}=91$ promoters), revealed a centrally enriched, 11 bp CREB3-like binding motif in the WT strain (Fig 3.12, WT, E-value: $4.6 \times 10^{-44}$ ). The motif occurred 63 times within the 91 promoter regions of the WT strain. For the clp1 expressing $\mathrm{P}_{\text {crg: }}$ :clp1 strain, an almost identical 8 bp motif was identified 


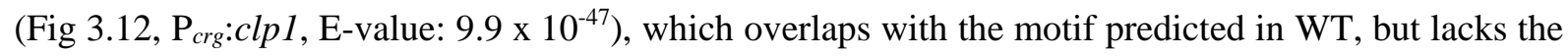
terminal AAG triplet. This motif occurred 88 times within the 91 promoter regions of the $\mathrm{P}_{\text {crg:clp}} 1$ strain.

The Integrative Genome Viewer (IGV, (Robinson et al., 2017)) was used to visualize ChIPseq peaks and revealed that that peak shapes and peak locations were conserved between WT and $\mathrm{P}_{\text {crg:clp1 }}$ (Fig 3.13). Differences in the peak size of the known UPR target genes cib1, bip1, ero1, pdi1 and spp1

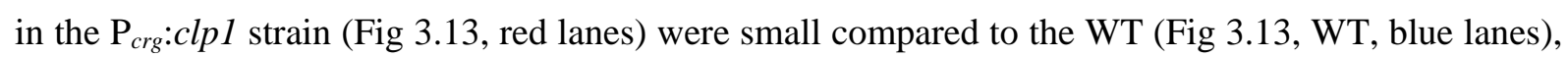
except for cak1 promoter, where the peak height was increased in the clp1 expressing condition (Fig 3.13, cak1, $\mathrm{P}_{\text {crg: }}$ :clp1). For promoters of cib1, bip1, ero1 and pdi1 and spp1 peak sizes correlate with expression values of the RNAseq analysis. By contrast, the peak size of the cak1 promoter is inversely correlated with its expression values (Fig 3.13, RPKM inserts).

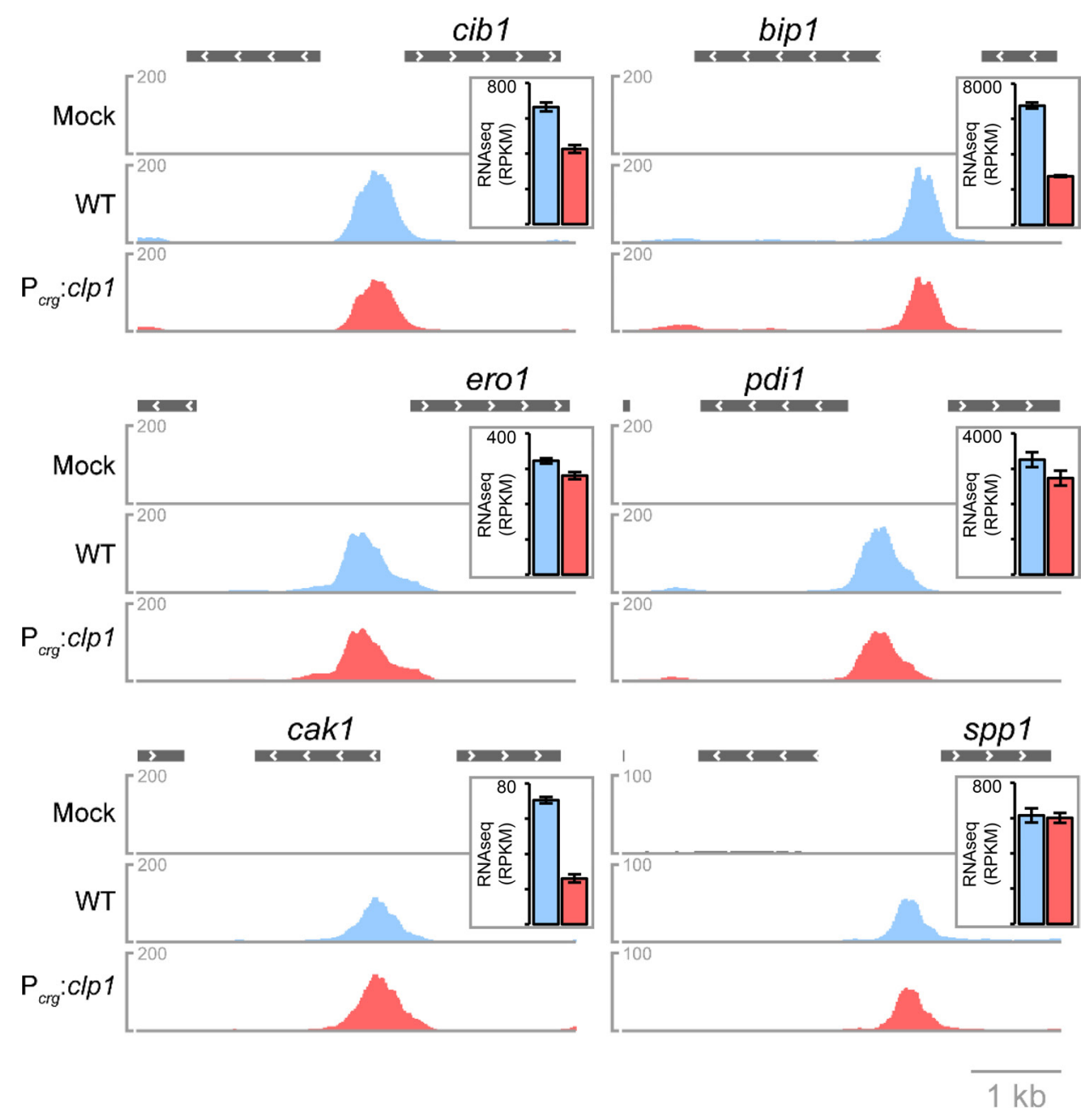

Figure 3.13: Visualization of ChIP peaks in known UPR target genes. For visualization of ChIP peaks, normalized BigWig data files obtained from raw ChIPseq data were used and illustrated with the Integrative Genome Viewer (IGV). Peak profiles are represented by normalized read counts derived from two biological replicates in JB1cib1-3xHA (blue) and UVO151-cib1-3xHA (red) compared to the untagged mock control (gray). Gene expression determined by RNAseq analysis (Chapter 5.6.1) of respective genes is depicted in reads per kilobase of transcript, per million mapped reads (RPKM) as a mean of three biological replicates (insets). Error bars depict the SD. Scale bar corresponds to $1 \mathrm{~kb}$.

Overall, the identified peaks in 91 UPR promoters $(\log 2 \mathrm{FC} \geq 1)$ used for the MEME-ChIP analysis

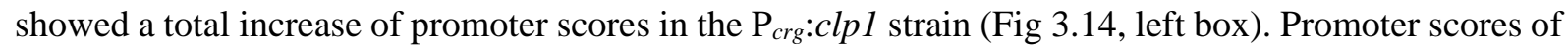


19 genes were lower, whereas promoter scores of 69 genes were higher during clp1 induction compared to wildtype. This correlation is explicitly pronounced for peaks with low promoter scores.

However, for high scoring peaks (Fig 3.14), as retrieved for the promoters of bip1, ero1, pdi1 or cib1

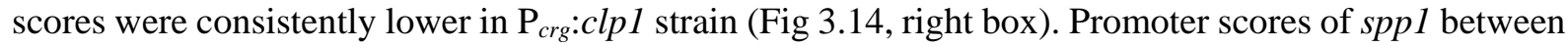
both strains were almost identical (Fig 3.14, left box, WT: 260.82 vs. $P_{c r g}: c l p 1: 261.41$ ). Taken together, these results show that $c l p 1$ induction does not affect DNA binding specificity of Cib1 (Fig 3.12 and 3.13) but differentially affects promoter scores, that indicates a post-translational mechanism that might affect the binding affinity of Cib1. Moreover, the identified Cib1-binding site (Fig 3.12, WT) perfectly matches to the promoter regions of pit1/2 and tin1-1 (Hampel et al., 2016). However, peak enrichment in the ChIPseq analysis was only detectable for the tin1-1 promoter (Appendix Fig 7.1).

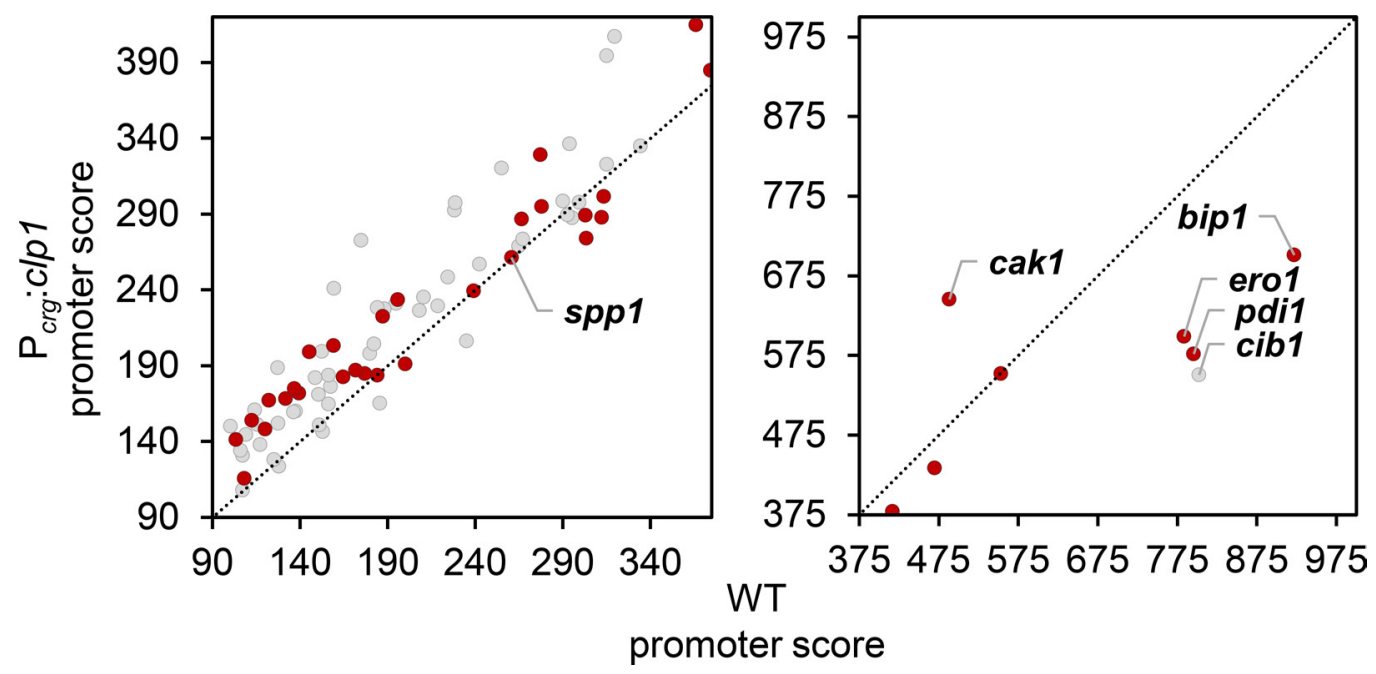

Figure 3.14: Comparison of promoter scores derived from ChIPseq analysis. Promoter scores of WT (JB1cib1-3xHA, $\mathrm{x}$-axis) and $\mathrm{P}_{c r g}$ :clp1 (UVO151cib1-3xHA, y-axis) were compared in a scatter plot, to visualize changes of promoter scores during clp1 expression. Peak scores were calculated by peakZilla (https://github.com/steinmann/peakzilla) and were accumulated to a promoter score if more than one peak was assigned to a promoter region (1.5 kb upstream of transcription start site (tss), with a minimal peak score of $\geq 40$ and a peak count/promoter cut-off of $\geq 4$ ). Only genes with promoter scores $\geq 100$ and gene expression of $\log 2 \mathrm{FC}$ $\geq 1$ were used for comparison. UPR core genes $(\log 2 \mathrm{FC} \geq 2)$ and UPR regulated genes $(\log 2 \mathrm{FC} \geq 1)$ are indicated as red and gray dots, respectively. 


\subsection{Deletion of UPR core genes identifies a novel pathogenicity factor}

The RNAseq analysis described in chapter 3.2.1 revealed a set of upregulated UPR core genes that are induced by ER stress in a cib1 dependent manner. Furthermore, the differential modulation of these UPR core genes by clp1 expression was observed (Fig 3.10 and 3.11). Most of the fungal effector proteins in $U$. maydis are highly upregulated during biotrophic growth (Lanver et al., 2018). Activation of the UPR after plant penetration marks a key step during pathogenic development, as it is important for effector secretion and expression (Heimel et al., 2013; Hampel et al., 2016). Thus, it was hypothesized that UPR core genes that are positively affected by clp1 expression might exert important functions during pathogenic development of $U$. maydis.

\subsubsection{Deletion of unrepressed UPR genes had no major impact on pathogenicity and ER stress resistance}

54 of the 65 UPR core genes (Fig 3.8), were modulated by clp1 expression (Fig 3.10 and 3.11). 30 UPR core genes were downregulated, whereas 11 genes were not regulated and 24 genes were upregulated during clp1 expression. It was assumed that unrepressed UPR core genes are important to establish biotrophic growth since expression of UPR regulated genes in planta described before is crucial for pathogenic development (Heimel et al., 2013; Hampel et al., 2016). To test this assumption, deletion mutants of the UPR core genes were generated in the solopathogenic strain SG200 (Bölker et al., 1995). Only 29 of 35 genes were investigated in this infection screen, as 3 gene deletions turned out to be lethal (UMAG_15029, UMAG_00481 and UMAG_06089) and 3 deletion strains were investigated before (UMAG_03034 [pers. communication Jörg Kämper, no phenotype], UMAG_04531 [pers. communication Regine Kahmann, no phenotype] and UMAG_10099 [Hampel (2016), reduced virulence]). All 29 deletion strains were tested in plant infection experiments and ER stress assays, to test for a role of the deleted genes in virulence and ER stress resistance. Of the 29 deletion mutants, only two gene deletions resulted in slightly reduced virulence compared to the wildtype strain (Fig 3.16, $\triangle U M A G \_11083$ and $\triangle U M A G \_12178$ ) and none of the deletion strains showed altered ER stress resistance. Importantly, deletion of UMAG_02729, which encodes a signal peptide peptidase, resulted in the complete loss of virulence, as reflected by the absence of more tumor formation and only leaf chlorosis symptoms (Fig 3.15). This suggests that the UMAG_02729 is a novel UPR regulated key factor with a crucial function during pathogenic development of $U$. maydis. 

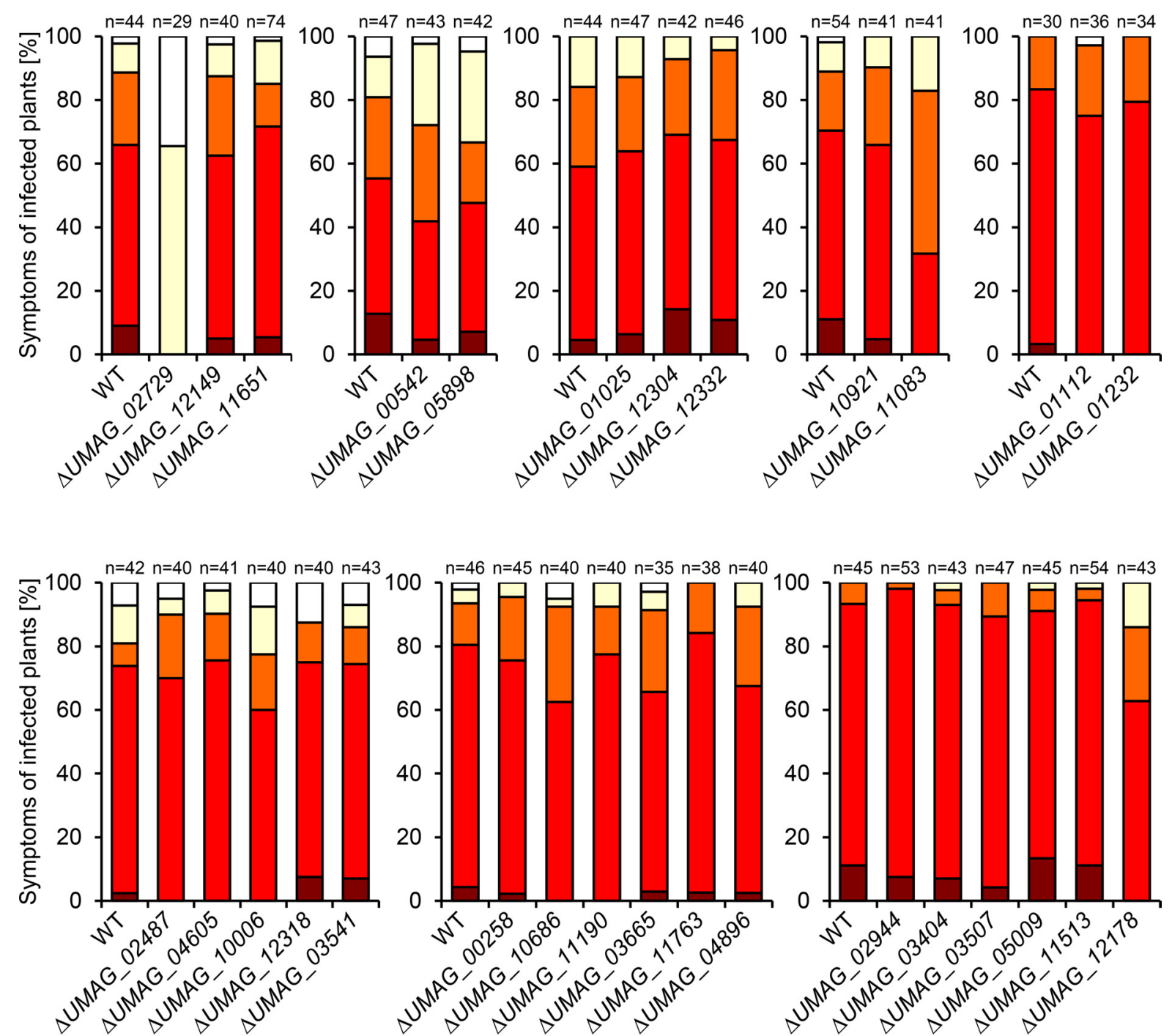

heavy tumors

normal tumors

small tumors

chlorosis

no symptoms

Figure 3.15: The UPR core gene UMAG_02729 is crucial for pathogenicity in $\boldsymbol{U}$. maydis. U. maydis strain SG200 (WT) and derivatives were inoculated into 7-day-old maize seedlings. Disease symptoms were rated $8 \mathrm{~d}$ after inoculation and grouped into categories depicted below. $n$ represents the number of inoculated plants in a single infection experiment. Data partially generated in (Hach, 2018). 

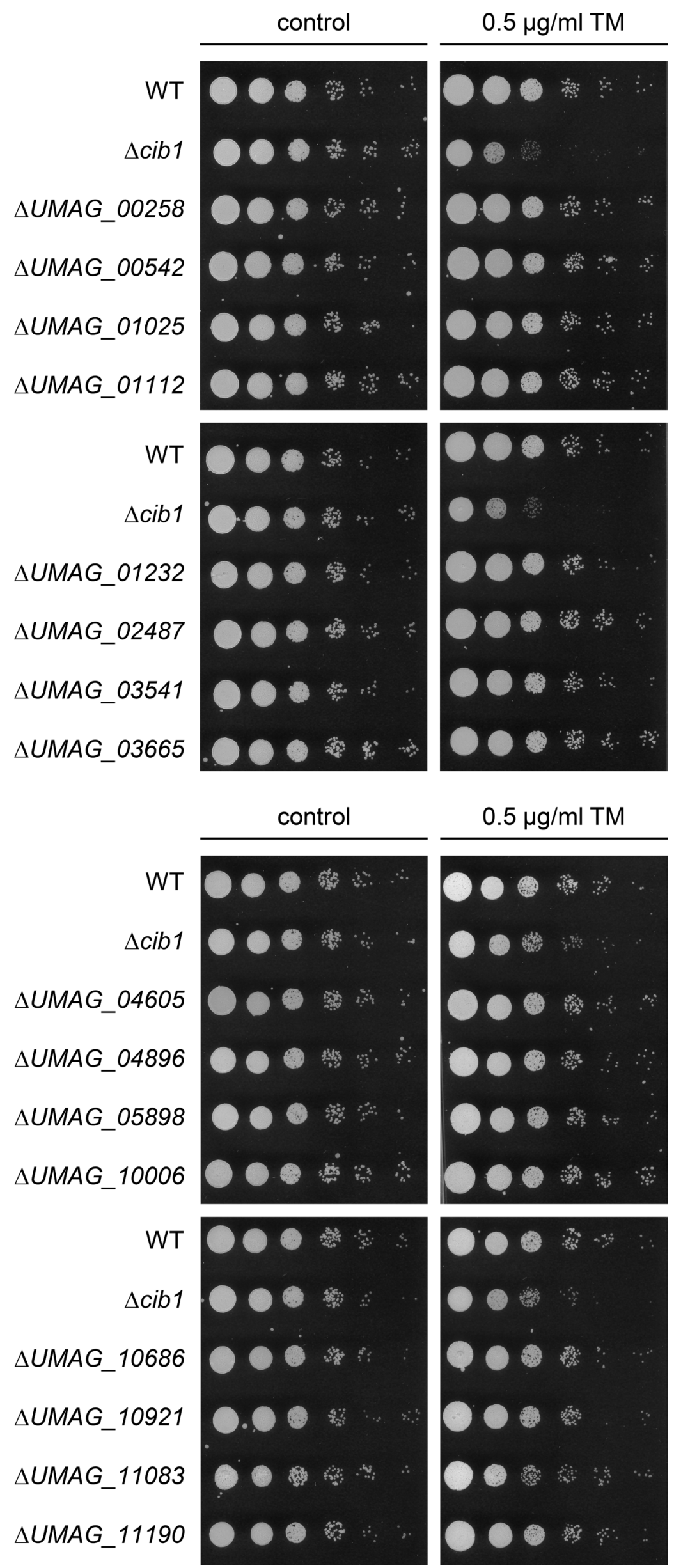


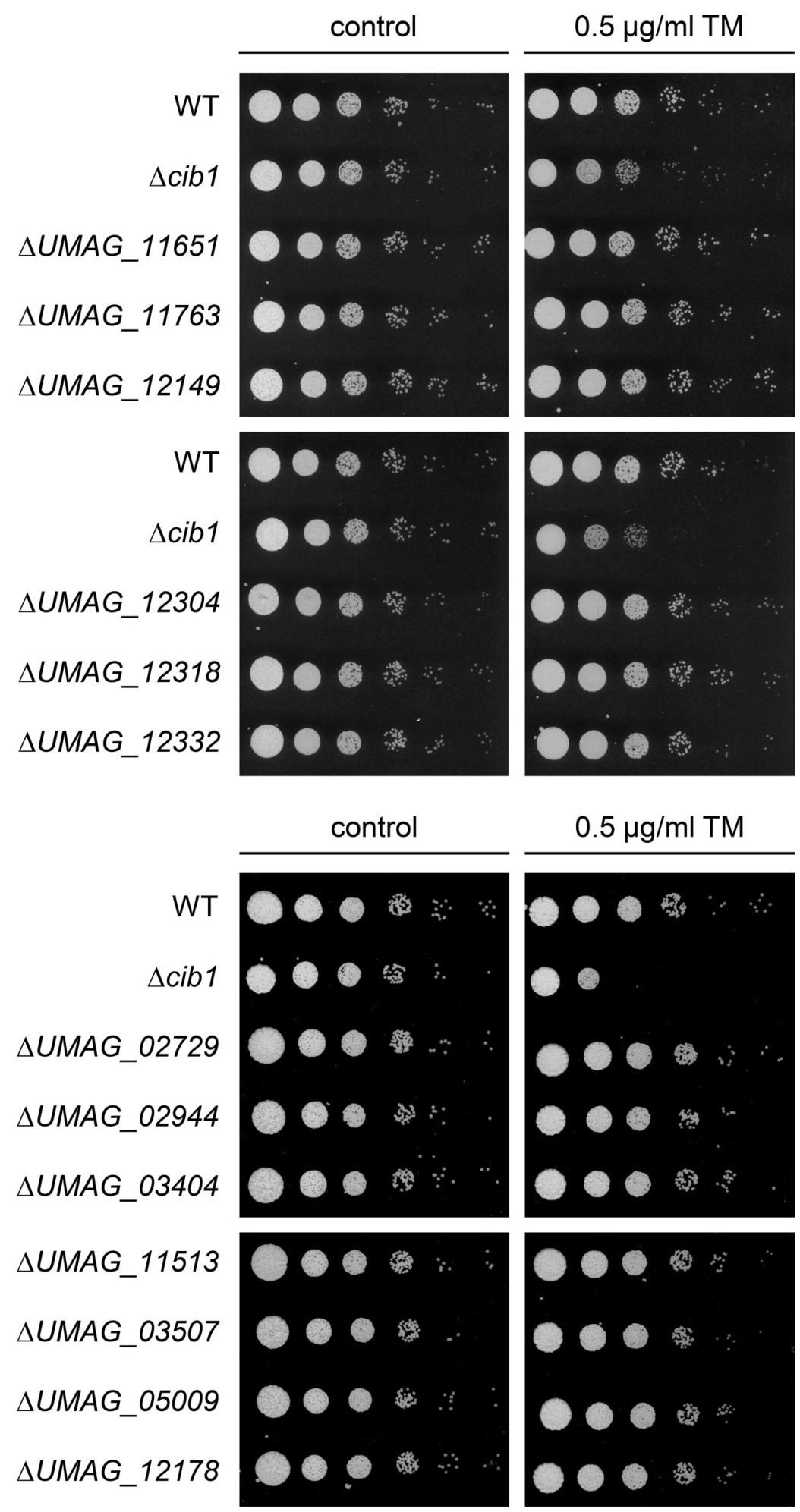

Figure 3.16: UPR core genes are not involved in ER stress tolerance. ER stress assay of $U$. maydis strain SG200 (WT) and derivatives. Serial 10-fold dilutions were spotted on YNBG solid medium supplemented with TM $(0.5 \mu \mathrm{g} / \mathrm{ml}$ f.c. $)$ as indicated. Plates were incubated for $48 \mathrm{~h}$ at $28^{\circ} \mathrm{C}$. Data partially generated in (Hach, 2018). 


\subsubsection{The UPR regulated gene UMAG_02729 encodes a Signal Peptide Peptidase}

Deletion of the gene UMAG_02729 resulted in loss of virulence (Fig 3.15) and gene expression of UMAG_02729 is highly induced by the UPR and in planta (Fig 3.10, red box). A BLASTp (Basic Local Alignment $\underline{\text { Search }}$ Tool) analysis (Altschul et al., 1990) of the protein sequence of UMAG_02729 was performed. This identified a conserved Presenilin family domain (E-Value $4.57 \times 10^{-34}$ ), characteristic for signal peptide peptidases (UMAG_02729 hereinafter referred to as spp1, signal peptide peptidase $\underline{1}$ ). BLASTp analysis of the protein sequence of Spp1 showed that Spp1 is the sole signal peptide peptidase in the genome of $U$. maydis. An analysis of the transmembrane topology with Phobius (Käll et al., 2004) predicted that the 416 amino acids (AA) long protein consists of 9 transmembrane domains (TMD). Neither SignalP (Petersen et al., 2011) nor Phobius could predict a signal peptide in the sequence of Spp1 (Fig 3.17).

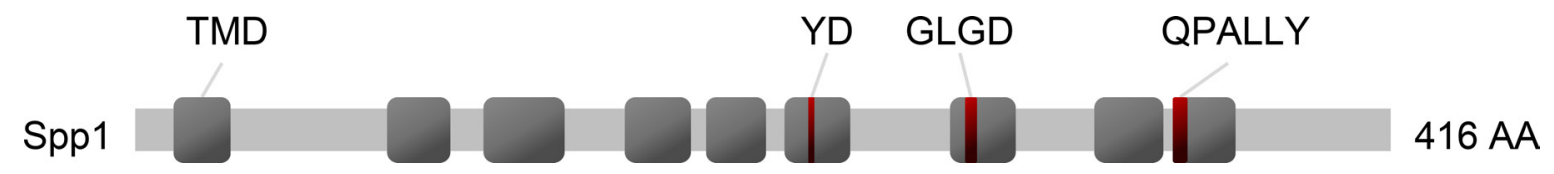

Figure 3.17: Schematic representation of the Spp1 domain structure. Gray boxes represent transmembrane domains (TMD). Red bars within TMDs mark the conserved YD, GLGD and QPALLY motifs. The protein has a total length of 416 AA.

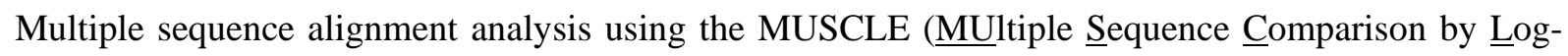
Expectation) algorithm (Chojnacki et al., 2017) and BLASTp was used to identify orthologues proteins of Spp1 in other organisms. For phylogenetic analysis orthologous proteins identified in the smut fungi Sporisorium reilianum (SrSpp1, sr13785, E-Value 0.0) and Ustilago hordei (UhSpp1, UHOR_04354, E-Value 0.0), as well as Homo sapiens (HM13, BC062595, E-Value $2 \times 10^{-62}$ ), the human pathogen and cause of malaria disease Plasmodium falciparum (PfSPP, PF3D7_1457000.1, E-Value $1 \times 10^{-44}$ ) and the ascomycetes Aspergillus nidulans (SppA, AN8681.2, E-Value $8 \times 10^{-38}$ ), Aspergillus fumigatus (SppA, XP_747862.1, E-Value 5 x 10-29) and Saccharomyces cerevisiae (Ypf1p, AJS50108.1, E-Value 4 x 10${ }^{31}$ ) were selected. Orthologs of the group of smut fungi showed the highest similarity to Spp1, with S. reilianum SrSpp1 of 0.06 substitutions per site (sps) and $U$. hordei UhSpp1 of 0.08 sps. Surprisingly, the H. sapiens HM13 and P. falciparum PfSPP were closer related to Spp1 with 0.67 sps and 0.79 sps, respectively, as compared to orthologs from ascomycetes, with A. nidulans SppA of 0.89 sps, A. fumigatus SppA of 0.94 sps and S. cerevisiae Ypf1p of 1.19 sps (Fig 3.18). 


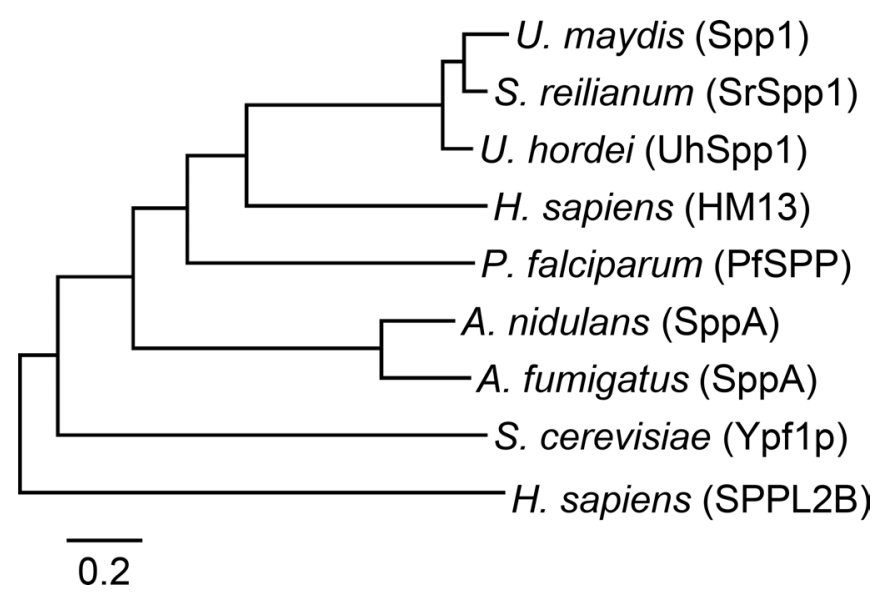

Figure 3.18: Spp1 is closely related to the human SPP HM13. Phylogenetic tree of U. maydis Spp1 and predicted orthologs from Sporisorium reilianum (SrSpp1), Ustilago hordei (UhSpp1), Homo sapiens (HM13), Plasmodium falciparum (PfSPP), Aspergillus nidulans (SppA), Aspergillus fumigatus (SppA) and Saccharomyces cerevisiae (Ypf1p). H. sapiens SPPL2B (Signal peptide peptidase-like 2B) was used as an outgroup. Construction of the phylogenetic tree was performed using the MEGA X software (https://www.megasoftware.net) by the Maximum Likelihood method based on sequence alignment by the MUSCLE algorithm of Figure 3.19.

The family of signal peptide peptidases shares known motifs for the proteolytic site and substrate binding of the intramembrane protease. The active site is represented by the YD and the GLGD motif, whereas substrate interaction is promoted through the QPALLY motif (Voss et al., 2013). In the multiple sequence alignment performed in Figure 3.19, both motifs of the proteolytic site can be found in all of the aligned sequences. However, the substrate binding motif differs in A. fumigatus, where it seems to be absent or is only weakly conserved in its sequence (Fig 3.19). The YD, GLGD and QPALLY motifs of $U$. maydis are located within the TMD6, TMD7 and TMD9, respectively (Fig 3.17 and Fig 3.19). 
U. maydis (Spp1)

S. reilianum (SrSpp1)

U. hordei (UhSpp1)

H. sapiens (HM13)

P. falciparum (PfSPP)

A. nidulans (SppA)

A. fumigatus (SppA)

S. cerevisiae (YPF1)

Conservation

U. maydis (Spp1)

S. reilianum (SrSpp1)

U. hordei (UhSpp1)

H. sapiens (HM13)

$P$. falciparum (PfSPP)

A. nidulans (SppA)

A. fumigatus (SppA)

S. cerevisiae (YPF1)

Conservation

. maydis (Spp1)

S. reilianum (SrSpp1)

U. hordei (UhSpp1)

$H$. sapiens (HM13)

$P$. falciparum (PfSPP)

A. nidulans (SppA)

A. fumigatus (SppA)

S. cerevisiae (YPF1)
A I ML GGLFV V Y D I I WWFATPV

A I MLGGLFVYY I FWVFATPV

A I MLGGLF VIYDII FWVFATPV

C I LLGGLF IIY DIVFWVFGTNV

FLLLSGLFV Y D,I FWVFGNDV

SL I LGSLFF'YD'I YFVYFTPL

SL I LGSLFLIYDII YF VFYTPL

AL I L I ALFFIYD:I YFVFGTDV

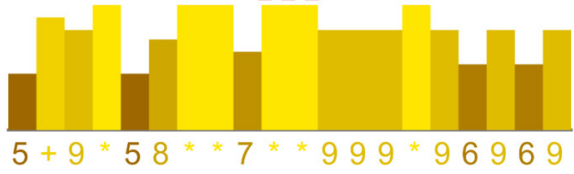

QPKLQF SMLIGLGDil V I PG I F VA

LPKLQF TML'GLGD'I V I PG I F VA

LPKLQF TML'GLGD'I V I PG I F VA

LEANNFAMLIGLGDNV I PG I F I A

- - - VHYSMLIGLG D'। I I PG I LMS

PGTVSLAML'GLGD'। I I PGMMVG

PDVVSLAML'GLGD'I V I PGMMVG

QNNFNFS I LIGLGD!'I ALPGMF I A

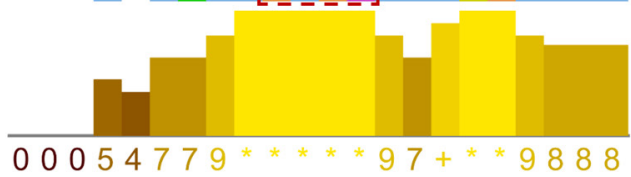

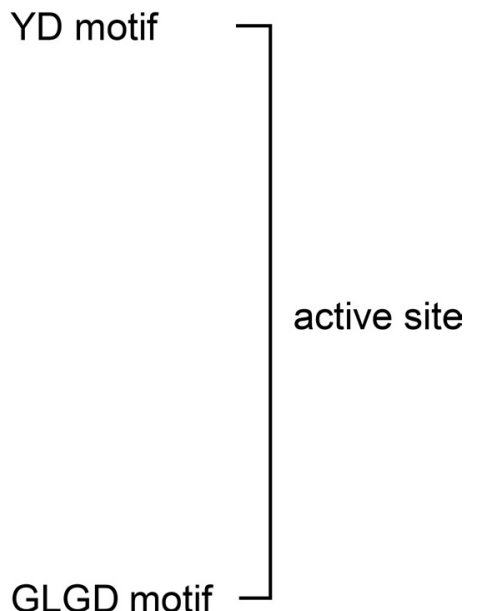

QPALLY motif

GLGD motif -

Conservation

G VMHFFQAAQPALL Y'LSPACTGAV

G VMHFFKAAQPALL YL SPACTGAV

GVMHFFKAA'QPALL YL SPACTGAV

$F$ I MH I FKHA'QPALL Y'L VPAC I GFP

CMLFYFEHPIQPALLYLVPAC I LA I

A VMQ I FDHPIQPALL YLVPG VL I SL

- . . . . - - PNL I SI-RPACLGTL

VSLS I FNTA'QPALL Y'I VPSLL I ST

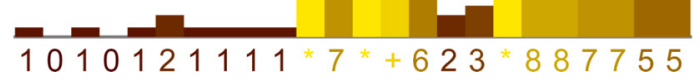

Figure 3.19: Spp1 is a conserved signal peptide peptidase. Protein sequences of $U$. maydis Spp1 and predicted orthologs from indicated species were aligned using the MUSCLE algorithm (https://www.ebi.ac.uk/Tools/msa/muscle) and visualized by JalView (http://www.jalview.org). Conserved sequence motifs are highlighted with red-dashed boxes. The YD and GLGD motifs represent the active site of the aligned signal peptide peptidases. The QPALLY motif is conserved in all sequences except for Aspergillus fumigatus. Conservation rate of the sequence is shown in the histogram below, where "+" and “*” representing a conservation rate of 10 and 11 , respectively. The Clustal X color scheme was used to group amino acids with similar properties. Full alignment is shown in Appendix Figure 7.2.

\subsubsection{Spp1 is localized to the perinuclear and cortical ER}

The bioinformatic analysis described in chapter 3.3.2 predicted several transmembrane domains in the sequence of Spp1. In addition, studies of orthologues proteins showed a localization at the ER membrane (Weihofen et al., 2002; Hsu et al., 2018; Avci et al., 2014). Spp1 was C-terminally tagged with mCherry and subcellular localization was monitored by fluorescence microscopy, to check the intracellular 
localization of Spp1 in U. maydis. The spp1 gene was expressed under the native promoter $\left(\mathrm{P}_{\text {spp } 1}:\right.$ spp1-

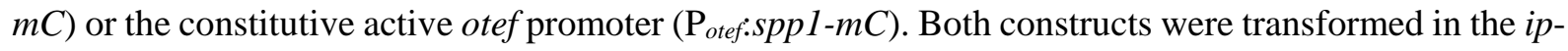
locus of the SG200Aspp1 strain (WT). Moreover, deletion of the UPR regulator cib1 in the resulting strains was performed ( $\Delta c i b 1)$, to compare the localization of Spp1-mCherry during UPR activation between WT and $\Delta c i b 1$. To induce the UPR individual strains were grown in CM liquid medium supplemented with $1 \%$ glucose $(\mathrm{w} / \mathrm{v})$ to an $\mathrm{OD}_{600}$ of 0.35 and treated with $\mathrm{TM}$ for 2 hours at $28^{\circ} \mathrm{C}$.

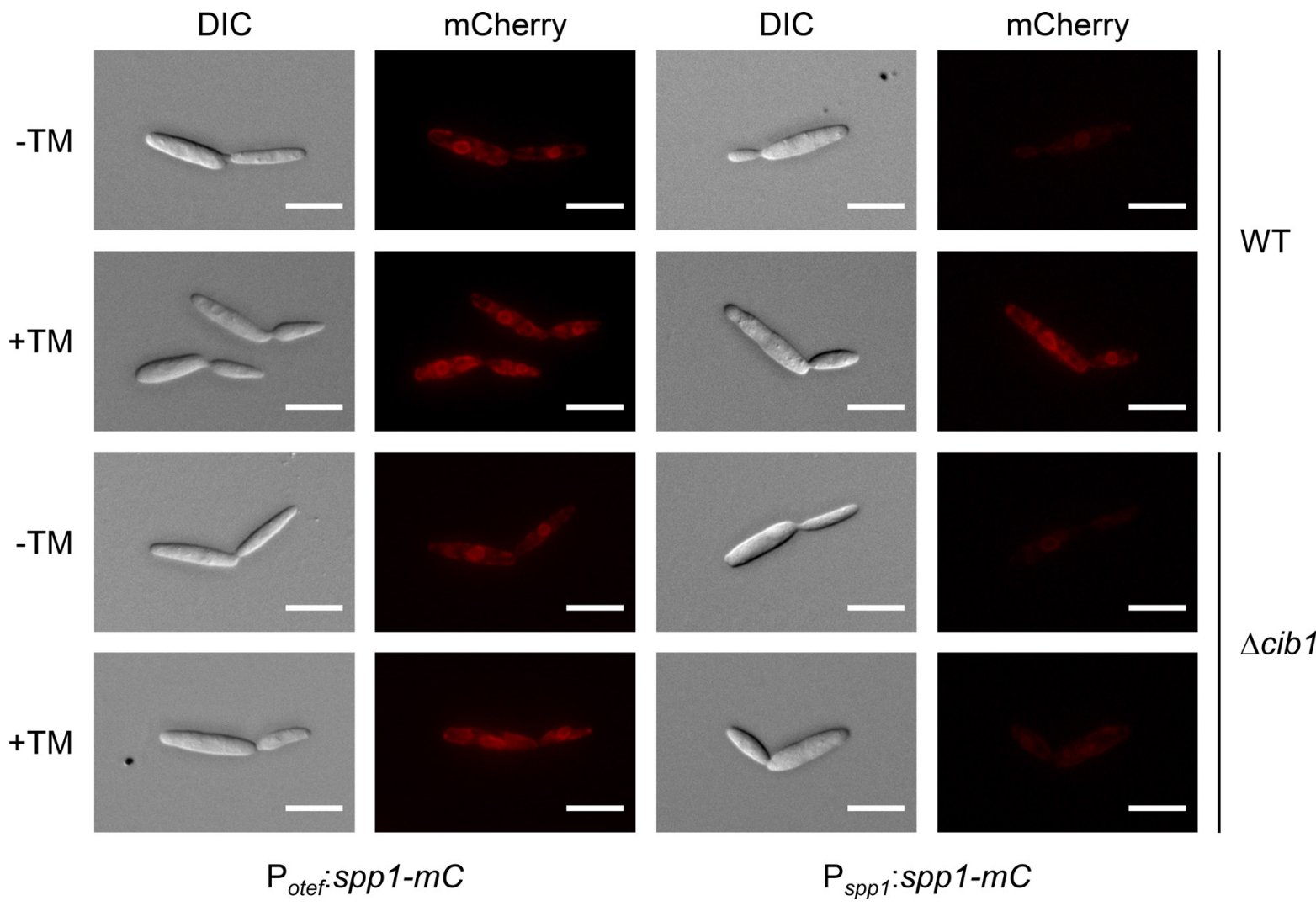

Figure 3.20: Spp1 is localized at the ER membrane and induced during ER stress. Expression of the Spp1mCherry fusion protein was monitored by fluorescence microscopy $2 \mathrm{~h}$ after TM-mediated UPR induction in the

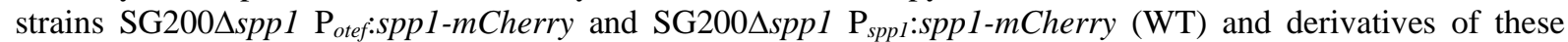
strains with an additional cib1 deletion ( $\Delta c i b 1)$. Exposure time of RFP channel was set to $750 \mathrm{~ms}$. Cellular morphology was visualized by DIC microscopy. Scale bars $=10 \mu \mathrm{m}$.

In the strains where Spp1-mCherry was expressed under the otef promoter, a bright fluorescence signal could be observed at structures that presumably correspond to cortical and perinuclear ER structures. Differences in intracellular localization or fluorescence signal intensity between the WT and $\Delta c i b 1$ strain backgrounds could not be observed, whether cells were treated with TM or not (Fig 3.20, Potef:spp1$m$ Cherry). However, in strains expressing Spp1-mCherry under its native promoter, only the TM treated

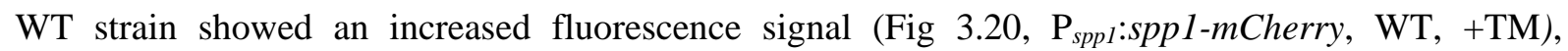
indicating an essential role of the UPR for induction of spp1 expression.

\subsection{4 spp1 is involved in the biotrophic growth in planta}

Since deletion of spp1 led to an apathogenic phenotype in the plant infection assay (Fig 3.15), the question aroused, at which stage of pathogenic development spp1 mutant strains are blocked. It was 
tested whether spp1 deletion mutants are capable of forming $b$-dependent filaments. Furthermore, the capability of spp1 deletions strains to invade the plant surface was analyzed. To investigate filament

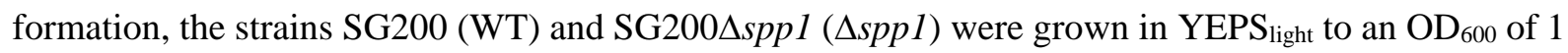
and spotted on potato dextrose (PD) charcoal medium. Formation of aerial hyphae was monitored on solid medium containing charcoal to mimic the plant's hydrophobic surface (Banuett and Herskowitz, 1988), which induces the $b$-dependent filament formation (Heimel et al., 2010b). The deletion mutant of spp1 (Fig 3.21, $\Delta$ spp1) showed no impairment in filament formation and no differences in the fuzzy appearance of the colony compared to the WT strain (Fig 3.21, WT).
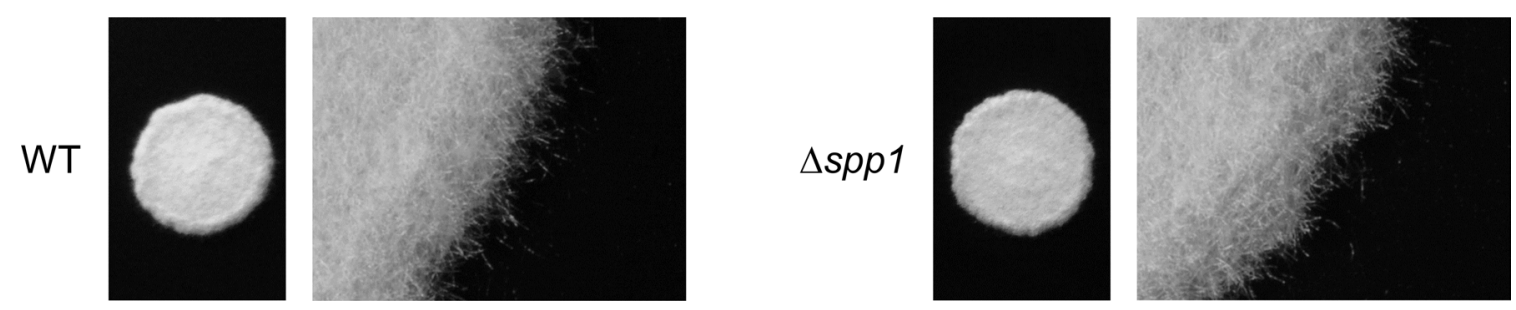

Figure 3.21: Filament formation is not impaired in spp1 deletion strains. $U$. maydis strain WT and the $\Delta$ spp1 derivative were spotted on charcoal containing $(1 \% \mathrm{w} / \mathrm{v})$ potato-dextrose (PD) medium to induce filament formation. Plates were incubated for $24 \mathrm{~h}$ at $28^{\circ} \mathrm{C}$ and photographed. White fuzzy filaments indicate the formation of $b$-dependent filaments.

To examine, if the spp1 deletion mutant is able to penetrate the plant surface, the strains SG200 (WT) and SG2004spp1 ( $\Delta$ spp1) were grown in YEPS light to an $\mathrm{OD}_{600}$ of 1 and were injected into 7-day-old maize seedlings. Infected leaf tissue was collected 3 dpi and stained with Chlorazol Black E to visualize invading hyphae in planta (Brachmann et al., 2003). The deletion mutant was able to form appressoria (Fig 3.22, $\Delta$ spp1, asterisks) and can penetrate the plant surface (Fig 3.22, $\Delta$ spp1). However, intercellular hyphae of the deletion mutant displayed a highly reduced proliferation in planta, restricted to the epidermal layer, and altered cell morphology with collapsed structures. In addition, intercellular hyphae formed constrictions at sites of plant cell traversal, compared to the WT strain (Fig 3.22, $\Delta$ spp1, arrows). 

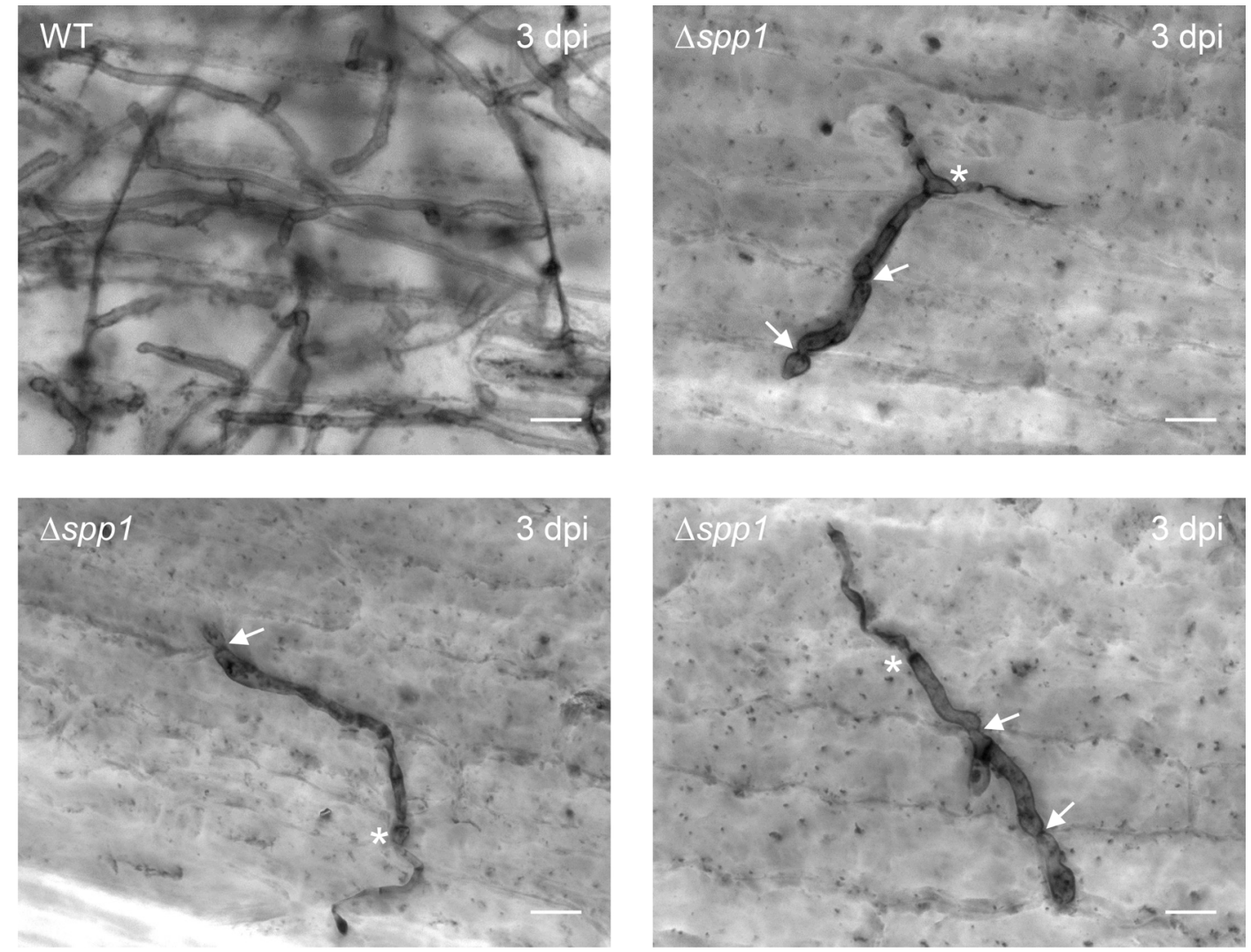

Figure 3.22: Deletion mutant of spp1 attenuated in growth after plant invasion. Fungal proliferation of WT and the $\Delta s p p 1$ was investigated by Chlorazol Black E staining of infected leaf samples $3 \mathrm{dpi}$. WT strains showed extensive proliferation in planta, whereas the $\Delta$ spp1 mutant showed strongly reduced proliferation after plant penetration. Asterisks mark the site of plant penetration and arrows indicate the points of plant cell traversal by fungal hyphae. Scale bar $=10 \mu \mathrm{m}$.

Since the overall plant colonization by the spp1 deletion mutant seemed to be lower (Fig 3.22), relative fungal biomass was measured and compared to the WT strain. For the analysis, the strains SG200 (WT),

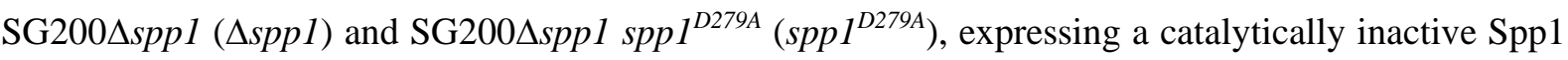
mutant, were grown in YEPS light to an $\mathrm{OD}_{600}$ of 1 and inoculated into 7-day-old maize seedlings. Infected leaf tissue was collected 2 and 4 dpi. At 2 dpi, the relative fungal biomass was significantly lower in the $\Delta$ spp1 (P-value $\leq 0.01$ ) and spp1 ${ }^{\text {D279A }}$ (P-value $\leq 0.01$ ) strain compared to the wildtype (Fig 3.23, 2 dpi) and even more reduced after $4 \mathrm{dpi}$ in the $\Delta s p p 1(\mathrm{P}$-value $\leq 0.01)$ and $s p p 1^{D 279 A}(\mathrm{P}$-value $\leq 0.05)$ strains (Fig 3.23, 2 dpi). This indicates that the spp1 is not involved in normal vegetative growth. However, strains are strongly impaired during in planta growth (Fig 3.22) and form almost no biomass at 4 dpi (Fig 3.23), indicating growth suppression of the $\Delta$ spp1 strain by an active plant defense response. 


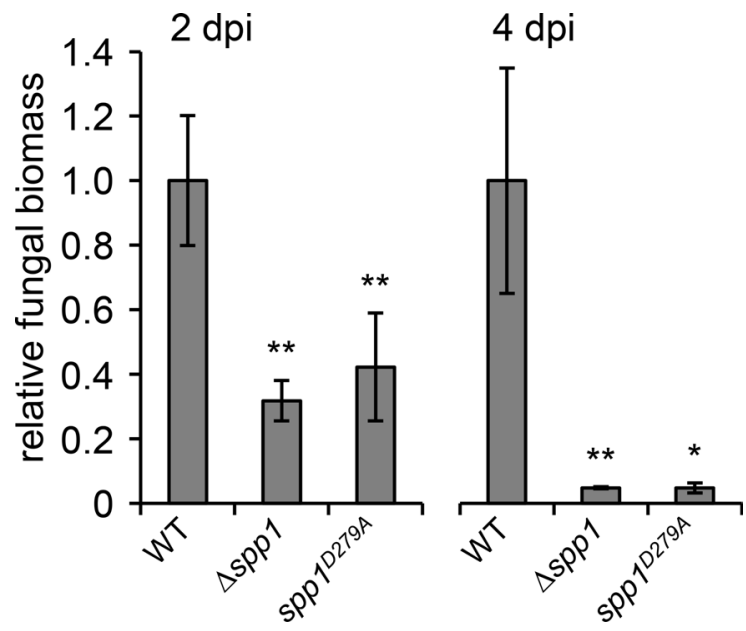

Figure 3.23: Spp1 function is crucial for growth in planta. Genomic DNA was extracted from maize seedlings inoculated with indicated strains at 2 and 4 dpi. Relative fungal biomass was calculated using the $U$. maydis specific $m f a 1$ gene as a fungal marker and the Zea mays specific glyceraldehyde 3-phosphate dehydrogenase gene $(G A P D H)$ as a plant marker. Values reflect the ratio of fungal/plant DNA relative to plants infected with the WT. Values represent the mean of three biological replicates with two technical duplicates each. Error bars represent the SD. Statistical significance was calculated using Student's $t$-test. ${ }^{* *} \mathrm{P}$-value $\leq 0.01$, ${ }^{*} \mathrm{P}$-value $\leq 0.05$. Data generated in (Hach, 2018).

\subsubsection{The $\Delta \operatorname{spp} 1$ phenotype can be suppressed by expression of Spp1 orthologs}

Orthologous proteins of Spp1 in the multiple alignment analysis described in chapter 3.3.2 showed highly conserved motifs throughout all analyzed species. To examine, if these orthologs of Spp1 can complement the deletion phenotype of spp1 in the SG200 strain, orthologous genes were integrated as a C-terminal mCherry fusion in the SG200Aspp1 strain and expressed under the control of the otefpromoter.

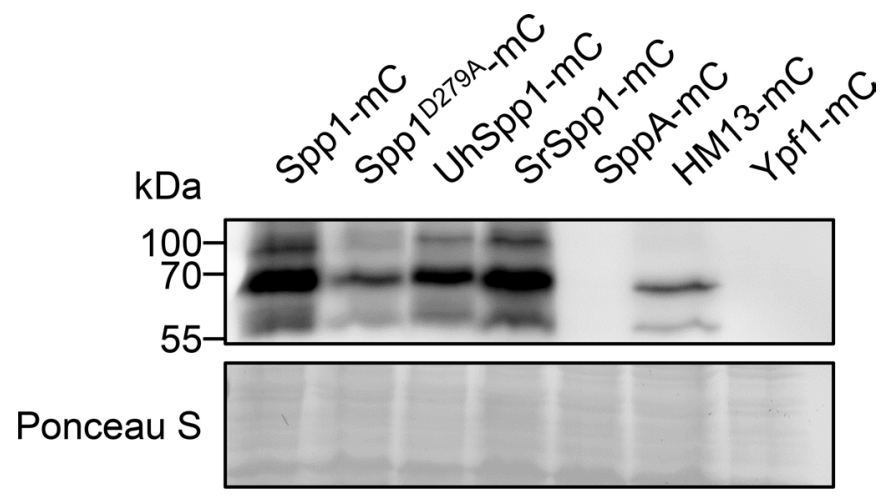

Figure 3.24: Western hybridization analysis of Spp1 mutants and orthologous proteins of Spp1. Expression of indicated fusion proteins was analyzed by Western hybridization in derivatives of U. maydis strain SG200_spp1. Proteins were expressed under the control of the constitutive active otef promoter. Protein extracts were prepared from exponentially growing cells cultured in CMG liquid medium. Ponceau S-stained membranes were used as loading control. No signal was detected for SppA-mC (A. nidulans) and Ypf1-mC (S. cerevisiae). Data generated in (Hach, 2018).

Western hybridization revealed successful expression of orthologs of spp1 from U.hordei (UhSpp1-mC), S. reilianum (SrSpp1-mC) and H. sapiens (HM13-mC) (Fig 3.24, lane 3, 4 and 6). 
Strains expressing the wildtype spp1 (Spp1-mC) from U. maydis, as well as the catalytically inactive mutant spp1 $1^{\mathrm{D279A}}\left(\mathrm{Spp}^{\mathrm{D} 279 \mathrm{~A}}-\mathrm{mC}\right.$ ) were used as controls (Fig 3.24, lane 1 and 2). By contrast, no expression was observed for strains harboring A. nidulans SppA-mC and S. cerevisiae Ypf1-mC (Fig 3.24, lane 5 and 7).

Plant infection assays were performed, to test if expression of orthologous proteins was sufficient to complement the deletion phenotype of spp1. The strain SG200 (WT) and derivatives were grown in YEPS $_{\text {light }}$ to an $\mathrm{OD}_{600}$ of 1 and were inoculated into 7-day-old maize seedlings. The strain with the integrated spp1-mC as well as the strains with orthologs of S. reilianum (srspp1-mC) and U. hordei (uhspp1-mC) could complement the $\Delta$ spp1 deletion phenotype. Surprisingly, the strain with a single copy of the human HM13-mC (HM13-mC (s)) was not able to recover the WT phenotype completely. In addition, a strain with multiple integrations of the HM13-mC construct HM13-mC (m)), was able to recover the phenotype of $\Delta s p p 1$ better than the strain with only one copy of the gene. Moreover, the catalytically inactive mutant (spp1 $1^{D 279 A}$ ) did not recover the phenotype of the spp1 deletion strain (Fig 3.25A and B). 
A
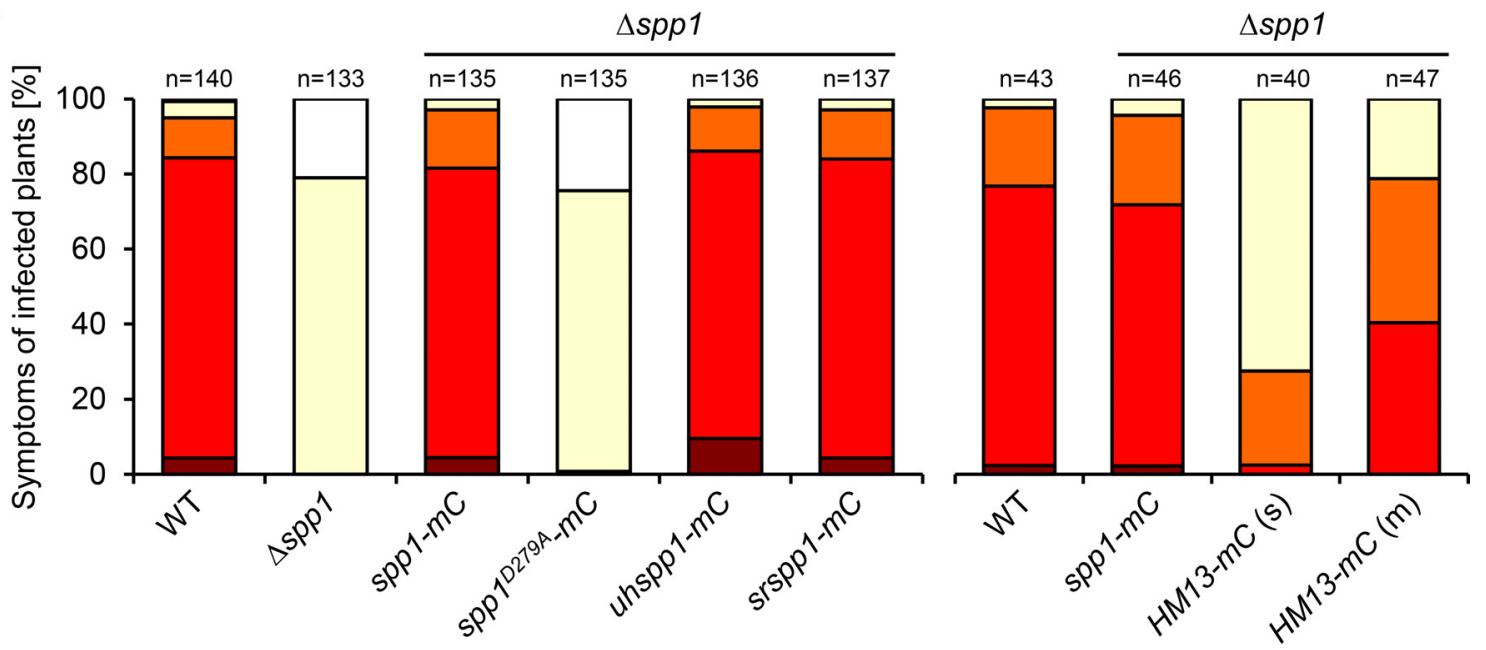

heavy tumors $\square$ normal tumors $\square$ small tumors $\square$ chlorosis $\square$ no symptoms

B
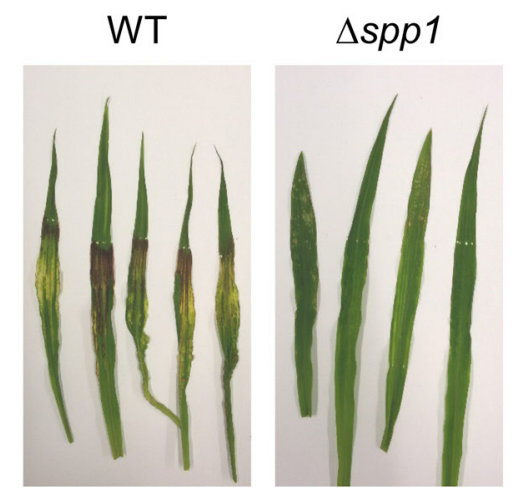

spp1-mC

spp $1^{D 279 A}-m C$
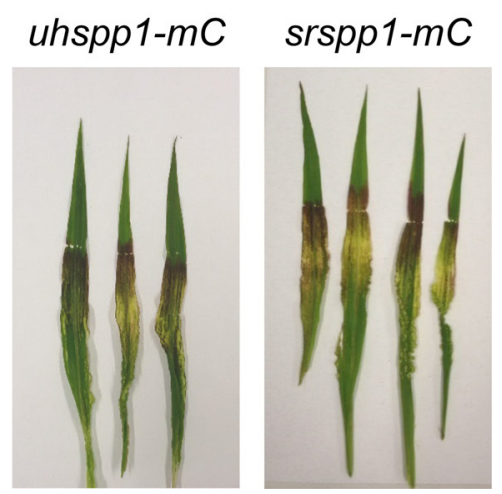

HM13-mC

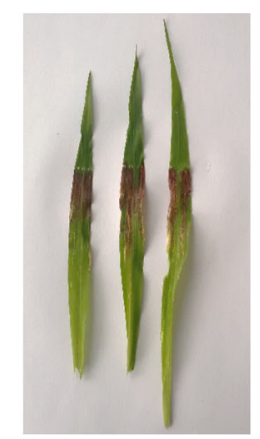

Figure 3.25: Orthologous spp1 genes could recover the spp1 deletion phenotype. (A) U. maydis strains SG200 (WT), SG200 $\Delta$ spp1 ( $\Delta$ spp1) and derivatives were inoculated into 7-d-old maize seedlings. In $\Delta$ spp1 derivatives, predicted orthologs or the catalytically inactive Spp1 ${ }^{\mathrm{D} 279 \mathrm{~A}}$ mutant protein were expressed as mCherry (mC) fusion under the control of the constitutively active otef promoter. Disease symptoms were rated at 8 dpi. $n$ represents the total number of inoculated plants derived from three (left) or two (right) independent experiments. For complementation tests using $H$. sapiens $H M 13, \Delta$ spp1 strains harboring single (s) and multiple (m) integrations of the HM13-mCherry (HM13-mC) fusion construct were used. (B) Leaf samples for macroscopic analysis were photographed at 8 dpi. Data partially generated in (Hach, 2018). 


\subsubsection{Spp1 represses defense responses in planta}

Deletion of spp1 led to a highly reduced proliferation in planta (Fig 3.22 and 3.23), with chlorosis as the strongest symptom in the plant infection assay (Fig 3.15 and 3.25A and B). As this might indicate an activation of the plant defense response, the strains SG200 (WT), SG200 $\Delta$ spp1 ( $\Delta$ spp1) and

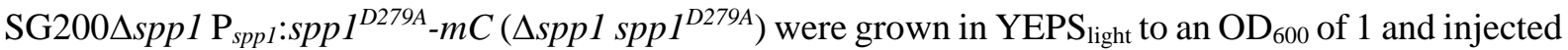
into 7-day-old maize seedlings and analyzed for reactive oxygen species (ROS) production. Infected leaf tissue was collected 24 hours post inoculation and stained with 3,3'-Diaminobenzidine (DAB) to visualize the formation of ROS in planta (Molina and Kahmann, 2007). Plants infected with the spp1 deletion mutant and the catalytically inactive Spp1 ${ }^{\mathrm{D} 279 \mathrm{~A}}$ mutant showed a strong accumulation of DAB when compared to the wildtype strain (Fig 3.26).
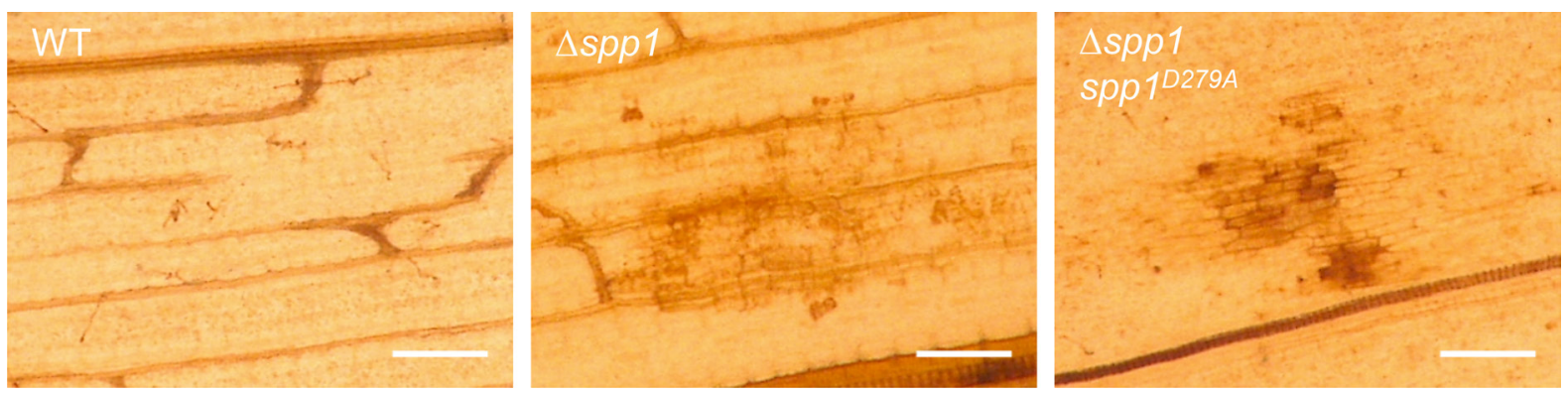

Figure 3.26: spp1 mutants elicited plant defense responses. 3,3'-Diaminobenzidine (DAB) staining of leaf tissue

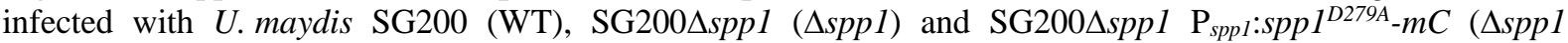
$s p p 1^{D 279 A}$ ) was performed $24 \mathrm{~h}$ post inoculation. Brown precipitates reflect the presence of reactive oxygen species (ROS). Scale bar $=100 \mu \mathrm{m}$. Data generated in (Hach, 2018).

ROS in plant cells are mainly derived from hydrogen peroxide (Bolwell and Wojtaszek, 1997). To test if the loss of virulence might be connected to hypersensitivity against ROS an oxidative stress assay

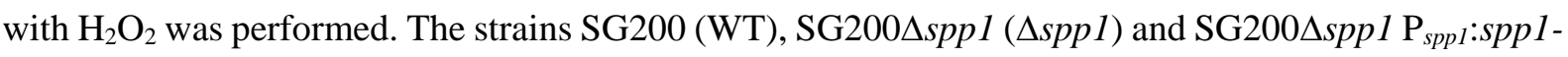
$m C$ ( $\Delta$ spp1 spp1-mC) were grown in YEPS light $_{\text {to an }} \mathrm{OD}_{600}$ of 1 and spotted on YNBG solid medium containing different concentrations of $\mathrm{H}_{2} \mathrm{O}_{2}$. After incubation for 48 hours at $28^{\circ} \mathrm{C}$, no differences could be observed between WT, $\Delta$ spp1 and $\Delta$ spp1 spp1-mC (Fig 3.27). Moreover, the strains SG200 (WT),

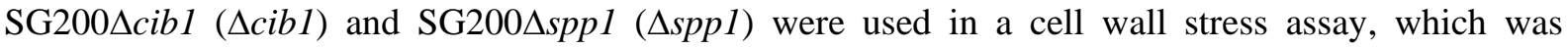
performed on solid medium containing either Congo Red or Calcufluor for cell wall stress. However, no differences in growth could be observed for the $\Delta c i b 1$ or the $\Delta$ spp1 strain compared to the wildtype (Appendix Fig 7.4). The increased ROS formation in plant infections with $U$. maydis indicates a strongly activated plant defense response. However, $\Delta$ spp1 strains are not susceptible to cell wall stress as well as $\mathrm{H}_{2} \mathrm{O}_{2}$ stress, which indicates that spp1 is not necessary for cell wall integrity or detoxification of $\mathrm{H}_{2} \mathrm{O}_{2}$, respectively. 


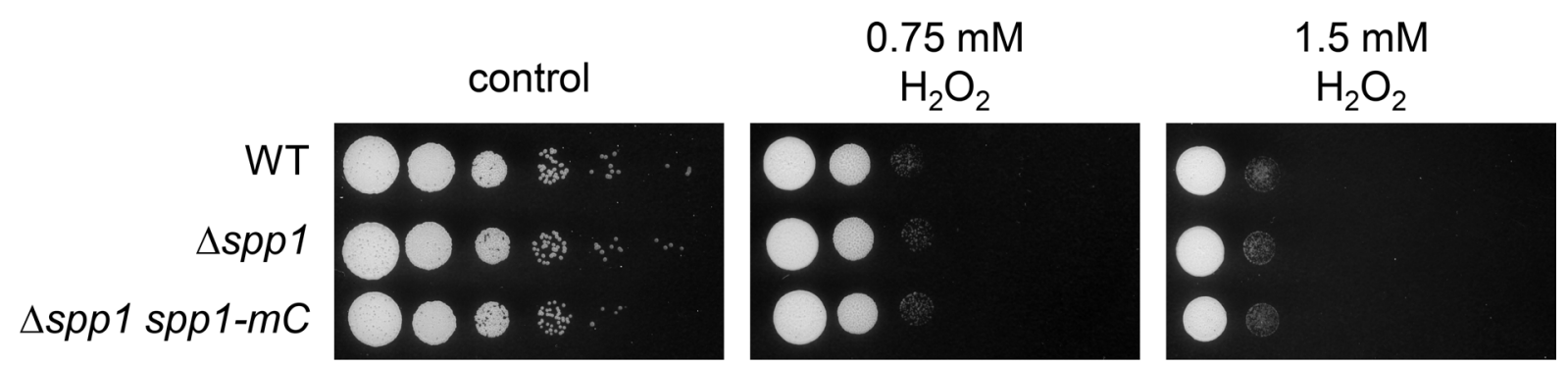

Figure 3.27: Spp1 is not crucial for $\mathrm{H}_{2} \mathrm{O}_{2}$ detoxification. $\mathrm{H}_{2} \mathrm{O}_{2}$ resistance of $U$. maydis strain SG200 (WT) and the $\Delta$ spp1 derivative was tested by serial 10-fold dilutions of strains, spotted on YNBG solid medium supplemented with the indicated concentration of $\mathrm{H}_{2} \mathrm{O}_{2}$. Plates were incubated for $48 \mathrm{~h}$ at $28^{\circ} \mathrm{C}$.

ROS are mainly derived from $\mathrm{H}_{2} \mathrm{O}_{2}$, which is produced by the NADPH oxidase complex in plants. (Bolwell and Wojtaszek, 1997). Therefore, the spp1 deletion strain was used in a plant infection assay with Diphenyleneiodonium (DPI), an inhibitor of ROS production mediated by the plant's NADPH oxidase. (Molina and Kahmann, 2007; Fernández-Alvarez et al., 2009). However, treatment with DPI did not complement the $\Delta$ spp1 phenotype (Fig 3.28A). Furthermore, in Chlorazol Black E stainings of infected leaf tissue, no difference between the treated (Fig 3.28B, $\Delta s p p 1$, DPI) and untreated (Fig 3.28B, $\Delta$ spp1, control) condition could be observed. 
A

B
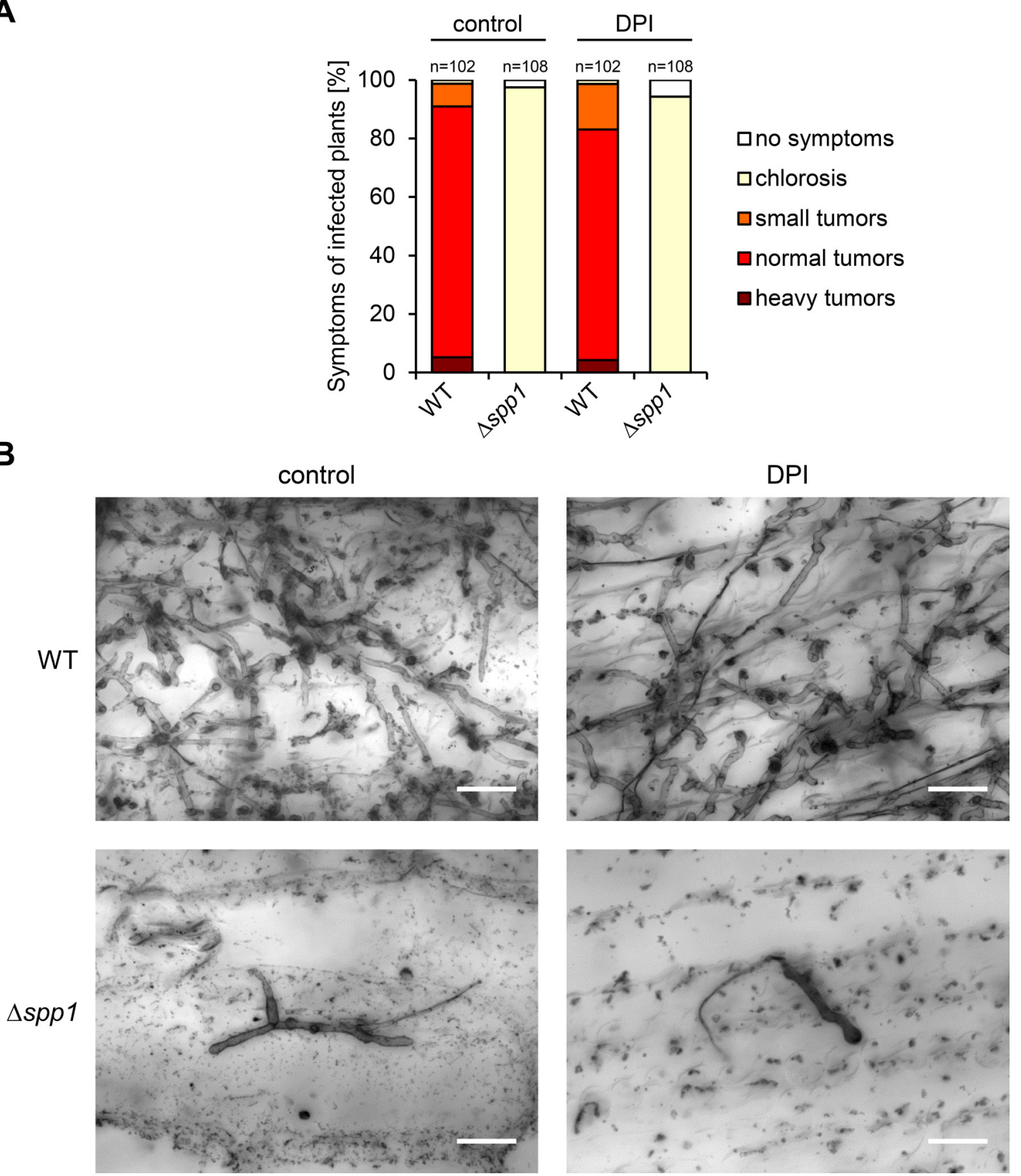

Figure 3.28: Inhibition of ROS production in planta could not recover the virulence of the $\Delta$ spp1 mutant.

(A) U. maydis strain SG200 (WT) and the $\Delta$ spp1 derivative were inoculated into 7-day-old maize seedlings. Cultures used for infection experiments were supplemented with $0.5 \mu \mathrm{M}$ (f.c.) DPI or an equivalent volume of solvent (DMSO). Disease symptoms were rated $8 \mathrm{dpi}$ and grouped into categories depicted on the right. $\mathrm{n}$ represents the total number of inoculated plants from three independent experiments. (B) Fungal morphology of SG200 (WT) and the $\Delta$ spp1 was investigated by Chlorazol Black E staining of DPI or control (DMSO) treated infected leaf samples at 3 dpi. Scale bar $=20 \mu \mathrm{m}$.

Since infection of maize plants with deletion strain of spp1 as well as the catalytic inactive spp $1^{\text {D279A }}$ mutant led to an increased plant defense response by increased ROS formation (Fig 3.26), gene expression of several plant defense response genes was tested in infected leaf tissue. To determine the gene expression of defense-related plant genes, the strains SG200 (WT), SG200 $\Delta$ spp1 ( $\Delta$ spp1) and

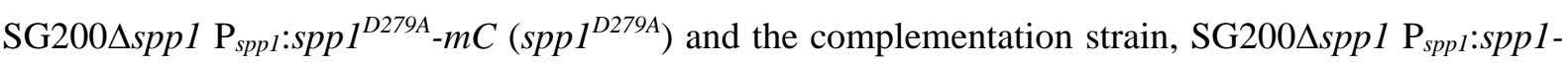
$m C$ ( $\Delta$ spp1 spp1), were cultivated in YEPS light to an $\mathrm{OD}_{600}$ of 1 and injected into 7-day-old maize 
seedlings. Infected leaf tissue was collected 2 dpi and expression plant genes was analyzed by qRTPCR. The tested genes can be divided into two groups: PR1, PR3, PR4, PR5, ATFP4 and POX12, which can be conflated in the group of salicylic acid (SA)-related defense response genes (Fig 3.29 and Appendix Fig 7.3, dark gray bars) and CC9 as well as BBI, which are allocated in the group of jasmonic acid (JA)-related response genes (Fig 3.29, light gray bars). Suppression of the plant defense responses by biotrophic pathogens is maintained by counteracting the SA response pathway and induce JA-related defense responses to prevent programmed plant cell death (Glazebrook, 2005). Consistently, all SA marker genes were highly induced in the $\Delta$ spp 1 and the $s p p 1^{\text {D279A }}$ strain compared to wildtype or the complementation strain (Fig 3.29 and Appendix Fig 7.3).
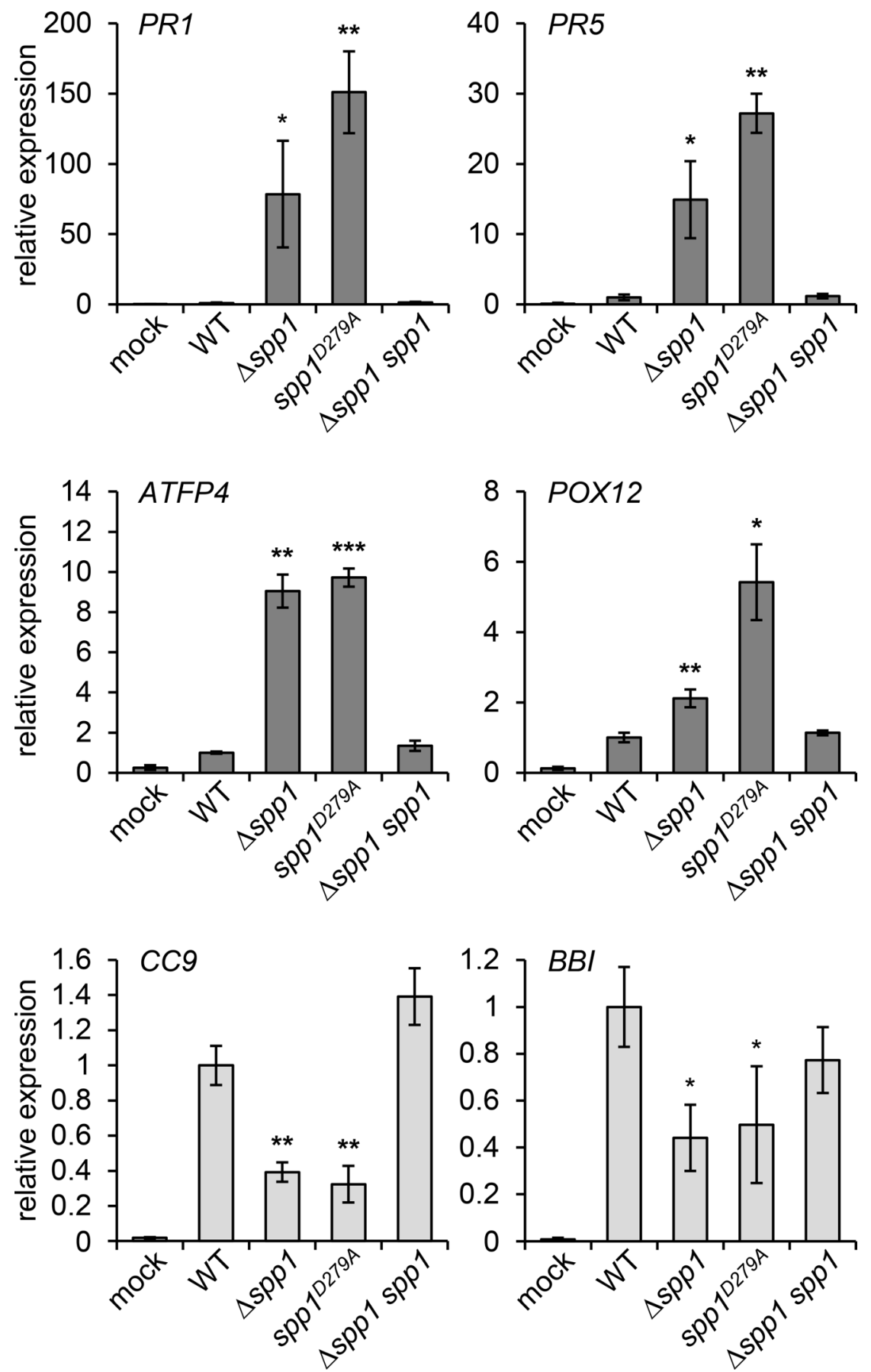

Figure 3.29: Strains with loss of the Spp1 function elicited strong induction of plant defense response genes during infection of Zea mays. qRT-PCR based expression analysis of defense-related $Z$. mays genes in response to infection with indicated U. maydis strains. 7-day-old maize seedlings were used for inoculation and samples of infected leaf tissue were collected 2 dpi. Expression levels are depicted relative to plants infected with the WT and represent the mean of three biological replicates with two technical duplicates each. GAPDH was used for normalization. Dark gray and light gray color of the bars indicate SA responsive genes (PR1, PR5, ATFP4, POX12) and JA responsive genes (CC9, $B B I$ ), respectively. Error bars represent the SD. Statistical significance was calculated using Student's $t$-test. *P-value $\leq 0.05, * * \mathrm{P}$-value $\leq 0.01$ and $* * *$ P-value $\leq 0.001$. Data partially generated in (Hach, 2018). 
In comparison to plants infected with the WT strain, $P R 1$ revealed the highest induction of all tested plant defense response genes. Gene expression of plants infected with the $\Delta s p p 1$ mutant or the spp $1^{\text {D279A }}$ mutant, had 78 mean fold changes (mfc) and $151 \mathrm{mfc}$, respectively, compared to plants infected with the wildtype strain. Differences in expression levels for PR5 compared with plants infected with the wildtype were $15 \mathrm{mfc}$ and $27 \mathrm{mfc}$ in plants infected with the spp1 deletion mutant or the catalytically inactive spp1 mutant, respectively. For ATFP4, a mean fold change of 9 for plants infected with the spp1 deletion mutant, as well as 10 for plants infected with the catalytically inactive spp1 was measured compared to plants infected with the wildtype. For the POX12 gene, $2 \mathrm{mfc}$ and $5 \mathrm{mfc}$ in expression levels compared to plants infected with the wildtype strain were measured for the spp1 deletion mutant and the catalytically inactive spp1 mutant, respectively (Fig 3.29).

For the additionally tested pathogenesis-related gene $P R 3$, a mean fold change of $2 \mathrm{mfc}$ and $4 \mathrm{mfc}$, for $\triangle$ spp1 and spp $1^{\mathrm{D} 279 A}$ were measured compared to the wildtype strain, and for PR4, mean fold changes of $14 \mathrm{mfc}$ and $24 \mathrm{mfc}$ for $\Delta s p p 1$ and spp $1^{D 279 A}$ were observed compared to the wildtype strain (Appendix Fig 7.3).

In contrast to the group of tested SA-responsive genes, the JA-responsive genes had an overall lower expression in planta. A decreased expression compared to the wildtype strain for CC9 could be observed with $3 \mathrm{mfc}$ for both, the spp1 deletion mutant as well as the catalytically inactive spp1 mutant. A decrease in the expression could also be observed for $B B I$ with $2 \mathrm{mfc}$ in the spp1 deletion mutant and the catalytically inactive spp1 mutant compared to the wildtype strain (Fig 3.29). Taken together, this indicates that the loss of the Spp1 function may be involved in the suppression of the plant defense responses.

\subsubsection{Deletion mutants of ER-associated degradation pathway (ERAD) and sterol biosynthesis regulator, Srb1, are not impaired in virulence}

The unfolded protein response is strongly interconnected to the ER-associated degradation pathway (Travers et al., 2000). Signal peptide peptidase activity is necessary for proper function of the ERAD in human cells (Chen et al., 2014). In S. cerevisiae, misfolded proteins in the ER are labeled for proteasomal degradation by three subpathways within ERAD: degradation of misfolded proteins at the cytoplasmic side (ERAD-C), degraded via the Doa10 E3 ligase complex, detection of misfolded proteins within the ER lumen (ERAD-L) or the ER membrane (ERAD-M) is mediated by the Hrd1 E3 ligase complex (Ruggiano et al., 2014). To examine, if the Spp1 function is connected to ERAD, gene deletions of ERAD components were generated and used in a plant infection assay. The main components of the Doa10 complex (Doa10) and the Hrd1 complex (Hrd1 and Der1) were identified in the U. maydis genome by a BLASTp analysis. Deletion mutants in the strain background SG200 of the genes hrd1 (UMAG_00542), der1 (UMAG_05898), doa10 (UMAG_10911) and der2 (UMAG_11402) were 
generated and tested in a plant infection assay to assess their role in pathogenicity. The strains were cultivated in YEPS light $_{\text {to }}$ an $\mathrm{OD}_{600}$ of 1 and injected to 7-day-old maize seedlings. None of the ERAD deletion mutants showed a reduced virulence compared to the wildtype strain. Surprisingly, neither the double deletion ( $\Delta h r d 1 \Delta$ doa 10$)$ nor the triple deletion strains $(\Delta h r d 1 \Delta$ doa10 $\Delta$ der1 and $\Delta h r d 1 \Delta$ doa10 $\Delta$ der2) were reduced in virulence, indicating that ERAD is not essential for pathogenicity in $U$. maydis (Fig 3.30). In addition, the strains were tested in an ER stress assay, by growing the cells in YEPS light $_{\text {to }}$ an $\mathrm{OD}_{600}$ of 1 and spotting them on YNBG solid medium containing TM. However, none of the strains showed an impairment in growth during extended UPR activity (Fig 3.31), indicating that the Spp1 function is not related to ERAD.
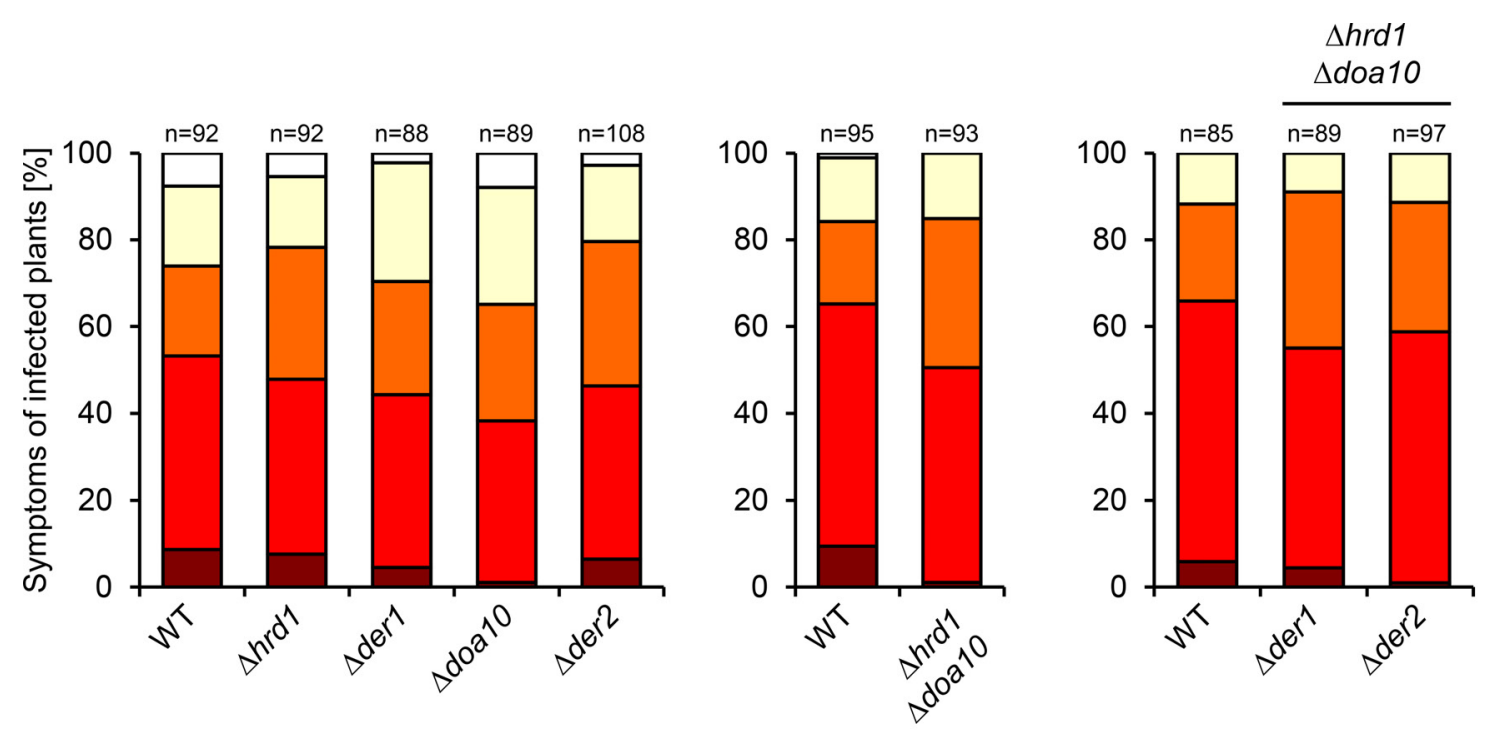

heavy tumors $\square$ normal tumors

small tumors

chlorosis

no symptoms

Figure 3.30: ERAD is dispensable for pathogenicity of $\boldsymbol{U}$. maydis. U. maydis strain SG200 (WT) and derivatives were inoculated into 7-day-old maize seedlings. Disease symptoms were rated 8 dpi. $n$ represents the total number of inoculated plants from three independent experiments. 


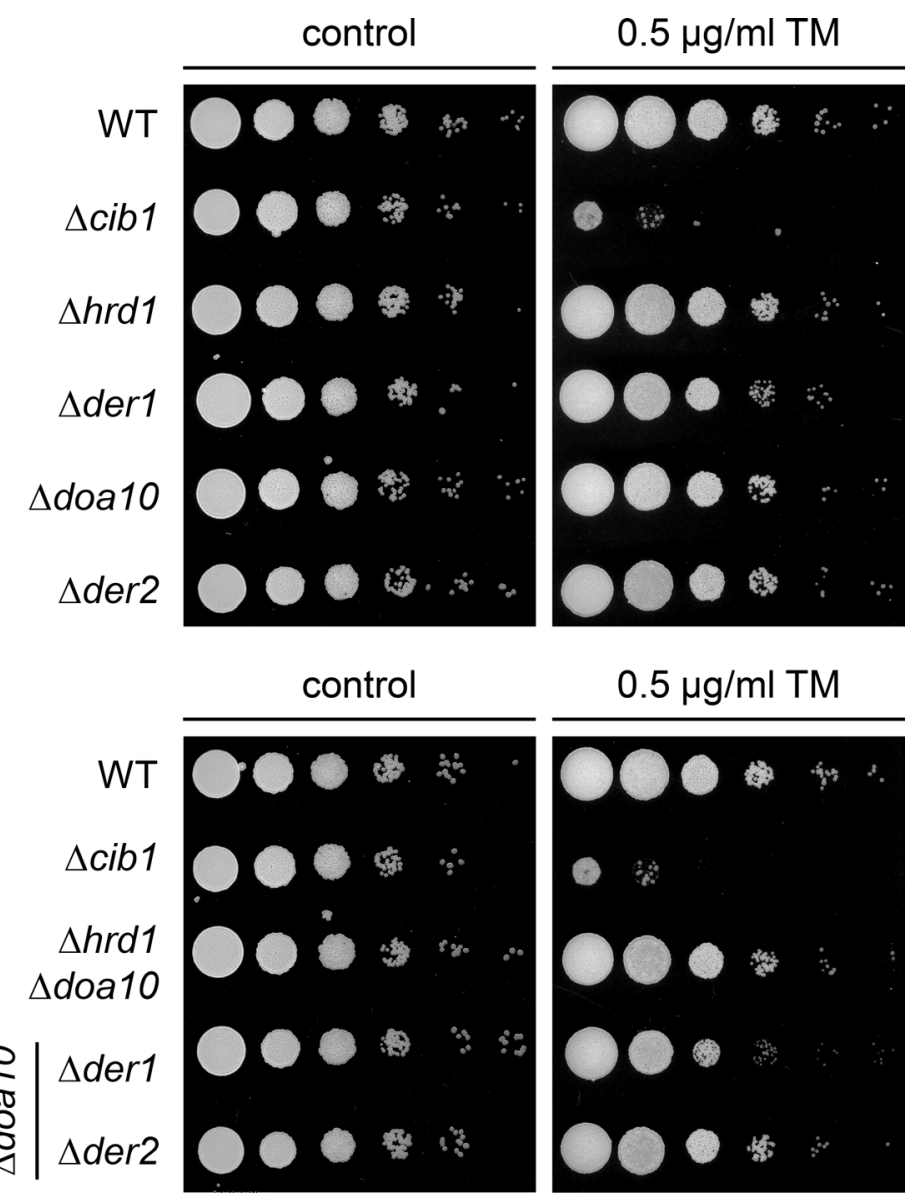

Figure 3.31: Deletion of ERAD genes did not affect ER stress tolerance. ER stress assay of $U$. maydis strain SG200 (WT) and derivatives. Serial 10-fold dilutions were spotted on YNBG solid medium supplemented with $\mathrm{TM}(0.5 \mu \mathrm{g} / \mathrm{ml})$ as indicated. Plates were incubated for $48 \mathrm{~h}$ at $28^{\circ} \mathrm{C}$.

In A. nidulans the signal peptide peptidase SppA was reported to be involved in cleavage and activation of the sterol regulatory element-binding protein (SREBP), SrbA, which is necessary for hypoxia adaptation (Bat-Ochir et al., 2016). SppA is highly similar to U. maydis Spp1 (E-value $8 \times 10^{-38}$ ) in the multiple alignment (Fig 3.19), which suggests a potential role of Spp1 in hypoxia adaption and cleavage of the identified srbA ortholog, srb1 (UMAG_05721), in U. maydis. A deletion mutant of srb1 was generated and tested in a plant infection assay, to examine if $s r b 1$ is involved in pathogenicity. The strain SG200 (WT) and srb1 deletion mutant ( $\Delta$ srb1) were grown in YEPS light to an OD $_{600}$ of 1 and inoculated into 7-day-old maize seedlings. However, the deletion mutant of $s r b 1$ does not affect pathogenicity compared to wildtype strain (Fig 3.32A). Moreover, the srb1 deletion strains were tested in an ER stress assay. The $\Delta s r b 1$ strain ( $\Delta s r b 1)$ did not show an impairment in growth during ER stress compared to the wildtype (Fig 3.32B). 
A

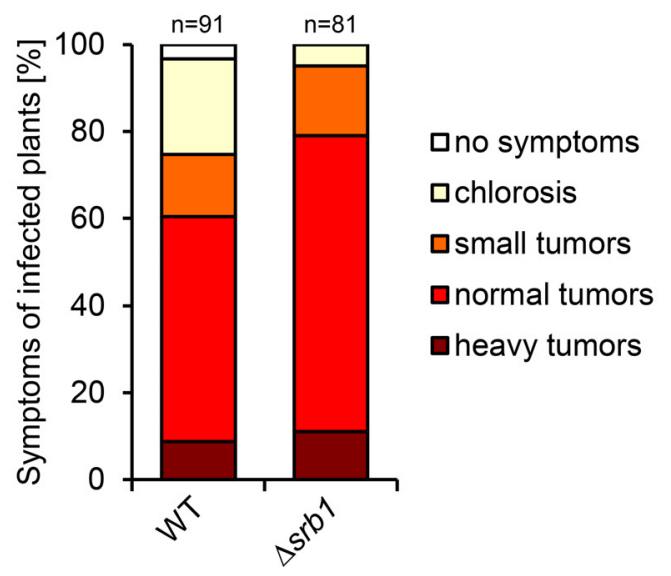

B

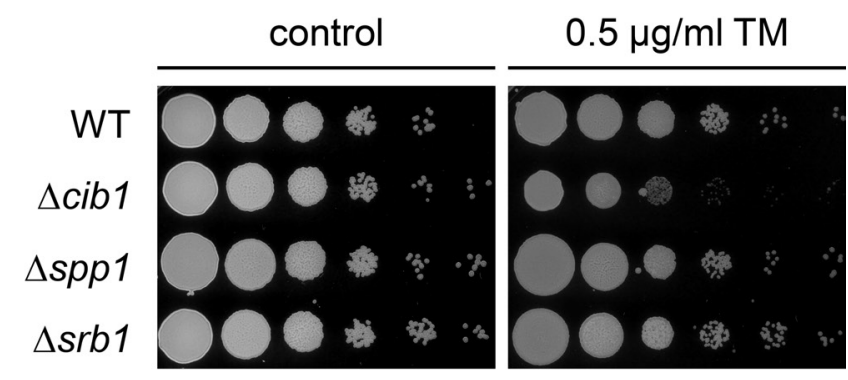

Figure 3.32: Characterization of the srb1 deletion strain in U. maydis. (A) U. maydis strain SG200 (WT) and the $\Delta s r b 1$ derivative were inoculated into 7-day-old maize seedlings. Disease symptoms were rated 8 dpi and grouped into categories depicted on the right. $\mathrm{n}$ represents the total number of inoculated plants from three independent experiments. (B) ER stress assay of $U$. maydis strain SG200 (WT) and the $\Delta$ srb1 derivative. Serial 10 -fold dilutions were spotted on YNBG solid medium supplemented with TM $(0.5 \mu \mathrm{g} / \mathrm{ml})$ as indicated. Plates were incubated for $48 \mathrm{~h}$ at $28^{\circ} \mathrm{C}$.

Furthermore, growth under hypoxic conditions was tested for the $\Delta$ spp1 mutant with additional UPR activation. However, no change in growth could be observed under hypoxia, neither with an inactive (Fig 3.33, TM, hypoxia) nor an active UPR (Fig 3.33, +TM, hypoxia). Both results indicate, that Spp1 is not involved in hypoxia adaption in U. maydis.

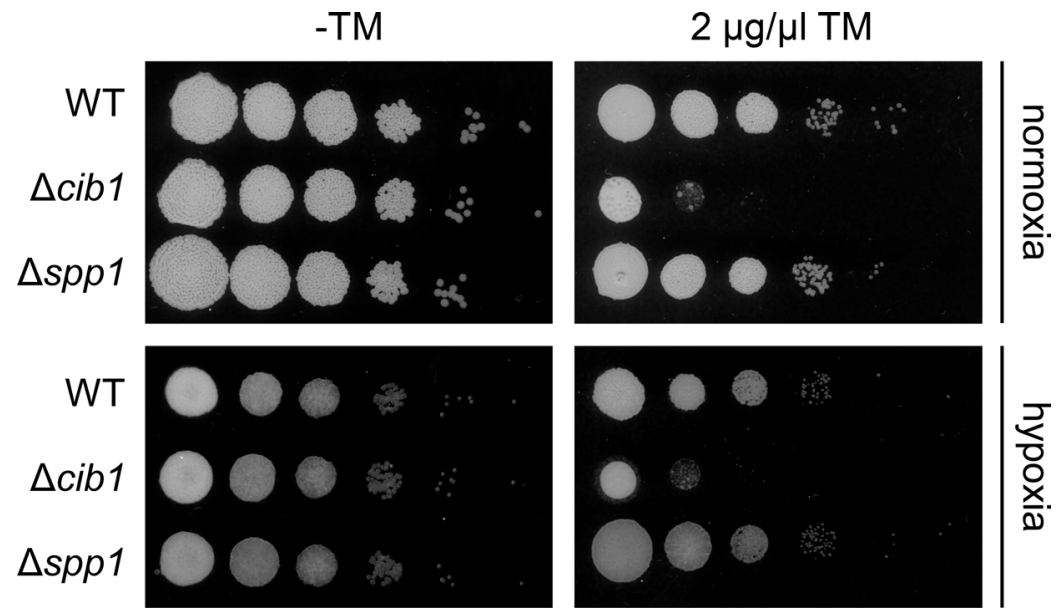

Figure 3.33: Growth of the $\triangle$ spp1 mutant is not impaired under hypoxic conditions. Hypoxia stress assay of U. maydis strain SG200 (WT) and derivatives. Serial 10-fold dilutions were spotted on YNBG solid medium supplemented with TM $(2 \mu \mathrm{g} / \mathrm{ml})$ as indicated. Plates were incubated in normoxia $\left(21 \% \mathrm{O}_{2}\right)$ and hypoxia $(1-5 \%$ $\mathrm{O}_{2}$ ) for $48 \mathrm{~h}$ at $28^{\circ} \mathrm{C}$. 


\subsubsection{Effector secretion is not affected in $\Delta$ spp1 strains}

Secretion of effector proteins during the biotrophic interaction of pathogens with their host is a crucial step in the infection process. In U. maydis, the UPR is required for efficient effector secretion (Hampel et al., 2016). To test if the loss of virulence of the $\Delta s p p 1$ mutant can be connected to altered protein secretion, a with the effectors pit2 (Hampel et al., 2016), pep1 (Doehlemann et al., 2009), tin2 (Tanaka et al., 2014) and cmu1 (Djamei et al., 2011) was performed.

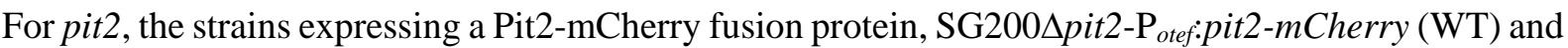

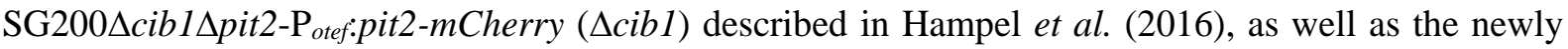

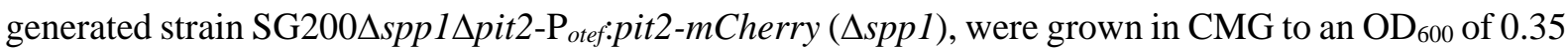
and treated (+) or untreated (-) with TM for 4 hours at $28^{\circ} \mathrm{C}$.
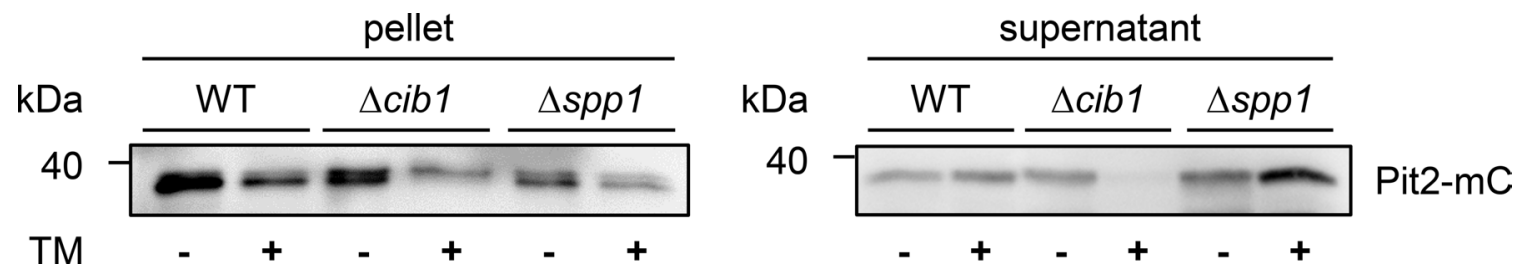

Figure 3.34: Secretion of Pit2-mC is not impaired in the spp1 deletion strain. Secretion of Pit2-mCherry was investigated by Western hybridization of protein extracts prepared from indicated strains expressing the Pit2mCherry fusion protein under the control of the constitutive active otef promoter. Strains were grown in CMG with or without $5 \mu \mathrm{g} / \mathrm{ml} \mathrm{TM} \mathrm{(+)}$ and were further incubated for $4 \mathrm{~h}$ at $28^{\circ} \mathrm{C}$. Cell pellets and supernatant were separated by centrifugation. Proteins were separated by SDS-PAGE analysis followed by Western hybridization using a mCherry specific antibody. Data generated in (Hach, 2018).

Secretion of Pit2-mC is strongly reduced under ER stress conditions in the deletion of the UPR regulator cib1 (Fig 3.34, supernatant, $\Delta c i b 1,+\mathrm{TM}$ ) compared to the wildtype (Fig 3.34, supernatant, WT, +TM). However, deletion of spp1 did not affect secretion of Pit2-mC under ER stress conditions (Fig 3.34, supernatant, $\Delta s p p 1+\mathrm{TM}$ ). A similar approach was used for the effectors pep1, tin2 and cmu1. The effectors were expressed under the constitutive active otef promoter as C-terminal mCherry fusion proteins in the wildtype (WT) as well as in the spp1 deletion ( $\Delta$ spp1) strain background. None of the tested effector proteins were affected in secretion by the spp1 deletion (Fig 3.35, supernatant, $\Delta$ spp1). UPR activation plays a major role in the post-translational modification of Pep1-mC and Cmu1-mC, resulting in a lower migrating protein band compared to the untreated condition in the WT and $\Delta$ spp1 strain background (Fig 3.35, pellet and supernatant, Pep1-mC and Cmu1-mC). However, UPR activation seems to be necessary for secretion of Pep1-mC and Tin2-mC, since only under ER stress secretion of either effector was observed (Fig 3.35, supernatant, Pep1-mC and Tin2-mC), which is not the case for Cmu1-mC, where a signal can be observed independently of ER stress (Fig 3.35, supernatant, Cmu1-mC). Taken together, the results of the secretion assays (Fig 3.34 and 3.35) suggest that deletion of spp1 is not required for effector secretion. However, secretion and processing of effector proteins in $U$. maydis seem to be closely connected to a functioning UPR. 


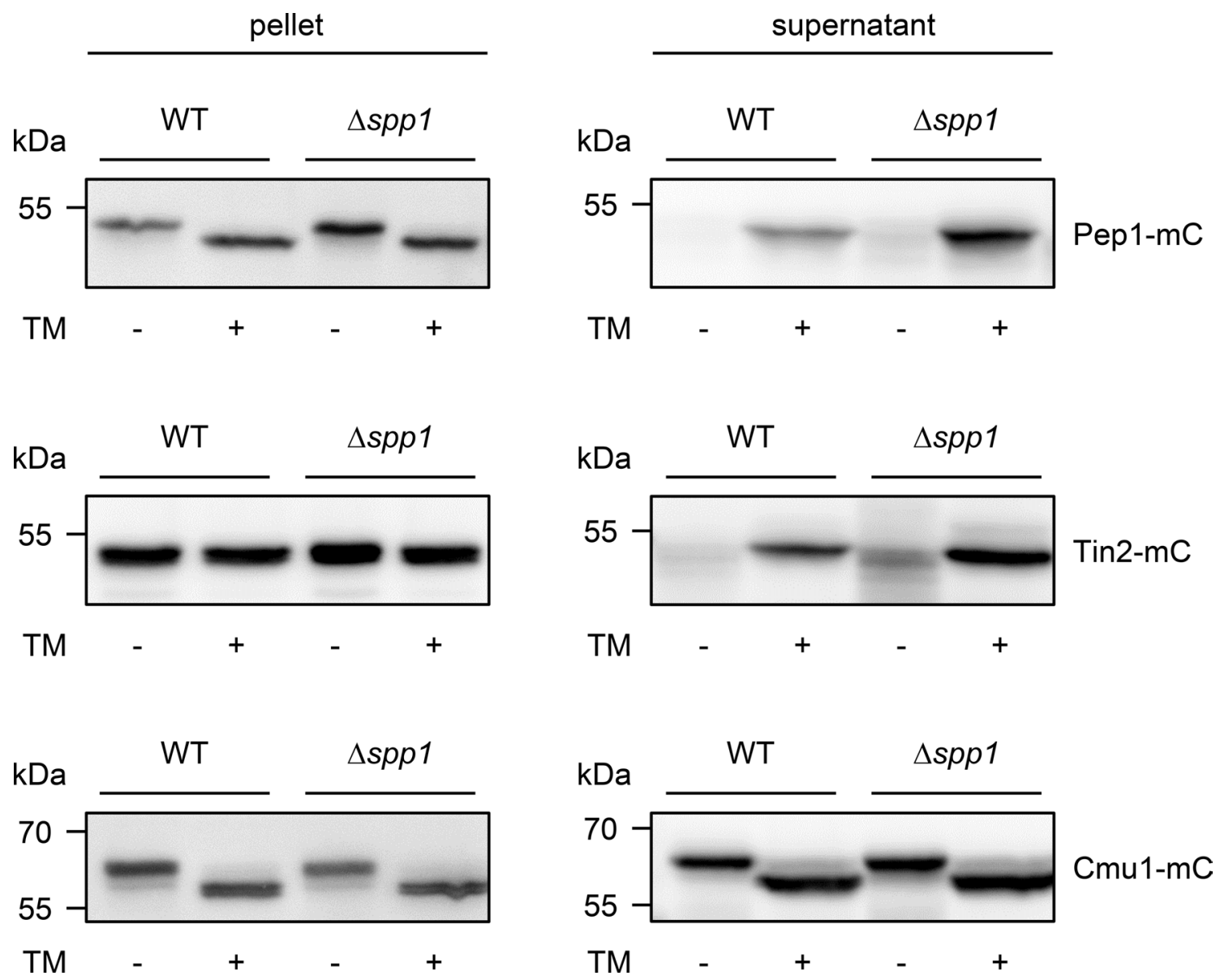

Figure 3.35: Secretion of Pep1-mC, Tin2-mC and Cmu1-mC is not impaired in the $\Delta$ spp1 strain. Effector secretion was investigated by Western hybridization of protein extracts prepared from indicated strains expressing the respective mCherry fusion proteins under the control of the constitutive active otef promoter. Strains were grown in CMG with or without $5 \mu \mathrm{g} / \mathrm{ml} \mathrm{TM}\left(^{+}\right)$and were further incubated for $4 \mathrm{~h}$ at $28^{\circ} \mathrm{C}$. Cell pellets and supernatant were separated by centrifugation. Proteins were separated by SDS-PAGE analysis followed by Western hybridization using a mCherry specific antibody.

\subsubsection{Fungal UPR in planta is not elevated in $\triangle$ spp1}

Secretion in $\Delta$ spp1 is not impaired under axenic conditions, as indicated by the performed secretion assays in chapter 3.3.8. However, it might be possible that Spp1 is necessary to cope with the high load on the ER during extended effector secretion in planta. To test this assumption, expression analysis of the fungal UPR maker genes cib1's bip1, Ihs1, cne1 and UMAG_11594 in plants infected with SG200

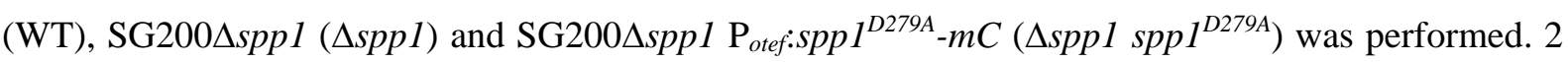
dpi, infected leaf tissue was collected and subjected to RNA preparation with subsequent qRT-PCR analysis. However, only UMAG_11594 showed a slight upregulation in both mutant strains. Fungal expression of other UPR marker genes was similar in the $\triangle$ spp1 and $\Delta$ spp1 spp1 ${ }^{\text {D279A }}$ strains compared to the wildtype (Fig 3.36) This indicates that the loss of virulence in $\Delta$ spp1 mutants is not due to an increased ER stress in planta, which would be predicted if secretion of effectors was affected. 


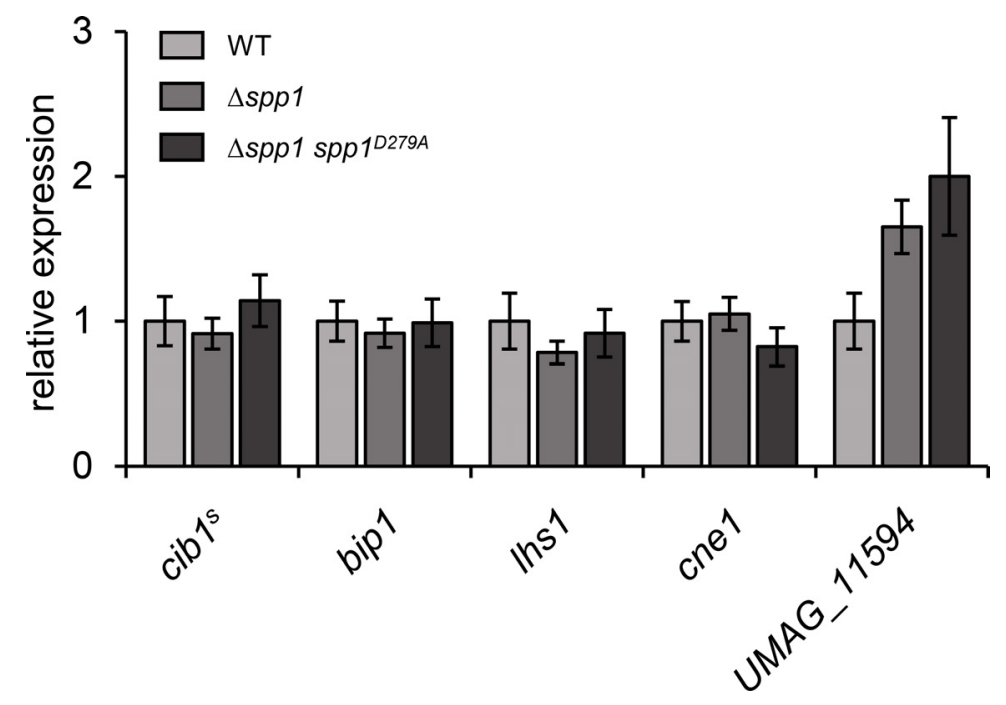

Figure 3.36: Genes of the fungal UPR are not upregulated in $\triangle$ spp1 strains during plant infection. qRT-PCR analysis was used to monitor fungal UPR gene expression in planta. Indicated $U$. maydis strains were inoculated in 7-day-old maize seedlings and infected leaf material was collected at 2 dpi. Expression levels are depicted relative to WT infected plants and represent the mean of three biological replicates with two technical duplicates each. eIF2b was used for normalization. Error bars represent the SD.

\subsubsection{Deletion of UPR elements (UPRE) in the spp1 promoter abolishes UPR-dependent induction of spp1}

Gene expression of spp1 is upregulated during the biotrophic interaction of $U$. maydis with its host plant and peak expression levels are reached 2 days after plant infection (Lanver et al., 2018). Moreover, RNAseq data and microscopic monitoring of Spp1 showed that the UPR regulator cib1 directly controls the gene expression of spp1 (Chapter 3.2.2 and 3.3.3). ChIPseq data of cib1 revealed a UPR element (UPRE), which consistently can be found in most of the UPR core gene promoters (Chapter 3.2.3). For spp1, two possible UPRE motifs, CTTCCACGTCT (Fig 3.37A and B, UPRE1) and GCGCTGTGCG (Fig 3.37A and B, UPRE2), were identified by their highest local peak enrichment in the spp1 promoter. 
A

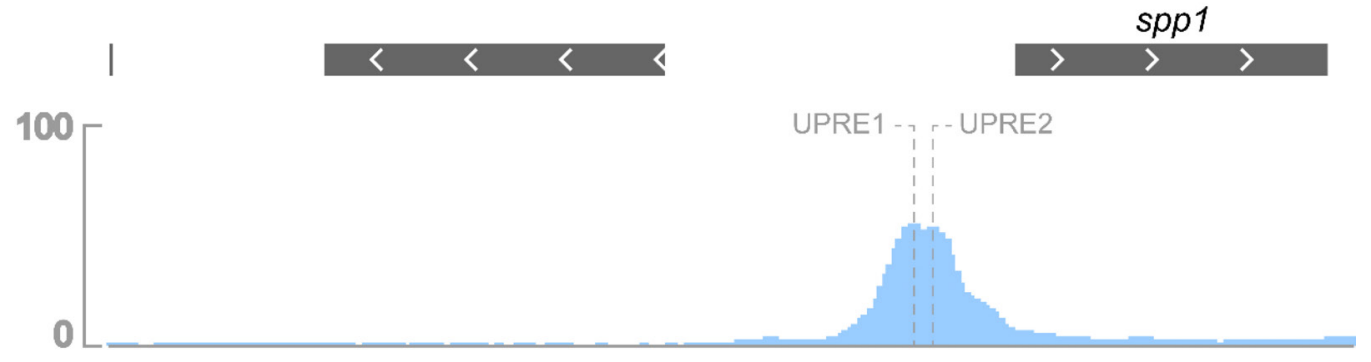

B

distance from tss motif
U. maydis UPRE1
$-433 \mathrm{bp}$
CTTCCACGTCT
U. maydis UPRE2
$-341 \mathrm{bp}$
GCGCTGTGCG

Figure 3.37: Scheme of UPREs in the spp1 promoter identified by ChIPseq. (A) Visualization of Cib1 binding in the spp1 promoter obtained by ChIPseq analysis (chapter 3.2.3). The depicted UPRE (UPRE1 and UPRE2) motifs were selected by their highest local peak enrichment (blue) in the promoter region of spp1. (B) List of identified UPREs in consecutive order. Nucleotides of UPREs are highlighted in their respective color. Distance from the spp1 transcription start site (tss) is depicted in base pairs (bp).

Both UPREs of the spp1 promoter were deleted, to check if the newly identified UPRE1 and UPRE2 in the spp1 promoter are important for expression of spp1 under ER stress. The strains, generated in the $\Delta$ spp1 deletion background, contained either a single deletion $\left(\mathrm{P}_{\text {spp1[AUPRE1]:spp1-mC or }}\right.$

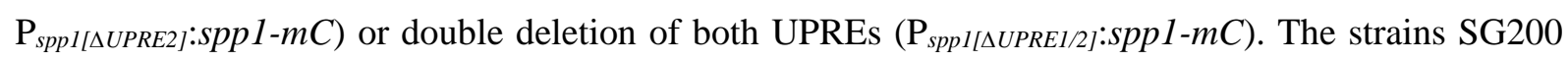

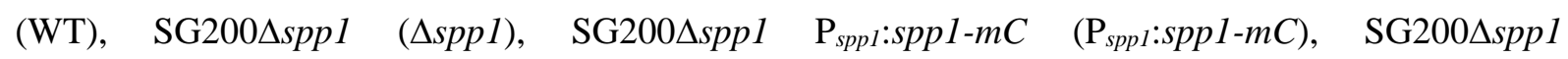

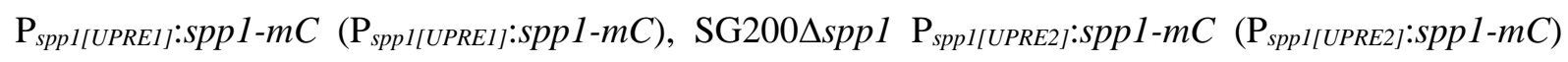

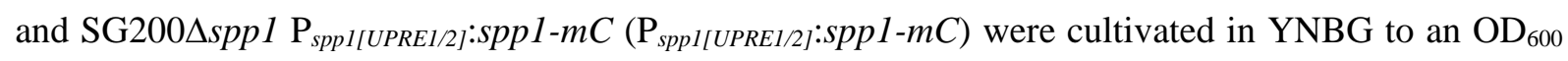
of 0.35 and treated with TM for 4 hours at $28^{\circ} \mathrm{C}$. Samples were collected and subjected to RNA preparation with subsequent qRT-PCR analysis. Induction of the UPR was tested by the UPR marker genes cib $1^{s}$ and bip1. Under unstressed conditions, expression of the UPR marker genes was similar in all strains. However, expression of spp1 in the untreated $\mathrm{P}_{\text {spp } 1}$ :spp1-mC was 4-fold increased when compared to wildtype strain (Fig 3.38, -TM). The expression of UPR marker genes during ER stress conditions was induced in all tested strains, whereas spp1 expression was only induced in the control strains but not in the UPRE deletion strains. 
$+\mathrm{TM}$

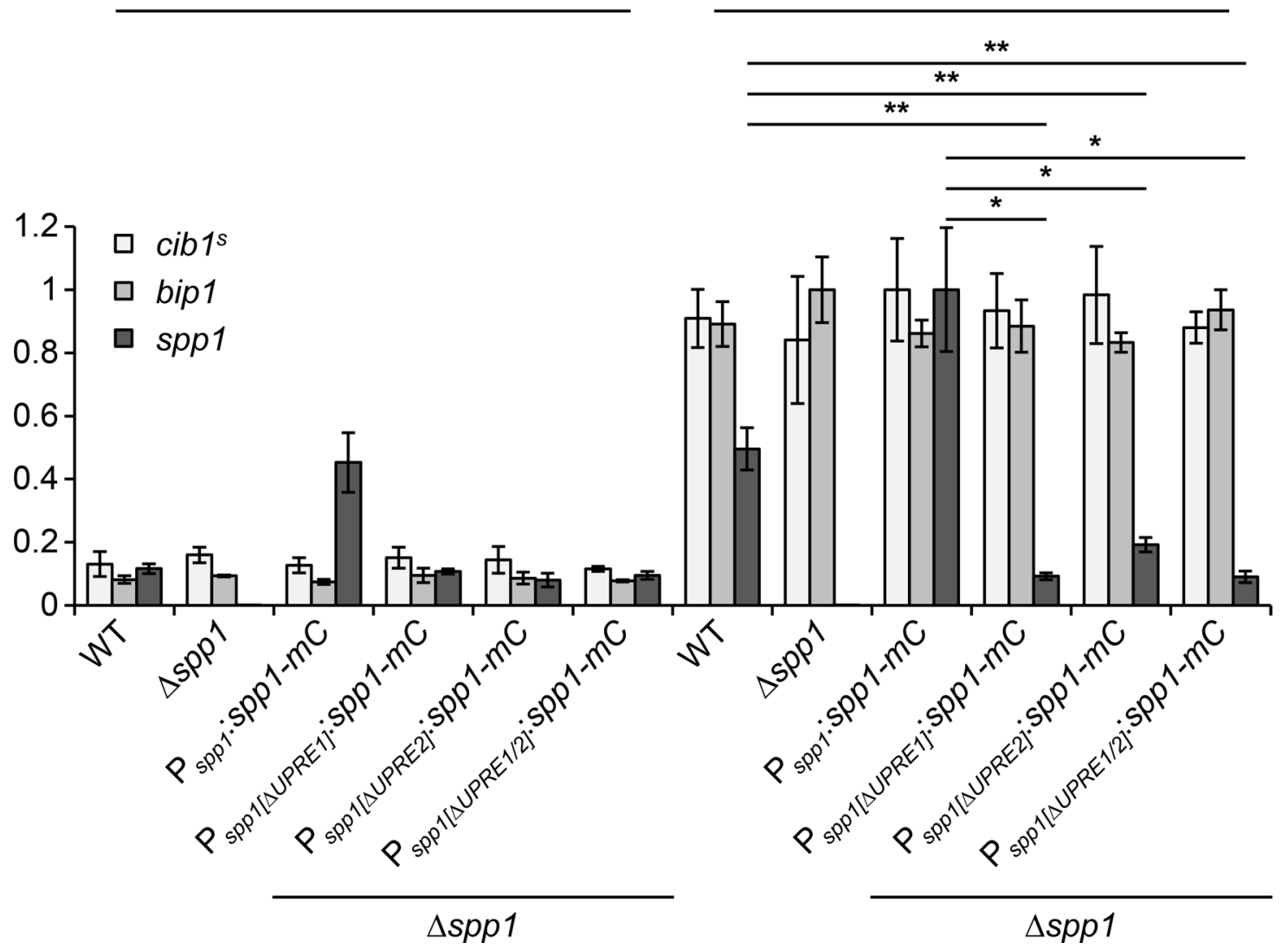

Figure 3.38: Strains with UPRE deletions in the spp1 promoter display a reduced expression during ER stress. qRT-PCR analysis was used to monitor spp1 gene expression in UPRE deletion strains. Indicated $U$. maydis strains were grown in YNBG to an $\mathrm{OD}_{600}$ of 0.35 and treated with or without $5 \mu \mathrm{g} / \mathrm{ml} \mathrm{TM}$ for $4 \mathrm{~h}$ at $28^{\circ} \mathrm{C}$. Expression levels are depicted relative to highest gene expression and represent the mean of three biological replicates with two technical duplicates each. eIF2b was used for normalization. Error bars represent the SD.

To examine, if UPRE dependent spp1 regulation is important for virulence, a plant infection assay was performed. The strains used in the qRT-PCR analysis (Fig 3.38) were grown in YEPS light $_{\text {to }}$ an $\mathrm{OD}_{600}$ of 1 and inoculate into 7-day-old maize seedlings. However, the UPRE deletion strains were still pathogenic but formed less heavy and more small tumors (Fig 3.39). These results indicate that the basal expression of spp1 in the UPRE deletion strains is still sufficient to establish a biotrophic interaction with the plant. 


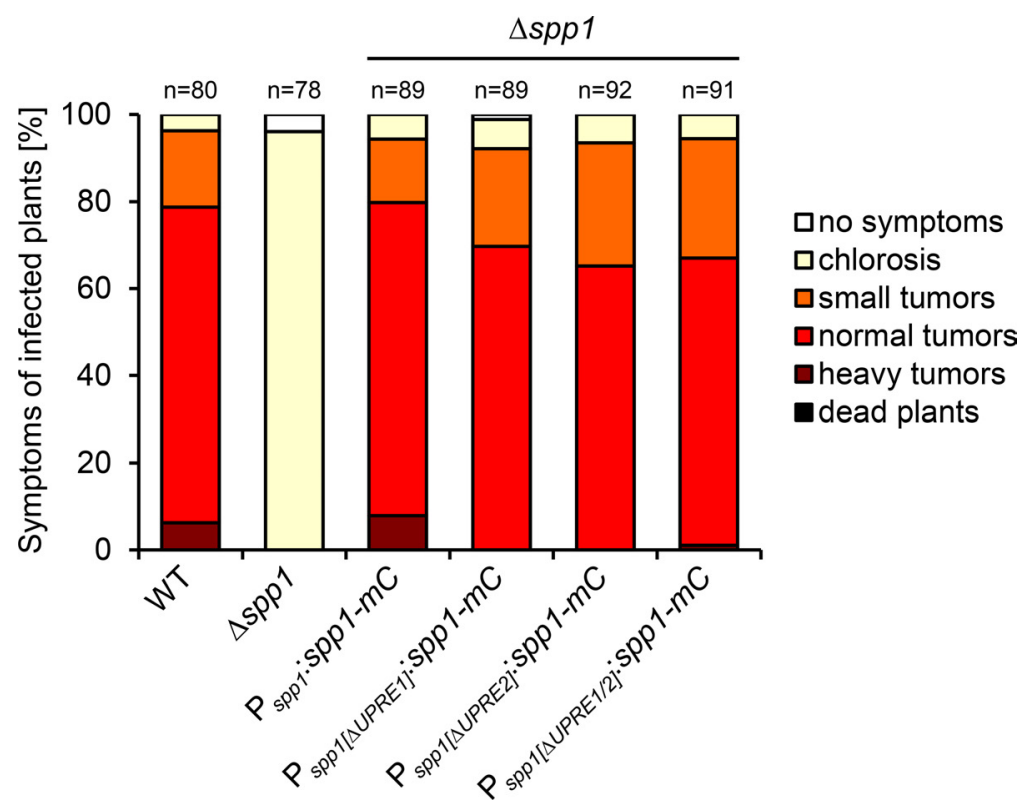

Figure 3.39: UPRE deletion mutants display a slightly reduced virulence. $U$. maydis strains SG200 (WT) and derivatives were inoculated into 7-day-old maize seedlings. Disease symptoms were rated 8 dpi and grouped into categories depicted on the right. $\mathrm{n}$ represents the total number of inoculated plants from two independent experiments.

\subsubsection{Bioinformatic prediction of UPR elements in SPP promoters}

Signal peptide peptidase activity in U. maydis is essential for pathogenicity (Chapter 3.3.1 and 3.3.5). Since the binding of Cib1 in the promoter region of spp1 is necessary for gene expression and a connection between the UPR and SPP expression is particular and not yet described, the question arises if the UPRE identified in the ChIPseq analysis (Chapter 3.2.3) can be found in other organisms SPP promoters. To answer this question, the identified UPRE (Fig 3.12) was subjected to MAST (Motif Alignment and Search Tool, (Bailey and Gribskov, 1998)) by using the SPP promoter regions of the smut fungi U. maydis (spp1), S. reilianum (Srspp1), U. hordei (Uhspp1) and U. bromivora (Ubspp1) for motif search (Fig 3.40 and 3.41).

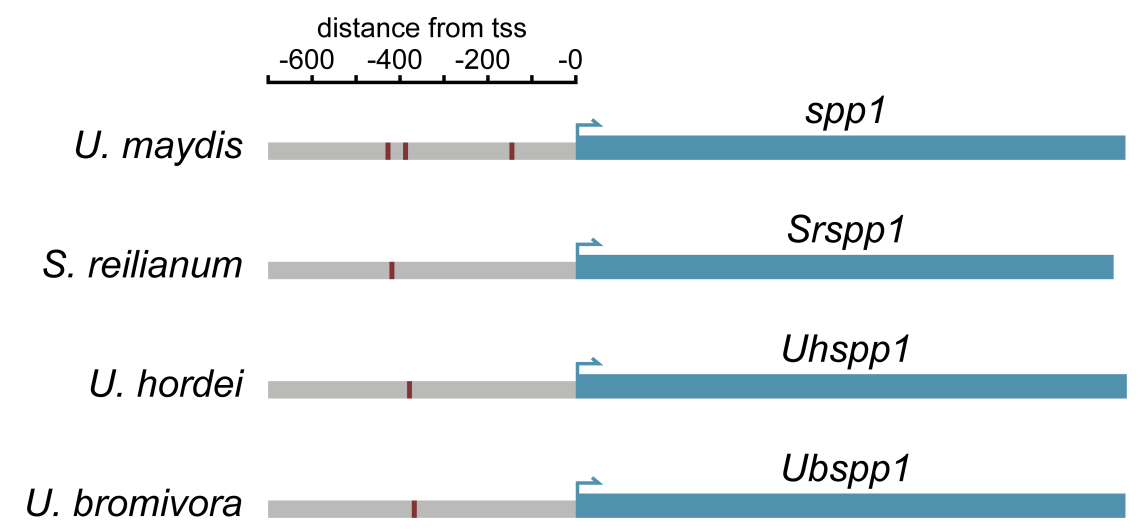

Figure 3.40: Schematic representation of predicted UPREs in promoters of spp1 and orthologous genes. The identified Cib1 binding motif of the WT strain was subjected to the MAST (ㅆotif $\underline{\text { Alignment and }}$ search $\underline{\text { Tool, }}$ http://meme-suite.org/tools/mast) for motif search in the SPP promoter region of U. maydis, S. reilianum, $U$. hordei and U. bromivora. Schematic representation of identified UPREs (red boxes). Promoter regions and genes are highlighted in gray and blue, respectively. Transcription start sites (tss) are indicated by arrows. 
The MAST analysis predicted two novel UPREs (predicted UPRE, pUPRE), pUPRE2

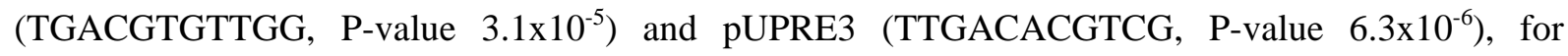
U. maydis spp1 promoter (Fig 3.41B). In addition, the predicted pUPRE1 (CTTCCACGTCT, P-value $6.3 \times 10^{-6}$ ) is similar to the identified UPRE1 in the UPRE deletion assay (Chapter 3.3.10). For S. reilianum, $U$. hordei and $U$. bromivora only one pUPRE could be identified in their SPP promoters,

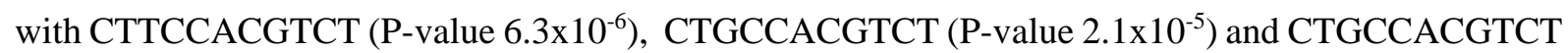
(P-value $2.1 \times 10^{-5}$ ), respectively (Fig 3.41). The results of the MAST analysis indicate that fungal virulence, promoted by Spp1, might not be solely restricted to U. maydis.

A

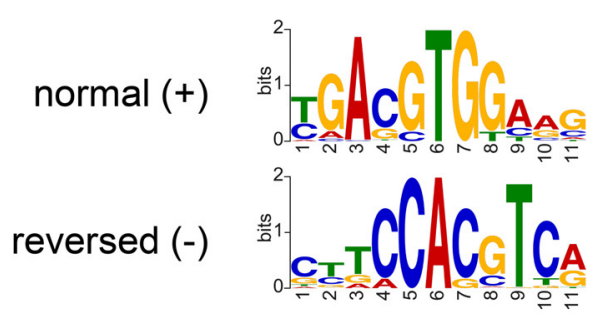

B

$\begin{array}{lcccc} & \text { distance from tss } & \text { motif } & \text { orientation } & \text { P-value } \\ \text { U. maydis pUPRE1 } & -433 \mathrm{bp} & \text { CTTCCACGTCT } & - & 6.3 \times 10^{-6} \\ \text { U. maydis pUPRE2 } & -393 \mathrm{bp} & \text { TGACGTGTTGG } & + & 3.1 \times 10^{-5} \\ \text { U. maydis pUPRE3 } & -151 \mathrm{bp} & \text { TTGACACGTCG } & - & 6.3 \times 10^{-6} \\ \text { S. reilianum pUPRE1 } & -424 \mathrm{bp} & \text { CTTCCACGTCT } & - & 6.3 \times 10^{-6} \\ \text { U. hordei pUPRE1 } & -384 \mathrm{bp} & \text { CTGCCACGTCT } & - & 2.1 \times 10^{-5} \\ \text { U. bromivora pUPRE1 } & -373 \mathrm{bp} & \text { CTGCCACGTCT } & - & 2.1 \times 10^{-5}\end{array}$

Figure 3.41: Predicted UPREs (pUPRE) in promoters of spp1 and orthologous genes. (A) The identified Cib1 binding motif of the WT described in figure 3.12 is depicted in both orientations. (B) List of identified UPREs in consecutive order. Nucleotides of UPREs are highlighted in their respective color. Distance from the SPP transcription start site (tss) is depicted in bp. P-value represents the probability of a single random subsequence of the length of the motif scoring at least as good as the observed match. Distance from the SPP transcription start site (tss) is depicted in base pairs (bp)

\subsubsection{LC-MS analysis identifies potential Spp1 interaction partners}

Besides the initially described function of signal peptide peptidases (SPP) in cleaving left-over signal peptides in the ER membrane, only a little is known about other potential substrates and the contribution of their cleavage products in cellular processes (Voss et al., 2013). Since Spp1 seems not to be involved in ERAD (Chapter 3.3.7), $\mathrm{H}_{2} \mathrm{O}_{2}$ detoxification (Chapter 3.3.6) and growth under hypoxia (Chapter 3.3.7), a co-immunoprecipitation assay with subsequent liquid chromatography-mass 
spectrometry (LC-MS) analysis was performed to identify possible interaction partners of Spp1 in U. maydis. To this end, strains were generated expressing the Spp1-GFP fusion protein and the catalytically inactive Spp1 ${ }^{\mathrm{D} 279 \mathrm{~A}}$-GFP mutant in the SG200Aspp1 background. Both fusion proteins were expressed under the control of the constitutive active otef promoter. The catalytically inactive Spp $1^{\mathrm{D} 279 \mathrm{~A}}$ mutant was used to inhibit substrate cleavage and by this increase the probability to identify putative protease/substrate interactions. These protein interactions are transient and often hard to detect using immunoprecipitation techniques. To identify unspecific protein binding to the GFP tag, the strain SG200

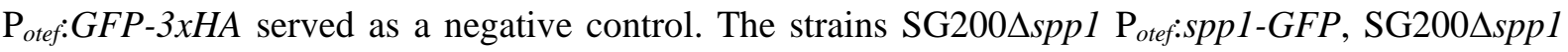
$\mathrm{P}_{\text {otef:Spp }} 1^{\text {D279A }}$-GFP and SG200 $\mathrm{P}_{\text {otef: }}$ GFP-3xHA were grown in CMG to an $\mathrm{OD}_{600}$ of 0.35 and treated 4 hours with or without TM for UPR induction. Protein extracts were subjected to immunoprecipitation using magnetic agarose GFP-trap beads. For quality control, an immunoblot was performed prior to LCMS analysis to check for sufficient protein levels in the untreated conditions, which was indeed the case (Fig 3.42).

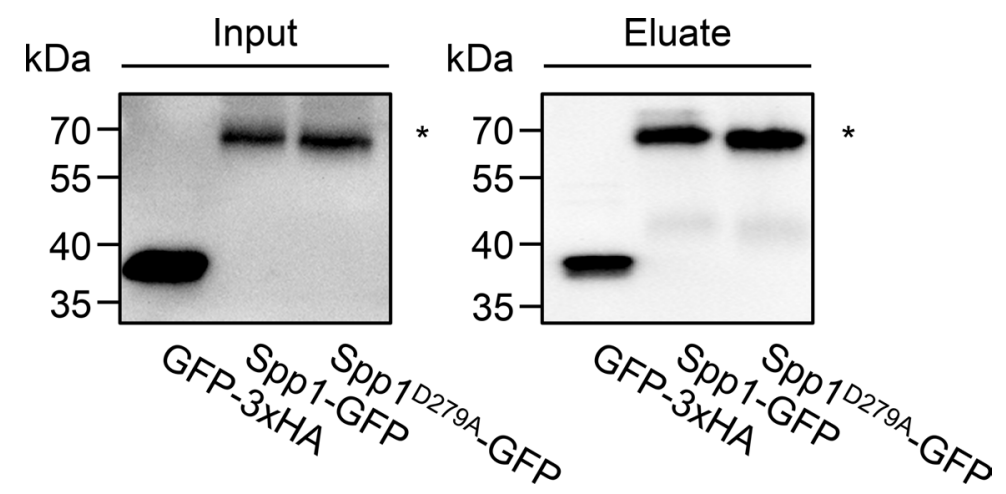

Figure 3.42: Western hybridization of pull-down of Spp1-GFP and Spp1 ${ }^{\text {D279A }}$-GFP. Input and eluate fractions of the untreated conditions were used for quality control prior to LC-MS analysis. Samples were resolved by SDSPAGE (10\%) and analyzed by Western hybridization. For detection of the Cib1-GFP fusion protein, a GFP specific antibody was used. The asterisk indicates the Spp1-GFP fusion protein with a predicted size of $\sim 73 \mathrm{kDa}$. Data generated in (Hach, 2018).

In the LC-MS analysis, a total number of 11 proteins were co-immunoprecipitated with Spp1-GFP and/or Spp1 ${ }^{\mathrm{D} 279 A}$-GFP. The proteins UMAG_02016 (Fig 3.43, not regulated in planta), UMAG_02266 (Fig 3.43, downregulated in planta), UMAG_04994 (Fig 3.43, upregulated in planta) and UMAG_06273 (Fig 3.43, not regulated in planta) were identified in the treated and untreated conditions of Spp1-GFP and Spp1 $1^{\mathrm{D} 279 \mathrm{~A}}$-GFP at least twice. A BLASTp analysis revealed that UMAG_02016 has a

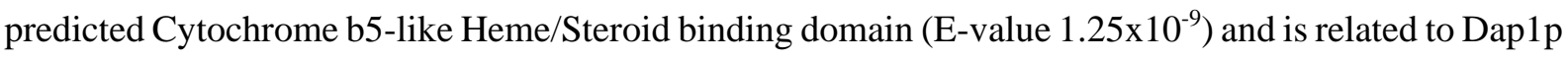
of S. cerevisiae (Similarity 66\%, E-value $4 \times 10^{-31}$ ). In Schizosaccharomyces pombe, Dap1 interacts with sterol biosynthesis proteins Erg5 and Erg11, which promotes normal sterol levels. The protein

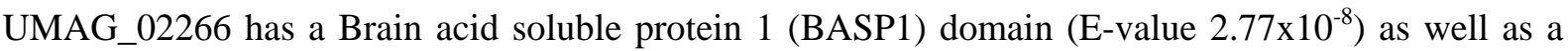
phosphatidate cytidylyltransferase domain (E-value $5.94 \times 10^{-121}$ ) and is related to Cds1p of S. cerevisiae (Similarity 52\%, E-value $8 \times 10^{-105}$ ), which is an ER membrane-bound CDP-diacylglycerol synthase essential for vegetative cell growth (Shen et al., 1996). The protein UMAG_04994 has an oligosaccharyltransferase subunit beta (48 kDa) domain (E-value $5.41 \times 10^{140}$ ) and is related to Wbp1p 
of S. cerevisiae (Similarity 47\%, E-value $7 \times 10^{-26}$ ). The protein UMAG_10649 is related to an oligosaccharyltransferase complex subunit delta (ribophorin II) (Similarity $44 \%$, E-value $1 \times 10^{-15}$ ) in Cryptococcus gattii. Members of the oligosaccharyltransferase (OST) protein complex are essential for N-linked glycosylation of proteins (Kelleher and Gilmore, 1994). The protein UMAG_06273 has a flavodoxin domain (E-value $1.37 \times 10^{-36}$ ) as well as an NADPH cytochrome p450 reductase (CYPOR) domain (E-value 0) and is related to Ncp1p of S. cerevisiae (Similarity 56\%, E-value $5 \times 10^{-152}$ ), which is involved sterol biosynthesis. The protein UMAG_02833 is related to the S. cerevisiae Sec66p (Similarity 55\%, E-value 2x10 ${ }^{-1}$ ) that is part of the Sec63 translocation complex (Feldheim et al., 1993). UMAG_05433 has a PMT1 domain (E-value 0) and is related to the dolichyl-phosphate-mannoseprotein O-mannosyl transferase Pmt4p of S. cerevisiae (Similarity 62\%, E-value 0) and is required for O-linked glycosylation of secretory and cell surface proteins (Sanders et al., 1999). The protein UMAG_11590 has a PqqL domain and is predicted to be a Zn-dependent peptidase (E-value 7.34x10${ }^{111}$ ). The protein is related to Mas1p of S. cerevisiae (Similarity $72 \%$, E-value $9 \times 10^{-170}$ ). Mas1p is a subunit of the mitochondrial processing protease (Witte et al., 1988). The protein UMAG_06480 does not have any conserved domains. Moreover, the protein can only be found in smut fungi such as U. bromivora (Similarity 78\%, E-value $7 \times 10^{-95}$ ), S. reilianum (Similarity $83 \%$, E-value $1 \times 10^{-85}$ ) and U. hordei (Similarity $80 \%$, E-value $8 \times 10^{-80}$ ).

The protein UMAG_06089 (Fig 3.43, upregulated in planta) was only present in the treated Spp1-GFP and Spp $1^{\text {D279A }}$-GFP conditions. BLASTp analysis of UMAG_06089 predicted a conserved microsomal signal peptidase $25 \mathrm{kDa}$ subunit (SPC25) domain (E-value $3.04 \times 10^{-38}$ ), which is part of the signal peptidase complex. The gene is a member of the UPR core genes (Fig 3.10), which is essential for U. maydis (Chapter 3.3.1)

The protein UMAG_02578 (Fig 3.43, not regulated in planta) was only detectable in conditions with the catalytically inactive Spp $1^{\text {D279A }}$-GFP mutant strain. In a BLASTp analysis of the protein sequence, a luminal heterokaryon incompatibility protein (Het-C) domain was predicted (E-value 0 ) that is related to het-C of Neurospora crassa (Similarity 53\%, E-value $4 \times 10^{-140}$ ) as well as TinC of A. nidulans (Similarity $60 \%$, E-value $4 \times 10^{-136}$ ). The protein in $U$. maydis has a predicted transmembrane domain (Phobius) and a larger cytosolic domain (241 AA), which is only present in smut fungi such as $U$. bromivora, S. reilianum and $U$. hordei. 


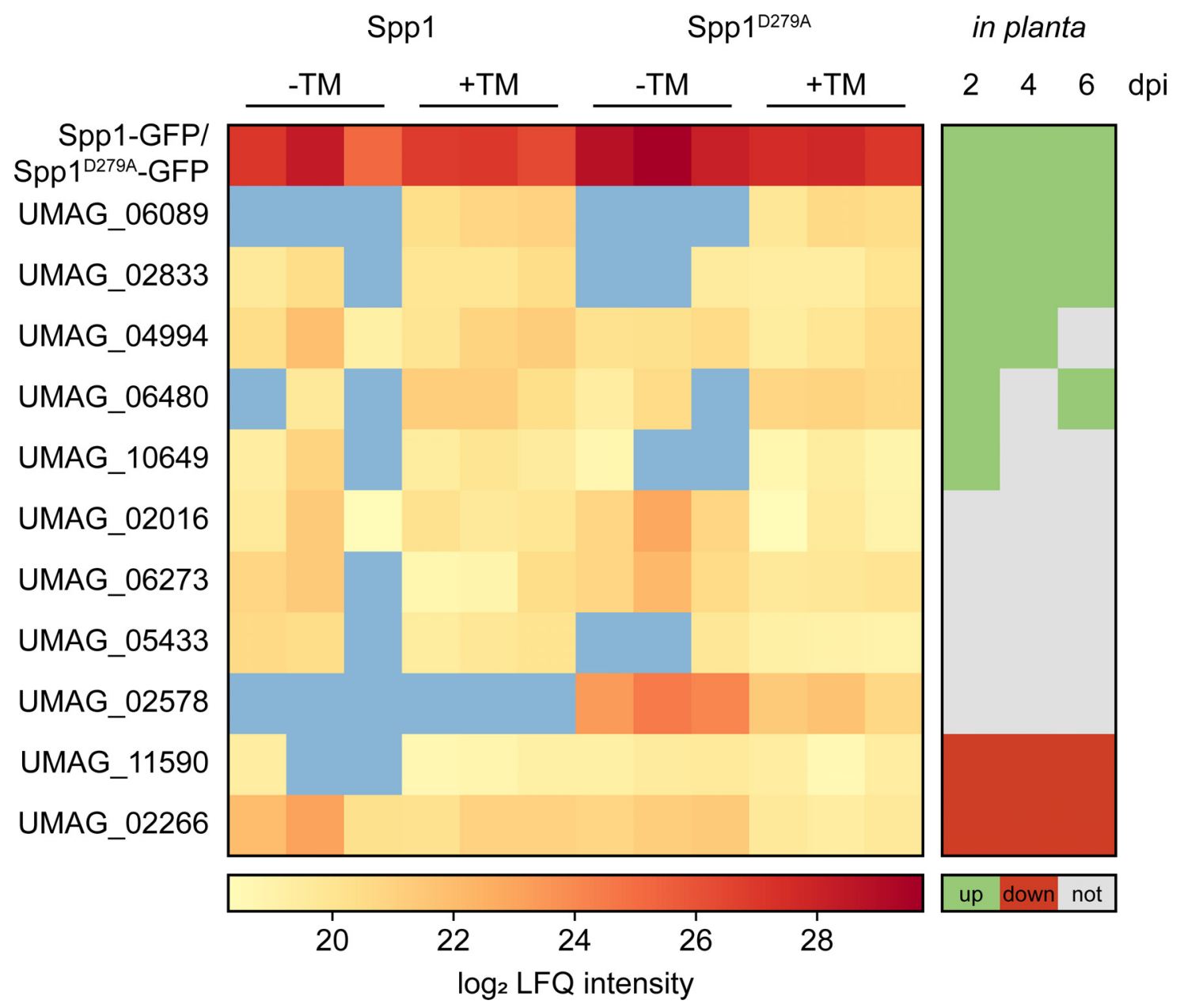

Figure 3.43: Heat map of potential Spp1 interaction partners identified by LC-MS analysis. For LC-MS

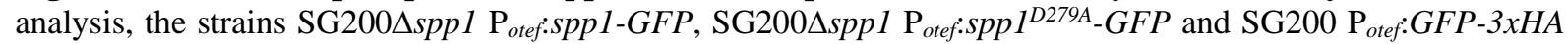
were grown in CMG to an $\mathrm{OD}_{600}$ of 0.35 and treated $4 \mathrm{~h}$ with or without TM (5 $\mu \mathrm{g} / \mathrm{ml}$ f.c.) for UPR induction. Samples were taken and prepared protein extracts were subjected for immunoprecipitation with magnetic agarose GFP-trap beads (Chromotek). Proteins were eluted and subjected to LC-MS analysis. Data analysis was performed with MaxQuant (https://maxquant.org) 1.6.0.16 (parameter file in Appendix File 2) using the label-free quantification method. For statistical analysis of the MaxQuant output, the Perseus (1.6.2.3) framework was used. The heat map was generated in Perseus by filtering towards proteins being detected in the treated Spp1 ${ }^{\mathrm{D} 279 \mathrm{~A}}-\mathrm{GFP}$ condition. The GFP-3xHA control was used as a negative control, to decrease the number of non-specific or noninteracting proteins. The LC-MS analysis was performed in three independent replicates, each indicated as a column in the heat map. Values for the proteins (rows) and the conditions (columns) are colored based on the protein abundance, in which high and low $\log _{2}$ LFQ (label-free quantification) intensity values are depicted in red and yellow, respectively. The range of $\log _{2}$ LFQ intensity values is indicated in the color bar shown below. Sample types (Spp1-GFP, Spp1 ${ }^{\mathrm{D} 279 \mathrm{~A}}-\mathrm{GFP}$ ) and treatment conditions (without tunicamycin [-TM], with tunicamycin [+TM]) are indicated at the top. Gene regulation of the identified proteins in planta (Lanver et al., 2018) is depicted for 2, 4 and 6 days after inoculation (dpi). Green, gray and red boxes indicate upregulation, the absence of differential gene expression and downregulation, respectively. Data generated in (Hach, 2018).

To examine if UMAG_02578 is involved in processes mediated by Spp1, subcellular localization was examined and deletions strains of UMAG_02578 were tested in a plant infection assay. UMAG_02578 was tagged with GFP and expressed under the constitutive active otef promoter to determine its subcellular location during unstressed and ER stress conditions. The strain was grown in CMG to an $\mathrm{OD}_{600}$ of 0.8 and treated with or without TM for 2 hours at $28^{\circ} \mathrm{C}$. The fusion protein UMAG_02578GFP is observable at ER-like structures and around the nucleus in treated and untreated conditions. 
However, after UPR activation, a more prominent signal in the nucleus can be observed (Fig 3.44, +TM, white arrows).

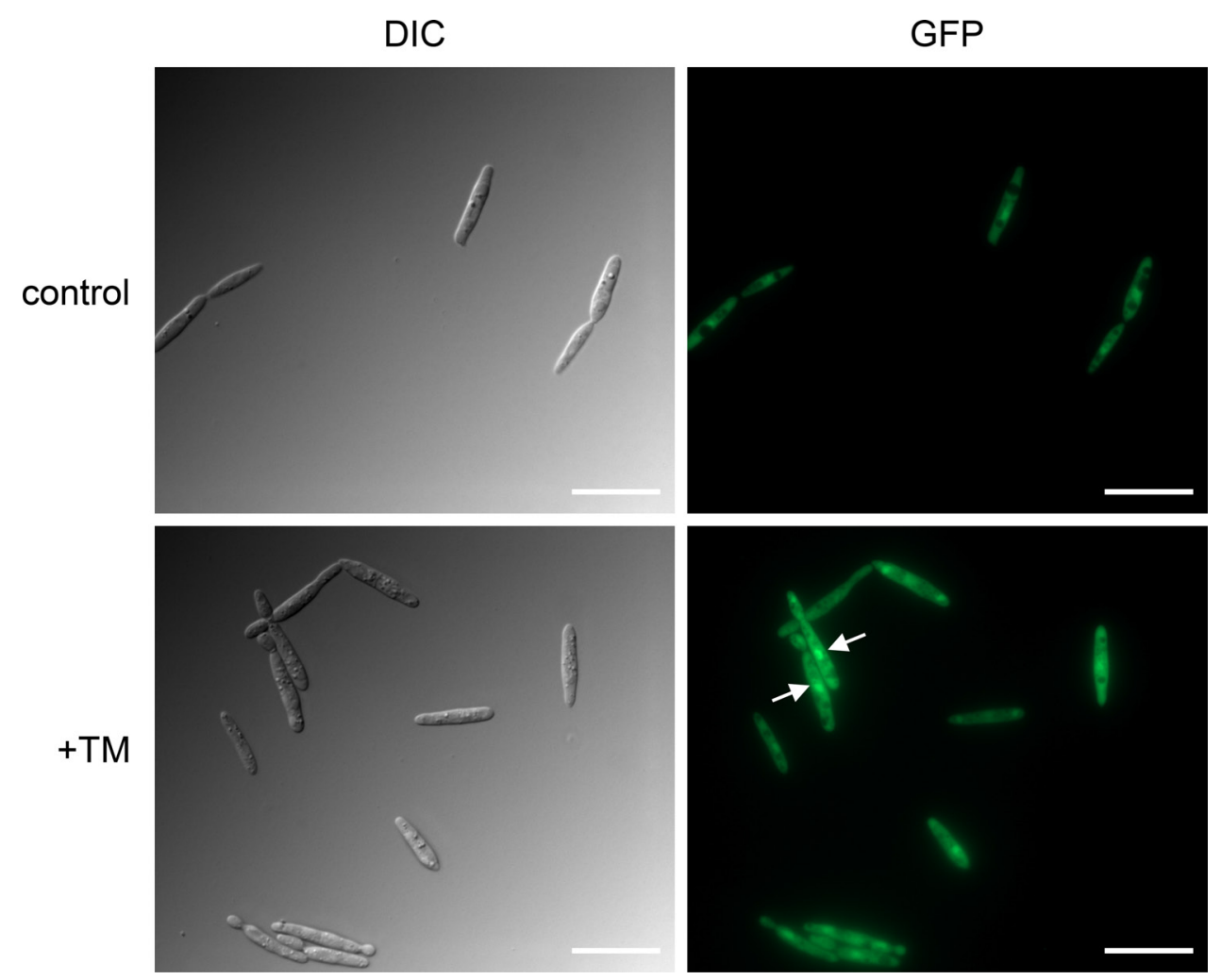

Figure 3.44: UMAG_02578-GFP accumulates upon ER stress at the nucleus of $\boldsymbol{U}$. maydis. The strain SG200

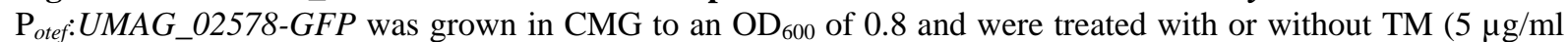
f.c.) for $2 \mathrm{~h}$ at $28^{\circ} \mathrm{C}$ to activate the UPR. DIC = differential interference contrast. Exposure time of GFP channel was set to $350 \mathrm{~ms}$. Scale bar $=20 \mu \mathrm{m}$. Data generated in (Hach, 2018).

An infection assay was performed to address the question if UMAG_02578 is essential for pathogenicity. To this end, deletion mutants of UMAG_02578 were generated. The strains SG200 (WT) and the derivatives $\triangle U M A G \_02578 \# 1, \Delta U M A G \_02578 \# 3$ and $\triangle U M A G \_02578 \# 5$ were grown in YEPS light to an $\mathrm{OD}_{600}$ of 1 and inoculated into 7-day-old maize seedlings. However, all of the UMAG_02578 deletion mutants are indistinguishable from the wildtype strain (Fig 3.45). The function of the protein interaction between Spp1 and UMAG_02578 remains unclear and may be elucidated in the future. 


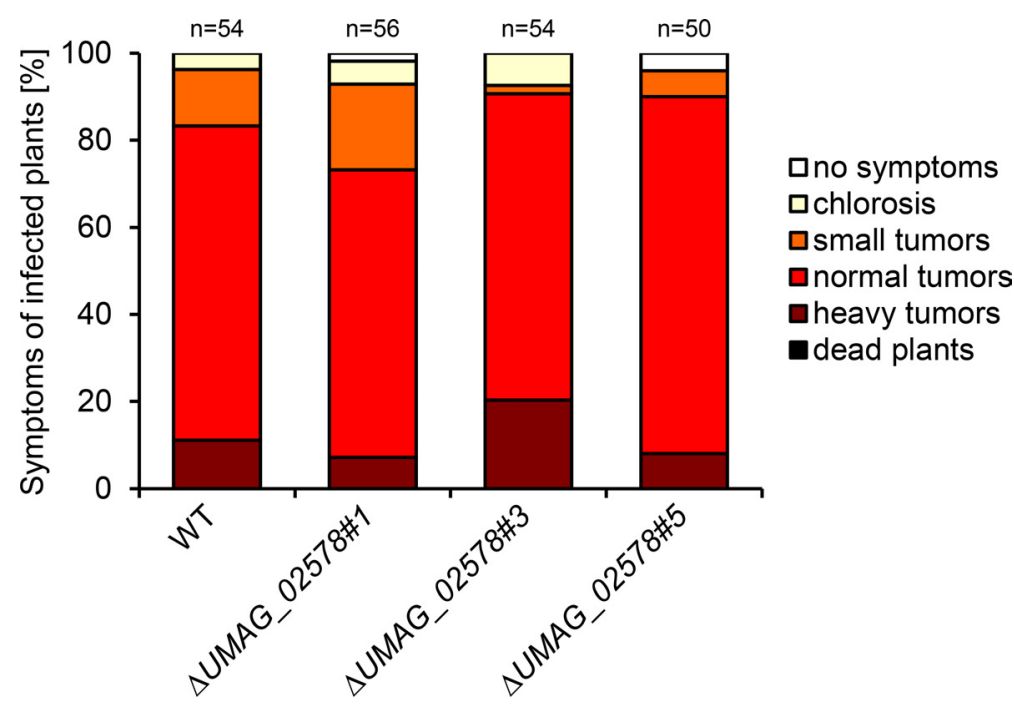

Figure 3.45: Deletion mutants of UMAG_02578 have no impact on pathogenicity. U. maydis strain SG200 (WT) and derivative were inoculated into 7-day-old maize seedlings. Disease symptoms were rated 8 dpi and grouped into categories depicted on the right. $\mathrm{n}$ represents the total number of inoculated plants. 


\section{Discussion}

In this study, the Clp1-dependent modulation of the UPR during biotrophic development of $U$. maydis was analyzed. It could be shown that the interaction of Clp1 with the central regulator of the UPR, Cib1, impacts subcellular localization, increases protein levels and stability, and alters phosphorylation pattern of Cib1. Further, it could be demonstrated, that the transcriptional output of the UPR upon clp1 expression modulates a set of UPR core genes identified by RNAseq analysis. The observed modulation of the UPR is in line with previous studies on the regulation of specific UPR target genes during Clp1 induction (Heimel et al., 2013). However, a genome-wide analysis to identify UPRE motifs in promoters of UPR regulated genes did not show an altered DNA binding of Cib1 during Clp1 expression. Deletion of UPR core genes that are not repressed upon Clp1 expression identified a novel pathogenicity factor spp1, encoding an ER resident signal peptide peptidase (SPP), specifically upregulated during pathogenic development (Lanver et al., 2018). Deletion of spp1 resulted in impaired growth in planta and a complete loss of virulence. Moreover, infected maize plants showed highly increased defense responses as evidenced by the production of reactive oxygen species and upregulation of defense-related genes. The virulence function of Spp1 is not connected to previously known SPP functions or cellular pathways in other organisms such as ER-associated degradation and hypoxia adaptation but requires Spp1 catalytic activity. This suggests that specific cleavage products generated by Spp1 catalytic activity, are of crucial importance to establish a compatible biotrophic fungal/plant interaction.

\subsection{Post-translational control of the UPR regulator Cib1}

In $U$. maydis, plant penetration marks a key step in the host adaptation of the fungus during biotrophic development. Further steps after the onset of plant penetration are controlled by the developmental regulator Clp1 and an active UPR (Heimel et al., 2010a; Heimel et al., 2013). The bZip transcription factor Cib1 is the central regulator of the UPR in U. maydis, and its interaction with Clp1 promotes in planta proliferation during pathogenic development (Heimel et al., 2013). Moreover, the interaction of Clp1 with Cib1 leads to an elevated ER stress resistance (Fig 3.1). Fluorescence microscopy of infected maize plants with strains expressing a Cib1-GFP fusion protein showed a strong nuclear fluorescence signal only after plant penetration (Heimel et al., 2010a). The Clp1 protein is highly unstable and UPR activation increases its stability significantly by physical interaction with Cib1 (Heimel et al., 2013). However, the consequences of Clp1 expression on the Cib1 protein have not been addressed, yet.

\subsubsection{Cib1 mutual stabilize each other Clp1 upon interaction}

To investigate the impact of Clp1 interaction with Cib1, Cib1 protein and cib1 $1^{s}$ transcript levels were monitored during clp1 expression (Fig 3.3). Protein levels of Cib1 were strongly increased in clp1 expressing conditions (Fig 3.3A) and as reported before (Heimel et al., 2013), expression levels of $c i b 1^{s}$ 
were significantly lower in $c l p 1$ expressing conditions (Fig 3.3B). This opens the question of whether increased stability of Cib1 or enhanced translation of the $\operatorname{cib} 1^{s}$ mRNA led to elevated Cib1 protein levels. Stability of Cib1 during clp1-expression was monitored over time with a cycloheximide chase assay (Fig 3.4A) and a doxycycline promoter shut-off assay (Fig 3.4B). Both assays showed a significant increase over time in Cib1 protein stability during the co-expression of Clp1. According to the promoter shut-off assay (Fig 3.4A), the estimated protein half-life of Cib1-GFP fusion protein increased from $2 \mathrm{~h}$ to more than $3 \mathrm{~h}$. In line with this observation, it could be shown that in human cancer cell lines, a mutual increase of protein half-life was shown for the bHLH-type transcription factor STRA13 and its interactor MSP58, by possibly preventing proteasomal degradation (Ivanova et al., 2005). The observation of recent studies (Heimel et al., 2013), that increased stability of Clp1 is promoted by physical Cib1 interaction is further supported by the performed stability assays (Fig 3.4A and B) of the present study, indicating a positive, mutualistic effect on protein stability between both proteins. Interestingly, the protein bands in strains with no clp1 expression were blurred and had higher migrating bands compared to strains with clp1 expression (Fig 3.3A and 3.4A and B), suggesting that Cib1 undergoes a posttranslational modification in a clp1-dependent manner.

\subsubsection{Cib1 phosphorylation is reduced in a Clp1-dependent manner}

Regulation of transcription factors is not only limited to their transcriptional control of upstream activators or repressors. In a multitude of biological processes involved in developmental or cell cycle programs, post-translational modifications of transcription factors play an essential role to adjust the downstream transcriptional output. Alongside other studied post-translational modifications of transcription factors such as SUMOylation, ubiquitination, acetylation, glycosylation and methylation, the most rapid alteration of transcription factor modification to adapt to changes in the environment are provided by protein phosphorylation or dephosphorylation. Alterations in the phosphorylation pattern of transcription factors can affect their retention time in the nucleus, protein half-life, interaction with co-factors or DNA binding specificity (Whitmarsh and Davis, 2000; Filtz et al., 2014). Such a change in DNA binding specificity was shown in U. maydis for Prf1 (pheromone response factor 1). Phosphorylation of Prf1 by the protein kinase A, Adr1, leads to expression of the $a$ mating type genes whereas phosphorylation of Adr1 and the MAPK Kpp2 on different phosphorylation sites of Prf1 leads to activation of the $b$ genes (Kaffarnik et al., 2003).

In the present study, a phosphatase assay with Cib1 showed that Cib1 phosphorylation is reduced upon clp1 expression (Fig 3.5). In correlation with the observation of increased Cib1 stability by Clp1 induction (Fig 3.4A and B), these data indicate that an unphosphorylated state of Cib1 may be crucial for its stability. In S. cerevisiae, the UPR regulator Hac1p and the transcriptional activator of amino acid 
biosynthesis Gnc4p, are phosphorylated upon transcriptional initiation by Srb10p, a subunit of the $\mathrm{SRB} /$ mediator module of the RNA polymerase II holoenzyme. Phosphorylation marks Hac1p and Gcn4p for recognition by SCF(Cdc4) ubiquitin ligase leading to subsequent proteasomal degradation of the bZip transcription factors, which is referred to as the "black widow" model (Pal et al., 2007; Irniger and Braus, 2003; Kornitzer et al., 1994; Tansey, 2001; Chi et al., 2001). The LC-MS analysis revealed, that four possible phosphorylation sites of Cib1, Ser22, Ser33, Thr381, and Ser468, are differentially phosphorylated upon clp1 induction (Chapter 3.1.5). In particular, Ser22 and Ser33 seemed to be higher or not phosphorylated, respectively, in a Clp1-dependent manner. The phosphorylation sites, Thr381 and Ser468 were less phosphorylated upon clp1 induction. Ser468 is predicted to be phosphorylated by a p38 mitogen-activated protein kinase (Appendix Table 1). In S. cerevisiae, the p38 MAPK Hog1p (high osmolarity glycerol $\underline{1}$ ) of the HOG can be activated by osmotic stress (Schüller et al., 1994) or heat stress (Winkler et al., 2002) and is crucial for freeze protection (Panadero et al., 2006). In Botrytis cinerea, strains with deletion of the HOG1 homolog BcSAK1 were unable to penetrate the unwounded plant surface (Segmüller et al., 2007). However, it is unclear if the homolog of Hog1 in U. maydis contributes to virulence. In mammals, the p38 MAPK is described as a stress-activated protein kinase (SAPK) involved in different physiological processes such as inflammation response, cell cycle regulation, apoptosis or development and are activated upon ER stress (Coulthard et al., 2009; Matsuzawa et al., 2002). In addition, it was demonstrated in liver cells of mice that p38 MAPKdependent phosphorylation of $\mathrm{Xbp} 1^{\mathrm{s}}$ promotes nuclear translocation of $\mathrm{Xbp} 1^{\mathrm{s}}$, after a fasting period and subsequent refeeding of the mice (Coulthard et al., 2009). Microscopic analysis of the Cib1-GFP fusion protein revealed in addition to the expected nuclear localization a cytoplasmic signal upon clp1 induction (Fig 3.2). Hence, it appears possible that Cib1 shuttles between nucleus and cytosol in Clp1 and/or phosphorylation-dependent manner. In mammals, nucleocytoplasmic shuttling was reported for FoxO1, where neurotrophic factors promote nuclear exclusion of the transcription factor by affecting its phosphorylation pattern (Gan et al., 2005). Clp1 could support the nuclear export of Cib1 by inhibition of phosphorylation at specific residues. The phosphosites Thr381 and Ser468 are located near or in the Clp1 interaction domain, respectively, suggesting a steric inhibition of phosphorylation by Clp1. Thus, this could indirectly affect gene expression by translocation of Cib1 to the cytoplasm. This question could be addressed in future studies by monitoring the subcellular localization of cib1 phosphorylation mutants upon clp1 induction. In the present study, however, an infection assay of cib1 phospho-null or phosphomimic mutants did not show a significant change of infection symptoms compared to the wildtype (Fig 3.7). Since cib1-GFP fusion constructs were used to study the impact of the phosphomutations, it is possible that stabilizing properties of the GFP fusion (Janczak et al., 2015) diminished the virulence-dependent effects of the introduced phosphomutations. Hence, infection assays of cib1 phosphomutants should be repeated with a smaller fusion protein or no tag at all to counteract the positive effects of protein tags on target protein stabilization. However, protein stability of these Cib1 phosphorylation-mutants was not examined, yet. Phosphorylation of transcription factors often 
occurs in posttranslational crosstalk prior to ubiquitination, leading to degradation of the respective protein by proteasomal-degradation (Magnani et al., 2000; Treier et al., 1994; Fuchs et al., 1996). It remains unclear if phosphorylation of Cib1 affects its function or promotes degradation, which should be addressed in future studies. In a previous study, a cib1 $1^{433}$ mutant strain, lacking the Clp1-interaction domain (Clp1-ID, Fig 3.6) did not show modulation of the UPR target genes upon clp1 induction (Heimel et al., 2013). It will be quite interesting if destabilization of Cib1 occurs by phosphorylationdependent degradation, which may be counteracted by masking of possible phosphosites of Cib1 by Clp1 interaction.

\subsection{Modulation of the UPR in U. maydis is controlled by Clp1 4.2.1 Clp1 is modulating the transcriptional output of the UPR}

For many pathogenic fungi, such as Aspergillus fumigatus (Richie et al., 2009), Cryptococcus neoformans (Cheon et al., 2014), Candida albicans (Wimalasena et al., 2008), Alternaria brassicicola (Joubert et al., 2011) or Ustilago maydis (Heimel et al., 2013; Hampel et al., 2016) the unfolded protein response is essential for virulence. The ER represents a hub where most of the secreted and transmembrane proteins are post-translationally modified and folded, which is further supported by the UPR, governing the folding capacity of the ER and restoring ER homeostasis (Karagöz et al., 2019). An adjustable UPR is essential since hyperactivation of the UPR causes apoptosis (Woehlbier and Hetz, 2011). Modulation of the UPR can be achieved via different ways such as iron depletion and repletion (Cohen et al., 2017), lipid bilayer stress (Halbleib et al., 2017), phosphorylation of Ire1 (Welihinda et al., 1998), and regulation of Hac1 protein or mRNA stability (Tsvetanova et al., 2012; Pal et al., 2007; Glazier et al., 2015). Especially during plant infection of U. maydis, an adjusted UPR supports the biotrophic phase, where an unregulated or hyperactive UPR is deleterious and might trigger cell death. The developmental regulator Clp1 mediates UPR adaptation through direct interaction with Cib1, resulting in strongly elevated ER stress resistance (Heimel et al., 2013).

By RNAseq based transcriptome analysis of the UPR in U. maydis, 65 upregulated UPR core genes were identified (Fig 3.8). Most of these genes can be grouped into the ER-associated processes such as "unfolded protein response”, "stress response”, "protein folding and stabilization” and "protein/peptide degradation” (Fig 3.9), which are important for adaptation of the secretory pathway during increased ER stress (Travers et al., 2000; Arvas et al., 2006; Carvalho et al., 2012). Genes of the identified UPR core set are shown to be important for ER resistance in $U$. maydis and other fungi such as the ER chaperones, bip1 (UMAG_15034), also an interactor of Ire1 (Okamura et al., 2000; Jung et al., 2013) and lhs1 (UMAG_00904) (Yi et al., 2009), as well as dnj1 (UMAG_05173), an ER co-chaperone (Hampel et al., 2016), ero1 (UMAG_05219), an ER oxidoreductase (Frand and Kaiser, 1998), pd1 (UMAG_10156), a protein disulfide isomerase (Marschall and Tudzynski, 2017) as well as the UPR 
marker gene UMAG_11594 (K. Heimel pers. communication). The UPR core genes UMAG_05898/der1 and UMAG_00542/hrd1, which are core members of the ERAD pathway and are implicated in degradation of misfolded proteins in the ER lumen and the ER membrane (Ruggiano et al., 2014), were shown to be upregulated upon ER stress before in budding yeast (Travers et al., 2000). Three members of the signal peptidase complex (SPC), UMAG_00481, UMAG_15029, homologs of SEC11, SPC3 in yeast, respectively, as well as UMAG_06089, a homolog of the mammalian SPC25, were identified as UPR core genes. It was shown that members of the signal peptidase complex are essential for many organisms (La Rosa et al., 2004; Fang et al., 1997; Meyer and Hartmann, 1997). Moreover, the yeast homolog of SPC3 in Colletotrichum graminicola is implicated in virulence (Thon et al., 2002). Proper effector secretion is essential for pathogenic fungi to compete with the defense response of their hosts (Lo Presti et al., 2015). Most of the UPR core genes are functional categorized to ER and UPR-related functions (Fig 3.9) and are upregulated in planta (Fig 3.10) (Lanver et al., 2018), which supports the model of a UPR that might be involved in the effector production, translocation into the ER or maturing of precursor effector proteins in the ER.

The RNAseq experiment identified UPR target genes and revealed the clp1-dependent modulation of these genes (Fig 3.8) on a genome-wide level. 35 of the 65 identified UPR core genes, were not repressed by clp 1 induction whereas 30 of these genes were downregulated upon $c l p 1$ expression. In line with previous studies (Heimel et al., 2013), the UPR core genes bip1, lhs1 and mpd1 showed reduced expression levels during a clp1-dependent modulation of the UPR. It was hypothesized that genes that are not repressed upon Clp1 induction might be important for virulence of $U$. maydis. Most of these 35 UPR core genes have not been studied before and were subjected to a gene deletion screen, to examine ER stress resistance and monitor pathogenicity in a plant infection assay (Chapter 4.3.1).

\subsubsection{Clp1 does not alter DNA binding of Cib1}

In Hampel et al. (2016), a putative DNA binding motif of Cib1 was predicted based on an in silico promoter prediction and qChIP analysis (Hampel et al., 2016). To identify direct Cib1 regulated genes, a comparative ChIPseq analysis, including a strain expressing clp1, was performed and correlated with the UPR core gene set obtained by RNAseq analysis ( $\log 2 \mathrm{FC} \geq 1)$. This generated a list of 91 UPR core gene promoters present in the WT and the $\mathrm{P}_{\text {crg }}:$ clp1 condition. The identified Cib1 DNA binding site/UPRE by MEME-ChIP (Fig 3.12, WT, TGACGTGGAAG) is highly similar to the CREB3-like (TGCCACGTGGCA) and the Hac1-homolog XBP1 (TGACGTGGA) consensus sequence in higher eukaryotes (Jolma et al., 2013; Yamamoto et al., 2004). Moreover, the DNA binding motif of Hac1-like proteins might be similar, since only the bZip domain of the highly divergent Hac1-like proteins is conserved (Weirauch et al., 2014). In Drosophila melanogaster, the bZip transcription factor and Hac1homolog, CrebA, a major regulator of the secretory pathway, is upregulating genes of the general secretory machinery and tissue-specific secreted cargo (Fox et al., 2010). In U. maydis interaction of 
Cib1 with Clp1 leads to an increased ER stress tolerance, which further supports efficient effector secretion during biotrophic growth in planta. Moreover, the effector genes pit2 and tin1-1 and the cochaperone dnj1 showed induced cib1-dependent expression under ER stress (Heimel et al., 2013; Hampel et al., 2016; Lo Presti et al., 2016). It can be speculated that a development specific UPR in $U$. maydis increases the production and secretion of effectors by directly regulate specific genes. These genes might be present in the list of the 91 UPR core gene promoters, which should be investigated in future research.

The consensus sequence of Cib1 during expression of Clp1 was almost identical, but shorter, (Fig 3.12,

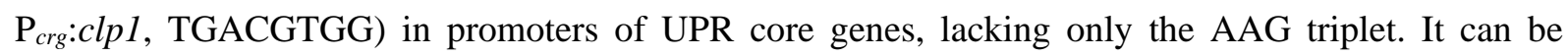
speculated that the Clp1 interaction could interfere with the DNA binding affinity of Cib1 by a physical or post-translational alteration of Cib1, such as the reduced phosphorylation during clp1 expression observed in this study (Chapter 3.1.4). It was shown in higher eukaryotes that the DNA binding affinity of $\mathrm{Cys}_{2} \mathrm{His}_{2}$ zinc finger proteins decreased more than 40-fold upon phosphorylation (Jantz and Berg, 2004). In the present study, UPR core genes with the highest gene regulation showed increased promoter scores during clp1 expressing conditions (Fig 3.14). Therefore, expression of these genes in cib1 phosphorylation mutant strains should be examined in future research.

The UPR target genes bip1, cib1, lhs1 and dnj1 investigated in previous studies (Heimel et al., 2013; Lo Presti et al., 2016) were in the top 20 list (Table 3.2) with the highest promoter scores. This indicates a correlation between high promoter scores and regulation of UPR target genes with high expression levels during ER stress. A predicted UPRE (TGCCACGT[C/G][G/T]) in the promoters of the effector genes tin1-1 and pit1/2 was validated by qChIP analysis in a previous study (Hampel et al., 2016) and is highly similar to the UPRE motif identified by the MEME-ChIP analysis in the present study (Fig 3.12, WT, TGACGTGGAAG). However, differential gene expression was only observed for pit1 and not for tin1-1 (Appendix Fig 7.1 and Appendix Table 2). In contrast, DNA binding of Cib1 was only identified in the tin1-1 promoter (Appendix Fig 7.1). It is possible that this is based on the different strain backgrounds used in Hampel et al. (2016)(SG200) and this study (JB1). In contrast to the SG200 strain, expressing an active b-heterodimer, the $b$-locus in the JB1 strain is deleted (Scherer et al., 2006). The JB1 strain was used in this study for RNAseq and ChIPseq experiments to avoid the combinatorial effects of different transcriptions factors. Expression of pit1/2 is controlled by the $b$-dependent transcription factor Hdp2 (Lanver et al., 2014) suggesting, that a genetic interaction between Cib1 and Hdp2 might foster the expression of pit1/2. 


\subsection{Spp1 is a novel pathogenicity factor}

\subsubsection{Deletion of UPR genes modulated by Clp1 induction revealed Spp1 as a novel virulence factor in $U$. maydis}

Clp1-dependent modulation of the unfolded protein response counteracts a hyperactive and deleterious UPR (Heimel et al., 2013). In this study, a comparative RNAseq analysis revealed 65 UPR core genes upregulated in a cib1-dependent manner. Moreover, 35 of these genes showed an increased or an unchanged expression upon clp1 induction (Chapter 3.2.2). It was hypothesized that genes of this UPR core gene subset might be necessary for ER stress resistance or during pathogenic development. To test this assumption, deletion mutants of these UPR core genes were generated and subjected to ER stress(Fig 3.16) as well as plant infection-assays (Fig 3.15). Three of those genes turned out to be essential for growth (Chapter 3.3.1), all of which are components of the signal peptidase complex that mediates signal peptide cleavage and translocation of proteins into the ER lumen (Paetzel et al., 2002). In yeast, disruption of some of the described subunits of the signal peptidase complex results in non-viable mutants (Meyer and Hartmann, 1997). However, upregulation of this complex during ER stress is in line with the increased secretory demand during effector secretion of U. maydis during plant colonization (Müller et al., 2008; Lanver et al., 2018).

Surprisingly, the deletion mutants of the remaining 29 UPR core genes were not impaired in ER stress resistance (Fig 3.16), suggesting that they are not crucial for ER stress resistance. In the plant infection screen, 26 of the deletion mutants were not and 2 only slightly impaired in pathogenicity (Fig 3.15). Hence, some of the encoded proteins might have redundant or overlapping functions during ER stress or during in planta growth that might support the robustness of plant infection of $U$. maydis (Lachowiec et al., 2016). This assumption is mirrored by the functional redundancy of effector genes observed in U. maydis and other pathogenic fungi (Lanver et al., 2017).

Alternatively, and as previously hypothesized (Heimel et al., 2013), it might also be possible that the main function of clp1-dependent UPR modulation is primarily to prevent the deleterious overexpression of the identified UPR core genes. In recent studies, overexpression of the spliced form of cib1 led to hyperactivation of the UPR and increased ER stress sensitivity (Heimel et al., 2013).

Deletion of the gene, encoding signal peptide peptidase spp1 did not affect ER stress resistance (Fig 3.16) or impaired cell wall integrity (Appendix Fig 7.4) but resulted in a complete loss of virulence (Fig 3.15), suggesting that spp1 is a crucial factor of fungal virulence. Bioinformatic analysis predicted that Spp1 is a conserved signal peptide peptidase, crucial for intramembrane cleavage of left-over signal peptides in the ER membrane (Weihofen et al., 2002). Spp1 has a conserved domain structure containing 9 TMDs as well as the catalytically active site comprised of a YD and a GLGD motif and the potential substrate binding motif QPALLY. In contrast to the highly similar Presenilins (Weihofen et al., 2002), a subgroup of aspartic membrane proteases, SPPs have an inverted topology of the active and substrate 
binding site (Sato et al., 2008). This correlates with the substrate-specificity of signal peptides, by only accepting type II transmembrane domains (Weihofen et al., 2002). Fluorescence microscopy revealed that Spp1 is located at perinuclear ER and cortical ER structures (Fig 3.20). spp1 is highly expressed after UPR activation (Appendix Table 2, WT vs. WT +TM, log2FC 2.93), which was not described before in other organisms. This result is in agreement with the upregulation of spp1 during in planta colonialization of U. maydis (Lanver et al., 2018). However, expression spp1 was unaffected by the

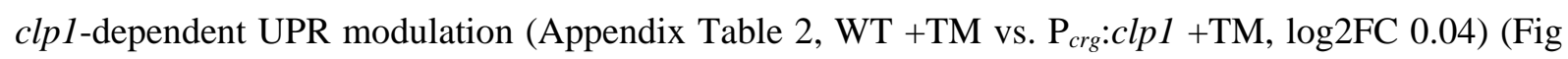
3.10), suggesting that stable expression of spp1 might be important for in planta growth.

Deletion of spp1 could be complemented by expression of SPPs from other smut fungi such as Srspp1 of S. reilianum, Uhspp1 of U. hordei or the human HM13/SPP (Fig 3.25). In contrast to SPPs from closely related species, which regained full virulence after introduction in the $\Delta$ spp1 strain, the human HM13 only partially restored virulence, although in a dose-dependent manner (Fig 3.25). The human HM13 cDNA clone was not codon optimized for $U$. maydis, which is probably the reason for the dosedependent effect on virulence. Codon optimization of heterologous genes is a common procedure to improve expression levels and increase the yield of the respective protein in biotechnology (Hu et al., 2013; Tokuoka et al., 2008; Wang et al., 2015). Taken together, the bioinformatic analysis, the subcellular localization and complementation of spp1 deletions strains with the well-studied human SPP ortholog, suggests that Spp1 is a bona fide SPP. Moreover, Spp1 is the only predicted SPP in the genome of $U$. maydis, whereas higher eukaryotes harbor up to five members of the SPP family, cleaving different kinds of substrates in several tissues and cell compartments (Fluhrer et al., 2009; Voss et al., 2013). This argues against the possibility that other SPPs in the genome of $U$. maydis cover the loss of spp1 during vegetative growth or ER stress adaptation (Fig 3.16 and 3.21). Complementation of the spp1 deletion with the Spp $1^{\mathrm{D} 279 \mathrm{~A}}$ mutant did not restore virulence of the strain in plant infection assays, implicating that the catalytical activity is crucial for pathogenicity. Mutation of the conserved aspartates in the YD/GLGD motif was shown to abolish cleavage of known SPP substrates (Boname et al., 2014).

The deletion mutant of spp1 is apathogenic in plant infection assays (Fig 3.15 and 3.25). However, the spp1 deletion mutant was still able to invade the plant surface (Fig 3.22). This phenotype is similar to those observed by $\Delta c i b 1$ and $\Delta c l p 1$ strains (Heimel et al., 2013), suggesting that the time point of the Spp1 function is simultaneously or shortly after UPR activation in planta. The stunted fungal growth in planta might be the result of the inability to suppress plant the plant defense response or the failed expression of hydrolytic enzymes to degrade the plant cell wall. In addition, it was observed that the spp1 deletion strain is forming hyphal constrictions at areas traversing plant cells (Fig 3.22, arrows). The biomass of $\Delta$ spp1 strains in planta is significantly decreased (Fig 3.23). Biotrophic pathogens like U. maydis typically attempt to suppress the salicylic (SA) and induce the jasmonic acid (JA)-mediated plant responses (Glazebrook, 2005). Expression of several pathogen-related plant defense genes of the SA pathway was highly induced upon infection with the $\Delta s p p 1$ or the $s p p 1^{\text {D279A }}$ strain, whereas 
expression of JA-marker genes was suppressed (Fig 3.29 and Appendix Fig 7.3). In agreement with this result, the formation of reactive oxygen species (ROS) in these mutants was strongly increased (Fig 3.26), since SA-related genes trigger ROS production upon pathogen attack (Kawano, 2003). In previous studies, the NADPH-oxidase inhibitor diphenyleneiodonium chloride (DPI), was used to counteract the $\mathrm{H}_{2} \mathrm{O}_{2}$ sensitivity of $U$. maydis mutants (Molina and Kahmann, 2007). However, the addition of DPI to inoculated spp1 deletions strains was not able to restore pathogenicity and did not affect on in planta growth (Fig 3.28A and B). Moreover, spp1 deletions trains were not sensitive to $\mathrm{H}_{2} \mathrm{O}_{2}$ stress during vegetative growth (Fig 3.27), indicating that Spp1 is not involved in the downstream response to $\mathrm{H}_{2} \mathrm{O}_{2}$ stress.

\subsubsection{Spp1 supports the establishment of the biotrophic interaction in planta}

In U. maydis, Spp1 is specifically required during growth in planta and deletion of spp1 did not impair vegetative growth or ER stress resistance. It is known from other organisms, that the general function of SPP is to remove left-over signal peptides in the ER membrane after processing of the translocated precursor protein by the signal peptidase complex (SPC). However, besides the clearing of signal sequence remnants of the ER membrane, SPPs are able to process different types of substrates containing a type II transmembrane domain (Chen et al., 2014; Avci et al., 2014; McLauchlan et al., 2002; Lemberg and Martoglio, 2004; Baldwin et al., 2014). It is tempting to speculate that Spp1 produces bioactive products with a virulence-specific function by cleavage of membrane-bound proteins during $U$. maydis plant infection. In Plasmodium falciparum, the use of pharmacological inhibitors of PfSPP's proteolytic activity inhibits intraerythrocytic development by blocking the ERAD-dependent degradation of unstable proteins (Harbut et al., 2012). In human, SPP is also implicated in ERAD by interacting with Derlin1 and the E3 ubiquitin ligase TRC8. Interaction of SPP with members of ERAD leads to cleavage of the membrane-bound $\mathrm{XBP} 1^{\mathrm{u}}$. Furthermore, subsequent liberation of $\mathrm{XBP} 1^{\mathrm{u}}$ leads to proteasomal degradation of XBP1 ${ }^{\mathrm{s}}$ and thereby fine-tuning the UPR signaling (Chen et al., 2014). In S. cerevisiae, the SPP Ypf1p interacts with the E3 ubiquitin ligase Doa10p and the ERAD factor Dfm1p to degrade Zrt1p, a high-affinity zinc transporter, in a zinc-dependent manner (Avci et al., 2014). In U. maydis, genes encoding major components of the ERAD complex were deleted in different combination to assess their contribution to virulence during plant infection and ER stress resistance. However, deletion of ERAD components did not impair pathogenicity and ER stress resistance (Fig 3.30 and 3.31). In A. fumigatus, multiple deletions of genes encoding ERAD component did not affect pathogenicity, but showed hypersensitivity to ER, thermal, and cell wall stress (Krishnan et al., 2013). In contrast to this study, the fungal UPR of $U$. maydis during in planta growth is not elevated in spp1 deletion strains (Fig 3.36), indicating no increased UPR due to a higher load of unfolded proteins in the ER. Taken together, both results suggest that the virulence-specific Spp1 function in $U$. maydis is not connected to the ERAD pathway. In A. nidulans and A. fumigatus, the sterol regulatory element-binding protein (SREBP) srbA 
is sequentially cleaved by a Dsc complex-associated proteolysis following SppA cleavage. Cleavage by SppA releases the bHLH transcription factor SrbA that activates genes essential for hypoxia adaptation (Bat-Ochir et al., 2016; Willger et al., 2008). In this study, the srbA homolog of U. maydis, srb1 (UMAG_05721) was deleted. However, deletion of srb1 had no impact on virulence and ER-stress (Fig 3.32). Moreover, the $\Delta$ spp1 strain was not impaired in growth under hypoxic conditions (Fig 3.33), indicating that Spp1 is dispensable for hypoxia adaptation.

In humans, during the maturation of the hepatitis $C$ virus, the ER membrane-embedded hepatitis core protein C is processed by the HM13 (McLauchlan et al., 2002; Filipe and McLauchlan, 2015). However, this can be excluded for the $U$. maydis Spp1 function, because a similar mechanism does not exist in fungi or plants. The human SPP was also found to be involved in MHC class I antigen presentation on the cell surface. Major histocompatibility complex (MHC) class I molecules are produced of signal peptides by subsequent cleavage of SPP liberating the fragment to the cytosol. Thus, the transporter associated with antigen processing (TAP) protein complex transports the fragment back into the lumen of the ER where it binds to an HLA-E molecule. Transport of this complex via the secretory pathway to the cell surface leads to protection against natural killer cells (Weihofen and Martoglio, 2003; Oliveira et al., 2013). Participation of MHC molecules in fungi can be excluded since MHC molecules are only present in jawed vertebrates (Flajnik and Kasahara, 2001).

Plant defense response suppression by biotrophic pathogens is required to establish a compatible interaction with their hosts (Lo Presti et al., 2015) and effectors that suppress the PTI are well studied in plant pathogens (Irieda et al., 2019; Boller and He, 2009; Park et al., 2012; Liu et al., 2016) The data generated in this study strongly suggest that Spp1 function is necessary to overcome the host innate immune response. The loss of spp1 during plant infection resulted in an increased expression of plant defense genes (Fig 3.29 and Appendix Fig 7.3) as well as an oxidative burst by the plant (Fig 3.26). However, spp1 deletion did not impair general the effector secretion, since the effectors Pit2 (Doehlemann et al., 2011), Pep1 (Hemetsberger et al., 2012), Tin2 (Tanaka et al., 2014) and Cmu1 (Djamei et al., 2011) monitored in the secretion assay, were still secreted under axenic conditions (Fig 3.34 and 3.35). Nevertheless, PAMP-triggered immunity (PTI) of the plant might be suppressed by secreted, Spp1-derived molecules to avoid the generation of ROS and hypersensitive response (HR). It is possible that this is mediated by small, secreted peptides previously processed and activated by Spp1. The effectors Pit2, Pep1 and Cmu1 were possible substrates/targets for an Spp1 mediated activation, in relation to the observed upregulation of SA-related genes and the ROS generation in plants infected with spp1 deletion strains. Pit2 inhibits maize cysteine proteases that promote SA-related plant defense, Pep1 suppresses the plant immunity by inhibiting POX12, a peroxidase in maize generating ROS upon pathogen detection and the chorismate mutase Cmu1 is secreted in high amounts to counteract the SAinduced immune responses (Lo Presti et al., 2015). Interestingly, secretion of Tin2 and Pep1 and processing of Pep1 and Cmu1 is dependent on ER stress and in an Spp1-independent way (Fig 3.35). 
This indicates coordination and modulation of the general effector secretion by the UPR, which should be addressed in future studies.

\subsubsection{Spp1 is a direct Cib1 target with functional UPREs in its promoter}

spp1 is specifically induced during in planta growth (Lanver et al., 2018), which is probably connected to its upregulation during UPR activation observed in axenic conditions (Fig 3.10 and Heimel et al. (2013)). Furthermore, spp1 is induced in a cib1-dependent manner, indicated by fluorescent microscopy

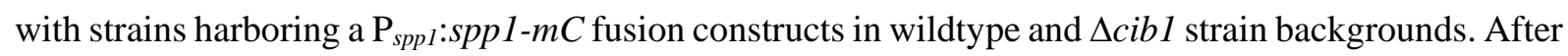
UPR activation, an increased fluorescence signal was only observed in strains with wildtype background (Fig 3.20), indicating a direct cib1 regulation. In a recent study, an in silico prediction of UPRE motifs in promoters of $U$. maydis effector genes as well as a subsequent qChIP analysis revealed the DNA binding motif of Cib1 in promoters of the effector genes pit1/2 and tin1-1. Strains harboring a deletion

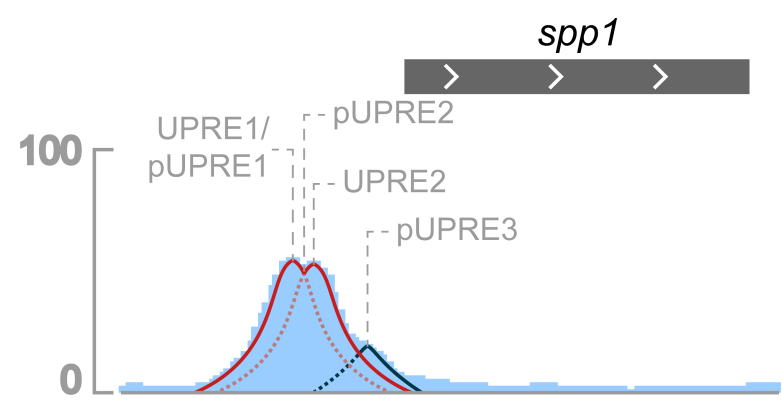

Figure 4.1: Schematic representation of the Cib1 binding site in the spp1 promoter. The depicted UPREs sequence (UPRE1 and UPRE2) were selected according to their local peak enrichment. Bioinformatic prediction revealed more UPREs (pUPRE2 and pUPRE3) in the spp1 promoter. Peak enrichment is indicated as solid red and blue bell curves. Overlapping area of the bell curves is indicated as dotted pink and blue lines. of the UPRE motif (TGCCACGTCG) in the pit1/2 promoter lost UPR-dependent pit1/2 gene expression and were significantly impaired in virulence (Hampel et al., 2016). In the present study, a genome-wide ChIPseq analysis of Cib1 binding sites was performed, to reveal directly Cib1 regulated UPR core genes as well as modulation of the Cib1 binding site by Clp1 expression (Chapter 3.2.3). The ChIPseq analysis revealed a strong Cib1 binding in the promoter of spp1 (Fig 3.13 and 4.1). However, promoter scores between wildtype (ps 260.82) and the clp1 expressing conditions (ps 261.41) were almost identical (Fig 3.13 and 3.14 and Appendix Table 3). This correlates well with the result of the RNAseq analysis, where expression of spp1 during ER stress conditions is not affected by clp1 induction (Appendix Table 2, WT +TM vs. $\mathrm{P}_{c r g}: c \operatorname{lp} 1+\mathrm{TM}$, log2FC = 0.04). Two potential UPRE motifs, UPRE1 (CTTCCACGTCT) and UPRE2 (GCGCTGTGCG) were identified in the spp1 promoter according to their local peak enrichment (Fig 4.1, red bell curves). Since deletion of UPREs in promoters of pit1/2 and tin1-1 affected gene expression under ER stress (for pit1/2 and tin1-1) and virulence of UPRE deletion strains (for pit1/2) in infected maize plants, strains harboring deletions of the potential UPRE motifs identified in the spp1 promoter were generated (Chapter 3.3.10). TM-induced expression of spp1 was significantly reduced in strains lacking one ( $\triangle \mathrm{UPRE} 1$ or $\triangle \mathrm{UPRE} 2)$ or both ( $\triangle \mathrm{UPRE} 1 / 2)$ of the identified UPREs (Fig 3.38), indicating that spp1 is a direct target of Cib1. Since expression of spp1 under ER stress condition was significantly reduced to basal expression levels, it can be speculated that the spp1 promoter comprises only two functional UPREs. The reverse 
complement sequence of the first UPRE1 is highly similar to the UPRE motif identified by MEMEChIP analysis (Fig 3.12, WT, TGACGTGGAAG). However, the second UPRE2 is only poorly conserved compared to the UPRE identified by MEME-ChIP (Fig 3.12, WT, TGACGTGGAAG) and thus might resemble a DNA binding site of a different, putatively interacting transcription factor of Cib1. Surprisingly, the UPRE deletion strains did not resemble the deletion phenotype of spp1 and were still pathogenic, but showed fewer infection symptoms than the wildtype (Fig 3.39). Integration of the UPRE deletion constructs was performed in the strain background of $\Delta$ spp 1 and was integrated via homologous recombination into the ip locus. It appears possible, that the chromatin structure of the ip locus (Brachmann, 2001) has a more relaxed and transcriptionally active conformation compared to the native genomic locus of the spp1 promoter. This can lead to increased basal expression levels of spp1, which may be sufficient for progression of in planta growth. To test this assumption, deletions of the identified UPREs should be performed in the spp1 promoter at the native genomic locus. Moreover, spp1 is fused to the fluorophore mCherry in constructs harboring the UPRE deletion in the spp1 promoter. Fusion with fluorescent tags could increase the protein stability of the target protein (Mestrom et al., 2019; Janczak et al., 2015). Thus, basal expression levels could be sufficient to generate potentially stabilized Spp1-mCherry proteins that fulfill the function of Spp1 during growth in planta. Furthermore, it cannot be excluded that other transcription factors can bind the spp1 promoter during plant infection and activate gene expression of spp1. Since the full-length spp1 promoter $(1.4 \mathrm{~kb})$ was used for the UPRE deletion assay, truncated variants of the promoter should be generated. The UPRE1 is located 433 bp upstream of the tss, which may mark the minimal promoter size (433 bp) to induce spp1 expression under ER stress conditions.

In silico prediction of UPREs in the SPP promoters of closely related smut species predicted three UPREs (pUPRE1: CTTCCACGTCT, pUPRE2: TGACGTGTTGG, pUPRE3: TTGACACGTCG) for U. maydis spp1 promoter, and one in the SPP promoters of S. reilianum (pUPRE1: CTTCCACGTCT), U. hordei (pUPRE1: CTGCCACGTCT) and U. bromivora (pUPRE1: CTGCCACGTCT). pUPRE1 (Pvalue $6.3 \times 10^{-6}$ ) of $U$. maydis is identical with the UPRE1 identified in the UPRE deletion assay

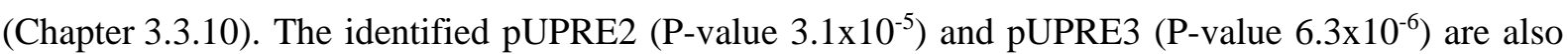
highly similar to the motif used for the prediction analysis. It will be interesting to examine if pUPRE2 and pUPRE3 are crucial for expression and/or virulence of spp1. Interestingly, the pUPRE1 identified in the Srspp1 promoter of $S$. reilianum is identical with pUPRE1 of U. maydis and the position of the pUPRE1 (424 bp upstream of tss) of S. reilianum are as well conserved in U. hordei and U. bromivora (433, 384 and 373 bp upstream of tss, respectively). RNAseq analyses of $U$. hordei and $U$. bromivora revealed that expression of the spp1 homologs Srspp1 and Ubspp1 was increased during biotrophic growth (Ökmen et al., 2018; Rabe et al., 2016), which suggests that the crosstalk between the UPR and the Spp1-function during biotrophic growth a might not be restricted to U. maydis. 


\subsubsection{UMAG_02578 is a potential Spp1 interaction partner identified by LC- MS analysis}

SPPs are described to be involved in different cellular processes such as the removal of remnant signal sequences (Weihofen et al., 2002), ERAD dependent degradation of misfolded proteins (Harbut et al., 2012; Chen et al., 2014), hypoxia adaptation (Bat-Ochir et al., 2016; Willger et al., 2008) or production of MHC class I molecules (Weihofen and Martoglio, 2003; Oliveira et al., 2013). Spp1 is not involved in ERAD (Chapter 3.3.7), $\mathrm{H}_{2} \mathrm{O}_{2}$ detoxification (Chapter 1.3.6) and growth under hypoxia (Chapter 3.3.7). To identify potential interaction partners of Spp1 strains expressing Spp1-GFP and the Spp1 $1^{\text {D279A }}$-GFP fusion proteins were used for Co-IP experiments and subsequent LC-MS analysis.

This identified 11 proteins that were co-immunoprecipitated with Spp1-GFP and/or Spp1 $1^{\text {D279A }}$ (Fig 3.43). The identified proteins can be grouped into three groups: Proteins, which are upregulated (UMAG_06089, UMAG_02833, UMAG_04994, UMAG_06480, UMAG_10649), not regulated (UMAG_02016, UMAG_06273, UMAG_05433, UMAG_02578) or downregulated (UMAG_11590, 02266) in planta (Lanver et al., 2018). It can be speculated, that genes of the upregulated group are crucial for the function of Spp1 during in planta growth.

The protein UMAG_06089 is predicted to be a microsomal signal peptidase subunit and was identified as a member of the UPR core genes (Chapter 3.2.2). However, deletion of the gene is lethal for U. maydis. UMAG_06089 was only identified during ER stress conditions, which is in line with RNAseq data (Appendix Table 2, WT vs. WT +TM, log2FC 2.14). In mammals, the hetero-oligomeric signal peptide complex (SPC) consists of five different subunits, which promoting cleavage of the signal peptides of translocated proteins in the ER. It was reported that the SPC25 subunit is required for the interaction of SPC with the translocation machinery (Kalies et al., 1998). In addition, a homolog of the member of the Sec63 translocation complex, Sec66p in budding yeast (Feldheim et al., 1993), was identified as a potential interaction partner of Spp1 (Fig 3.43, UMAG_02833). Direct interaction of an SPP with SPC25 and Sec66 was not shown before. Since translocation of precursor proteins, cleavage of their signal peptide and processing of signal sequences by SPP is an intergradient process in the ER membrane, it seems most likely that this interaction is real. In human, SPP is interacting with TRAM1, a translocon-associated membrane protein (Oresic et al., 2009). Therefore, it can be speculated, that Spp1 is interacting with the translocation complex Sec63-Sec66. In addition, the signal peptidase complex is tethered to the translocation machinery via SPC25 which leads to an indirect interaction with Spp1.

The protein UMAG_02016 has a predicted Cytochrome b5-like Heme/Steroid binding domain and is related to Dap1p of S. cerevisiae. In S. pombe, Dap1 promotes normal sterol levels by interacting with Erg5 and Erg11, both required for ergosterol biosynthesis (Hughes et al., 2007). Erg5 and Erg11 are controlled by the sterol regulatory element-binding protein (SREBP) Sre1 (Todd et al., 2006). In 
A. nidulans and A. fumigatus, the SREBP SrbA is activated by its SPP SppA, which is required for hypoxia adaptation (Bat-Ochir et al., 2016). Moreover, the protein UMAG_06273 is related to the NADPH-cytochrome P450 reductase Ncp1p of S. cerevisiae, which is involved sterol biosynthesis and coordinately regulated with Erg11p (Turi and Loper, 1992). However, Spp1 is not required for hypoxia adaptation and the SrbA homolog in U. maydis Srb1 is not required for pathogenicity (Chapter 3.3.7). It can be speculated, that Spp1 indirectly regulates sterol levels in U. maydis via the Dap1p and Ncp1p homolog UMAG_02016 and UMAG_06273, respectively, by activation or degradation of both proteins. However, it remains unclear if sterol biosynthesis can be linked to the biotrophic growth of $U$. maydis. It will be interesting to elucidate if deletion of each gene is connected to the pathogenicity of $U$. maydis during plant infection.

The protein UMAG_05433 identified by the LC-MS analysis is related to Pmt4p of S. cerevisiae and is required for O-linked glycosylation of secretory and cell surface proteins (Sanders et al., 1999). Deletion of the PMT4 gene in C. neoformans resulted in an abnormal growth morphology, defective cell separation and attenuation of virulence (Olson et al., 2007). However, spp1 deletion mutants were not impaired in growth under cell wall stress conditions and showed a normal vegetative growth (Appendix Fig 7.4 and Fig 3.21). In U. maydis, deletion of UMAG_05433/pmt4 led to a loss of virulence during plant infection (Fernández-Alvarez et al., 2009) and the transmembrane protein Pit1 was identified as a Pmt4 target. Mannosylation of Pit1 by Pmt4 might be crucial for effector secretion, since deletion of pit1 results in avirulence of $U$. maydis strains during plant infection (Fernández-Álvarez et al., 2012). It can be speculated that the interaction between Spp1 and Pmt4 might important for mannosylation of effector proteins.

Surprisingly, the protein UMAG_02578 was only identified in conditions with the catalytically inactive Spp1 $1^{\text {D279A }}$-GFP mutant strain. To identify UMAG_02578 subcellular localization, it was fused to GFP and expressed with under the constitutive active otef promoter under normal and ER stress conditions. Under normal conditions, the UMAG_02578-GFP fusion protein can be observed at ER-like structures. However, upon ER stress an elevated nuclear fluorescence signal could be observed, indicating an ER stress-dependent accumulation at the nucleus (Fig 3.44). UMAG_02578 consists of a predicted transmembrane domain (Phobius) as well as a luminal heterokaryon incompatibility protein (Het-C) domain. However, the transmembrane domain seems only to be conserved homologs of UMAG_02578 in smut fungi. The het locus in filamentous fungi such as $N$. crassa regulating self/nonself-recognition during vegetative growth, crucial for the formation of a hyphal network. The fusion of cells occurs if het alleles are identical. However, if individuals have different het alleles, cells undergo programmed cell death, also termed as heterokaryon incompatibility (Glass and Dementhon, 2006; Glass and Kaneko, 2003). In N. crassa, 11 different heterokaryon incompatibility (het) loci exist (Perkins, 1988). One of the well-studied het loci is the multiallelic het-c locus, which consists of two genes, het-c and pin-c (partner for incompatibility with het- $\underline{c}$ ). The het-c gene encodes a 966 AA plasma membrane protein 
containing a HET domain and a signal peptide, whereas the pin-c encodes a protein with a HET domain. Moreover, nonself recognition and heterokaryon incompatibility are mediated by the interaction between Het-C and Pin-C (Sarkar et al., 2002; Saupe et al., 1996; Kaneko et al., 2006). However, genes encoding HET domains are absent in S: cerevisiae and S. pombe as well as in the basidiomycetes C. neoformans and Coprinus cinereus (Kaneko et al., 2006). However, the function of the HET domaincontaining protein UMAG_02578 in U. maydis is not yet described. In contrast to the plasma membranelocated Het-C of N. crassa, UMAG_02578 is located at ER-like structures (Fig 3.44), has a predicted transmembrane domain and has no predicted signal peptide. Moreover, UMAG_02578 is the sole gene encoding a HET domain in the U. maydis genome and might have a different function than the self/nonself recognition described in $N$. crassa. Different functions for HET domain-containing genes are described such as for $A$. nidulans, where TinC interacts with the mitotic kinase NimA. Expression of a TinC lacking an N-terminal region lead to defects in the cell cycle and colony growth. However, deletion or overexpression of full-length TINC did not reveal an identifiable phenotype (Davies et al., 2004). The latter could be similar to U. maydis UMAG_02578 since an infection of maize plants with UMAG_02578 deletion strains did not affect pathogenicity of $U$. maydis. It can be speculated that only the C-terminal, luminal domain of UMAG_02578 is bioactive, whereas the full-length protein may be constitutively processed by Spp1 to inactive the function of UMAG_02578.

Further research should address the function of these potential interaction partners of Spp1, during ER stress conditions and pathogenicity of U. maydis. It is quite intriguing that the pathogenicity of $U$. maydis is solely constituted by the conserved catalytic activity of Spp1. The Spp1 function is not related to ERAD, hypoxia adaptation or other identified SPP substrates. It can be speculated that either specific activation of Spp1 or cleavage of substrates that are expressed during in planta growth, cause plant defense suppression. Since an elevated fungal UPR could not be observed in plants infected with $\Delta$ spp1 strains, it is unlikely that an excess of left-over signal peptides in the ER membrane leads to growth inhibition in planta. It is possible that specific plant signal leads to the expression of specific substrates, that need to be cleaved by Spp1. Since it was possible to pull-down potential interaction partners of Spp1 in axenic culture, a co-immunoprecipitation of Spp1 from infected leaf tissue with subsequent identification of interaction partners by LC-MS should be performed, which may shed new light to the underlying mechanism. In summary, the results of this study revealed a novel pathway in fungal pathogens to suppress the host defense and establish a biotrophic interaction. 


\subsection{Model of the Clp1-dependent modulation of the UPR}

Fusion of two compatible sporidia leads to the formation of the b-heterodimer that initiates biotrophic growth of $U$. maydis on the plant surface of its host plant maize. Subsequently, both events (activation of the pheromone pathway during cell/cell fusion and the formation of the bE/bW complex) trigger and maintain a G2-cell cycle arrest. The active transcription factor bE/bW induces expression of genes encoding the developmental regulator Clp1 and the master regulator of the $b$-dependent transcriptional cascade Rbf1. Clp1 is stabilized after appressoria formation and plant penetration of the leaf surface by the central regulator of the UPR and bZip transcription factor Cib1. Thus, increased Clp1 levels repress the $b$-dependent gene expression by the interaction between Clp1 and the bE/bW heterodimer and Rbf1, resulting in a release of the G2 cell cycle arrest (Heimel et al., 2010a; Heimel et al., 2010b; Heimel et al., 2013). The results of the present study provide new insights into the Clp1-dependent modulation of Cib1 function during pathogenic development. In particular, a hypothetical model can be proposed in which Clp1 negatively affects the transcriptional activity of Cib1, reducing Cib1 phosphorylation and increasing the stability of Cib1 (Fig 4.2A). In this model, the interaction of Clp1 might either mask the Cib1 transactivation domain (Fig 4.2B), interfere with Cib1 homodimer formation (Fig 4.2C) or alter the subcellular localization of Cib1. (Fig 4.2D)

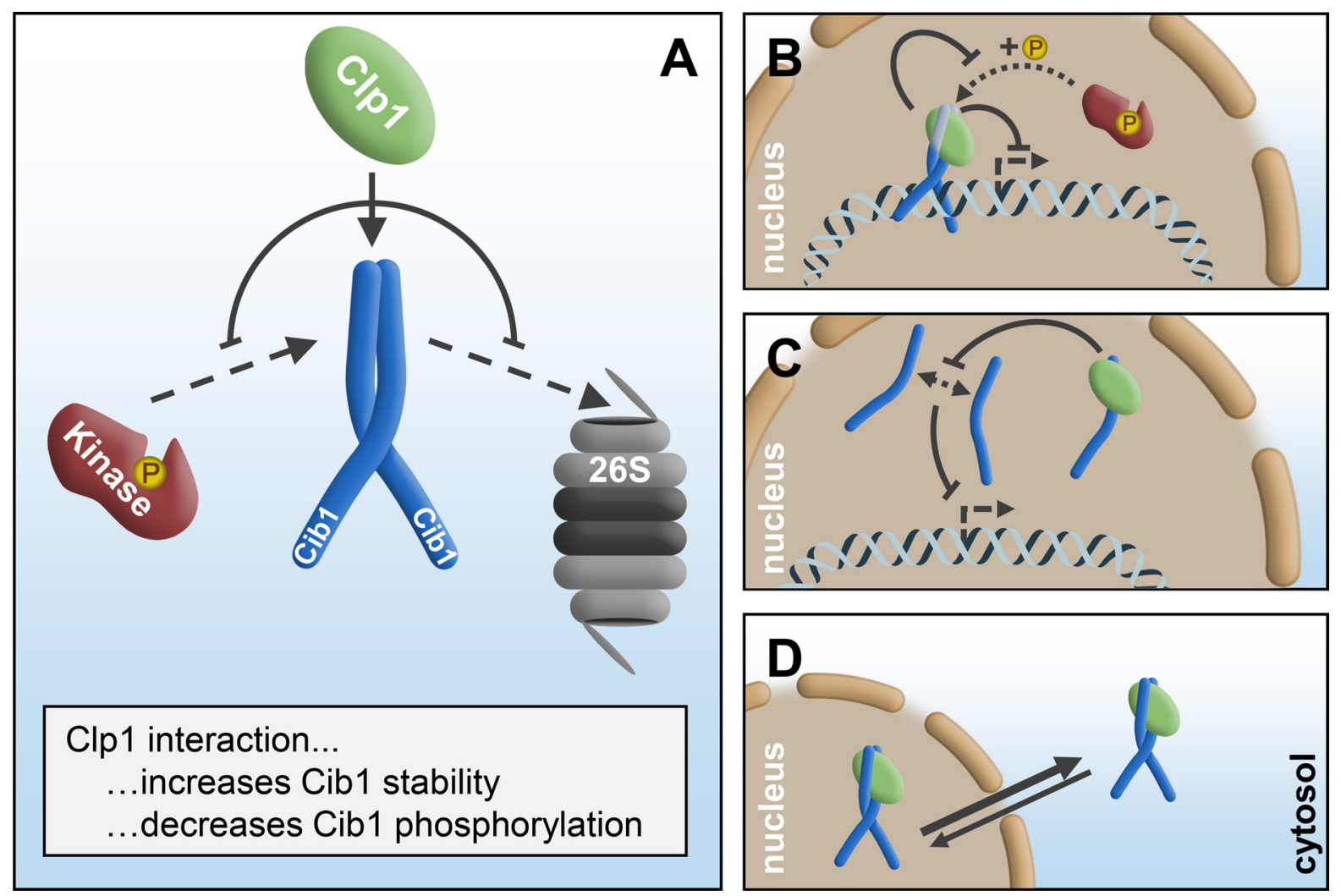

Figure 4.2: Model of the Clp1-dependent modulation of the Cib1 function. (A) The results of the present study revealed that the interaction of Clp1 with Cib1 increases Cib1 stability and decreases phosphorylation at the Clp1 interaction domain of Cib1. The reduced phosphorylation is probably a result of (B) Clp1-mediated masking of the Cib1 activation domain, preventing Cib1 phosphorylation by a yet unknown kinase. Thus, Clp1 interaction with Cib1 modulates the transcriptional activity observed in the expression levels of UPR core genes during clp1 induction. However, the Clp1-dependent modulation of gene expression can be achieved via different hypothetical mechanisms, such as (C) the Clp1-dependent inhibition of Cib1 homodimer formation or (D) the cytosolic export of Cib1 in a Clp1-dependent manner. 
The results of this study further support the idea of a mutual stabilization between Clp1 and Cib1 during the interaction. In Heimel et al. (2013) half-life of Clp1 was shown to be less than 30 min, which is much shorter than the Cib1 half-life (>180 min) upon clp1 induction (Fig 3.4A and B). Hence, the negative impact on the transcriptional activity of Cib1 by Clp1 interaction (Fig 4.2A) would be transient, but preventing a deleterious UPR gene expression. The expression of Clp1 and Cib1 is active throughout all stages of in planta growth (Heimel et al., 2010b; Heimel et al., 2013; Lanver et al., 2018), indicating that the UPR needs to be consistently adjusted. This mechanism could buffer the fluctuating demands on the ER caused by effector waves during biotrophic growth, to sustain a perpetual UPR gene expression. The underlying mechanism of Clp1-dependent modulation of the Cib1 phosphorylation pattern (Chapter 3.1.4 and 3.1.5) is still elusive and requires further research. It will be interesting to examine if Cib1 is phosphorylated during transcriptional regulation or by external signals in Clp1dependent manner. Further research should focus on the identified UPR core genes repressed by clp1 (Chapter 3.2.2). It can be speculated that the UPR in U. maydis can be divided into two states according to the Clp1-dependent modulation of UPR core genes. Thus, UPR core genes repressed by Clp1 sustain general UPR functions like protein folding or degradation of misfolded proteins. In contrast, UPR core genes that are positively affected by Clp1 may be composed of uncharacterized genes with potentially redundant virulence-specific functions as well as three core components of signal peptidase complex (Chapter 3.2.2), suggesting that the UPR might be adjusted to optimize effector secretion during in planta growth.

\subsection{Conclusion}

The present study shed new light on the Clp1-dependent modulation of the UPR, which was initially described in Heimel et al. (2013). It could be shown that crosstalk between the developmental regulator Clp1 and the UPR regulator Cib1, not only affects UPR gene expression but also changes physical properties of the bZip transcription factor Cib1 by increased protein stability and modulated phosphorylation. Transcriptomic analysis revealed a novel set of UPR genes regulated by Cib1 and modulated in a clp1-dependent manner. Genome-wide mapping of UPR elements in promoters was correlated with transcriptomic data and revealed directly Cib1 regulated UPR genes. However, no change in DNA binding specificity was observed in clp1 expressing conditions. Importantly, the novel pathogenicity factor Spp1 was identified as a direct Cib1 regulated gene, which is crucial for the pathogenicity of $U$. maydis. In summary, the data generated in this study indicates that Spp1 functions in a novel pathway to suppress the host defense response and establish in planta growth of biotrophic plant pathogens. 


\section{Materials and Methods}

\subsection{Material and sources of supply}

\subsubsection{Escherichia coli strain}

For vector cloning the Escherichia coli strain TOP10 (Invitrogen) was used, which is a derivative of the strain DH10B with the following genetic markers: F- mcrA $\Delta$ (mrr-hsdRMS-mcrBC) Ф80lacZ $\Delta M 15$ $\Delta l a c X 74$ recA1 endA1 araD139 $\Delta$ (ara leu) 7697 galU galK rpsL nupG $\lambda$ - (Casadaban and Cohen, 1980; Durfee et al., 2008).

\subsubsection{Ustilago maydis strains}

Table 5.1: Ustilago maydis strains used in this work

\begin{tabular}{|c|c|c|c|}
\hline Strain & Genotype & Resistance & Reference \\
\hline SG200 & a1:mfa2, bE1, bW2 & $\mathrm{Phleo}^{\mathrm{R}}$ & $\begin{array}{l}\text { Kämper et al. } \\
(2006)\end{array}$ \\
\hline JB1 & $a 1, \Delta b$ & $\mathrm{Hyg}^{\mathrm{R}}$ & $\begin{array}{l}\text { Scherer et al. } \\
\text { (2006) }\end{array}$ \\
\hline UV0151 & a1, $\Delta b, P_{\text {crg: }}: c / p 1$ & $\mathrm{Hyg}^{\mathrm{R}}, \mathrm{Cbx}^{\mathrm{R}}$ & $\begin{array}{l}\text { Scherer et al. } \\
(2006)\end{array}$ \\
\hline UKH6 & a1:mfa2, bE1, bW2, $\Delta c i b 1$ & $\mathrm{Phleo}^{\mathrm{R}}, \mathrm{Hyg}^{\mathrm{R}}$ & $\begin{array}{l}\text { Heimel et al. } \\
(2010 a)\end{array}$ \\
\hline UKH8 & $a 1, b 1, \Delta c i b 1$ & $\mathrm{Hyg}^{\mathrm{R}}$ & Heimel (2010) \\
\hline UMH72 & $a 1, \Delta b, \Delta c i b 1 \# 1$ & $\mathrm{Hyg}^{\mathrm{R}}, \mathrm{Nat}^{\mathrm{R}}$ & $\begin{array}{l}\text { Heimel et al. } \\
\text { (2013) }\end{array}$ \\
\hline UMH243 & $\begin{array}{l}\text { a1:mfa2, bE1, bW2, } \Delta \text { pit2, } \\
P_{\text {otef:pit2-mCherry (\#1) }}\end{array}$ & $\begin{array}{l}\mathrm{Phleo}^{\mathrm{R}} \\
\mathrm{Hyg}^{\mathrm{R}}, \mathrm{Cbx}^{\mathrm{R}}\end{array}$ & $\begin{array}{l}\text { Hampel et al. } \\
\text { (2016) }\end{array}$ \\
\hline UMH244 & $\begin{array}{l}\text { a1:mfa2, bE1, bW2, } \Delta \text { pit2, } \\
\text { Potef:pit2-mCherry (\#1), } \Delta c i b 1\end{array}$ & $\begin{array}{l}\text { Phleo }^{R} \text {, } \\
\text { Hygg }^{R}, \text { Cbx }^{R} \text {, } \\
\text { Nat }^{R}\end{array}$ & $\begin{array}{l}\text { Hampel et al. } \\
\text { (2016) }\end{array}$ \\
\hline UDM28 & ip $^{R}\left[P_{\text {otef: }}\right.$ GFP-3xHA]ip $p^{S}$ & $\mathrm{Phleo}^{\mathrm{R}}, \mathrm{Cbx}^{\mathrm{R}}$ & $\begin{array}{l}\text { pers. com. } \\
\text { D. Martorana }\end{array}$ \\
\hline UDM80 & a1:mfa2, bE1, bW2, cib1-GFP & $\mathrm{Phleo}^{\mathrm{R}}, \mathrm{Hyg}^{\mathrm{R}}$ & $\begin{array}{l}\text { pers. com. } \\
\text { D. Martorana }\end{array}$ \\
\hline
\end{tabular}

Table 5.2: Ustilago maydis strains generated in this work

\begin{tabular}{|c|c|c|c|}
\hline Strain & Genotype & Resistance & Source \\
\hline UNP6\#1 & a1:mfa2, bE1, bW2, $\Delta$ spp1 & $\mathrm{Phleo}^{\mathrm{R}}, \mathrm{Hyg}^{\mathrm{R}}$ & SG200 \\
\hline UNP6\#2 & a1:mfa2, bE1, bW2, $\Delta s p p 1$ & $\mathrm{Phleo}^{\mathrm{R}}, \mathrm{Hyg}^{\mathrm{R}}$ & SG200 \\
\hline UNP6\#3 & a1:mfa2, bE1, bW2, $\Delta$ spp1 & $\mathrm{Phleo}^{\mathrm{R}}, \mathrm{Hyg}^{\mathrm{R}}$ & SG200 \\
\hline UNP12\#1 & a1:mfa2, bE1, bW2, $\triangle U M A G \_12149$ & $\mathrm{Phleo}^{\mathrm{R}}, \mathrm{Hyg}^{\mathrm{R}}$ & SG200 \\
\hline UNP13\#1 & a1, $\Delta b, P_{\text {crg1:clp1, cib1-GFP }}$ & $\begin{array}{l}\mathrm{Hyg}^{\mathrm{R}}, \mathrm{Cbx}^{\mathrm{R}} \text {, } \\
\mathrm{Nat}^{\mathrm{R}}\end{array}$ & UVO151 \\
\hline UNP13\#2 & a1, $\Delta b, P_{\text {crg1:clp1, cib1-GFP }}$ & $\begin{array}{l}\mathrm{Hyg}^{\mathrm{R}}, \quad \mathrm{Cbx}^{\mathrm{R}}, \\
\mathrm{Nat}^{\mathrm{R}}\end{array}$ & UVO151 \\
\hline UNP13\#3 & $a 1, \Delta b, P_{\text {crg1:clp1, cib1-GFP }}$ & $\begin{array}{l}\mathrm{Hyg}^{\mathrm{R}}, \quad \mathrm{Cbx}^{\mathrm{R}} \text {, } \\
\mathrm{Nat}^{\mathrm{R}}\end{array}$ & UVO151 \\
\hline
\end{tabular}




\begin{tabular}{|c|c|c|c|}
\hline UNP14\#1 & $a 1, \Delta b$, cib1-GFP & $\mathrm{Hyg}^{\mathrm{R}}, \mathrm{Nat}^{\mathrm{R}}$ & $\mathrm{JB} 1$ \\
\hline UNP14\#2 & $a 1, \Delta b, c i b 1-G F P$ & $\mathrm{Hyg}^{\mathrm{R}}, \mathrm{Nat}^{\mathrm{R}}$ & $\mathrm{JB} 1$ \\
\hline UNP14\#3 & $a 1, \Delta b, c i b 1-G F P$ & $\mathrm{Hyg}^{\mathrm{R}}, \mathrm{Nat}^{\mathrm{R}}$ & $\mathrm{JB} 1$ \\
\hline UNP15\#1 & a1:mfa2, bE1, bW2, $\triangle U M A G \_11651$ & $\mathrm{Phleo}^{\mathrm{R}}, \mathrm{Hyg}^{\mathrm{R}}$ & SG200 \\
\hline UNP17\#1 & $\begin{array}{l}\text { a1:mfa2, bE1, bW2, } \Delta s p p 1, \\
\text { ip }^{r}\left[P_{\text {otef:Spp1-mCherry }] i p^{s}} \text { (multiple) }\right.\end{array}$ & $\begin{array}{l}\text { Phleo }^{R}, \\
\text { Hyg }^{R}, \mathrm{Cbx}^{R}\end{array}$ & UNP6\#3 \\
\hline UNP18\#1 & 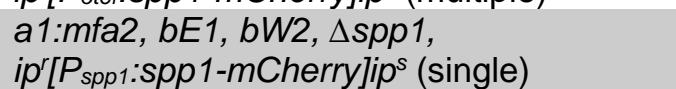 & $\begin{array}{l}\mathrm{Phleo}^{\mathrm{R}} \\
\mathrm{Hyg}^{\mathrm{R}}, \mathrm{Cbx}^{\mathrm{R}}\end{array}$ & UNP6\#3 \\
\hline UNP19\#1 & a1, $\Delta b, P_{\text {crg1: }}$ clp1, cib1-3xHA & $\begin{array}{l}\mathrm{Hyg}^{\mathrm{R}}, \quad \mathrm{Cbx}{ }^{\mathrm{R}} \\
\mathrm{Nat}^{\mathrm{R}}\end{array}$ & UV0151 \\
\hline UNP19\#2 & 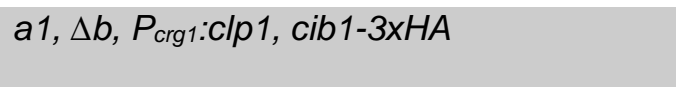 & $\begin{array}{l}\mathrm{Hyg}^{\mathrm{R}}, \quad \mathrm{Cbx}{ }^{\mathrm{R}} \text {, } \\
\mathrm{Nat}^{\mathrm{R}}\end{array}$ & UVO151 \\
\hline UNP19\#3 & 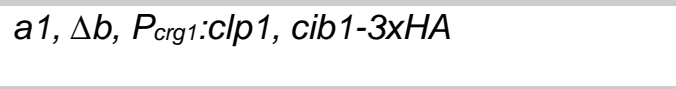 & $\begin{array}{l}\mathrm{Hyg}^{\mathrm{R}}, \quad \mathrm{Cbx} x^{\mathrm{R}} \\
\mathrm{Nat}^{\mathrm{R}}\end{array}$ & UVO151 \\
\hline UNP20\#1 & $a 1, \Delta b, c i b 1-3 x H A$ & $\mathrm{Hyg}^{\mathrm{R}}, \mathrm{Nat}^{\mathrm{R}}$ & JB1 \\
\hline UNP20\#2 & $a 1, \Delta b, c i b 1-3 x H A$ & $\mathrm{Hyg}^{\mathrm{R}}, \mathrm{Nat}^{\mathrm{R}}$ & JB1 \\
\hline UNP20\#3 & $a 1, \Delta b, c i b 1-3 x H A$ & $\mathrm{Hyg}^{\mathrm{R}}, \mathrm{Nat}^{\mathrm{R}}$ & JB1 \\
\hline UNP21\#1 & a1:mfa2, bE1, bW2, $\triangle U M A G \_00542$ & $\mathrm{Phleo}^{\mathrm{R}}, \mathrm{Hyg}^{\mathrm{R}}$ & SG200 \\
\hline UNP21\#2 & a1:mfa2, bE1, bW2, $\triangle U M A G \_00542$ & $\mathrm{Phleo}^{\mathrm{R}}, \mathrm{Hyg}^{\mathrm{R}}$ & SG200 \\
\hline UNP21\#3 & a1:mfa2, bE1, bW2, $\triangle U M A G \_00542$ & $\mathrm{Phleo}^{\mathrm{R}}, \mathrm{Hyg}^{\mathrm{R}}$ & SG200 \\
\hline UNP22\#1 & a1:mfa2, bE1, bW2, $\triangle U M A G \_05898$ & $\mathrm{Phleo}^{\mathrm{R}}, \mathrm{Hyg}^{\mathrm{R}}$ & SG200 \\
\hline UNP22\#2 & a1:mfa2, bE1, bW2, $\triangle U M A G \_05898$ & $\mathrm{Phleo}^{\mathrm{R}}, \mathrm{Hyg}^{\mathrm{R}}$ & SG200 \\
\hline UNP22\#3 & a1:mfa2, bE1, bW2, $\triangle U M A G \_05898$ & $\mathrm{Phleo}^{\mathrm{R}}, \mathrm{Hyg}^{\mathrm{R}}$ & SG200 \\
\hline UNP23\#1 & a1:mfa2, bE1, bW2, $\triangle U M A G \_10911$ & $\mathrm{Phleo}^{\mathrm{R}}, \mathrm{Hyg}^{\mathrm{R}}$ & SG200 \\
\hline UNP23\#2 & a1:mfa2, bE1, bW2, $\triangle U M A G \_10911$ & $\mathrm{Phleo}^{\mathrm{R}}, \mathrm{Hyg}^{\mathrm{R}}$ & SG200 \\
\hline UNP23\#3 & a1:mfa2, bE1, bW2, $\triangle U M A G \_10911$ & $\mathrm{Phleo}^{\mathrm{R}}, \mathrm{Hyg}^{\mathrm{R}}$ & SG200 \\
\hline UNP24\#1 & a1:mfa2, bE1, bW2, $\triangle U M A G \_11402$ & $\mathrm{Phleo}^{\mathrm{R}}, \mathrm{Hyg}^{\mathrm{R}}$ & SG200 \\
\hline UNP24\#2 & a1:mfa2, bE1, bW2, $\triangle U M A G \_11402$ & Phleo $^{R}, \mathrm{Hyg}^{\mathrm{R}}$ & SG200 \\
\hline UNP24\#3 & a1:mfa2, bE1, bW2, $\triangle U M A G \_11402$ & $\mathrm{Phleo}^{\mathrm{R}}, \mathrm{Hyg}^{\mathrm{R}}$ & SG200 \\
\hline UNP25\#1 & a1:mfa2, bE1, bW2, $\triangle U M A G \_05721$ & $\mathrm{Phleo}^{\mathrm{R}}, \mathrm{Hyg}^{\mathrm{R}}$ & SG200 \\
\hline UNP25\#2 & a1:mfa2, bE1, bW2, $\triangle U M A G \_05721$ & $\mathrm{Phleo}^{\mathrm{R}}, \mathrm{Hyg}^{\mathrm{R}}$ & SG200 \\
\hline UNP25\#3 & a1:mfa2, bE1, bW2, $\triangle U M A G \_05721$ & $\mathrm{Phleo}^{\mathrm{R}}, \mathrm{Hyg}^{\mathrm{R}}$ & SG200 \\
\hline UNP26\#1 & a1, $\Delta b, P_{\text {crg1:clp1, }} P_{\text {tef::TTA }}$, $P_{\text {teto:cib1-GFP }}$ & $\begin{array}{l}\mathrm{Hyg}^{\mathrm{R}}, \mathrm{Cbx}^{\mathrm{R}} \\
\mathrm{Nat}^{\mathrm{R}}, \mathrm{Phleo}^{\mathrm{R}}\end{array}$ & UNP13\#3 \\
\hline UNP26\#2 & a1, $\Delta b, P_{\text {crg1:clp1, }} P_{\text {tef:tTA }}{ }^{*}, P_{\text {teto:cib1-GFP }}$ & $\begin{array}{l}\mathrm{Hyg}^{\mathrm{R}}, \mathrm{Cbx}^{\mathrm{R}} \\
\mathrm{Nat}^{\mathrm{R}}, \mathrm{Phleo}^{\mathrm{R}}\end{array}$ & UNP13\#3 \\
\hline UNP26\#3 & a1, $\Delta b, P_{\text {crg1:clp1, }} P_{\text {tef::TTA }}{ }^{*} P_{\text {teto:cib1-GFP }}$ & $\begin{array}{l}\mathrm{Hyg}^{\mathrm{R}}, \mathrm{Cbx}^{\mathrm{R}} \\
\mathrm{Nat}^{\mathrm{R}}, \mathrm{Phleo}^{\mathrm{R}}\end{array}$ & UNP13\#3 \\
\hline UNP27\#1 & a1, $\Delta b, P_{\text {tef:tTA }}{ }^{\star}, P_{\text {teto:cib1-GFP }}$ & $\begin{array}{l}\text { Hyg }^{R}, \quad \text { Nat }^{R}, \\
\text { Phleo }^{R}\end{array}$ & UNP14\#2 \\
\hline UNP27\#2 & a1, $\Delta b, P_{\text {tef: }}: T A^{*}, P_{\text {teto:cib1-GFP }}$ & $\begin{array}{l}\mathrm{Hyg}^{\mathrm{R}}, \quad \mathrm{Nat}^{\mathrm{R}}, \\
\text { Phleo }^{\mathrm{R}}\end{array}$ & UNP14\#2 \\
\hline UNP27\#3 & a1, $\Delta b, P_{\text {tef: }}: T^{*}, P_{\text {teto:cib1GFP }}$ & $\begin{array}{l}\mathrm{Hyg}^{\mathrm{R}}, \quad \mathrm{Nat}^{\mathrm{R}}, \\
\text { Phleo }^{\mathrm{R}}\end{array}$ & UNP14\#2 \\
\hline UNP28\#1 & $\begin{array}{l}\text { a1:mfa2, bE1, bW2, } \\
\text { } U \text { UMAG_10911 }\end{array}$ & $\begin{array}{l}\text { Phleo }^{R} \\
\text { Hyg }^{R}, \text { Nat }^{R}\end{array}$ & UNP21\#3 \\
\hline UNP28\#2 & $\begin{array}{l}\text { a1:mfa2, bE1, bW2, } \triangle U M A G \_00542, \\
\Delta U M A G \_10911\end{array}$ & $\begin{array}{l}\text { Phleo }^{R} \\
\text { Hyg }^{R}, \text { Nat }^{R}\end{array}$ & UNP21\#3 \\
\hline
\end{tabular}




\begin{tabular}{|c|c|c|c|}
\hline UNP35\#1 & 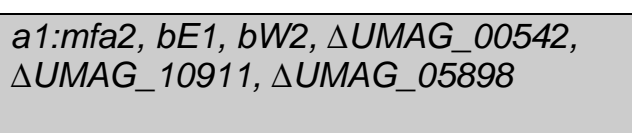 & $\begin{array}{l}\text { Phleo }^{R}, \\
\text { Hyg }^{R}, \quad N^{R} t^{R}, \\
\text { Cbx }^{R}\end{array}$ & UNP28\#1 \\
\hline UNP35\#2 & 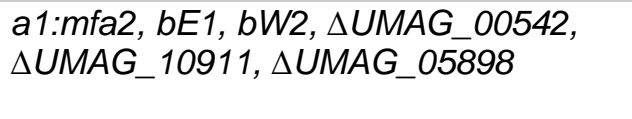 & $\begin{array}{l}\text { Phleo }^{\mathrm{R}}, \\
\mathrm{Hyg}^{\mathrm{R}}, \quad \mathrm{Nat}^{\mathrm{R}}, \\
\mathrm{Cbx}^{\mathrm{R}}\end{array}$ & UNP28\#1 \\
\hline UNP35\#3 & 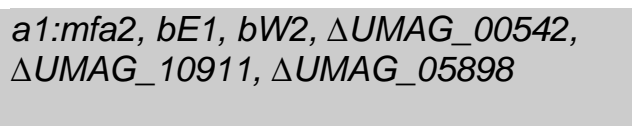 & $\begin{array}{l}\text { Phleo }^{\mathrm{R}}, \\
\text { Hyg }^{\mathrm{R}}, \quad \mathrm{Nat}^{\mathrm{R}} \text {, } \\
\text { Cbx }^{\mathrm{R}}\end{array}$ & UNP28\#1 \\
\hline UNP36\#1 & $\begin{array}{l}\text { a1:mfa2, bE1, bW2, } \\
\text { AUMAG_10911, } U \text { UMAG_11402 }\end{array}$ & $\begin{array}{l}\text { Phleo }^{\mathrm{R}}, \\
\mathrm{Hyg}^{\mathrm{R}}, \quad \mathrm{Nat}^{\mathrm{R}} \text {, } \\
\mathrm{Cbx}^{\mathrm{R}}\end{array}$ & UNP28\#1 \\
\hline UNP36\#2 & 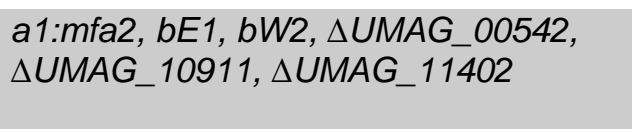 & $\begin{array}{l}\text { Phleo }^{R}, \\
\text { Hyg }^{R}, \quad \text { Nat }^{R} \text {, } \\
\text { Cbx }^{R}\end{array}$ & UNP28\#1 \\
\hline UNP36\#3 & $\begin{array}{l}\text { a1:mfa2, bE1, bW2, } \triangle U M A G \_00542, \\
\Delta U M A G \_10911, \Delta U M A G \_11402\end{array}$ & $\begin{array}{l}\mathrm{Phleo}^{\mathrm{R}}, \\
\mathrm{Hyg}^{\mathrm{R}}, \quad \mathrm{Nat}^{\mathrm{R}}, \\
\mathrm{Cbx}^{\mathrm{R}}\end{array}$ & UNP28\#1 \\
\hline UNP40\#1 & a1, $\Delta b, i p^{R}\left[P_{\text {otef: }}\right.$ eGFP-3xHA]ip $p^{S}$ (single) & $\mathrm{Hyg}^{\mathrm{R}}, \mathrm{Cbx}^{\mathrm{R}}$ & JB1 \\
\hline UNP43\#1 & a1:mfa2, bE1, bW2, $\triangle U M A G \_01025$ & $\mathrm{Phleo}^{\mathrm{R}}, \mathrm{Hyg}^{\mathrm{R}}$ & SG200 \\
\hline UNP44\#1 & a1:mfa2, bE1, bW2, $\triangle U M A G \_12304$ & $\mathrm{Phleo}^{\mathrm{R}}, \mathrm{Hyg}^{\mathrm{R}}$ & SG200 \\
\hline UNP45\#1 & a1:mfa2, bE1, bW2, $\triangle U M A G \_12332$ & $\mathrm{Phleo}^{\mathrm{R}}, \mathrm{Hyg}^{\mathrm{R}}$ & SG200 \\
\hline UNP47\#1 & a1:mfa2, bE1, bW2, cib1 ${ }^{\text {S468A-GFP }}$ & $\mathrm{Phleo}^{\mathrm{R}}, \mathrm{Nat}^{\mathrm{R}}$ & SG200 \\
\hline UNP47\#2 & a1:mfa2, bE1, bW2, cib1 ${ }^{\text {S468A-GFP }}$ & $\mathrm{Phleo}^{\mathrm{R}}, \mathrm{Nat}^{\mathrm{R}}$ & SG200 \\
\hline UNP47\#3 & a1:mfa2, bE1, bW2, cib1 ${ }^{\mathrm{S} 468 \mathrm{~A}}$-GFP & $\mathrm{Phleo}^{\mathrm{R}}, \mathrm{Nat}^{\mathrm{R}}$ & SG200 \\
\hline UNP50\#1 & a1:mfa2, bE1, bW2, cib1 ${ }^{\text {S468E }}$-GFP & $\mathrm{Phleo}^{\mathrm{R}}, \mathrm{Nat}^{\mathrm{R}}$ & SG200 \\
\hline UNP50\#2 & a1:mfa2, bE1, bW2, cib1 ${ }^{\text {S468E-GFP }}$ & $\mathrm{Phleo}^{\mathrm{R}}, \mathrm{Nat}^{\mathrm{R}}$ & SG200 \\
\hline UNP50\#3 & a1:mfa2, bE1, bW2, cib1 ${ }^{S 468 E_{-G F P}}$ & $\mathrm{Phleo}^{\mathrm{R}}, \mathrm{Nat}^{\mathrm{R}}$ & SG200 \\
\hline UNP59\#1 & a1:mfa2, bE1, bW2, $\triangle U M A G \_02487$ & $\mathrm{Phleo}^{\mathrm{R}}, \mathrm{Hyg}^{\mathrm{R}}$ & SG200 \\
\hline UNP60\#1 & a1:mfa2, bE1, bW2, $\triangle U M A G \_04605$ & $\mathrm{Phleo}^{\mathrm{R}}, \mathrm{Hyg}^{\mathrm{R}}$ & SG200 \\
\hline UNP61\#1 & a1:mfa2, bE1, bW2, $\triangle U M A G \_10006$ & $\mathrm{Phleo}^{\mathrm{R}}, \mathrm{Hyg}^{\mathrm{R}}$ & SG200 \\
\hline UNP62\#1 & a1:mfa2, bE1, bW2, $\triangle U M A G \_12318$ & $\mathrm{Phleo}^{\mathrm{R}}, \mathrm{Hyg}^{\mathrm{R}}$ & SG200 \\
\hline UNP63\#1 & a1:mfa2, bE1, bW2, $\triangle U M A G \_03541$ & $\mathrm{Phleo}^{\mathrm{R}}, \mathrm{Hyg}^{\mathrm{R}}$ & SG200 \\
\hline UNP64\#1 & a1:mfa2, bE1, bW2, cib1 ${ }^{\text {T381E }}$-GFP & $\mathrm{Phleo}^{\mathrm{R}}, \mathrm{Nat}^{\mathrm{R}}$ & SG200 \\
\hline UNP64\#2 & a1:mfa2, bE1, bW2, cib1 ${ }^{\text {T381E }}$-GFP & $\mathrm{Phleo}^{\mathrm{R}}, \mathrm{Nat}^{\mathrm{R}}$ & SG200 \\
\hline UNP64\#3 & a1:mfa2, bE1, bW2, cib1 ${ }^{\text {T381E }}$-GFP & $\mathrm{Phleo}^{\mathrm{R}}, \mathrm{Nat}^{\mathrm{R}}$ & SG200 \\
\hline UNP67\#1 & a1:mfa2, bE1, bW2, cib1 ${ }^{T 381 A}-G F P$ & $\mathrm{Phleo}^{\mathrm{R}}, \mathrm{Nat}^{\mathrm{R}}$ & SG200 \\
\hline UNP67\#2 & a1:mfa2, bE1, bW2, cib1 ${ }^{T 381 A}-G F P$ & $\mathrm{Phleo}^{\mathrm{R}}, \mathrm{Nat}^{\mathrm{R}}$ & SG200 \\
\hline UNP67\#3 & a1:mfa2, bE1, bW2, cib1 ${ }^{T 381 A}-G F P$ & $\mathrm{Phleo}^{\mathrm{R}}, \mathrm{Nat}^{\mathrm{R}}$ & SG200 \\
\hline UNP70\#1 & a1:mfa2, bE1, bW2, $\triangle U M A G \_02578$ & Phleo $^{\mathrm{R}}, \mathrm{Hyg}^{\mathrm{R}}$ & SG200 \\
\hline UNP70\#3 & a1:mfa2, bE1, bW2, $\triangle U M A G \_02578$ & $\mathrm{Phleo}^{\mathrm{R}}, \mathrm{Hyg}^{\mathrm{R}}$ & SG200 \\
\hline UNP70\#5 & a1:mfa2, bE1, bW2, $\triangle U M A G \_02578$ & $\mathrm{Phleo}^{\mathrm{R}}, \mathrm{Hyg}^{\mathrm{R}}$ & SG200 \\
\hline UNP72\#1 & a1:mfa2, bE1, bW2, cib1 ${ }^{T 381 A, S 468 A}$-GFP & $\mathrm{Phleo}^{\mathrm{R}}, \mathrm{Nat}^{\mathrm{R}}$ & SG200 \\
\hline UNP72\#2 & a1:mfa2, bE1, bW2, cib1 $1^{T 381 A, S 468 A}-G F P$ & $\mathrm{Phleo}^{\mathrm{R}}, \mathrm{Nat}^{\mathrm{R}}$ & SG200 \\
\hline UNP72\#3 & a1:mfa2, bE1, bW2, cib1 ${ }^{\text {T381A,S468A-GFP }}$ & $\mathrm{Phleo}^{\mathrm{R}}, \mathrm{Nat}^{\mathrm{R}}$ & SG200 \\
\hline UNP75\#1 & a1:mfa2, bE1, bW2, cib1 ${ }^{\text {T381E,S468E-GFP }}$ & $\mathrm{Phleo}^{\mathrm{R}}, \mathrm{Nat}^{\mathrm{R}}$ & SG200 \\
\hline
\end{tabular}




\begin{tabular}{|c|c|c|c|}
\hline UNP75\#2 & a1:mfa2, bE1, bW2, cib1 ${ }^{T 381 E, S 468 E_{-G F P}}$ & $\mathrm{Phleo}^{\mathrm{R}}, \mathrm{Nat}^{\mathrm{R}}$ & SG200 \\
\hline UNP75\#3 & a1:mfa2, bE1, bW2, cib1 ${ }^{T 381 E, S 468 E_{-G F P}}$ & $\mathrm{Phleo}^{\mathrm{R}}, \mathrm{Nat}^{\mathrm{R}}$ & SG200 \\
\hline UNP78\#2 & a1:mfa2, bE1, bW2, $\triangle U M A G \_10921$ & $\mathrm{Phleo}^{\mathrm{R}}, \mathrm{Hyg}^{\mathrm{R}}$ & SG200 \\
\hline UNP79\#1 & a1:mfa2, bE1, bW2, $\triangle U M A G \_11083$ & $\mathrm{Phleo}^{\mathrm{R}}, \mathrm{Hyg}^{\mathrm{R}}$ & SG200 \\
\hline UNP82\#1 & a1:mfa2, bE1, bW2, $\triangle U M A G \_00258$ & $\mathrm{Phleo}^{\mathrm{R}}, \mathrm{Hyg}^{\mathrm{R}}$ & SG200 \\
\hline UNP83\#1 & a1:mfa2, bE1, bW2, $\triangle U M A G \_10686$ & $\mathrm{Phleo}^{\mathrm{R}}, \mathrm{Hyg}^{\mathrm{R}}$ & SG200 \\
\hline UNP84\#1 & a1:mfa2, bE1, bW2, $\triangle U M A G \_11190$ & $\mathrm{Phleo}^{\mathrm{R}}, \mathrm{Hyg}^{\mathrm{R}}$ & SG200 \\
\hline UNP85\#1 & a1:mfa2, bE1, bW2, $\triangle U M A G \_03665$ & $\mathrm{Phleo}^{\mathrm{R}}, \mathrm{Hyg}^{\mathrm{R}}$ & SG200 \\
\hline UNP86\#1 & a1:mfa2, bE1, bW2, $\triangle U M A G \_11763$ & $\mathrm{Phleo}^{\mathrm{R}}, \mathrm{Hyg}^{\mathrm{R}}$ & SG200 \\
\hline UNP87\#1 & a1:mfa2, bE1, bW2, $\triangle U M A G \_04896$ & $\mathrm{Phleo}^{\mathrm{R}}, \mathrm{Hyg}^{\mathrm{R}}$ & SG200 \\
\hline UNP89\#1 & a1:mfa2, bE1, bW2, $\triangle U M A G \_01112$ & $\mathrm{Phleo}^{\mathrm{R}}, \mathrm{Hyg}^{\mathrm{R}}$ & SG200 \\
\hline UNP90\#1 & a1:mfa2, bE1, bW2, $\triangle U M A G \_01232$ & $\mathrm{Phleo}^{\mathrm{R}}, \mathrm{Hyg}^{\mathrm{R}}$ & SG200 \\
\hline UNP91\#1 & $\begin{array}{l}\text { a1:mfa2, bE1, bW2, } \Delta s p p 1 \\
\text { ip }\left[P_{\text {otef:HM13-mCherry]ip }} \text { (single) }\right.\end{array}$ & $\begin{array}{l}\text { Phleo }^{R} \\
\text { Hyg }^{R}, \mathrm{Cbx}^{\mathrm{R}}\end{array}$ & UNP6\#1 \\
\hline UNP91\#2 & $\begin{array}{l}\text { a1:mfa2, bE1, bW2, } \Delta s p p 1, \\
\text { ip } p^{r}\left[P_{\text {otef: }} \text { HM13-mCherry]ips }\right.\end{array}$ & $\begin{array}{l}\text { Phleo }^{R} \\
\text { Hyg }^{R}, \text { Cbx }^{R}\end{array}$ & UNP6\#1 \\
\hline UNP92\#1 & 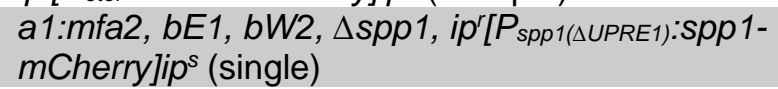 & $\begin{array}{l}\text { Phleo }^{R} \\
\text { Hyg }^{R}, \mathrm{Cbx}^{\mathrm{R}}\end{array}$ & UNP6\#3 \\
\hline UNP92\#2 & $\begin{array}{l}\text { a1:mfa2, bE1, bW2, } \Delta s p p 1, i p^{r}\left[P_{\text {spp1(AUPRE1):spp1- }}\right. \\
\text { mCherry]ip }{ }^{s} \text { (single) }\end{array}$ & $\begin{array}{l}\text { Phleo }^{R} \\
\text { Hyg }^{R}, \mathrm{Cbx}^{\mathrm{R}}\end{array}$ & UNP6\#3 \\
\hline UNP92\#3 & $\begin{array}{l}\text { a1:mfa2, bE1, bW2, } \triangle \text { spp1, ip }{ }^{r}\left[P_{\text {spp1(AUPRE1):Spp1- }}\right. \\
\text { mCherry }] p^{s} \text { (single) }\end{array}$ & $\begin{array}{l}\text { Phleo }^{R} \\
\text { Hyg }^{R}, \mathrm{Cbx}^{\mathrm{R}}\end{array}$ & UNP6\#3 \\
\hline UNP93\#1 & 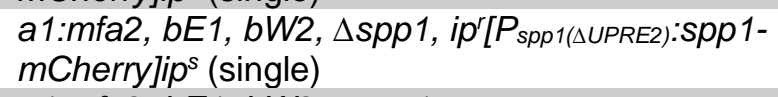 & $\begin{array}{l}\text { Phleo }^{R} \\
\text { Hyg }^{R}, \mathrm{Cbx}^{\mathrm{R}}\end{array}$ & UNP6\#3 \\
\hline UNP94\#1 & $\begin{array}{l}\text { a1:mfa2, bE1, bW2, } \triangle \text { spp1, } \\
\text { ip }^{\prime}\left[P_{\text {Spp } 1(\triangle U P R E 1, \Delta U P R E 2): s p p 1-m C h e r r y}\right] i p^{s} \text { (single) }\end{array}$ & $\begin{array}{l}\text { Phleo }^{R} \\
\text { Hyg }^{R}, \mathrm{Cbx}^{\mathrm{R}}\end{array}$ & UNP6\#3 \\
\hline UNP94\#2 & 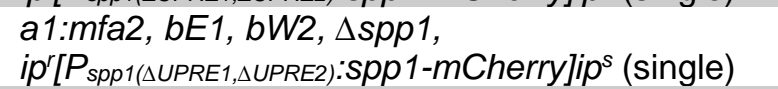 & $\begin{array}{l}\text { Phleo }^{R} \\
\text { Hyg }^{R}, \mathrm{Cbx}^{R}\end{array}$ & UNP6\#3 \\
\hline UNP94\#3 & 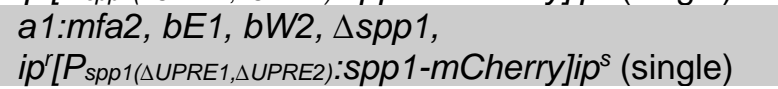 & $\begin{array}{l}\text { Phleo }^{R} \\
\text { Hyg }^{R}, \mathrm{Cbx}^{R}\end{array}$ & UNP6\#3 \\
\hline UNP98\#1 & $\begin{array}{l}\text { a1:mfa2, bE1, bW2, } \\
\text { ip }^{\prime}\left[P_{\text {otef:pep1-mCherry }] i p^{s}} \text { (single) }\right.\end{array}$ & $\mathrm{Phleo}^{\mathrm{R}}, \mathrm{Cbx}^{\mathrm{R}}$ & SG200 \\
\hline UNP99\#1 & $\begin{array}{l}\text { a1:mfa2, bE1, bW2, } \Delta \text { spp1, } \\
\text { ip }\left[P_{\text {otet:pep1-mCherry }] i p^{s} \text { (single) }}\right.\end{array}$ & $\begin{array}{l}\text { Phleo }^{R} \\
\text { Hyg }^{R}, \mathrm{Cbx}^{R}\end{array}$ & UNP6\#3 \\
\hline UNP100\#2 & $\begin{array}{l}\text { a1:mfa2, bE1, bW2, } \\
\text { ip }^{\prime}\left[P_{\text {otef:tin2-mCherry }] p^{s}} \text { (multiple) }\right.\end{array}$ & Phleo $^{R}, \mathrm{Cbx}^{\mathrm{R}}$ & SG200 \\
\hline UNP101\#1 & $\begin{array}{l}\text { a1:mfa2, bE1, bW2, } \Delta s p p 1, \\
\text { ip }^{\prime}\left[P_{\text {otef:tin2-mCherry }] i p^{s}} \text { (multiple) }\right.\end{array}$ & $\begin{array}{l}\text { Phleo }^{R} \\
\text { Hyg }^{R}, \mathrm{Cbx}^{R}\end{array}$ & UNP6\#3 \\
\hline UNP102\#1 & $\begin{array}{l}\text { a1:mfa2, bE1, bW2, } \\
\text { ip }\left[P_{\text {otef:Cmu1-mCherry }] i p^{s} \text { (double) }}\right.\end{array}$ & $\mathrm{Phleo}^{\mathrm{R}}, \mathrm{Cbx}^{\mathrm{R}}$ & SG200 \\
\hline UNP103\#2 & $\begin{array}{l}\text { a1:mfa2, bE1, bW2, } \Delta \text { spp1, } \\
\text { ip }\left[P_{\text {otef:Cmu1-mCherry }] i p^{s} \text { (double) }}\right.\end{array}$ & $\begin{array}{l}\text { Phleo }^{R} \\
\text { Hyg }^{R}, \mathrm{Cbx}^{R}\end{array}$ & UNP6\#3 \\
\hline UNP104\#1 & $\begin{array}{l}\text { a1:mfa2, bE1, bW2, } \Delta s p p 1, \\
\text { ip'[Potef:Spp1-mCherry]ip }{ }^{s}, \Delta c i b 1\end{array}$ & $\begin{array}{l}\text { Phleo }^{R}, \\
\text { Hyg }^{R}, C^{R} x^{R}, \\
\text { Nat }^{R}\end{array}$ & UNP17\#1 \\
\hline UNP105\#1 & $\begin{array}{l}\text { a1:mfa2, bE1, bW2, } \Delta s p p 1 \text {, } \\
\text { ip }\left[P_{\text {spp1: }}: \text { spp1-mCherry]ip }{ }^{s}, \Delta c i b 1\right.\end{array}$ & $\begin{array}{l}\text { Phleo }^{\mathrm{R}}, \\
\text { Hygg }^{\mathrm{R}}, \mathrm{Cbx}^{\mathrm{R}} \text {, } \\
\text { Nat }^{\mathrm{R}}\end{array}$ & UNP18\#1 \\
\hline
\end{tabular}


Table 5.3: Ustilago maydis strains generated in a supervised master thesis (Hach, 2018)

\begin{tabular}{|c|c|c|c|}
\hline Strain & Genotype & Resistance & Source \\
\hline UCH002\#1 & $\begin{array}{l}\text { a1:mfa2, bE1, bW2, } \Delta s p p 1, \\
\text { ip }^{\prime}\left[P_{\text {otef:Spp1 }}{ }^{D 279 A}-m \text { Cherry]ip }\right. \\
\text { (single) }\end{array}$ & $\begin{array}{l}\text { Hyg }^{\mathrm{R}} \text {, } \\
\text { Phleo }^{\mathrm{R}}, \mathrm{Cbx}^{\mathrm{R}}\end{array}$ & UNP6\#3 \\
\hline UCH003\#1 & $\begin{array}{l}\text { a1:mfa2, bE1, bW2, } \Delta \text { spp1, } \\
\text { ip }\left[\text { p }^{r} \text { otef:YPF1-mCherry]ip }\right. \\
\text { (multiple) }\end{array}$ & $\begin{array}{l}\text { Hyg }^{\mathrm{R}} \text {, } \\
\text { Phleo }^{\mathrm{R}}, \mathrm{Cbx}^{\mathrm{R}}\end{array}$ & UNP6\#3 \\
\hline UCH004\#1 & $\begin{array}{l}\text { a1:mfa2, bE1, bW2, } \Delta s p p 1 \text {, } \\
\text { ip }\left[p_{\text {otef:SppA-mCherry }] i p^{s} \text { (single) }}\right.\end{array}$ & $\begin{array}{l}\mathrm{Hyg}^{\mathrm{R}} \text {, } \\
\text { Phleo }^{\mathrm{R}}, \mathrm{Cbx}^{\mathrm{R}}\end{array}$ & UNP6\#3 \\
\hline UCH006\#1 & $\begin{array}{l}\text { a1:mfa2, bE1, bW2, } \Delta \text { spp1, } \\
\text { ip } p^{r}\left[P_{\text {spp1:Spp1 }}{ }^{D 279 A}-m C h e r r y\right] i p^{s} \text { (multiple) }\end{array}$ & $\begin{array}{l}\text { Hyg }^{\mathrm{R}} \text {, } \\
\text { Phleo }^{\mathrm{R}}, \mathrm{Cbx}^{\mathrm{R}}\end{array}$ & UNP6\#3 \\
\hline UCH007\#1 & $\begin{array}{l}\text { a1:mfa2, bE1, bW2, } \Delta \text { spp1, } \\
\text { ip }\left[P_{\text {otef:Spp1-GFP }] i p^{s} \text { (single) }}\right.\end{array}$ & $\begin{array}{l}\text { Hyg }^{\mathrm{R}} \text {, } \\
\text { Phleo }^{\mathrm{R}}, \mathrm{Cbx}^{\mathrm{R}}\end{array}$ & UNP6\#3 \\
\hline UCH008\#1 & $\begin{array}{l}\text { a1:mfa2, bE1, bW2, } \Delta \text { spp1, } \\
\text { ip }\left[\text { p }^{r} \text { otef:UhSpp1-mCherry]ip }{ }^{s} \text { (single) }\right.\end{array}$ & $\begin{array}{l}\text { Hyg }^{\mathrm{R}} \text {, } \\
\text { Phleo }^{\mathrm{R}}, \mathrm{Cbx}^{\mathrm{R}}\end{array}$ & UNP6\#3 \\
\hline UCH009\#1 & $\begin{array}{l}\text { a1:mfa2, bE1, bW2, } \Delta \text { spp1, } \\
\text { ip'[Potef:SrSpp1-mCherry]ips (single) }\end{array}$ & $\begin{array}{l}\mathrm{Hyg}^{\mathrm{R}} \text {, } \\
\text { Phleo }^{\mathrm{R}}, \mathrm{Cbx}^{\mathrm{R}}\end{array}$ & UNP6\#3 \\
\hline UCH010\#1 & a1:mfa2, bE1, bW2, $\triangle U M A G \_05009$ & $\mathrm{Hyg}^{\mathrm{R},} \mathrm{Phleo}^{\mathrm{R}}$ & SG200 \\
\hline UCH011\#1 & a1:mfa2, bE1, bW2, $\triangle U M A G \_03507$ & $\mathrm{Hyg}^{\mathrm{R},} \mathrm{Phleo}^{\mathrm{R}}$ & SG200 \\
\hline UCH012\#1 & a1:mfa2, bE1, bW2, $\triangle U M A G \_11513$ & $\mathrm{Hyg}^{\mathrm{R},} \mathrm{Phleo}^{\mathrm{R}}$ & SG200 \\
\hline UCH013\#1 & a1:mfa2, bE1, bW2, $\triangle U M A G \_02944$ & $\mathrm{Hyg}^{\mathrm{R},} \mathrm{Phleo}^{\mathrm{R}}$ & SG200 \\
\hline UCH014\#1 & $\begin{array}{l}\text { a1:mfa2, bE1, bW2, } \Delta s p p 1, \\
\text { ip }^{r}\left[P_{\text {otef:Spp1 }}{ }^{D 279 A}-G F P\right] i p^{s} \text { (single) }\end{array}$ & $\begin{array}{l}\mathrm{Hyg}^{\mathrm{R}} \text {, } \\
\text { Phleo }^{\mathrm{R}}, \mathrm{Cbx}^{\mathrm{R}}\end{array}$ & UNP6\#3 \\
\hline UCH015\#1 & a1:mfa2, bE1, bW2, $\triangle U M A G \_03404$ & $\mathrm{Hyg}^{\mathrm{R},} \mathrm{Phleo}^{\mathrm{R}}$ & SG200 \\
\hline UCH016\#1 & a1:mfa2, bE1, bW2, $\triangle U M A G \_12178$ & $\mathrm{Hyg}^{\mathrm{R},} \mathrm{Phleo}^{\mathrm{R}}$ & SG200 \\
\hline UCH017\#1 & $\begin{array}{l}\text { a1:mfa2, bE1, bW2, } \Delta \text { pit2, } \\
\text { ip'[Potef:pit2-mCherry (\#1)]ips, } \Delta \text { spp1 }\end{array}$ & $\begin{array}{l}\mathrm{Hyg}^{\mathrm{R}} \text {, } \\
\text { Phleo }^{\mathrm{R}} \\
\mathrm{Cbx}^{\mathrm{R}}, \mathrm{Neo}^{\mathrm{R}}\end{array}$ & UMH243 \\
\hline UCH020\#1 & $\begin{array}{l}\text { a1:mfa2, bE1, bW2, } \Delta s p p 1, \\
\text { ip }\left[p^{r}\left[P_{\text {otef: }}: H M 13-m C h e r r y\right] i p^{s} \text { (single) }\right.\end{array}$ & $\begin{array}{l}\mathrm{Hyg}^{\mathrm{R}} \text {, } \\
\text { Phleo }^{\mathrm{R}}, \mathrm{Cbx}^{\mathrm{R}}\end{array}$ & UNP6\#3 \\
\hline UCH021\#1 & $\begin{array}{l}\text { a1:mfa2, bE1, bW2, } \\
\text { ip } p^{r}\left[P_{\text {otef:UMAG_02578-GFP }] i p^{s}}\right.\end{array}$ & $\begin{array}{l}\mathrm{Hyg}^{\mathrm{R}} \text {, } \\
\text { Phleo }^{\mathrm{R}}, \mathrm{Cbx}^{\mathrm{R}}\end{array}$ & SG200 \\
\hline UCH021\#2 & $\begin{array}{l}\text { a1:mfa2, bE1, bW2, } \\
\text { ip }\left[P_{\text {otef:UMAG_02578-GFP }] i p^{s}}\right.\end{array}$ & $\begin{array}{l}\text { Hyg }^{\mathrm{R}} \text {, } \\
\text { Phleo }^{\mathrm{R}}, \mathrm{Cbx}^{\mathrm{R}}\end{array}$ & SG200 \\
\hline UCH021\#3 & $\begin{array}{l}\text { a1:mfa2, bE1, bW2, } \\
\text { ip }\left[p^{r}\left[P_{\text {otef:UMAG_U2578-GFP }] i p^{s}}\right.\right.\end{array}$ & $\begin{array}{l}\mathrm{Hyg}^{\mathrm{R}} \text {, } \\
\text { Phleo }^{\mathrm{R}}, \mathrm{Cbx}^{\mathrm{R}}\end{array}$ & SG200 \\
\hline
\end{tabular}

\subsubsection{Chemicals}

All chemicals used in this work were p.a. quality and were obtained from Ambion, Amersham, BioRad, Carl Roth, chromotek, Difco, Duchefa, Fluka, Formedium, Gerbu, Invitrogen, Merck, Millipore, New England Biolabs, Pharmacia, Promega, Riedel-de-Han, Roche, Seakem, Serva, Sigma-Aldrich and Thermo Fisher Scientific. For further details see Brachmann et al. (2001).

\subsubsection{Kits}

In this work the kits QIAquick Gel Extraction Kit for the isolation of DNA fragments from agarose gels, QIAquick PCR Purification Kit (Qiagen) for the purification of DNA fragments and plasmids, TOPO TA Cloning ${ }^{\circledR}$ Kit (Invitrogen) for the cloning of DNA fragments, TurboDNase Kit (Ambion) for DNase 
digestion of genomic DNA in RNA samples for qRT-PCR and RNAseq analysis, MesaGreen qPCR MasterMix for SYBR Assay Kit (Eurogentech) for qRT-PCR, RevertAid First Strand cDNA Synthesis Kit (Thermo Fisher Scientific) for cDNA syntheses, RNeasy Mini Kit (Qiagen) for purification of RNA samples for RNAseq were used. For western-blotting with the Trans-Blot Turbo system (Bio-Rad), the Trans-Blot Turbo RTA Midi PVDF Transfer Kit was used.

\subsubsection{Enzymes and antibodies}

Table 5.4: Enzymes used in this work

\begin{tabular}{ll}
\hline Name & Company, catalog \# \\
\hline Restriction enzymes & Thermo Fisher Scientific \\
\hline T4 DNA Ligase & Thermo Fisher Scientific, EL0014 \\
\hline FastAP Thermosensitive Alkaline Phosphatase & Thermo Fisher Scientific, EF0654 \\
\hline Lambda Protein Phosphatase & New England Biolabs, P0753S \\
\hline Klenow Fragment, LC & Thermo Fisher Scientific, EP0054 \\
\hline Taq DNA Polymerase & New England Biolabs, M0273S \\
\hline Phusion DNA polymerase & Thermo Fisher Scientific, F-530XL \\
Phusion DNA polymerase & own production \\
\hline Q5 High-Fidelity DNA Polymerase & New England Biolabs, M0491L \\
Lysozyme (from chicken egg white) & Serva, 28263.02 \\
\hline Lysing enzyme (from Trichoderma harzianum) & Sigma-Aldrich, L1412 \\
\hline
\end{tabular}

Table 5.5: Antibodies used in this work

\begin{tabular}{lll}
\hline Name (produced in) & Concentration & Company, catalog \# \\
\hline RFP antibody (mouse) & $1: 1000$ & chromotek, 6G6 \\
Anti-GFP, N-terminal antibody (rabbit) & $1: 4000$ & Millipore, G1544 \\
Monoclonal Anti-HA antibody (mouse) & $1: 4000$ & Sigma-Aldrich, H9658 \\
Anti-Mouse IgG (H+L), HRP Conjugate (goat) & $1: 4000$ & Promega, W402B \\
Anti-Rabbit IgG (H+L), HRP Conjugate (goat) & $1: 4000$ & Promega, W401B \\
Anti-Digoxigenin-AP, Fab fragments (sheep) & $1: 10000$ & Roche, 11093274910 \\
GFP-Trap Magnetic Agarose & - & chromotek, gtma \\
Monoclonal Anti-HA-Agarose antibody (mouse) & - & Sigma-Aldrich, A2095 \\
\hline
\end{tabular}




\subsubsection{Nucleic acids}

For gel electrophoresis the DNA size standard "GeneRuler DNA Ladder Mix (Thermo Fisher Scientific)" was used.

The oligonucleotides were synthesized by Eurofins Genomics Germany GmbH. The nucleotide sequences are indicated from the 5 'end to the 3 ' end.

Table 5.6: In this work used oligonucleotides

\begin{tabular}{|c|c|}
\hline Name & Sequence 5' -> 3' \\
\hline \multicolumn{2}{|c|}{ Fusion of Cib1 with GFP or 3xHA } \\
\hline CibGFP_Lb_sense & САATCСTCAAATGAAGGCGTTCGC \\
\hline CibGFP_Lb_as & GTGGGCCGCGTTGGCCGCAGCGACGATTGAGGCCATCAGAC \\
\hline CibGFP_Rb_sense & CACGGCCTGAGTGGCCTGTTGAACACGTGCGTCAGTCCC \\
\hline CibGFP_Rb_as & CTCGCCACCTGTAGACAAACAAG \\
\hline \multicolumn{2}{|c|}{ Promotor replacement with tetO } \\
\hline 11782_LB_KIN_for & GTTCGCTGTCTGTTGATCTTTCC \\
\hline 11782_LB_KIN_rev & GTGGGCCATCTAGGCCGATGAGAGACGAACGTGAAGATC \\
\hline 11782_RB_KIN_for & CACGGCCTGAGTGGCCATGACTAGCACCACCACGTC \\
\hline 11782_RB_KIN_rev & GGTGCAGAAACACTGATCTGG \\
\hline \multicolumn{2}{|c|}{ Cib1 phosphosite mutation } \\
\hline cib1_S468A_for & TCCAGCTTCTCACGGCTCCTCTGTTGGCC \\
\hline cib1_S468A_rev & GGCCAACAGAGGAGCCGTGAGAAGCTGGA \\
\hline cib1_S468E_for & CAGCTTCTCACGGAGCCTCTGTTGGCC \\
\hline cib1_S468E_rev & GGCCAACAGAGGCTCCGTGAGAAGCTG \\
\hline cib1_T381A_for & CCGACGTCTGCCGCCCC GTCTGAACCT \\
\hline cib1_T381A_rev & AGGTTCAGACGGGGCGG CAGACGTCGG \\
\hline cib1_T381E_for & CCGACGTCTGCCGAACC GTCTGAACCT \\
\hline cib1_T381E_rev & AGGTTCAGACGGTTCGG CAGACGTCGG \\
\hline \multicolumn{2}{|l|}{ Gene deletions } \\
\hline 00258_LB_KO_for & GATCCGGTGATTGCGTCAGAA \\
\hline 00258_LB_KO_rev & GTGGGCCATCTAGGCCGATGACTCATGAGCACGCAG \\
\hline 00258_RB_KO_for & CACGGCCTGAGTGGCCAGACTCCGTAACAACACAAGAGG \\
\hline 00258_RB_KO_rev & CGAGCTATGGAGGACGGG \\
\hline 00481_LB_KO_for & GGGCCAGAATGACGACGGAG \\
\hline 00481_LB_KO_rev & CACGGCCATCTAGGCCCCACACACGAACCCCACCC \\
\hline 00481_RB_KO_for & CACGGCCTGAGTGGCCGCTGCTCGATCCAGCGATGG \\
\hline 00481_RB_KO_rev & GATCTGCGTCGCTGTCGTCG \\
\hline 00542_LB_KO_for & CGAGCGGAAGAGCACATCTTG \\
\hline 00542_LB_KO_rev & CACGGCCTGAGTGGCCCTTTGTCAAAACGTTTGAGGATCAGC \\
\hline 00542_RB_KO_for & CACGGCCATCTAGGCCCTCTCTCCСАCСTCACTGCA \\
\hline 00542_RB_KO_rev & CGAATGGAAGCCСTCTGGGA \\
\hline 01025_LB_KO_for & GAGGAACAGGGAACCACACAG \\
\hline 01025_LB_KO_rev & CACGGCCTGAGTGGCCGGTAAAGGAAGAAGTGCCGACG \\
\hline 01025_RB_KO_for & CACGGCCATCTAGGCCGCTGTTGCTTTTTCGATCCGGATC \\
\hline 01025_RB_KO_rev & CAATATGGACCGAACCGGGTG \\
\hline 01112_LB_KO_for & CAATCCCTTGTATCTCTGGGC \\
\hline 01112_LB_KO_rev & GTGGGCCTGAGTGGCCCAAGACGCGACGTCAATCTTC \\
\hline 01112_RB_KO_for & CACGGCCATCTAGGCCGACGATTCTCAAGAGTCACAAGAC \\
\hline
\end{tabular}




\begin{tabular}{|c|c|}
\hline 01112_RB_KO_rev & CTCTTGTCTTCGTTCTGCTTGC \\
\hline 01232_LB_KO_for & CGAACCGAGGTAAAGGTGGAG \\
\hline 01232_LB_KO_rev & GTGGGCCATCTAGGCCCGCGTTCTTCCACTCCACTC \\
\hline 01232_RB_KO_for & CACGGCCTGAGTGGCCGGCTGTCTTTCGCGCGTG \\
\hline 01232_RB_KO_rev & CGCGTGTGATTCGTATTTGCTAC \\
\hline 02487_LB_KO_for & GTTCACTTTGGCCGATCCTCA \\
\hline 02487_LB_KO_rev & GTGGGCCTGAGTGGCCGCTGTGCGCCGGATCTG \\
\hline 02487_RB_KO_for & CACGGCCATCTAGGCCGATAGATATATCCTTACACAGAACC \\
\hline 02487 RB KO rev & CTGTGCGTAGAGTGTTGATGG \\
\hline 02729_LB_KO_for & CGACTCACGACTCСССТTTTCAAA \\
\hline 02729_LB_KO_rev & CACGGCCTGAGTGGCCGATTGCGGAGTTCTGGAACAGAGC \\
\hline 02729_RB_KO_for & CACGGCCATCTAGGCCGCTGTAATTTGTCATCTTTCCTCGA \\
\hline 02729_RB_KO_rev & GATGCCCGAACTTGTCAACACA \\
\hline 02944_LB_KO_for & CTGAGTCAAAGGCGAAAGAGG \\
\hline 02944_LB_KO_rev & GTGGGCCTGAGTGGCCCTTGGGCTTAGAAGCAATCCAC \\
\hline 02944_RB_KO_for & CACGGCCATCTAGGCCGAAGGCATCTGCACGTCAATC \\
\hline 02944_RB_KO_rev & GGTTTGTTCAGAGGTTGGAGC \\
\hline 03404_LB_KO_for & CCGAAGCCACAAATTCGAGATG \\
\hline 03404_LB_KO_rev & GTGGGCCATCTAGGCCTCATCTTGACCCCTTGTCGTC \\
\hline 03404_RB_KO_for & CACGGCCTGAGTGGCCTGAGACAAGAGTGGCAGGGT \\
\hline 03404_RB_KO_rev & CGAGCTTAGGAGAGCTGTTGA \\
\hline 03507_LB_KO_for & CGAGTACCTCGAAGGCAAGT \\
\hline 03507_LB_KO_rev & GTGGGCCATCTAGGCCTGACGTCATTCACGATTCCGG \\
\hline 03507_RB_KO_for & CACGGCCTGAGTGGCCGGTGCAAGTGCTTGTAGATGC \\
\hline 03507_RB_KO_rev & TGGCTGTGTTCAAAGGTTCGC \\
\hline 03541_LB_KO_for & CATCTGATGCTCTGAACGTGC \\
\hline 03541_LB_KO_rev & GTGGGCCTGAGTGGCCGTGGTCGATTTGGTTCGACTTATTA \\
\hline 03541_RB_KO_for & CACGGCCATCTAGGCCGGCTGCTGTTGATTGCCAGA \\
\hline 03541_RB_KO_rev & CGTTCAGGTTCTGGGCAGTTT \\
\hline 03665_LB_KO_for & GCTTCGCGCTTATCAGTCTAC \\
\hline 03665_LB_KO_rev & GTGGGCCATCTAGGCCGTTGAACGAGAACGGCTTGC \\
\hline 03665_RB_KO_for & CACGGCCTGAGTGGCCTCATGCTGTTGAGCAGTACAAC \\
\hline 03665_RB_KO_rev & GATCAACGAGAGCGACCACAC \\
\hline 04605_LB_KO_for & CGATGGGTAGGGATGGAATG \\
\hline 04605_LB_KO_rev & GTGGGCCATCTAGGCCCGTGCGCAACGGTGTGTG \\
\hline 04605_RB_KO_for & CACGGCCTGAGTGGCCTGCCAGCCAAGTTTCGCAGAT \\
\hline 04605_RB_KO_rev & CAAGCAACTCGACACGCCATA \\
\hline 04896_LB_KO_for & CGTTCCATCACGAATGCTAACC \\
\hline 04896_LB_KO_rev & GTGGGCCTGAGTGGCCAGTCGAAGCTTGGATAGGCTTTAC \\
\hline 04896_RB_KO_for & CACGGCCATCTAGGCCCTTACCGAGAAACCTGTCGTC \\
\hline 04896_RB_KO_rev & CCTTGGTGTACGAGCATTGGAT \\
\hline 05009_LB_KO_for & CAGCGCGTAGAGAGGTAAAG \\
\hline 05009_LB_KO_rev & GTGGGCCATCTAGGCCTGCAACCGCACAGCCTCC \\
\hline 05009_RB_KO_for & CACGGCCTGAGTGGCCAAGTATGCAGGCTTCTCCCCAT \\
\hline 05009_RB_KO_rev & AGCTGAGTTGGGTTGCGATCAA \\
\hline 05898_LB_KO_for & CCGTGGCTGAGAAAGGCTATC \\
\hline 05898_LB_KO_rev & CACGGCCATCTAGGCCGGCTGGCTAGGGAAAAGAAATTGTA \\
\hline 05898_RB_KO_for & CACGGCCTGAGTGGCCCGCTGACATGATTGCTCCTTGTG \\
\hline 05898_RB_KO_rev & CGGTGCTCCCAGACGAATCAA \\
\hline 06089_LB_KO_for & GAAAGCCCAAGATCGAATCGC \\
\hline
\end{tabular}




\begin{tabular}{|c|c|}
\hline 06089_LB_KO_rev & GTGGGCCATCTAGGCCACAGGCACGACGTTCCATGTT \\
\hline 06089_RB_KO_for & CACGGCCTGAGTGGCCGATGAACGTGACGTGTAGGGA \\
\hline 06089_RB_KO_rev & GCAAGGCTTGTTGTTGTGGTTG \\
\hline 10006_LB_KO_for & CGTACAGAAGAGGCGATCCAA \\
\hline 10006_LB_KO_rev & GTGGGCCTGAGTGGCCGTTGTCCTAGTAGGTCGAGCT \\
\hline 10006_RB_KO_for & CACGGCCATCTAGGCCCCGACGCCCTACGCATC \\
\hline 10006_RB_KO_rev & CATGGTCCCGCTAACTGTG \\
\hline 10686_LB_KO_for & GAGGTGACGTTGATGGATTGG \\
\hline 10686_LB_KO_rev & GTGGGCCTGAGTGGCCTCGCTTGCGTGTTTACGGTC \\
\hline 10686_RB_KO_for & CACGGCCATCTAGGCCTGTGAGAATGTCTCGCTCAGTG \\
\hline 10686_RB_KO_rev & CGAGCGCAACAATGAGACTGAA \\
\hline 10921_LB_KO_for & CTACСТCAACCGACTACTACC \\
\hline 10921_LB_KO_rev & GTGGGCCATCTAGGCCAATTTTTATTGGCCACATTCGTGATT \\
\hline 10921_RB_KO_for & CACGGCCTGAGTGGCCTGTAAACAAGAACTACCAATCTGTC \\
\hline 10921_RB_KO_rev & CAAGCCAGCCAAATGTCACAG \\
\hline 11083_LB_KO_for & TGCTAGAATCAAGTCGCCGATG \\
\hline 11083_LB_KO_rev & GTGGGCCATCTAGGCCGATGACTGTGTGTGTGTGCC \\
\hline 11083_RB_KO_for & CACGGCCTGAGTGGCCCCATAATCGCACGCCTGCAC \\
\hline 11083_RB_KO_rev & GAATCCGAATCGCTCATTGGC \\
\hline 11190_LB_KO_for & CAAGCACAAGGATCTGGGCAAAG \\
\hline 11190_LB_KO_rev & GTGGGCCTGAGTGGCCCTGCCCTGTCGCTCGCG \\
\hline 11190_RB_KO_for & CACGGCCATCTAGGCCGTTCAACTAGATGCGAACGATGC \\
\hline 11190_RB_KO_rev & GCCGAGGTGAAGATCATTGTTG \\
\hline 11513_LB_KO_for & GGGACACGCCAAGTTTGATC \\
\hline 11513_LB_KO_rev & GTGGGCCTGAGTGGCCTGTGCTGGTTGAAGCGGTTG \\
\hline 11513_RB_KO_for & CACGGCCATCTAGGCCGGCGTTGCTCCATTCACGAT \\
\hline 11513_RB_KO_rev & GGAGCGGAAACAAAGAGCAAC \\
\hline 11651_LB_KO_for & TCGGCCTCTCTTGTCAACAATCC \\
\hline 11651_LB_KO_rev & CACGGCCTGAGTGGCCCTTGGCAATGACAGGTTGCGG \\
\hline 11651_RB_KO_for & CACGGCCATCTAGGCCTCGCTTGATCCACCCTTATGCTT \\
\hline 11651_RB_KO_rev & CTCGTTCAGCGTGCTTCGCT \\
\hline 11763_LB_KO_for & CGGAAGAGCGTCATTGAGAAG \\
\hline 11763_LB_KO_rev & GTGGGCCATCTAGGCCCACAGCGCACCTTTCATTTGC \\
\hline 11763_RB_KO_for & CACGGCCTGAGTGGCCATCGAGTCAAGGAACTGTTACAAC \\
\hline 11763_RB_KO_rev & CAGTCGAGCTTCGCTTTCTCA \\
\hline 12149_LB_KO_for & GACCGAACCTGTCAACTTGCTG \\
\hline 12149_LB_KO_rev & CACGGCCTGAGTGGCCGGCGAATGGGATACGTTGAAGC \\
\hline 12149_RB_KO_for & CACGGCCATCTAGGCCGGGAGGCCGCAGTTCGCACGTA \\
\hline 12149_RB_KO_rev & GAACGTCGGCAAAGCAGGC \\
\hline 12178_LB_KO_for & TGACAAGCCAGAAAGCGACAC \\
\hline 12178_LB_KO_rev & GTGGGCCATCTAGGCCTGACCATGTCAAATATCGAGAGATAG \\
\hline 12178_RB_KO_for & CACGGCCTGAGTGGCCAGTTGGACGAGACTTGGCTATG \\
\hline 12178_RB_KO_rev & CAACGAAACGAGAAGGCATCG \\
\hline 12304_LB_KO_for & CGAACACGACAGTACCGACTC \\
\hline 12304_LB_KO_rev & CACGGCCATCTAGGCCTTCCGACAGCGGTTGACATTCAC \\
\hline 12304_RB_KO_for & CACGGCCTGAGTGGCCGGTCGCCGGTGTGACACG \\
\hline 12304_RB_KO_rev & GCTGATTCTGGCCCTTTCATGCC \\
\hline 12318_LB_KO_for & CTTATTGCGTCATCCGCCCAG \\
\hline 12318_LB_KO_rev & GTGGGCCTGAGTGGCCGATCTGTGCAGTGCTGATTGG \\
\hline 12318_RB_KO_for & CACGGCCATCTAGGCCAAGCGTGCCGAGCTGTGG \\
\hline
\end{tabular}




\begin{tabular}{|c|c|}
\hline 12318_RB_KO_rev & CACGCCATGTTGCTGGATGAG \\
\hline 12332_LB_KO_for & САTTCTTАССТСТTTGСТССССТАG \\
\hline 12332_LB_KO_rev & CACGGCCTGAGTGGCCGGGTGGTAAATGCGCTGCTCC \\
\hline 12332_RB_KO_for & CACGGCCATCTAGGCCTCTACCCCCCAAGAATGAGCCAG \\
\hline 12332_RB_KO_rev & CGTTGGCACGGGACATCGTTC \\
\hline 15029_LB_KO_for & GCGCGTCTCAGACCTTGTTG \\
\hline 15029_LB_KO_rev & GTGGGCCTGAGTGGCCGTTGTTTGGTGTTATTCGAGACG \\
\hline 15029_RB_KO_for & CACGGCCATCTAGGCCAGCACACTTTATAATTGCTCTCTC \\
\hline 15029_RB_KO_rev & GCGATTCTGCCAAGCAAGCC \\
\hline 00542_LB_KO_for & CGAGCGGAAGAGCACATCTTG \\
\hline 00542_LB_KO_rev & CACGGCCTGAGTGGCCCTTTGTCAAAACGTTTGAGGATCAGC \\
\hline 00542_RB_KO_for & CACGGCCATCTAGGCССTСTCTCССАССTСАСTGCA \\
\hline 00542_RB_KO_rev & CGAATGGAAGCCCTCTGGGA \\
\hline 05898_LB_KO_for & CCGTGGCTGAGAAAGGCTATC \\
\hline 05898_LB_KO_rev & CACGGCCATCTAGGCCGGCTGGCTAGGGAAAAGAAATTGTA \\
\hline 05898_RB_KO_for & CACGGCCTGAGTGGCCCGCTGACATGATTGCTCCTTGTG \\
\hline 05898_RB_KO_rev & CGGTGCTCCCAGACGAATCAA \\
\hline 10911_LB_KO_for & CTCATCGTTAGCACCGCTCCA \\
\hline 10911_LB_KO_rev & CACGGCCATCTAGGCCATGCGACGCTTCAGGGCATCA \\
\hline 10911_RB_KO_for & CACGGCCTGAGTGGCCGATTGGTGGTGGGCTATGATGC \\
\hline 10911_RB_KO_rev & GTCGTCGTCCGAGTAGCTGTA \\
\hline 11402_LB_KO_for & CAAATCCGATCCGCCTCTTGG \\
\hline 11402_LB_KO_rev & CACGGCCATCTAGGCCGATCAGCCAATCTGAACGAGTCTG \\
\hline 11402_RB_KO_for & CACGGCCTGAGTGGCCGGCTGCTATGCGCTGCTAC \\
\hline 11402_RB_KO_rev & GCAGGTTATCCAACAGAGCGG \\
\hline 05721_LB_KO_for & TCGATCTTCGCTATCCGCCTC \\
\hline 05721_LB_KO_rev & GTGGGCCATCTAGGCCTCTGGAGCGTGTCGTGTATGG \\
\hline 05721_RB_KO_for & CACGGCCTGAGTGGCCGTGTCAAGCAAGAGGCAGTGAC \\
\hline 05721_RB_KO_rev & CCTGTATGCGATATGTGCCACC \\
\hline cib1_lba & AGCTTGGACTAGTAAATGGGACCG \\
\hline cib1_rba & ATTATCCCTTCGCCTTCCCCTTC \\
\hline \multicolumn{2}{|l|}{ Spp1 related } \\
\hline 02729_CBX_for & CACGGATCCATGTCGAGTGATAGAGATCTTTTTATCA \\
\hline 02729_CBX_rev & GTGTCATGAAGTCCTTTTTGGAGGAAATTGCTC \\
\hline 02729_pro1.4_for & TATCATATGGATTGCGACTTGGTATGCGAGGG \\
\hline 02729_pro_rev & ATAGGATCCGATTGCGGAGTTCTGGAACAGAGC \\
\hline 02729_D279A_for & GCTTGGATTGGGCGCCATTGTGATCCCGG \\
\hline 02729_D279A_rev & CCGGGATCACAATGGCGCCCAATCCAAGC \\
\hline SrSpp1_CBX_for & CACGGATCCATGTCCAACGACAGAGACCT \\
\hline SrSpp1_CBX_rev & GTGTCATGAACTCCTTCTTCGCCGTGGAT \\
\hline UhSpp1_CBX_for & CACGGATCCATGTCTGGGGATAGAGATCTG \\
\hline UhSpp1_CBX_rev & GTGTCATGAACTCCTTTTTGGTCGACTTGC \\
\hline HM13_CBX_for & CACCCCGGGATGGACTCGGCCCTCAGC \\
\hline HM13_CBX_rev & GTGCCATGGATTTCTCTTTCTTCTCCAGCCCC \\
\hline sppA_CBX_for & CACGGATCCATGGCTGAGGTCAGTCCAC \\
\hline sppA_CBX_rev & GTGTCATGAACTCGGATGTCGCAGTAGC \\
\hline YPF1_CBX_for & CACGGATCCATGGACAAGTATTTGAATTCATTTGTTG \\
\hline YPF1_CBX_rev & GTGGGATCCAGAAGATTCGTCATCCAAGAGATC \\
\hline 02578_CBX_fwd & CACTCATGAATGACCGACCAAGCTTTGAAAG \\
\hline 02578_CBX_rev & GTGTCATGAAGTAGCCCCCGCCATAACC \\
\hline
\end{tabular}




\begin{tabular}{|c|c|}
\hline 02578_LB_KO_for & ACGGCCTCGTCGCCTTTC \\
\hline 02578_LB_KO_rev & GTGGGCCATCTAGGCCACGACTAACTCTACGCGCATCT \\
\hline 02578_RB_KO_for & CACGGCCTGAGTGGCCGCTCACCTTTCTTCTTGTTCAGTA \\
\hline 02578_RB_KO_rev & CGAGATGTTTCTGACCACAGC \\
\hline $02729 \_\Delta U P R E$ for & GATTTGATTTTGTGGCAAGCCCACGCGC \\
\hline 02729_AUPRE_rev & GCGCGTGGGCTTGCCACAAAATCAAATC \\
\hline 02729__UPRE2_for & TAAAGCCGACTTCGTTGCAAGTCTGTCG \\
\hline 02729__UPRE2_rev & CGACAGACTTGCAACGAAGTCGGCTTTA \\
\hline \multicolumn{2}{|l|}{ qPCR } \\
\hline RT_cib1_spliced_f & GCCTCCCTGCAGCGGATGC \\
\hline RT_cib1_rev & CATCGACGTTGTTTCCGGCCT \\
\hline RT_00904_f & GCTGAAGCAAAGGCCAACTTG \\
\hline RT_00904_r & TTCTTGTCCTGCGCCTGTTTG \\
\hline RT_15034_f & AGGCATGGCTCGACGAGAACA \\
\hline RT_15034_r & GGTAAATCTTGGCGGTGATGGG \\
\hline RT_10287_f & CATCGGAGCGATCAAGGAGATG \\
\hline RT_10287_r & TGGTGACCTTGACCTTGCTGC \\
\hline RT_05352_f & CTGCGGACTATGCGACTCAGTT \\
\hline RT_05352_r & TGTGCTAGCCACTTTTCGCTTT \\
\hline RT_04198_f & AGATCGTCGCGGCCATCTACT \\
\hline RT_04198_r & ATGGCGGACCACACATACACG \\
\hline RT_02729_f & TGGGCTTCACCAAGTCATACACTC \\
\hline RT_02729_r & CGCCTGGAAGAAGTGCATCAC \\
\hline RT_11594_f & TCGTCATATCCTCTTCCGCGT \\
\hline RT_11594_r & ACATCCTCATCCCGTCCAAGA \\
\hline RT_elF2b_f & ATCCCGAACAGCCCAAAC \\
\hline RT_elF2b_r & ATCGTCAACCGCAACCAC \\
\hline mfa1-RT-FW & GCTTTCGATCTTCGCTCAGAC \\
\hline mfa1-RT-RV & CAACAACACAGCTGGAGTAGC \\
\hline RT_pr1_f & ACTACGTGGACCCGCACAAC \\
\hline RT_pr1_r & CGGAGTGGATCAGCTTGCAGTC \\
\hline RT_pr3_f & GAACAACTACAGCAGCCAGGTG \\
\hline RT_pr3_r & GAGACAATAGCTGACATGCGTC \\
\hline RT_pr5_f & TATCGGCCGGAATAGGCTCTG \\
\hline RT_pr5_r & CGCGTACATACAAATGCGTGC \\
\hline RT_pr4_f & CGTTCAAGCCCATCGACA \\
\hline RT_pr4_r & CGTGTGGGATCACATCCATATAAC \\
\hline CC9-qRT-fw & TATGGGTCCTTGACGTTCTC \\
\hline CC9-qRT-rv & GGATCATCCGTAGCCATCTG \\
\hline BBI-qRT-fw & CCGACATCСTCTTCAАCTTCTG \\
\hline BBI-qRT-rv & TTCTCTGAAGCGGCACAC \\
\hline POX12-qRT-fw & CTGAACAAGTTCTTCGCGG \\
\hline POX12-qRT-rv & AGGTCCACGTAGTACTTGTTG \\
\hline ATFP4-qRT-fw & CAGCCTGTGGACATATGC \\
\hline ATFP4-qRT-rv & GCACATGCCCTTAACCTC \\
\hline RT_GAPDH_f & CTTCGGCATTGTTGAGGGTTTG \\
\hline RT_GAPDH_r & TCCTTGGCTGAGGGTCCGTC \\
\hline \multicolumn{2}{|l|}{ Secretion assay } \\
\hline pep1_CBX_for & CACGGATCCATGATGACCACACTGGTGCAAAC \\
\hline pep1_CBX_rev & GTGCCATGGACATGCCAAACATGCTACCGATTC \\
\hline
\end{tabular}




\begin{tabular}{ll}
\hline tin2_CBX_for & CACGGATCCATGAATAGACTTCAGTCCTACACC \\
tin2_CBX_rev & GTGECATGGAAAGAGGGAAGCGAGGGAGC \\
cmu1_CBX_for & CACECCGGGATGAAGTTGAGCGTGTCCATCTTTG \\
cmu1_CBX_rev & GTGECATGGAGGTGCACTTGTTGGCGTGG \\
\hline
\end{tabular}

\subsubsection{Other materials}

Whatman ${ }^{\circledR}$ gel blotting paper (Sigma-Aldrich), rotating wheel, glass beads 150-212 microns (SigmaAldrich), nylon membrane Hybond N+ (Amersham), nitrocellulose membrane (Carl Roth), Petri dishes (Greiner, Carl Roth), $1.5 \mathrm{ml}$ and $2 \mathrm{ml}$ reaction vessels (Eppendorf, Carl Roth), $0.2 \mathrm{ml}$ PCR tubes, $15 \mathrm{ml}$ and $50 \mathrm{ml}$ reaction tubes (Sarstedt), $1.5 \mathrm{ml}$ polystyrene cuvettes (Carl Roth), sterile filter $0.20 \mu \mathrm{m}$ and $0.45 \mu \mathrm{m}$ (Carl Roth), $1 \mathrm{ml}$ disposable syringes (B. Braun), pipette tips $10 \mu \mathrm{l}, 200 \mu \mathrm{l}, 1000 \mu \mathrm{l}$ (Sarstedt), pipette tips for RNA $10 \mu \mathrm{l}, 200 \mu \mathrm{l}, 1000 \mu \mathrm{l}$ (Biozym)

\subsubsection{Buffers and Solutions}

Standard buffers and solutions were prepared according to Ausubel et al. (1987), Sambrook et al. (1989) and Sambrook and Russell (2001). Other buffers and solutions are listed at the respective methods.

\subsubsection{Liquid and solid media}

Table 5.7: E. coli media

\begin{tabular}{ll}
\hline Name & Recipe \\
\hline YT solid medium & $8 \mathrm{~g}$ tryptone \\
(Sambrook et al., 1989) & $5 \mathrm{~g}$ yeast extract \\
& $5 \mathrm{~g} \mathrm{NaCl}$ \\
& $15 \mathrm{~g}$ agar \\
& Fill up with $\mathrm{H}_{2} \mathrm{O}_{\text {bid }}$ to $1 \mathrm{I}$ and autoclave. \\
& Added $100 \mu \mathrm{g} / \mathrm{ml}$ ampicillin (f.c.) for selection of positive \\
& transformants. \\
& $16 \mathrm{~g}$ tryptone \\
& $10 \mathrm{~g}$ yeast extract \\
dYT liquid media & $5 \mathrm{~g} \mathrm{NaCl}$ \\
(Sambrook et al., 1989) & Fill up with $\mathrm{H}_{2} \mathrm{O}_{\text {bid }}$ to $1 \mathrm{I}$ and autoclave. \\
& Added $100 \mu \mathrm{g} / \mathrm{ml}$ ampicillin (f.c.) for selection of positive \\
& transformants.
\end{tabular}


Table 5.8: $U$. maydis media

\begin{tabular}{|c|c|}
\hline Name & Recipe \\
\hline CM solid medium & $1.5 \mathrm{~g} \mathrm{NH}_{4} \mathrm{NO}_{3}$ \\
\hline \multirow[t]{11}{*}{ (Holliday) } & $2.5 \mathrm{~g}$ casamino acids \\
\hline & $0.5 \mathrm{~g}$ Herring Sperm DNA \\
\hline & $1 \mathrm{~g}$ yeast extract \\
\hline & $10 \mathrm{ml}$ vitamin solution (see below) \\
\hline & $62.5 \mathrm{ml}$ salt solution (see below) \\
\hline & $0.5 \mathrm{ml}$ trace element solution (see below) \\
\hline & $20 \mathrm{~g}$ agar \\
\hline & Fill up with $\mathrm{H}_{2} \mathrm{O}_{\text {bid }}$ to $980 \mathrm{ml}$ and adjust $\mathrm{pH}$ with $\mathrm{NaOH}$ to $\mathrm{pH} 7.0$ \\
\hline & and autoclave. After autoclaving add $2 \%(\mathrm{v} / \mathrm{v})$ of a $50 \%$ glucose \\
\hline & solution ( $1 \%$ f.c.) or $2 \%(v / v)$ of a $25 \%$ arabinose solution ( $1 \%$ \\
\hline & f.c.) \\
\hline CM liquid medium & $1.5 \mathrm{~g} \mathrm{NH}_{4} \mathrm{NO}_{3}$ \\
\hline \multirow[t]{10}{*}{ (Holliday) } & $2.5 \mathrm{~g}$ casamino acids \\
\hline & $0.5 \mathrm{~g}$ Herring Sperm DNA \\
\hline & $1 \mathrm{~g}$ yeast extract \\
\hline & $10 \mathrm{ml}$ vitamin solution (see below) \\
\hline & $62.5 \mathrm{ml}$ salt solution (see below) \\
\hline & $0.5 \mathrm{ml}$ trace element solution (see below) \\
\hline & fill up with $\mathrm{H}_{2} \mathrm{O}_{\text {bid }}$ to $980 \mathrm{ml}$ and adjust $\mathrm{pH}$ with $\mathrm{NaOH}$ to $\mathrm{pH} 7.0$ \\
\hline & and autoclave. After autoclaving add $2 \%(\mathrm{v} / \mathrm{v})$ of a $50 \%$ glucose \\
\hline & solution ( $1 \%$ f.c.) or $2 \%(v / v)$ of a $25 \%$ arabinose solution ( $1 \%$ \\
\hline & f.c.) \\
\hline Salt solution & $8 \mathrm{~g} \mathrm{KH}_{2} \mathrm{PO}_{4}$ \\
\hline \multirow[t]{3}{*}{ (Holliday) } & $2 \mathrm{~g} \mathrm{Na}_{2} \mathrm{SO}_{4}$ \\
\hline & $1 \mathrm{~g} \mathrm{MgSO}_{4} * 7 \mathrm{H}_{2} \mathrm{O}$ \\
\hline & Fill up with $\mathrm{H}_{2} \mathrm{O}_{\text {bid }}$ to $1 \mathrm{I}$ and sterile filtrate. \\
\hline Trace elements solution & $60 \mathrm{mg} \mathrm{H}_{3} \mathrm{BO}_{3}$ \\
\hline \multirow[t]{6}{*}{ (Holliday) } & $191 \mathrm{mg} \mathrm{MnCl} 2$ \\
\hline & $400 \mathrm{mg} \mathrm{ZnCl} 2$ \\
\hline & $47 \mathrm{mg} \mathrm{NaMoO}_{4} * 2 \mathrm{H}_{2} \mathrm{O}$ \\
\hline & $140 \mathrm{mg} \mathrm{FeCl}{ }_{3} * 6 \mathrm{H}_{2} \mathrm{O}$ \\
\hline & $557 \mathrm{mg} \mathrm{CuSO}_{4} * 5 \mathrm{H}_{2} \mathrm{O}$ \\
\hline & Fill up with $\mathrm{H}_{2} \mathrm{O}_{\text {bid }}$ to $1 \mathrm{I}$ and sterile filtrate. \\
\hline Vitamin solution & $100 \mathrm{mg}$ thiamin \\
\hline \multirow[t]{3}{*}{ (Holliday) } & $20 \mathrm{mg}$ calcium pantothenate \\
\hline & 50 mg 4-Aminobenzoic acid \\
\hline & 20 mg nicotinic acid \\
\hline
\end{tabular}




\begin{tabular}{|c|c|}
\hline & 20 mg choline chloride \\
\hline & 40 mg myo-inositol \\
\hline & $50 \mathrm{mg}$ folic acid \\
\hline & Fill up with $\mathrm{H}_{2} \mathrm{O}_{\text {bid }}$ to $1 \mathrm{I}$ and sterile filtrate. \\
\hline \multirow{5}{*}{$\begin{array}{l}\text { NSY glycerin } \\
\text { (storage medium for }-80^{\circ} \mathrm{C} \text { ) }\end{array}$} & $8 \mathrm{~g}$ nutrient broth \\
\hline & $1 \mathrm{~g}$ yeast extract \\
\hline & $5 \mathrm{~g}$ sucrose \\
\hline & $800 \mathrm{ml} 87 \%$ glycerin \\
\hline & Fill up with $\mathrm{H}_{2} \mathrm{O}_{\text {bid }}$ to $1 \mathrm{I}$ and autoclave. \\
\hline \multirow{6}{*}{$\begin{array}{l}\text { Regeneration agarlight } \\
\text { (Schulz et al., 1990) }\end{array}$} & $15 \mathrm{~g}(\mathrm{w} / \mathrm{v}) \mathrm{BD} \mathrm{Difco}^{\mathrm{TM}}$ Agar \\
\hline & $182.2 \mathrm{~g} \mathrm{(w/v)} \mathrm{sorbitol}$ \\
\hline & $10 \mathrm{~g}(\mathrm{w} / \mathrm{v})$ yeast extract \\
\hline & $4 \mathrm{~g}(\mathrm{w} / \mathrm{v}) \mathrm{BD}$ Bacto $^{\mathrm{TM}}$ yeast extract \\
\hline & $4 \mathrm{~g}(\mathrm{w} / \mathrm{v})$ sucrose \\
\hline & Fill up with $\mathrm{H}_{2} \mathrm{O}_{\text {bid }}$ to $1 \mathrm{I}$ and autoclave. \\
\hline \multirow{4}{*}{$\begin{array}{l}\text { YEPS light liquid medium } \\
\text { (Tsukuda et al., 1988) }\end{array}$} & 10 g yeast extract \\
\hline & $4 \mathrm{~g}$ peptone \\
\hline & $4 \mathrm{~g}$ sucrose \\
\hline & Fill up with $\mathrm{H}_{2} \mathrm{O}_{\text {bid }}$ to $1 \mathrm{I}$ and autoclave. \\
\hline \multirow[t]{5}{*}{ YNB liquid medium } & $1.7 \mathrm{~g}$ Yeast nitrogen base \\
\hline & $\begin{array}{l}\text { Fill up with } \mathrm{H}_{2} \mathrm{O}_{\text {bid }} \text { to } 1 \mathrm{I} \text {, adjust with } 5 \mathrm{M} \mathrm{NaOH} \text { to } \mathrm{pH} 5.6 \text { and } \\
\text { autoclave. }\end{array}$ \\
\hline & After autoclaving add $2 \%(\mathrm{v} / \mathrm{v})$ of a $50 \%$ glucose solution $(1 \%$ \\
\hline & f.c.) or $2 \%(v / v)$ of a $25 \%$ arabinose solution ( $1 \%$ f.c.) as well as \\
\hline & $0.2 \%(v / v) 20 \%$ ammoinium sulfate. \\
\hline \multirow[t]{5}{*}{ YNB solid medium } & $1.7 \mathrm{~g}$ Yeast nitrogen base \\
\hline & $\begin{array}{l}\text { Fill up with } \mathrm{H}_{2} \mathrm{O}_{\text {bid }} \text { to } 1 \mathrm{I} \text {, adjust with } \mathrm{XXX} \text { to } \mathrm{pH} 5 . \mathrm{X} \text {. and } \\
\text { autoclave. }\end{array}$ \\
\hline & After autoclaving add $2 \%(\mathrm{v} / \mathrm{v})$ of a $50 \%$ glucose solution $(1 \%$ \\
\hline & f.c.) or $2 \%(v / v)$ of a $25 \%$ arabinose solution ( $1 \%$ f.c.) as well as \\
\hline & ammoinium sulfate (20\%) as carbon source (0.2\% f.c.). \\
\hline \multirow[t]{4}{*}{ PD-Charcoal solid medium } & $24 \mathrm{~g}$ potato dextrose broth \\
\hline & $8 \mathrm{~g}$ charcoal \\
\hline & $20 \mathrm{~g}$ agar \\
\hline & Fill up with $\mathrm{H}_{2} \mathrm{O}_{\text {bid }}$ to $1 \mathrm{I}$ and autoclave. \\
\hline
\end{tabular}




\subsubsection{Plasmids}

Table 5.9: In this work used plasmids

\begin{tabular}{|c|c|c|c|}
\hline Name & Resistance & Reference & Description \\
\hline pCR2.1 & $\begin{array}{l}\mathrm{Amp}^{\mathrm{R}} \\
\mathrm{Kan}^{\mathrm{R}}\end{array}$ & Invitrogen & $\begin{array}{l}\text { Cloning of PCR and ligated } \\
\text { products }\end{array}$ \\
\hline p123 & $A m p^{R}$ & Aichinger et al. (2003) & $\begin{array}{l}\text { Consists of an otef promoter and } \\
\text { GFP. For the integration of } \\
\text { constructs into the ip locus. }\end{array}$ \\
\hline p123-mCherry & $A m p^{R}$ & Teichmann et al. (2010) & $\begin{array}{l}\text { Similar to p123. Consists of } \\
\text { mCherry instead of GFP. }\end{array}$ \\
\hline pcib1-3xGFP & $\begin{array}{l}\mathrm{Amp}^{\mathrm{R}} \\
\mathrm{Kan}^{\mathrm{R}}\end{array}$ & Heimel et al. (2010a) & $\begin{array}{l}\text { For C-terminal fusion of } 3 \times G F P \text { to } \\
\text { cib1 in the cib1 locus. }\end{array}$ \\
\hline pBS-hhn & $A m p^{R}$ & Kämper (2004) & $\begin{array}{l}\text { Consists of the hygromycin } \\
\text { resistance cassette. }\end{array}$ \\
\hline pUMa389 & $A m p^{R}$ & Becht et al. (2006) & $\begin{array}{l}\text { For C-terminal fusion with } \\
\text { enhanced GFP as reporter gene. } \\
\text { Consists nourseothricin resistance } \\
\text { cassette }\end{array}$ \\
\hline pUMa793 & $A m p^{R}$ & Becht et al. (2006) & $\begin{array}{l}\text { For C-terminal } 3 \times \mathrm{HA} \text { fusion. } \\
\text { Consists nourseothricin resistance } \\
\text { cassette. }\end{array}$ \\
\hline pUMa707 & $A m p^{R}$ & Zarnack et al. (2006) & $\begin{array}{l}\text { Promoter replacement with } \\
\text { regulated tetracycline promoter. } \\
\text { Consists of a phleomycin } \\
\text { resistance cassette. }\end{array}$ \\
\hline
\end{tabular}

\section{In this work generated plasmids}

Cloning steps of plasmids were checked by restriction analysis and introduced PCR amplicons were verified by sequencing.

\section{Gene deletions}

All gene deletions were performed according to Kämper (2004). Flanking gene borders ( $\sim 1 \mathrm{~kb})$ were PCR amplified from genomic DNA of U. maydis (UM521), which integrated a SfiI restriction site on each border. Both fragments were ligated to a SfiI $\mathrm{Hyg}^{\mathrm{R}}$ fragment of the plasmid pBS-hhn (Kämper, 2004). The ligation product integrated into the pCR2.1 TOPO vector (Invitrogen).

\section{pCR2.1-cib1-GFP and pCR2.1-cib1-3xHA}

For the cib1-GFP fusion, the $5.5 \mathrm{~kb}$ SfiI 3xGFP-Hyg ${ }^{\mathrm{R}}$ fragment of plasmid pcib1-3xGFP (Heimel et al., 2010a) was replaced with the $2.5 \mathrm{~kb}$ SfiI GFP-Nat ${ }^{\mathrm{R}}$ fragment from pUMa389 (Becht et al., 2006) to generate the plasmid pCR2.1-cib1-GFP. The resulting vector was used to generate plasmid pCR2.1cib1-3xHA by exchanging the SfiI GFP-Nat ${ }^{\mathrm{R}}$ cassette with a $1.8 \mathrm{~kb}$ SfiI $3 \mathrm{xHA}^{-\mathrm{Nat}^{\mathrm{R}}}$ fragment from pUMa793 (Becht et al., 2006).

\section{pCR2.1-cib1 ${ }^{T 381 A}-$ GFP and PCR2.1-cib1 $1^{T 381 E_{-}}$GFP}

To generate the phospho-null ( $c i b 1^{\mathrm{T} 381 \mathrm{~A}}-$ GFP) or phospho-mimic ( $c i b 1^{\mathrm{T} 381 E}-G F P$ ) mutation in cib1GFP, a point mutation was introduced into the ORF of cib1 by standard PCR procedures. Cloning of 
the PCR fragment followed the procedure as described for pCR2.1-cib1-GFP, yielding the plasmids pCR2.1-cib1 $1^{\text {T381A }}$-GFP and pCR2.1-cib1 $1^{\text {T381E }}-$ GFP.

PCR2.1-cib1 $1_{-G 68 A}^{\text {S46A and PCR2.1-cib1 }}{ }^{\text {S468E }}$-GFP

To generate the phospho-null ( $c i b^{\text {S468A }}$ GFP) or phospho-mimic ( $c i b 1^{S 468 E}-G F P$ ) mutation in cib1-GFP, a point mutation was introduced into the ORF of cib1 by standard PCR procedures. Cloning of the PCR fragment followed the procedure as described for pCR2.1-cib1-GFP, yielding the plasmids pCR2.1$c i b 1^{\text {S468A }}$-GFP and pCR2.1-cib1 ${ }^{\text {S468E }}$-GFP.

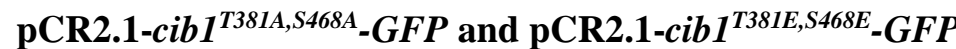

To generate the double phospho-null ( $c i b 1^{\text {T381A,S468A }}-G F P$ ) or double phospho-mimic ( $c i b 1^{T 381 E, S 468 E_{-}}$ GFP) mutations in cib1-GFP, point mutations were introduced into the ORF of cib1 by fusion PCR. Cloning of the PCR fragments followed the procedure as described for PCR2.1-cib1-GFP, yielding the plasmids pCR2.1-cib1 $1^{\text {T381A,S468A }}$-GFP and pCR2.1-cib1 $1^{\text {T381E,S468E }}$-GFP.

\section{pCR2.1-P tef:tTA-tetO:cib1-GFP}

For replacement of the cib1 promoter with a tetracycline-regulated promoter, $1 \mathrm{~kb}$ upstream of the cib1 start codon and $1 \mathrm{~kb}$ of the cib1 open reading frame (ORF) were PCR amplified from genomic DNA, ligated to the SfiI cassette of pUMa707 (Zarnack et al., 2006) and integrated in the pCR2.1 TOPO vector (Invitrogen) generating plasmid pCR2.1-P tef:tTA-tetO:cib1-GFP

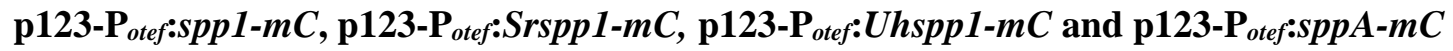

To generate the spp1-mCherry fusion, the ORF of spp1 (UMAG_02729, UM521) lacking the stop codon was PCR amplified from genomic DNA introducing a BamHI site at the 5' end and a BspHI site at the 3' end and integrated into p123-mCherry (Teichmann et al., 2010), to yield p123-Potef:spp1-mC. Cloning of orthologous genes from Sporisorium reilianum Srspp1 (sr13785, strain SRZ1), Ustilago hordei Uhspp1 (UHOR_04354, strain Uh4857-4) and Aspergillus nidulans sppA (ANID_08681, strain

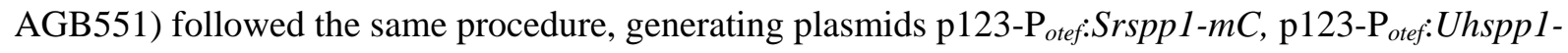
$m C$ and $\mathrm{p} 123-\mathrm{P}_{\text {otef:SppA- }} m C$, respectively.

\section{p123-Potef:YPF1-mC}

For cloning of S. cerevisiae YPF1 the ORF (YKL100C, strain sigma 1287) was PCR amplified from genomic DNA introducing BamHI sites at the 5' and 3' end removing the stop codon and integrated into p123-mCherry (Teichmann et al., 2010) to yield plasmid p123-P otef:YPF1-mC. 


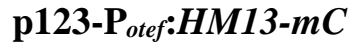

The cDNA of the human HM13 (BC062595, cDNA clone) was PCR amplified from the vector pCS6(BC062595)-TCH1303-GVO-TRI (BioCat) introducing an XmaI site at the 5' end, a NcoI site at the 3' end and removing the stop codon and subsequently ligated into p123-mCherry (Teichmann et al., 2010) to yield p123-Potef:HM13-mC.

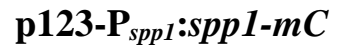

To replace the otef promoter in $\mathrm{p} 123-\mathrm{P}_{\text {otef:Spp1-mC }}$ with the spp1 promoter, a $1.4 \mathrm{~kb}$ spp1 promoter fragment was PCR amplified introducing a NdeI site at the 5' end and a BamHI site at the 3' end. The PCR fragment was integrated into p123-P otef:spp1-mC to generate $123-\mathrm{P}_{\text {spp1 }}$ :spp1-mC.

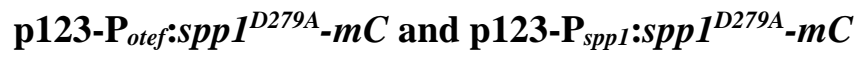

To generate the catalytically inactive version of $\operatorname{spp} 1$ ( $\operatorname{spp} 1^{D 279 A}$ ), a point mutation was introduced into the ORF of spp1 by standard PCR procedures. Cloning of the PCR fragment followed the procedure as described for $\mathrm{p} 123-\mathrm{P}_{\text {otef:spp1- }} \mathrm{mC}$ and $\mathrm{p} 123-\mathrm{P}_{\text {spp } 1}:$ spp1- $m C$, yielding the plasmids $\mathrm{p} 123-\mathrm{P}_{\text {otef:spp }} 1^{\text {D279A }}$ $m C$ and $\mathrm{p} 123-\mathrm{P}_{\text {spp } 1}:$ spp $1^{\mathrm{D279A}}-m C$, respectively.

\section{p123-P ote $_{\text {:Spp1-GFP and p123-P }}$ otef:spp1 $1^{\text {D279A }}-$ GFP}

To generate the spp1-GFP fusion as well as a variant with the catalytically inactive $\operatorname{spp} 1$ (spp $1^{\text {D279A }}$ GFP), similar cloning procedures were used as described for $\mathrm{p} 123-\mathrm{P}_{\text {otef:spp1-mC }}$ and $\mathrm{p} 123-$ $\mathrm{P}_{\text {otef:Spp1 }}{ }^{\text {D279A }}-m C$, respectively. PCR fragments were integrated into p123 (Aichinger et al., 2003) instead of p123-mCherry, generating the plasmids p123-P otef:spp1-GFP and p123-P otef:Spp $1^{\text {D279A }}-G F P$.

\section{p123-P otef:pep1-mC}

To generate the pep1-mCherry fusion, the ORF of pep1 (UMAG_01987, UM521) lacking the stop codon was PCR amplified from genomic DNA introducing a BamHI site at the 5' end and a NcoI site at the 3' end and integrated into p123-mCherry (Teichmann et al., 2010), to yield p123-P $\mathrm{P}_{\text {otef:pep1-mC. }}$

\section{p123-Pote:tin2-mC}

To generate the tin2-mCherry fusion, the ORF of tin2 (UMAG_05302, UM521) lacking the stop codon was PCR amplified from genomic DNA introducing a BamHI site at the 5' end and a NcoI site at the 3' end and integrated into p123-mCherry (Teichmann et al., 2010), to yield p123-Potef:tin2-mC.

\section{p123-P otef:cmu1-mC}

To generate the cmu1-mCherry fusion, the ORF of cmu1 (UMAG_05731, UM521) lacking the stop codon was PCR amplified from genomic DNA introducing an XmaI site at the 5' end and a NcoI site at the 3' end and integrated into p123-mCherry (Teichmann et al., 2010), to yield p123-P otef:Cmu1-mC. 


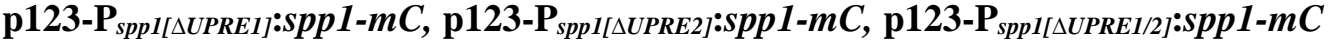

To generate the deletion of UPRE1 (Chapter 3.3.10) in the promoter of spp1, the promoter fragments

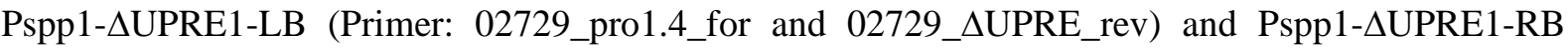
(Primer: 02729_AUPRE_for and 02729_pro_rev) were PCR amplified. To generate a full-length promoter lacking the UPRE1, an overlapping PCR (Primer: 02729_pro1.4_for and 02729_pro_rev) was performed on both sub-fragments. The generated Pspp1- $\triangle$ UPRE1 full-length fragment was cloned as

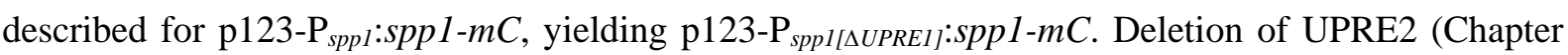
3.3.10) in the spp1 promoter was performed similar as described for deletion of UPRE1, by using different primers for generation of the promoter sub-fragments (02729_pro1.4_for and 02729_AUPRE2_rev or 02729_AUPRE2_for and 02729_pro_rev), generating the plasmid p123$\mathrm{P}_{\text {SPP1[AUPRE2] }}$ :spp1-mC. To generate a double deletion of UPRE1 and UPRE2, the promoter sub-fragments

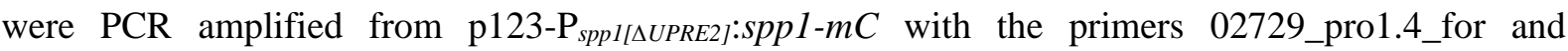
02729_AUPRE_rev (Pspp1-_UPRE1/2-LB) or 02729_AUPRE_for and 02729_pro_rev (Pspp1$\triangle \mathrm{UPRE} 1 / 2-\mathrm{RB})$ and cloned as described for p123-P $\mathrm{P}_{\text {sp1 }[\triangle U P R E 1]}$ :spp1-mC, yielding p123$\mathrm{P}_{\text {spp1[AUPRE1/2] }}:$ spp1-mC.

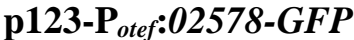

To generate the UMAG_02578-GFP fusion, the ORF of UMAG_02578 lacking the stop codon was PCR amplified from genomic DNA (UM521) introducing a BspHI site at the 5' and the 3' end, and integrated

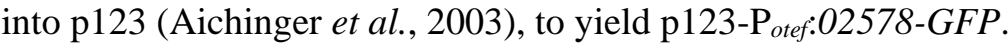

\subsection{Standard methods of microbiology}

\subsubsection{Cultivation of $E$. coli}

E. coli strains were cultivated either as shake cultures at $200 \mathrm{rpm}$ or on solid media under aerobic conditions at $37^{\circ} \mathrm{C}$. For inoculation of E. coli, dYT liquid medium and YT solid medium were used (Ausubel et al., 1987; Sambrook et al., 1989). For plasmid selection, the antibiotics ampicillin (100 $\mu \mathrm{g} / \mathrm{ml}$ ) were used. Cultures were stored at $-80^{\circ} \mathrm{C}$ in glycerol, and were streaked out and grown on YT solid media before further inoculation.

\subsubsection{Transformation of $\boldsymbol{E}$. coli}

To preparation of chemical-competent bacterial cells, a modified protocol according to Hanahan et al. (1991) was used. $100 \mathrm{ml}$ of SOB medium were inoculated with $1 \mathrm{ml}$ of a fresh TOP10 overnight culture and incubated to an $\mathrm{OD}_{600}$ of 0.5 at $37^{\circ} \mathrm{C}$ and $200 \mathrm{rpm}$. The cells were centrifuged (4.000 rpm, $10 \mathrm{~min}$, $4^{\circ} \mathrm{C}$ ), resuspended in one-third of the starting volume in ice-cold CCMB80 buffer and incubated on ice 
for $20 \mathrm{~min}$. The cells were centrifuged again ( $4.000 \mathrm{rpm}, 10 \mathrm{~min}, 4^{\circ} \mathrm{C}$ ) and resuspended in $1 / 12$ of the starting volume. The cell suspension was aliquoted into $50 \mu \mathrm{l}$ portions and stored at $-80^{\circ} \mathrm{C}$.

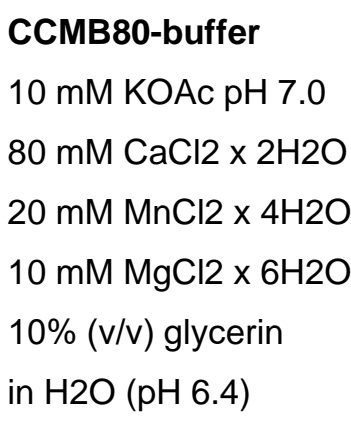

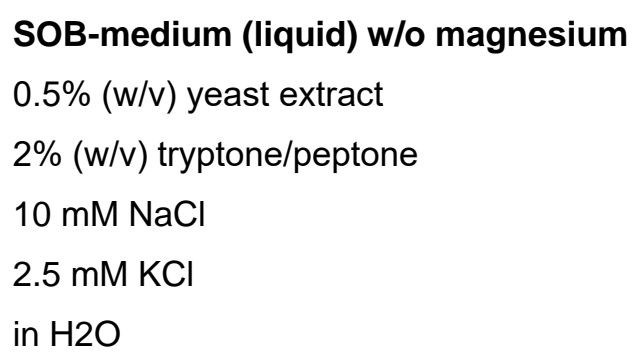

For the transformation of $E$. coli strains, the cells were thawed on ice, and $10 \mu$ l plasmid solution (1-5 ng plasmid) or the ligation mixture was added to a volume of $50 \mu$ l cells and incubated on ice for 15 min. After a heat shock of $30-60 \mathrm{sec}$ at $42^{\circ} \mathrm{C}$, the transformation mixture was streaked out on YT plates with the appropriate antibiotic and incubated overnight at $37^{\circ} \mathrm{C}$.

\subsubsection{Cultivation of $U$. maydis}

U. maydis strains were cultured in YEPS light, $\mathrm{CM}$ or YNB liquid medium at $28^{\circ} \mathrm{C}$ and $180 \mathrm{rpm}$ shaking in baffled flasks. For cultivation on solid media, CM, YNB or PD solid medium was used. The following antibiotics were used for selection: Carboxin ( $2 \mu \mathrm{g} / \mathrm{ml}$, Sigma-Aldrich, 45371), Hygromycin B (200 $\mu \mathrm{g} / \mathrm{ml}$, InvivoGen, ant-hg-5), G418/geneticin (400 $\mu \mathrm{g} / \mathrm{ml}$, Merck/Calbiochem, 345810), Phleomycin (40 $\mu \mathrm{g} / \mathrm{ml}$, InvivoGen, ant-ph-1) or Nourseothricin (50 $\mu \mathrm{g} / \mathrm{ml}$, Werner BioAgents, clonNAT). For longterm storage of the strains, strains were inoculated in YEPS $S_{\text {light }}$ and grown overnight at $28^{\circ} \mathrm{C}$. Subsequently, $0.9 \mathrm{ml}$ of the culture was mixed with $0.9 \mathrm{ml}$ NSY-glycerol and stored at $-80^{\circ} \mathrm{C}$. For further use of the stored strains, cells were streaked out on CM solid media and grown overnight at $28^{\circ} \mathrm{C}$. For RNA isolation of strains as well as stress assays performed on solid media (ER stress, cell wall stress and growth in hypoxia), strains were inoculated in liquid or solid YNB medium, respectively.

\subsubsection{Measurement of cell density in $U$. maydis}

The cell density of liquid cultures was determined photometrically using a UV-1202 (Shimadzu) at 600 $\mathrm{nm}$. In order to ensure a linear reference, the cell suspensions were diluted to a value below $\mathrm{OD}_{600}$ of 1.0. The respective culture medium was used as a reference. An $\mathrm{OD}_{600}$ of 1.0 corresponds to $1-5 \times 10^{7}$ cells/ml. 


\subsubsection{Transformation of $\boldsymbol{U}$. maydis}

\section{Generation of $U$. maydis protoplasts}

The transformation protocol was modified according to Schulz et al. (1990) and Gillissen et al. (1992). YEPS $_{\text {light }}$ liquid medium was inoculated with strains grown on solid medium and shaken in baffled flasks at $28^{\circ} \mathrm{C}$ overnight. Thus, the preculture was diluted in $50 \mathrm{ml}$ of YEPS light $_{\text {to }}$ an $\mathrm{OD}_{600}$ of 0.2 and shaken at $28^{\circ} \mathrm{C}$ to a cell density of $\mathrm{OD}_{600}$ of $0.8-1.0$. Subsequently, cells were centrifuged (3500 rpm, $5 \mathrm{~min}$, $4^{\circ} \mathrm{C}$, Heraeus Biofuge Stratos, rotor \#3057) and washed once with ice-cold $25 \mathrm{ml} \mathrm{SCS}$. For protoplastation of the cells, $2 \mathrm{ml}$ SCS with $20 \mathrm{mg} / \mathrm{ml}$ lysing enzyme (Sigma-Aldrich, L1412) was added. The protoplastation of the cells was monitored at room temperature microscopically until two-thirds of the cigar-shaped cells start to form spherical shapes on their cell poles. After protoplastation (5-15 min), $10 \mathrm{ml}$ of ice-cold SCS was added and the protoplasts were gently pelleted by centrifugation at $2000 \mathrm{rpm}$ (10 min, $4^{\circ} \mathrm{C}$, Heraeus Biofuge Stratos, rotor \#3057). The latter wash step was repeated once, to completely remove the lysing enzyme of the cells and stop the protoplastation. Cells were then washed with $10 \mathrm{ml}$ of ice-cold STC, and the pellet was resuspended in $0.5 \mathrm{ml}$ of ice-cold STC and aliquoted into $100 \mu \mathrm{l}$ portions. Protoplasts can be stored for $3-4 \mathrm{~h}$ on ice or at $-80^{\circ} \mathrm{C}$ for several months.

\section{Transformation of $U$. maydis protoplasts}

For transformation, $100 \mu \mathrm{l}$ of protoplasts were incubated with 1 to $10 \mu \mathrm{l}$ of linearized plasmids or PCR amplified and purified DNA constructs (3-5 $\mu \mathrm{g})$ and $1 \mu \mathrm{l}$ of heparin solution $(15 \mathrm{mg} / \mathrm{ml})$ for $15 \mathrm{~min}$ on ice. Thereafter, $500 \mu \mathrm{l}$ STC/PEG solution was added and further incubated for $15 \mathrm{~min}$ on ice. Subsequently, the entire transformation approach was gently streaked out on a regeneration agar medium supplemented with antibiotic for selection of positive transformants. After 4 to 7 days of incubation at $28^{\circ} \mathrm{C}$, the grown colonies were single-streaked with toothpicks on antibiotic-containing CM plates.

SCS

Solution 1:

$20 \mathrm{mM}$ sodium citrate

$1 \mathrm{M}$ sorbitol

in $\mathrm{H} 2 \mathrm{O}$

Solution 2:

$20 \mathrm{mM}$ citric acid

$1 \mathrm{M}$ sorbitol

in $\mathrm{H} 2 \mathrm{O}$
STC

$10 \mathrm{mM}$ Tris- $\mathrm{Cl}, \mathrm{pH} 7.5$

$100 \mathrm{mM} \mathrm{CaCl}_{2}$

$1 \mathrm{M}$ Sorbitol

in $\mathrm{H} 2 \mathrm{O}$

The solution can be autoclaved for sterilization.

\section{STC/PEG}

$90 \mathrm{ml} \mathrm{STC}$

60 g PEG 4000

Dissolve PEG in STC buffer. The buffer

can be autoclaved for sterilization. 
Add solution 2 to solution 1 until a pH of 5.8 is reached. The final buffer can be autoclaved for sterilization.

\subsubsection{Microscopic analysis of $U$. maydis strains}

\section{Fluorescent microscopic analysis of $U$. maydis strains}

For fluorescence microscopic analysis, $100 \mu \mathrm{l}$ of $2 \%(\mathrm{w} / \mathrm{v})$ agarose was applied on a glass slide and immediately a second glass slide was put on top, to generate a flat surface. After $5 \mathrm{~min}$, the second glass slide was removed and $5 \mu \mathrm{l}$ of $U$. maydis culture, with an $\mathrm{OD}_{600}$ of 0.8 , was spotted on the prepared agarose surface and covered with a coverslip. Microscopic analysis was performed using an Axio Imager.M1 (ZEISS) equipped with a CoolSNAP HQ2 CCD camera (PHOTOMETRICS). For fluorescence microscopy, filters with the following excitation and emission spectra were used: GFP 450$490 \mathrm{~nm}$ and 515-565 nm, RFP 550-580 nm and 590-650 nm. Images were processed with ZEN 2.3 blue edition (ZEISS) and ImageJ 1.48 (https://imagej.nih.gov/ij)

\section{Analysis of in planta growth of $U$. maydis}

Chlorazole Black E staining was performed according to Brachmann et al. (2001). For microscopic analysis of cells after TM treatment $U$. maydis strains were grown in $\mathrm{CM}$ to an $\mathrm{OD}_{600}$ of 0.35 . TM was added to a final concentration of $5 \mu \mathrm{g} / \mathrm{ml}$ and cells were incubated for the indicated time to induce the UPR. Microscopic analysis was performed using an Axio Imager.M2 equipped with an AxioCam MRm camera (ZEISS) or an Axio Imager.M1 (ZEISS) equipped with a CoolSNAP HQ2 CCD camera (PHOTOMETRICS). Images were processed with ZEN 2.3 blue edition (ZEISS) and ImageJ 1.48 (https://imagej.nih.gov/ij).

\section{Analysis of ROS formation in planta}

For the detection of reactive oxygen species (ROS) in infected leaf tissue, 3,3'-diaminobenzidine (DAB) was used as described previously in Molina and Kahmann (2007). Briefly, leaves (third leaf) were detached with a razor blade $1 \mathrm{~cm}$ above and $2 \mathrm{~cm}$ below the injection site $24 \mathrm{~h}$ post infection and incubated for $12 \mathrm{~h}$ in $1 \mathrm{mg} / \mathrm{ml} \mathrm{DAB}$ solution under darkness at room temperature. For decolorization, leaves were immersed in ethanol (96 \%) for 48h. For storage of the specimens, the leaves were transferred into $10 \%$ (v/v) glycerol. Brown polymerization products resulting from the reaction of DAB with ROS were microscopically identified using a binocular microscope (Keyence Digital Microscope VHX-500F). Images were processed with ImageJ 1.48 (https://imagej.nih.gov/ij). 


\subsubsection{Induction of ER-Stress in U. maydis}

For induction of ER stress in expression studies, YNB liquid medium was inoculated with $U$. maydis strains and grown over the day. In the evening, the cell density of the culture was measured and adjusted to an $\mathrm{OD}_{600}$ of 0.3 to the next day and grown overnight. Cells were treated with $5 \mu \mathrm{g} / \mathrm{ml}$ tunicamycin (f.c.) and harvested after $4 \mathrm{~h}$ of treatment by centrifugation $\left(4^{\circ} \mathrm{C}, 3500 \mathrm{rpm}, 5 \mathrm{~min}\right.$, Heraeus Biofuge Stratos, rotor \#3047). After centrifugation, cell pellets were flash-frozen in liquid nitrogen. Frozen pellets were stored in $-80^{\circ} \mathrm{C}$ for further use.

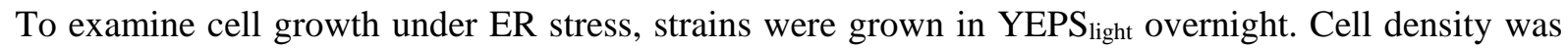
measured and adjusted that it reached an $\mathrm{OD}_{600}$ of 1 after $5 \mathrm{~h}$ of growth. Cells were centrifuged (3500 rpm, 5 min, Heraeus Pico 17) and a 10-fold dilution series starting at an $\mathrm{OD}_{600}$ of 1 was prepared in YNB (without glucose and ammonium sulfate). $3.5 \mu$ of each dilution was spotted on YNB solid medium (with or without a tunicamycin) and incubated for two days at $28^{\circ} \mathrm{C}$.

\subsubsection{Infection of Zea mays with $U$. maydis}

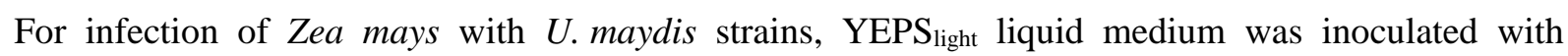
$U$. maydis strains and grown to an $\mathrm{OD}_{600}$ of 0.8 . Cells were harvested by centrifugation (3000 rpm, 5 min, RT, Heraeus Biofuge Stratos) and subsequently resuspended in water (to an $\mathrm{OD}_{600}$ of 1.0). $500 \mu \mathrm{l}$ of the cell suspension was injected with a disposable syringe around $1 \mathrm{~cm}$ above the ground into the interior of the leaf vortex of 7-day-old maize plants of the maize variety "Early Golden Bantam”. The infected plants were grown for eight days in a climatic chamber (CLF Plant Climatics GroBank's TS110). Infection symptoms were rated according to criteria modified from Kämper et al. (2006).

\section{Program GroBanks:}

during the day $8: 00-22: 00,28^{\circ} \mathrm{C}$, light intensity $250 \mu \mathrm{mol}$

at night 22:00 - 8:00, $22^{\circ} \mathrm{C}$

\subsection{Standard methods of molecular biology}

\subsubsection{Handling of nucleic acids}

\section{Determination of DNA concentration}

The concentration of nucleic acids was determined photometrically. An absorbance value of 1 at a wavelength of $260 \mathrm{~nm}$ and a slice thickness of $1 \mathrm{~cm}$, corresponds to a concentration of about $50 \mu \mathrm{g} / \mathrm{ml}$ double-stranded DNA and $33 \mu \mathrm{g} / \mathrm{ml}$ single-stranded DNA. To measure the purity of the DNA, the quotient of the optical densities at the wavelengths $260 \mathrm{~nm}$ and $280 \mathrm{~nm}$ was used (1.8 for pure DNA). 
Lower values indicate contamination with proteins, higher values indicate impurities with salts or sugars. All measurements were performed with a Nanodrop ND-1000 spectrophotometer.

\subsubsection{Isolation of nucleic acids}

\section{Minipreparation of plasmid DNA of $E$. coli}

Plasmid DNA isolation of E. coli was performed according to Sambrook et al. (1989). $1.5 \mathrm{ml}$ of an E. coli overnight culture was pelleted (13000 rpm, $30 \mathrm{sec}$, RT, Heraeus Pico 17). The cell pellet was resuspended in $300 \mu 1$ STET and $30 \mu 1$ lysozyme solution (10 min, 1500 rpm, Vibrax VRX (IKA)) and incubated for $1 \mathrm{~min}$ at $95^{\circ} \mathrm{C}$ in a heating block (Eppendorf Thermomixer compact). The lysed cells and the denatured genomic DNA were centrifuged (13,000 rpm, 15 min, RT, Heraeus Pico 17). The pellet was removed from the aqueous solution using a sterile toothpick. Plasmid DNA was precipitated with 30 $\mu \mathrm{l}$ of Minilysate(III) solution and $500 \mu \mathrm{l}$ of isopropanol, and subsequent centrifugation (13,000 rpm, 15 min, RT, Heraeus Pico 17). The plasmid DNA pellet was washed with $70 \%$ ethanol. After drying, the plasmid DNA pellet was taken up in $100 \mu \mathrm{l}$ of TE buffer containing $20 \mu \mathrm{g} / \mathrm{ml}$ RNase A and dissolved for $20 \mathrm{~min}$ at $50^{\circ} \mathrm{C}$ in a heating block (Eppendorf Thermomixer compact).

\author{
STET buffer \\ $10 \mathrm{mM}$ Tris- $\mathrm{HCl}(\mathrm{pH} 8.0)$ \\ $100 \mathrm{mM} \mathrm{NaCl}$ \\ $1 \mathrm{mM} \mathrm{Na}$-EDTA \\ $5 \%(v / v)$ TritonX-100 \\ in $\mathrm{H}_{2} \mathrm{O}_{\text {bid }}$
}

\begin{tabular}{|l} 
Lysozyme solution \\
$10 \mathrm{mg} / \mathrm{ml}$ lysozyme \\
in $1 \mathrm{x}$ TE buffer
\end{tabular}

\author{
Mini lysate(III) solution \\ $3 \mathrm{M} \mathrm{NaAc}(\mathrm{pH} 4.8)$ \\ in $\mathrm{H}_{2} \mathrm{O}_{\text {bid }}$ \\ in $\mathrm{H}_{2} \mathrm{O}_{\text {bid }}$
}

\section{Genomic DNA isolation of $U$. maydis}

For genomic DNA isolation of U. maydis, $2 \mathrm{ml}$ of a $U$. maydis overnight culture, grown in $3-5 \mathrm{ml}$ of YEPS $_{\text {light }}$ medium, was centrifuged (2 min, $13000 \mathrm{rpm}$, RT, Heraeus Pico 17). The supernatant was discarded and $500 \mu \mathrm{l}$. maydis lysis buffer, as well as $300 \mathrm{mg}$ glass beads, were added to the cell pellet. To disrupt the cells, the mixture was shaken vigorously (10 minutes, $1500 \mathrm{rpm}$, RT, Vibrax VRX (IKA)) and incubated for $15 \mathrm{~min}$ at $65^{\circ} \mathrm{C}$ (Eppendorf Thermomixer compact). After incubation for 5 min on ice, $100 \mu \mathrm{l}$ 8M potassium acetate was added, mixed vigorously and centrifuged (15 min, $13000 \mathrm{rpm}$, RT, Heraeus Pico 17). $500 \mu \mathrm{l}$ of supernatant was transferred into a fresh reaction tube, $300 \mu \mathrm{l}$ isopropanol was added to the supernatant and mixed vigorously before centrifugation (15 min, 13000 rpm, RT, Heraeus Pico 17). After centrifugation, the supernatant was removed and the pellet was washed with 70\% EtOH (5 min, 13000 rpm, RT, Heraeus Pico 17). The supernatant was removed completely and the pellet was dried for about 5 minutes at room temperature and taken up in $35 \mu \mathrm{l}$ of TE buffer with $50 \mu \mathrm{g} / \mathrm{ml} \mathrm{RNaseA}$. Subsequently, the DNA was dissolved at $50^{\circ} \mathrm{C}$ and $850 \mathrm{rpm}$ for $20 \mathrm{~min}$ (Eppendorf 
Thermomixer compact). For quality control, $1 \mu \mathrm{l}$ of the genomic DNA was applied to a $0.8 \%$ agarose gel and analyzed (modified after Hoffman and Winston (1987)).

\section{U. maydis lysis buffer}

$50 \mathrm{mM}$ Tris- $\mathrm{HCl}(\mathrm{pH} 7.5)$

$50 \mathrm{mM} \mathrm{Na} 2$-EDTA

$1 \%(w / v)$ SDS

in $\mathrm{H}_{2} \mathrm{O}_{\text {bid }}$

For usage mix 50:50 with 1x TE.
$8 \mathrm{M}$ potassium acetate (KOAc) 8M KOAC

in $\mathrm{H}_{2} \mathrm{O}_{\text {bid }}$

\section{RNA isolation of $U$. maydis}

This method is based on the protocol of the manufacturer Invitrogen and was used for the preparation of total RNA from U. maydis liquid cultures or infected maize leaves.

A) From U. maydis liquid culture: $15 \mathrm{ml}$ of the cell culture was pelleted (5 min, $3500 \mathrm{rpm}, \mathrm{RT}$, Heraeus Biofuge Stratos, rotor \#3047), the supernatant was discarded and the cell pellet was flash frozen in liquid nitrogen (and optionally stored at $-80^{\circ} \mathrm{C}$ ).

B) From infected maize leaves: A piece of the 3rd leaf (about $1 \mathrm{~cm}$ below the injection site and $2 \mathrm{~cm}$ long) was cut off and immediately flash frozen in liquid nitrogen (and optionally stored at $-80^{\circ} \mathrm{C}$ ). The leaf material was ground in a mortar with liquid nitrogen to a fine powder (possibly stored at $\left.-80^{\circ} \mathrm{C}\right)$.

$1 \mathrm{ml}$ of TRIzol (Invitrogen) was pipetted onto the frozen cell pellet or the plant material and transferred into $2 \mathrm{ml}$ reaction vessels with $300 \mathrm{mg}$ of glass beads. The mixture was shaken (8 min, $2000 \mathrm{rpm}$, Vibrax VRX (IKA)) and incubated for $5 \mathrm{~min}$ at RT. After addition of $200 \mu \mathrm{l}$ chloroform, the samples were mixed briefly (SI Vortex-Genie 2) and incubated for 2 to 3 minutes at RT. After centrifugation (10 min, $13000 \mathrm{rpm}$, Sigma 1-15) the clear supernatant was removed and transferred into a $1.5 \mathrm{ml}$ reaction vessel with $500 \mu \mathrm{l}$ of isopropanol, incubated for $10 \mathrm{~min}$ at RT and centrifuged again (15 min, $13000 \mathrm{rpm}$, Sigma 1-15). The supernatant was removed and the RNA pellet was washed with $750 \mu$ l ethanol (70\%) (13000 rpm, 5 min, Sigma 1-15). The supernatant was removed completely and the RNA pellet was dissolved in $60 \mu \mathrm{l} \mathrm{H} 2 \mathrm{O}$ (RNase free, Ambion) at $55^{\circ} \mathrm{C}$ for $10 \mathrm{~min}$. For quality control, $1 \mu \mathrm{l}$ of the RNA was loaded on an $0.8 \%$ agarose gel. 


\subsection{3 in vitro modification of DNA}

Protocols modified after Sambrook et al. (1989)

\section{Restriction digest of DNA}

For cleavage of double-stranded DNA, type II restriction endonucleases were used with the buffer conditions recommended by the manufacturer. A typical reaction mixture contained:

$$
\begin{aligned}
& 0.5 \mu \mathrm{g} \text { DNA } \\
& 10 \mu \mathrm{g} \text { albumin (if recommended) } \\
& 0.5 \mathrm{U} \text { restriction enzyme } \\
& \text { in } 20 \mu 1 \text { reaction buffer }
\end{aligned}
$$

After incubation for $30 \mathrm{~min}$ at $37^{\circ} \mathrm{C}$ (or the temperature optimal for the particular enzyme), $4 \mu \mathrm{l}$ of $5 \mathrm{x}$ loading dye was added to the mixture and the reaction products were analyzed on an agarose gel.

\section{Dephosphorylation of linear DNA fragments}

5'-end phosphate groups can be cleaved off with the aid of the Antarctic Phosphatase (New England Biolabs). Since DNA strands cannot ligate without 5'-end phosphate groups, a religation of linearized plasmids can be prevented. A typical reaction mixture contained:

$1 \mu \mathrm{g}$ of linearized plasmid DNA

1 U Antarctic Phosphatase

in $100 \mu \mathrm{l}$ of Antarctic Phosphatase Buffer

The mixture was incubated for $30 \mathrm{~min}$ at $37^{\circ} \mathrm{C}$ and heat inactivated for $10 \mathrm{~min}$ at $65^{\circ} \mathrm{C}$. The linearized plasmid was purified using the QIAquick PCR Purification Kit (Qiagen).

\section{Blunting of 5'-end overhanging DNA Fragments}

The Taq polymerase is capable to fill up 5'-end overhanging DNA fragment ends in the presence of deoxynucleotides and magnesium ions to produce blunt ends. This approach is the method of choice to ligate incompatible fragment ends. A typical reaction mixture contained:

$1 \mu \mathrm{g}$ of DNA fragments

$50 \mu \mathrm{M}$ dNTPs

$1 \mathrm{U}$ Taq polymerase

in $50 \mu 1$ of Taq PCR buffer 
The mixture was incubated for $30 \mathrm{~min}$ at $72^{\circ} \mathrm{C}$. Treated DNA fragments can be used directly in a ligation reaction.

\section{Ligation of DNA fragments}

Double-stranded DNA fragments were covalently linked by T4 DNA ligase. The fragment was used in 5-fold molar excess over the dephosphorylated, linearized plasmid DNA. A typical reaction mixture contained:

100 ng linearized, dephosphorylated vector

5-fold molar excess of the linear fragment

$1 \mathrm{U}$ T4 DNA ligase

in $10 \mu 1 \mathrm{~T} 4$ ligase buffer

The mixture was incubated either at room temperature for about $6 \mathrm{~h}$ or at $16^{\circ} \mathrm{C}$ overnight. Plasmids were transformed in E. coli cells. Plasmid DNA was isolated from transformants and was restriction digested. DNA fragments were analyzed on an agarose gel.

\subsubsection{Gel electrophoresis of nucleic acids}

\section{Nondenaturing agarose gel electrophoresis}

Nucleic acids can be separated, identified and isolated with an agarose gel in an electric field according to their size (Sambrook et al., 1989). In general, agarose gels contain between 0.8 and $2 \%$ agarose. For this purpose, the appropriate amount of agarose was boiled in 0.5x TBE buffer. Ethidium bromide was added after cooling to about $60^{\circ} \mathrm{C}$ (final concentration $0.5 \mu \mathrm{g} / \mathrm{ml}$ ) and poured into an electrophoresis slide. After solidification of the gel, the gel chamber was filled with 0.5x TBE buffer. Samples were mixed in a 6:1 ratio with loading dye before they were loaded into the wells of the gel. Electrophoresis was performed at constant voltage $(5-10 \mathrm{~V} / \mathrm{cm})$. The DNA bands could be detected with UV transmitted light (wavelength $254 \mathrm{~nm}$ ) and a GelJet Imager 2004 (Intas).

5x TBE buffer
$500 \mathrm{mM}$ Tris-Borat ( $\mathrm{pH} \mathrm{7.9)}$
$10 \mathrm{mM} \mathrm{Na}$-EDTA
in $\mathrm{H}_{2} \mathrm{O}_{\text {bid }}$

\section{6x loading dye}

$50 \%(\mathrm{w} / \mathrm{v})$ sucrose

$0.25 \%(\mathrm{w} / \mathrm{v})$ bromophenol blue

$0.25 \%(\mathrm{w} / \mathrm{v})$ xylene cyanol FF

in TE buffer 


\subsubsection{Transfer and detection of DNA on membranes (Southern-Blot)}

For transfer and detection of DNA on membranes, genomic DNA was digested with restriction enzymes and separated in a $0.8 \%$ agarose gel $(2.5 \mathrm{~h}, 90 \mathrm{~V})$. Before transfer, the agarose gel was incubated in 0.25 $\mathrm{M} \mathrm{HCl}$ for 20 minutes to cleave some of the purine bases in the DNA fragment. This facilitates the transfer of large DNA fragments. Subsequently, the gel was washed for 20 min in DENAT solution and further for 20 min in RENAT solution. Transfer to a nylon membrane (Roti-Nylon plus, Carl Roth) was performed by capillary blot method. Thus, the transfer solution (20x SSC buffer) was taken up from a buffer reservoir via capillary forces through the gel into a stack of paper towels placed on the gel. The DNA fragments are released vertically from the gel by the buffer stream and bind to the overlying positively charged nylon membrane. An evenly distributed weight on the paper stack guarantees a tight connection between the respective layers of the transfer system. The Southern-Blot was set up in the following order from bottom to top:

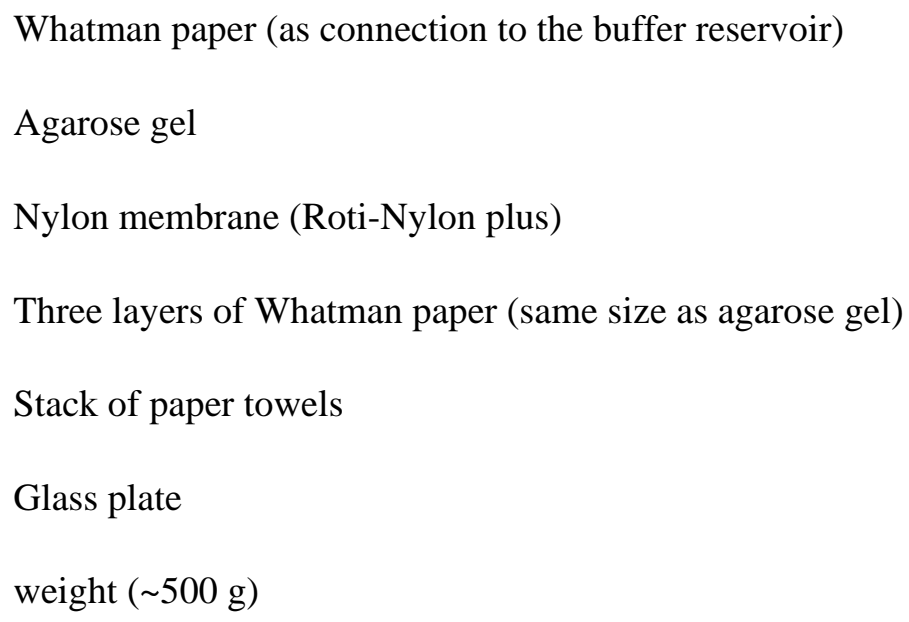

The Southern-Blot was performed overnight. Subsequently, the membrane was dried at room temperature and the DNA was fixed on the membrane by ultraviolet radiation (UV table for 1 min each side).

\section{Digoxigenin-labeled probe}

For the preparation of the probe, $6.6 \mu \mathrm{l}$ (maximal DNA amount of $1.5 \mu \mathrm{g}$ ) of the amplified flanking regions $(\mathrm{LB}+\mathrm{RB})$ of the gene to be examined was denatured for $10 \mathrm{~min}$ at $99^{\circ} \mathrm{C}$. The following components were added after cooling on ice:

$1 \mu 1$ 10x labeling buffer (Random Primer 6 (NEB, S1230S) in 10x buffer for Klenow fragment $1 \mu 1$ 10x BSA

$1 \mu \mathrm{l}$ 10x DIG-dNTP mix (1 mM dATP, dCTP, dGTP, 0.65 mM dTTP and 0.35 mM DIGdUTP)

$0.5 \mu 1$ Klenow fragment (Thermo Scientific) 
This reaction was incubated for $1 \mathrm{~h}$ or overnight at $37^{\circ} \mathrm{C}$ and then used for the specific detection of immobilized nucleic acids.

\section{Specific detection of immobilized nucleic acids}

The nylon membrane with the transferred immobilized nucleic acids was preincubated for $20 \mathrm{~min}$ at $65^{\circ} \mathrm{C}$ with Southern-hybridization buffer. Meanwhile, the probe was dissolved in $15 \mathrm{ml}$ Southern hybridization buffer and denatured at $95^{\circ} \mathrm{C}$ for $10 \mathrm{~min}$ in the water bath. The hybridization of the probe was performed at $65^{\circ} \mathrm{C}$ overnight in the hybridization oven. Subsequently, hybridization was followed by several washes for $15 \mathrm{~min}$ each at $65^{\circ} \mathrm{C}$ with $20 \mathrm{ml}$ 2x SSPE, 1x SSPE, 0.1x SSPE, respectively, and 5 min with DIG wash buffer at room temperature. The membrane was blocked with DIG2 buffer for 30 minutes to avoid non-specific binding of the anti-DIG antibody to the membrane. Thus, the membrane was incubated for $30 \mathrm{~min}$ with $10 \mathrm{ml}$ of anti-digoxigenin-AP antibody solution (1:10000 in DIG2). The membrane was washed twice with $50 \mathrm{ml}$ of DIG wash buffer for 15 min and equilibrated with $40 \mathrm{ml}$ DIG3 buffer for $5 \mathrm{~min}$. After incubation for 5 minutes with $10 \mathrm{ml}$ of CDP-Star® solution (1:500 in DIG3, New England Biolabs), the chemiluminescence was detected with a chemiluminescence imaging system (Peqlab). The exposure time was between $10 \mathrm{~s}$ and $15 \mathrm{~min}$.

\begin{tabular}{|c|c|}
\hline $\mathrm{HCl}$ & DIG1 \\
\hline $0.25 \mathrm{M} \mathrm{HCl}(37 \%(w / v))$ & $0.1 \mathrm{M}$ maleic acid \\
\hline \multirow[t]{2}{*}{ in $\mathrm{H}_{2} \mathrm{O}_{\text {bid }}$} & $0.15 \mathrm{M} \mathrm{NaCl}$ \\
\hline & in $\mathrm{H}_{2} \mathrm{O}_{\text {bid }}(\mathrm{pH} 7.5)$ \\
\hline \multicolumn{2}{|l|}{ DENAT solution } \\
\hline $1.5 \mathrm{M} \mathrm{NaCl}$ & DIG2 \\
\hline $0.4 \mathrm{M} \mathrm{NaOH}$ & $5 \%(\mathrm{w} / \mathrm{v})$ skimmed milk powder \\
\hline in $\mathrm{H}_{2} \mathrm{O}_{\text {bid }}$ & in DIG1 (see above) \\
\hline RENAT solution & DIG3 \\
\hline $1.5 \mathrm{M} \mathrm{NaCl}$ & $0.1 \mathrm{M}$ maleic acid \\
\hline $282 \mathrm{mM}$ Tris- $\mathrm{HCl}$ & $0.1 \mathrm{M} \mathrm{NaCl}$ \\
\hline $218 \mathrm{mM}$ Tris & $0.05 \mathrm{M} \mathrm{MgCl}_{2}$ \\
\hline in $\mathrm{H}_{2} \mathrm{O}_{\text {bid }}$ & in $\mathrm{H}_{2} \mathrm{O}(\mathrm{pH} 9.5)$ \\
\hline 20x SSC buffer & DIG wash buffer \\
\hline $3 \mathrm{M} \mathrm{NaCl}$ & $0.3 \%(\mathrm{v} / \mathrm{v})$ Tween-20 in DIG 1 (see below) \\
\hline \multicolumn{2}{|l|}{$300 \mathrm{mM}$ sodium citrate } \\
\hline \multirow[t]{3}{*}{ in $\mathrm{H}_{2} \mathrm{O}_{\text {bid }}(\mathrm{pH} 7.4)$} & Na-phosphate buffer (1 M) \\
\hline & Solution 1: \\
\hline & $1 \mathrm{M} \mathrm{Na} 2 \mathrm{HPO} 4$ x $2 \mathrm{H} 2 \mathrm{O}$ \\
\hline 20x SSPE buffer & in $\mathrm{H}_{2} \mathrm{O}_{\text {bid }}$ \\
\hline $3 \mathrm{M} \mathrm{NaCl}$ & \\
\hline
\end{tabular}




$$
\begin{aligned}
& 227 \mathrm{mM} \mathrm{Na}_{2} \mathrm{PO}_{4} \times \mathrm{H}_{2} \mathrm{O} \\
& 20 \mathrm{mM} \mathrm{Na} \text {-EDTA } \times 2 \mathrm{H}_{2} \mathrm{O} \\
& \text { in } \mathrm{H}_{2} \mathrm{O}_{\text {bid }}(\mathrm{pH} \mathrm{7.4})
\end{aligned}
$$

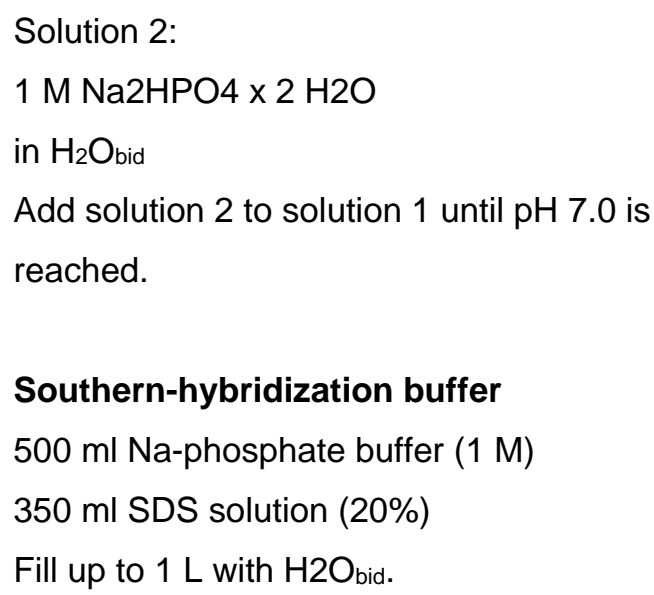

\subsubsection{Polymerase chain reaction (PCR)}

The method is modified according to Innis (1990). For the amplification of DNA fragments the Phusion DNA polymerase or for advanced applications the Q5 polymerase (New England Biolabs) was used. In general, the reactions were performed with a hot-start in a volume of $50 \mu \mathrm{l}$. The PCR reaction starts with an initial denaturation, followed by approximately 30 cycles of denaturation, annealing and elongation. In the end, a final elongation of 2 min or 5 min was performed. The elongation time was determined depending on the size of the expected PCR product and the polymerase which was used. The annealing temperatures were mostly $60^{\circ} \mathrm{C}$ but were modified if necessary. The reactions took place in the thermocycler T100 from Bio-Rad.

\section{PCR reaction with Phusion or Q5 polymerase}

10 ng template DNA

$200 \mu \mathrm{M}$ dNTPs

$1 \mu \mathrm{M}$ Oligonucleotide 1

$1 \mu \mathrm{M}$ Oligonucleotide 2

1 x HF buffer (Thermo Fisher Scientific) or Q5 buffer (New England Biolabs)

1 U Phusion polymerase or Q5 polymerase (New England Biolabs)

in $\mathrm{H}_{2} \mathrm{O}_{\text {bid }}$

\section{Program Phusion}

$98^{\circ} \mathrm{C} / \infty-98^{\circ} \mathrm{C} / 30 \mathrm{~s}-\left(98^{\circ} \mathrm{C} / 10 \mathrm{~s}-58-68^{\circ} \mathrm{C} / 15 \mathrm{~s}-72^{\circ} \mathrm{C} / \mathrm{x} \min \right)$ x $30-72^{\circ} \mathrm{C} / 5 \mathrm{~min}-12^{\circ} \mathrm{C} / \infty$ 


\section{Program Q5}

$98^{\circ} \mathrm{C} / \infty-98^{\circ} \mathrm{C} / 30 \mathrm{~s}-\left(98^{\circ} \mathrm{C} / 10 \mathrm{~s}-58-68^{\circ} \mathrm{C} / 15 \mathrm{~s}-72^{\circ} \mathrm{C} / \mathrm{x} \min \right) \times 30-72^{\circ} \mathrm{C} / 2 \mathrm{~min}-12^{\circ} \mathrm{C} / \infty$

\subsubsection{Quantitative reverse transcription-PCR (qRT-PCR)}

\section{DNase treatment and cDNA synthesis}

The TURBO DNA-free kit (Ambion) was used for the DNase treatment of the isolated total RNA. The RNA quality was previously checked by agarose gel electrophoresis and the concentration was determined at a wavelength of $260 \mathrm{~nm}$ with the nanodrop. The reaction mixture contained:

$$
4.25 \mu \mathrm{g} \text { RA }
$$

$0.75 \mu 1$ Turbo DNase

$2.5 \mu \mathrm{l}$ buffer

In $21.5 \mu \mathrm{l} \mathrm{H} \mathrm{H}_{2} \mathrm{O}$ RNase free water (Ambion)

After 30 min incubation at $37^{\circ} \mathrm{C}$, the reaction was terminated by addition of $2.5 \mu$ of DNase inactivation and incubated for 5 min at RT. After centrifugation (2 min, 10000 rpm, Heraeus Pico 17), the supernatant was removed and used for cDNA synthesis.

For cDNA synthesis, the RevertAid First Strand cDNA Synthesis Kit (Thermo Fisher Scientific) was used according to the manufacturer's instructions. Thus, $6 \mu \mathrm{l}$ of DNase digested RNA was used. For long-term storage, samples were stored at $-80^{\circ} \mathrm{C}$. The qRT-PCR reactions were performed in a Bio-Rad CFX Connect.

\section{qRT-PCR reaction}

$1 \mu \mathrm{lDNA}$

$5 \mu 1$ 2x MESA GREEN qPCR Master Mix (Eurogentech)

$2 \mu 1$ Primer Mix (each primer 2 pmol)

$2 \mu \mathrm{l}$ nuclease-free $\mathrm{H}_{2} \mathrm{O}$ (Ambion)

\section{qRT-PCR Programm}

$95^{\circ} \mathrm{C} / 5 \min -\left(98^{\circ} \mathrm{C} / 15 \mathrm{~s}-62^{\circ} \mathrm{C} / 20 \mathrm{~s}-72^{\circ} \mathrm{C} / 40 \mathrm{~s}\right)$ x 37 - Melting curve: $72^{\circ} \mathrm{C}-95^{\circ} \mathrm{C}$ 


\subsection{Genetic methods}

\subsubsection{PCR amplification of gene deletion and fusion constructs for $\boldsymbol{U}$. maydis}

Gene deletions and gene fusions were performed according to Kämper (2004). The strategy is based on homologous recombination by replacing the open reading frame of a gene (ORF) with a resistance cassette. Thus, gene deletion or fusion constructs were transformed into U. maydis containing the resistance cassette and 1000 bp of the homologous regions (LB and RB) flanking the gene. The homologous regions of the genome were amplified by PCR and SfiI restriction sites were inserted via the primers. The resistance cassette and flanking gene borders were restriction digested with SfiI and ligated overnight at $16^{\circ} \mathrm{C}$. The ligation product was isolated via an agarose gel (QIAquick Gel Extraction Kit) and cloned via topo cloning in the vector pCR2.1 (Invitrogen). All resistance cassettes used are listed in Brachmann et al. (2004).

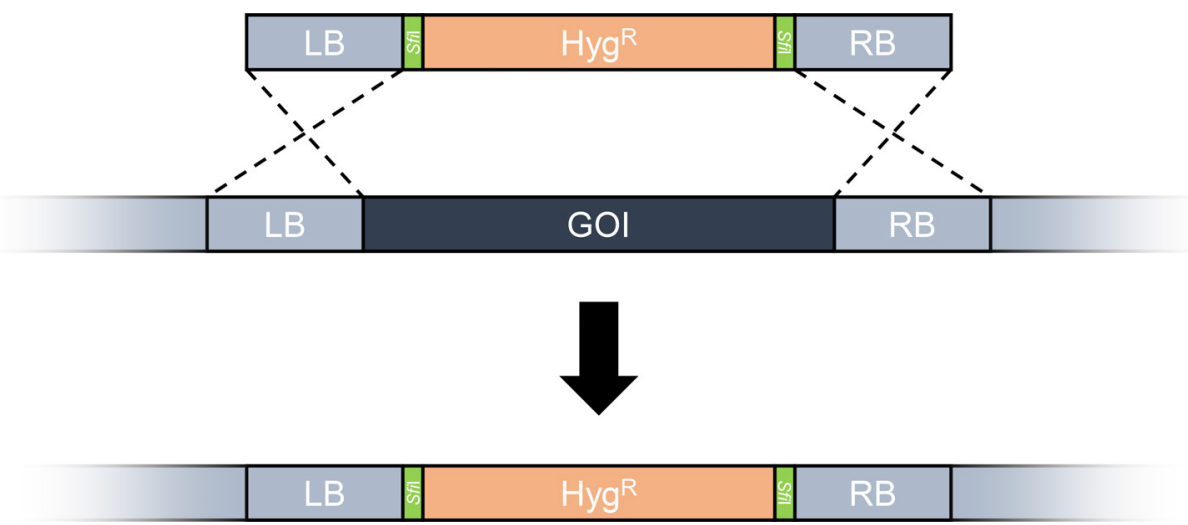

Figure 5.1: Schematic representation of the gene deletion by homologous recombination. The deletion construct consists of a homologous region (LB and RB), SfiI restriction sites (green) and the resistance cassette (hygromycin $\left(\mathrm{Hyg}^{\mathrm{R}}\right.$ ) as an example). The gene of interest (GOI) is replaced after the transformation of the gene deletion construct by homologous recombination.

\subsubsection{Integration of constructs in the ip locus of $U$. maydis}

The substitution of histidine for leucine (A257S) in the succinate dehydrogenase (sdh1; UMAG_01172) of $U$. maydis leads to resistance to the antibiotic carboxin (Broomfield and Hargreaves, 1992). This can be used to target constructs into the sdh1 locus (ip (iron-sulfur protein) locus) of U. maydis. Thus, plasmids contain the mutated variant of $s d h 1\left(i p^{R}\right)$ and the gene of interest with its own promoter or an overexpression promoter. The linearized plasmid (usually linearized with $S s p \mathrm{I}$ ) are transformed into $U$. maydis and integrated into the native locus $\left(i p^{S}\right)$ by homologous recombination. The integrated construct is then flanked by the mutated $\left(i p^{R}\right)$ and the native $s d h 1$ variant $\left(i p^{S}\right)$. Integration of the plasmid can occur once (single) or multiple times in the ip locus, which can be examined by Southern-Blot. 


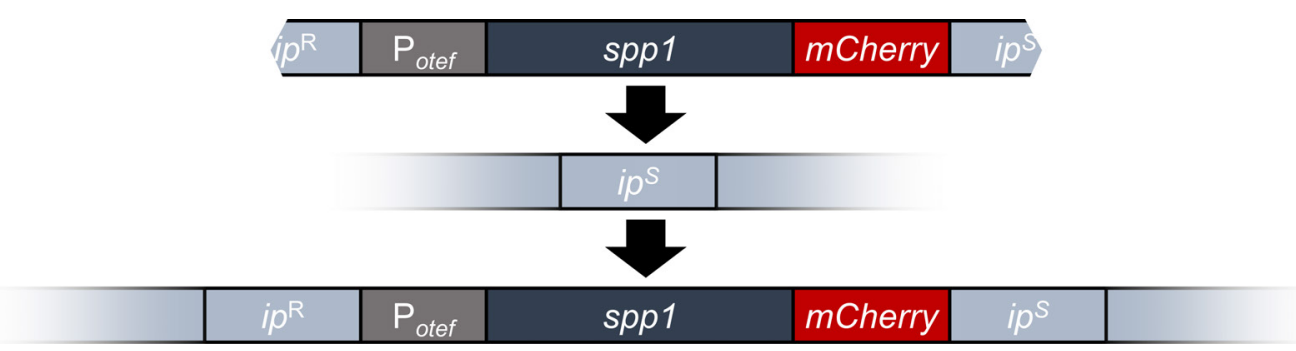

Figure 5.2: Schematic representation of the ectopic integration of constructs in the ip locus. The plasmid to be integrated is flanked by the mutated $i p^{\mathrm{R}}$ and the $s d h 1\left(i p^{\mathrm{S}}\right)$ gene. After linearization of the plasmid, the construct is integrated into the native $i p^{\mathrm{S}}$ locus by homologous recombination. Constructs can be integrated into again in the native $i p^{\mathrm{S}}$ locus resulting in multiple integrations of the constructs. Depicted is the spp1-mC fusion construct under expression of the otef promoter $\left(\mathrm{P}_{\text {otef }}\right)$.

\subsection{Biochemical methods}

\subsubsection{Protein extraction of $U$. maydis for protein analyses}

\section{Standard protein extraction}

To prepare protein extracts, $15 \mathrm{ml}$ of a CM liquid culture were centrifuged (5 min, $3500 \mathrm{rpm}, 4^{\circ} \mathrm{C}$, Heraeus Biofuge Stratos, rotor \#3047). The pellet was washed once with ice-cold TBS buffer (5 min, $3500 \mathrm{rpm}, 4^{\circ} \mathrm{C}$, Heraeus Biofuge Stratos, rotor \#3047). Subsequently, the pellet was resuspended in 300 $\mu \mathrm{l}$ of TBS+ lysis buffer and transferred into a $2 \mathrm{ml}$ reaction vessel with $300 \mathrm{mg}$ glass beads. The samples were flash frozen in liquid nitrogen and stored at $-80^{\circ} \mathrm{C}$. The cells were disrupted for 30 min at $4{ }^{\circ} \mathrm{C}$ on the Vibrax VRX (IKA) at $1500 \mathrm{rpm}$. Finally, the samples were centrifuged $\left(13000 \mathrm{rpm}, 10 \mathrm{~min}, 4^{\circ} \mathrm{C}\right.$, Heraeus Biofuge Stratos, rotor \#3331) and the supernatant was transferred to a new reaction vessel. For long-term storage, the samples were flash-frozen and stored at $-80^{\circ} \mathrm{C}$. After determining the protein concentration via the Nanodrop ND-1000, the samples were adjusted to the desired concentration with $\mathrm{H}_{2} \mathrm{O}$. For further use in SDS gel electrophoresis, SDS sample buffer (Roti Load 1, Carl Roth) was added in a 1:4 ratio and samples were boiled for $3 \mathrm{~min}$ at $98^{\circ} \mathrm{C}$.

\author{
TBS buffer \\ $50 \mathrm{mM}$ Tris $\mathrm{HCl}(\mathrm{pH} 7.5)$ \\ $150 \mathrm{mM} \mathrm{NaCl}$
}

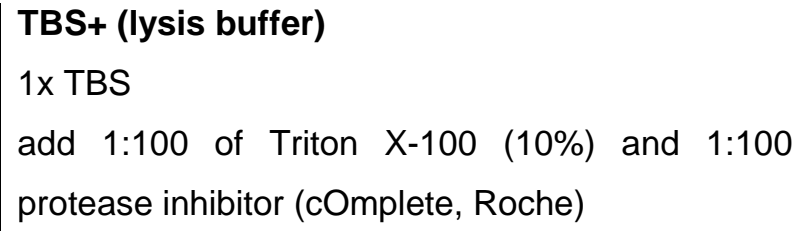

\section{Isolation of proteins of culture supernatant of $U$. maydis}

For secretion assays of Pit2-mC, Pep1-mC, Tin2-mC and Cmu1-mC, strains were inoculated for an over day culture in $\mathrm{CM}$ liquid medium and incubated shaking at $28^{\circ} \mathrm{C}$. In the evening, strains were adjusted to an $\mathrm{OD}_{600}$ of 0.35 for the next day. After growth overnight, $\mathrm{OD}_{600}$ was checked and the UPR was activated (5 $\mu \mathrm{g} / \mathrm{ml}$ f.c.) for $4 \mathrm{~h}$ at $28^{\circ} \mathrm{C}$. After UPR activation, $10 \mathrm{ml}$ of the cultures were collected and 
centrifuged (5 min, $3500 \mathrm{rpm}, 4^{\circ} \mathrm{C}$, Heraeus Biofuge Stratos, rotor \#3047) to obtain the pellet fraction. The pellet was subjected to the standard protein extraction method. The remaining $40 \mathrm{ml}$ of the culture was centrifuged (10 min, $3500 \mathrm{rpm}, 4^{\circ} \mathrm{C}$, Heraeus Biofuge Stratos, rotor \#3047) and $30 \mathrm{ml}$ supernatant was transferred into a new $50 \mathrm{ml}$ reaction vessel and stored on ice. Subsequently, each supernatant was sterile filtered with a $20 \mathrm{ml}$ syringe and a sterile filter ( $0.2 \mu \mathrm{m}$ pore size, Sarstedt) into a Vivaspin sample concentrator with a molecular weight cut-off of $10 \mathrm{kDa}$ (28-9323-60, Sartorius). After centrifugation (35 min, $5000 \mathrm{~g}, 4^{\circ} \mathrm{C}$, Heraeus Biofuge Stratos, rotor \#3047), $200 \mu \mathrm{l}$ of concentrated supernatant was collected and transferred into a fresh $1.5 \mathrm{ml}$ reaction vessel. $30 \mu \mathrm{l}$ of supernatant was boiled with $10 \mu \mathrm{l}$ SDS sample buffer (Roti Load 1, Carl Roth) at $98^{\circ} \mathrm{C}$ for $3 \mathrm{~min}$. Samples of the pellet and the supernatant fraction were subjected to SDS-PAGE and western-hybridization for further analysis.

\section{Protein extraction of Spp1-GFP/ Spp1 ${ }^{\mathrm{D} 279 \mathrm{~A}}-$ GFP for Western hybridization}

Cell pellets were resuspended in $300 \mu \mathrm{TBS}$ (supplemented with 1x cOmplete proteinase inhibitor mix (Roche) and 2\% (w/v) digitonin (4005, Carl Roth) and transferred into $2 \mathrm{ml}$ reaction vessel with $300 \mathrm{mg}$ glass beads. Samples were flash-frozen in liquid nitrogen and stored at $-80^{\circ} \mathrm{C}$. The cells were disrupted for $30 \mathrm{~min}$ at $4^{\circ} \mathrm{C}$ on the Vibrax VRX (IKA) at $1500 \mathrm{rpm}$. Subsequently, the samples were centrifuged (2 min, $1200 \mathrm{~g}, 4^{\circ} \mathrm{C}$, Heraeus Biofuge Stratos, rotor \#3331), to enrich the supernatant with ER membranes. The supernatant was transferred to a new $1.5 \mathrm{ml}$ reaction vessel and centrifuged again (20 min, $22000 \mathrm{rpm}, 4^{\circ} \mathrm{C}$, Heraeus Biofuge Stratos, rotor \#3331). Finally, $10 \mu \mathrm{l}$ of SDS sample buffer (Roti Load 1, Carl Roth) was added to $30 \mu \mathrm{l}$ of the supernatant, incubated for $10 \mathrm{~min}$ at $65^{\circ} \mathrm{C}$ and stored at $-20^{\circ} \mathrm{C}$ until Western hybridization.

\subsubsection{SDS polyacrylamide gel electrophoreses of proteins}

The discontinuous SDS-polyacrylamide gel electrophoresis (SDS-PAGE) was performed after Laemmli (1970), in combination with the Mini-PROTEAN Tetra Handcast System (Bio-Rad). The separation of proteins was performed at $100 \mathrm{~V}$ until the blue loading dye band of the SDS sample buffer reached the end of the separation gel. For a size standard in the protein gels, the PageRuler Prestained Protein Ladder (10 to $180 \mathrm{kDa}$, 26616, Thermo Fisher Scientific) was used. For detection of protein bands, the gels were fixed for $60 \mathrm{~min}$ (fixing solution) and stained for $3 \mathrm{~h}$ in Coomassie staining solution. After staining, the gels were decolorized with fixing solution for $1 \mathrm{~h}$ and further decolorized in $\mathrm{H}_{2} \mathrm{O}$, with a piece of white paper towel overnight.

\author{
Stacking gel \\ $125 \mathrm{mM}$ Tris- $\mathrm{Cl}(\mathrm{pH} 6.8)$ \\ $4 \%(w / v)$ acrylamide mix \\ $0.1 \%(w / v)$ SDS \\ $0.05 \%(\mathrm{w} / \mathrm{v})$ ammonium persulfate
}

\author{
Protein gel running buffer \\ $25 \mathrm{mM}$ Tris-Cl \\ $250 \mathrm{mM}$ glycine \\ $0.1 \%(w / v)$ SDS \\ in $\mathrm{H}_{2} \mathrm{O}_{\text {bid }}(\mathrm{pH} 8.8)$
}


$0.1 \%(v / v)$ TEMED

\section{Separating gel}

375 mM Tris- $\mathrm{Cl}$ (pH 8.8)

8-12\% acrylamide mix

$0.1 \%(w / v)$ SDS

$0.05 \%(\mathrm{w} / \mathrm{v})$ ammonium persulfate

$0.1 \%(v / v)$ TEMED

\section{Fixing solution}

$40 \%(\mathrm{v} / \mathrm{v})$ ethanol

$10 \%(\mathrm{v} / \mathrm{v})$ acetic acid

in $\mathrm{H}_{2} \mathrm{O}_{\text {bid }}$

\section{Coomassie staining solution}

$0.1 \%(w / v)$ SERVA blue R (SERVA)

$45 \%(\mathrm{v} / \mathrm{v})$ ethanol

$10(\mathrm{v} / \mathrm{v})$ acetic acid

in $\mathrm{H}_{2} \mathrm{O}$ bid

\subsubsection{Detection of immobilized proteins (Western-Blot)}

To transfer proteins from SDS-polyacrylamide gels to a PVDF membrane (Amersham Hybond P 0.45 PVDF, GE Healthcare), a Semi-Dry Electroblotter (Peqlab) or the Trans-Blot Turbo system (Bio-Rad) was used. For western-hybridization with the system of peqlab, the membrane was activated in methanol (Standard) for $1 \mathrm{~min}$ and then equilibrated in Western transfer buffer. The SDS-polyacrylamide gel was also equilibrated in Western transfer buffer. The blotting of proteins was performed for $2 \mathrm{~h}$ at $75 \mathrm{~mA}$ per SDS gel. Western-hybridization with the Bio-Rad system was performed according to the manufacturer's manual. The blot (with peqlab system) was set up as followed from top to bottom:

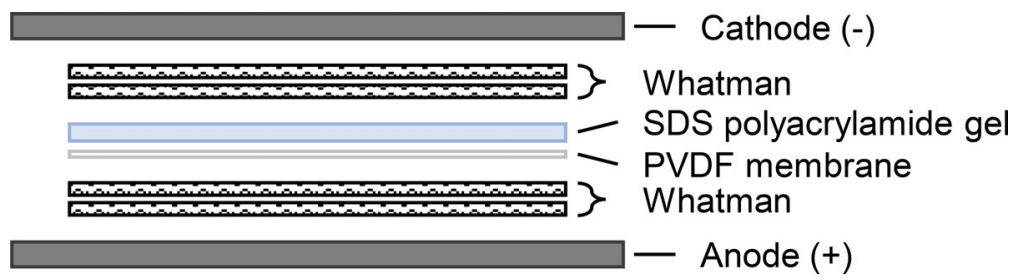

Subsequently, the membrane was shaken for $30 \mathrm{~min}$ in blocking solution to prevent nonspecific binding of the antibody. Incubation of the primary antibody was performed overnight at $4^{\circ} \mathrm{C}$. On the next day, the membrane was washed three times with TBS-T for 5 minutes and incubated with the secondary antibody for 1-2 hours at RT. Subsequently, the membrane was washed three times for 5 min with TBS$\mathrm{T}$ and once for 5 min with TBS. For the development of the membrane, it was incubated with $1 \mathrm{ml}$ Luminata Crescendo Western HRP Substrate (Millipore). The signals were detected with a chemiluminescence imaging system (Peqlab) and evaluated with ImageJ 1.48 (https://imagej.nih.gov/ij). To stain protein bands, the membrane was incubated with Ponceau S staining solution for 20 min and washed three times with $\mathrm{H}_{2} \mathrm{O}$.

TBS-T

$0.1 \%(v / v)$ Tween-20 in TBS

\section{Blocking solution}

$5 \%(\mathrm{w} / \mathrm{v})$ skim milk powder in TBS-T 


\author{
Western transfer buffer \\ 192 mM Glycine \\ $15 \%(\mathrm{v} / \mathrm{v})$ methanol \\ in $25 \mathrm{mM}$ Tris- $\mathrm{HCl}(\mathrm{pH} 10.4)$
}

\section{antibody solution}

$5 \%(\mathrm{w} / \mathrm{v})$ skim milk powder

in TBS-T with an appropriate antibody

\subsubsection{Stability assay of Cib1}

Stability of Cib1-GFP in response to Clp1 expression was determined with a doxycycline (DOX) based promoter shut-off system ( $\mathrm{P}_{\text {teto: }}$ cib1-GFP) (Zarnack et al., 2006). U. maydis strains were grown in CM supplemented with $1 \%$ glucose (CMG) to an $\mathrm{OD}_{600}$ of 0.35 and shifted to $\mathrm{CM}$ supplemented with $1 \%$ arabinose (CMA) to induce $\mathrm{P}_{\text {crg1 }}$-driven $c l p 1$ expression. For UPR activation, TM was added to a final concentration of $5 \mu \mathrm{g} / \mathrm{ml}$. $4 \mathrm{~h}$ after UPR induction DOX (10 $\mu \mathrm{g} / \mathrm{ml})$ was added (T0) and protein extract was prepared from samples taken at the time points $1 \mathrm{~h}$ (T1), $2 \mathrm{~h}$ (T2), $3 \mathrm{~h}$ (T3) and $4 \mathrm{~h}$ (T4) after DOX treatment. Cycloheximide (CHX)-based determination of Cib1-GFP was performed as described before (Heimel et al., 2013). Briefly, cells were grown as described for promoter shut-off assays. Protein biosynthesis was inhibited using CHX (100 $\mu \mathrm{g} / \mathrm{ml})$ and cells were sampled directly before (T0), or at the time points $30 \mathrm{~min}$ (T1), $60 \mathrm{~min}$ (T2) or $90 \mathrm{~min}$ (T3) after CHX treatment. Cib1-GFP levels were quantified using ImageJ 1.48 (https://imagej.nih.gov/ij) and normalized to Ponceau S stained bands. Stability of proteins was calculated relative to T0. Experiments were performed in three biological replicates. Statistical significances (P-value) were calculated using the Student’s $t$-test.

\subsubsection{On-bead phosphatase assay of Cib1}

The protein phosphatase assay was performed after immunoprecipitation of Cib1-GFP followed by onbead treatment with lambda-phosphatase (New England Biolabs). Cells were incubated as described for promoter shut-off experiments (Chapter 5.5.4). Briefly, $4 \mathrm{~h}$ after TM-mediated UPR activation ( $5 \mu \mathrm{g} / \mathrm{ml}$ ), equal culture volumes were centrifuged ( $5 \mathrm{~min}, 3500 \mathrm{rpm}, 4^{\circ} \mathrm{C}$, Heraeus Biofuge Stratos, rotor \#3047), washed once with TBS, supplemented with 2x cOmplete proteinase inhibitor (Roche), (PI) and 1x phosphatase inhibitor cocktail (PhI). The pellet was resuspended in $750 \mu$ l buffer B-300, flashfrozen in liquid nitrogen and disrupted in a cell mill (Retsch MM400, 30Hz, 2min). After cell lysis, 750 $\mu \mathrm{l}$ of $\mathrm{B}+300$, was added and the whole cell lysate was centrifuged (30 min, $22000 \mathrm{rpm}, 4^{\circ} \mathrm{C}$, Heraeus Biofuge Stratos, rotor \#3331). The supernatant was added to $60 \mu \mathrm{l}$ of magnetic agarose GFP-Trap beads (chromotek) and incubated for $3 \mathrm{~h}$ at $4^{\circ} \mathrm{C}$ on a rotating wheel. After washing the beads $2 \mathrm{x}$ with $500 \mu \mathrm{l}$ of B-300 buffer and removing the supernatant, beads were resuspended in $600 \mu \mathrm{l}$ of buffer B-300 (supplemented only with 2x PI, not with PhI) evenly distributed in $200 \mu$ laliquots. The supernatant was discarded and 1x lambda phosphatase buffer (NEB) was added to each sample. 1200U of lambda phosphatase were added. Control samples were left untreated or supplemented with $2 \mathrm{x}$ PhI. After incubation for $30 \mathrm{~min}$ at $30^{\circ} \mathrm{C}$, the supernatant was discarded and $30 \mu \mathrm{l} 1 \mathrm{x}$ Roti Load 1 (Carl-Roth) was 
added to the beads and boiled at $98^{\circ} \mathrm{C}$ for $3 \mathrm{~min}$. Samples were run on a $10 \%$ SDS-PAGE and subjected to Western hybridization. All steps were performed in Protein LoBind Tubes (Eppendorf). Experiments were repeated in three independent experiments.

Phosphatase inhibitor (Phl)
$1 \mathrm{mM} \mathrm{NaF}$
$0.5 \mathrm{mM} \mathrm{Na}_{3} \mathrm{VO}_{4}$
$8 \mathrm{mM} \beta$-glycerophosphate

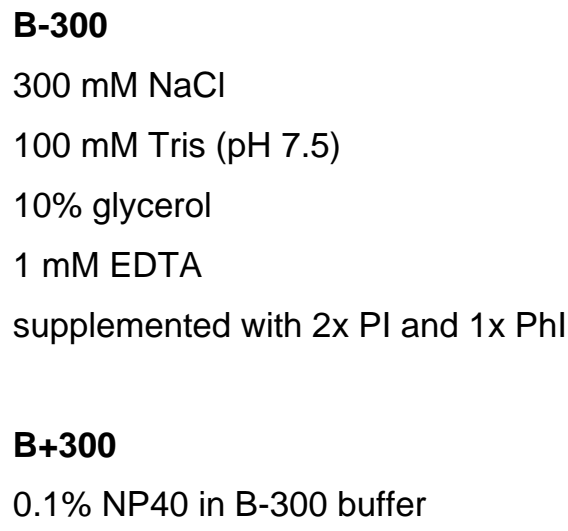

\subsubsection{Identification of Cib1 phosphosites}

The identification of Cib1 phosphosites was performed by immunoprecipitation of Cib1-GFP during absence and induction of Clp1. Cells were incubated as described for promoter shut-off experiments (Chapter 5.5.4). Briefly, $4 \mathrm{~h}$ after TM-mediated UPR activation (5 $\mu \mathrm{g} / \mathrm{ml})$, equal culture volumes were centrifuged (5 min, $3500 \mathrm{rpm}, 4^{\circ} \mathrm{C}$, Heraeus Biofuge Stratos, rotor \#3047), washed once with TBS, supplemented with 2x cOmplete proteinase inhibitor (Roche), (PI) and 1x phosphatase inhibitor cocktail (PhI). The pellet was resuspended in $750 \mu$ l buffer B-300, flash-frozen in liquid nitrogen and disrupted in a cell mill (Retsch MM400, 30Hz, 2min). After cell lysis, $750 \mu \mathrm{l}$ of B+300, was added and the whole cell lysate was centrifuged (30 min, $22000 \mathrm{rpm}, 4^{\circ} \mathrm{C}$, Heraeus Biofuge Stratos, rotor \#3331). The supernatant was added to $40 \mu \mathrm{l}$ of magnetic agarose GFP-Trap beads (chromotek) and incubated for 3 $\mathrm{h}$ at $4^{\circ} \mathrm{C}$ on a rotating wheel. After washing the beads $3 \mathrm{x}$ with $500 \mu \mathrm{l}$ of B-300 buffer and removing the supernatant, $30 \mu \mathrm{l} 1 \mathrm{x}$ Roti Load 1 (Carl-Roth) was added to the beads and boiled at $98^{\circ} \mathrm{C}$ for $3 \mathrm{~min}$. Subsequently, the supernatant was transferred into a new $1.5 \mathrm{ml}$ reaction vessel and samples were run on a $10 \%$ SDS-PAGE at $100 \mathrm{~V}$ until the blue loading dye of the SDS sample buffer ran out of the separation gel. Gels were fixed 60 min with fixing solution, stained $3 \mathrm{~h}$ with Coomassie staining solution and destained for $1 \mathrm{~h}$ with fixing solution (Chapter 5.5.2). Visible protein bands of Cib1-GFP were cut out and subjected to LC-MS analysis (Chapter 5.5.8, Orbitrap Velos Pro and Q Exactive HF).

\subsubsection{Immunoprecipitation of Spp1-GFP / Spp1 ${ }^{\mathrm{D} 279 \mathrm{~A}}$-GFP in U. maydis}

For immunoprecipitation of Spp1-GFP / Spp1 $1^{\mathrm{D} 279 \mathrm{~A}}-\mathrm{GFP}$, strains were grown to an $\mathrm{OD}_{600}$ of 0.35 and UPR was activated with $5 \mu \mathrm{g} / \mathrm{ml}$ TM for $4 \mathrm{~h}$. Subsequently, equal culture volumes were centrifuged (5 min, 3500 rpm, $4^{\circ} \mathrm{C}$, Heraeus Biofuge Stratos, rotor \#3047) and washed once with TBS (supplemented with 2x cOmplete proteinase inhibitor (PI, Roche)). The pellet was resuspended in $300 \mu 1$ of TBS 
(supplemented with 2x PI), transferred into a fresh $2 \mathrm{ml}$ reaction vessel containing $300 \mathrm{mg}$ glass beads. The samples were flash frozen in liquid nitrogen and stored at $-80^{\circ} \mathrm{C}$. The cells were disrupted for 30 $\min$ at $4^{\circ} \mathrm{C}$ on the Vibrax VRX (IKA) at $1500 \mathrm{rpm}$. Subsequently, the samples were centrifuged (2 min, $1200 \mathrm{~g}, 4^{\circ} \mathrm{C}$, Heraeus Biofuge Stratos, rotor \#3331), to enrich the supernatant with ER membranes. The supernatant was transferred to a new $1.5 \mathrm{ml}$ reaction vessel and centrifuged again (20 min, $22000 \mathrm{rpm}$, $4^{\circ} \mathrm{C}$, Heraeus Biofuge Stratos, rotor \#3331). The supernatant was removed completely and the pellet dissolved in $100 \mu \mathrm{l}$ of TBS supplemented with $2 \%$ (w/v) digitonin (4005, Carl Roth) and 1x PI. Digitonin permeabilizes ER membranes and supports the release of ER membrane proteins (Baghirova et al., 2015). For Western hybridization of Spp1-GFP / Spp1 $1^{\mathrm{D} 279 \mathrm{~A}}-\mathrm{GFP}, 10 \mu \mathrm{l}$ of SDS sample buffer (Roti Load 1, Carl Roth) was added to $30 \mu \mathrm{l}$ of the samples and incubated for $10 \mathrm{~min}$ at $65^{\circ} \mathrm{C}$. The remaining $70 \mu \mathrm{l}$ of the samples were filled up to $500 \mu \mathrm{l}$ with TBS (supplemented with $2 \%$ (w/v) digitonin and $1 \mathrm{x}$ PI). The sample was transferred to a fresh $1.5 \mathrm{ml}$ reaction vessel with $15 \mu \mathrm{l}$ of washed magnetic agarose GFP-Trap beads (washed 3 times in $500 \mu \mathrm{l}$ 1x TBS supplemented with 1x PI) and incubated for $3 \mathrm{~h}$ at $4^{\circ} \mathrm{C}$ on a rotating wheel. Subsequently, GFP trap beads were washed $7 \mathrm{x}$ with $500 \mu \mathrm{TBS}$ (supplemented with $0.1 \%$ of digitonin and $1 \mathrm{x}$ PI). Finally, $50 \mu \mathrm{l}$ of TBS (supplemented with $0.1 \%$ of digitonin and $1 \mathrm{x}$ PI) was added to the beads as well as $16.6 \mu \mathrm{l}$ of SDS sample buffer (Roti Load 1, Carl Roth) and denatured for $3 \mathrm{~min}$ at $95^{\circ} \mathrm{C}$. Subsequently, the supernatant was transferred into a new $1.5 \mathrm{ml}$ reaction vessel and samples were run on a 10\% SDS-PAGE at $100 \mathrm{~V}$ until the blue loading dye of the SDS sample buffer ran $10 \mathrm{~mm}$ into the separation gel. Lanes were cut out $(10 \mathrm{~mm})$ and gel pieces were fixed 60 min with fixing solution and subjected to LC-MS analysis (Chapter 5.5.8, Orbitrap Velos Pro).

\subsubsection{Mass spectroscopic analyses (LC-MS) \\ RSLCnano Ultimate 3000 system and Orbitrap Velos Pro}

The cutout gel lanes were each cut into 10 pieces and subjected to tryptic digestion according to Shevchenko et al. (1996) followed by LC-MS analysis. Subsequently, peptides were purified with C18 stop and go extraction (stage) tips according to Rappsilber et al. (2003) and Rappsilber et al. (2007). The resulting peptide solution was dried completely in the SpeedVac concentrator and resolved in the sample buffer ( $2 \%$ acetonitrile, $0.1 \%$ formic acid) for LC-MS analyses. LC-MS analysis for protein identification was performed with an Orbitrap Velos ProTM Hybrid Ion Trap-Orbitrap mass spectrometer. $4 \mu \mathrm{l}$ of peptide solutions were loaded and washed on an Acclaim ${ }^{\circledR}$ PepMAP 100 pre-

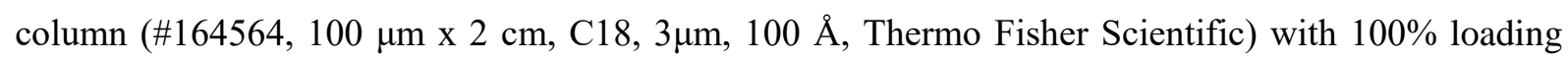
solvent A ( $2 \%$ acetonitrile, $0.07 \%$ trifluoroacetic acid) at a flow rate of $25 \mu 1 / \mathrm{min}$ for $6 \mathrm{~min}$. Peptides were separated by reverse phase chromatography on an Acclaim ${ }^{\circledR}$ PepMAP RSLC column ( $75 \mu \mathrm{m}$ x 50 cm (\#164540), C18, $3 \mu \mathrm{m}, 100 \AA$, Thermo Fisher Scientific) with a gradient from 98\% solvent A $(0.1 \%$ formic acid) and 2\% solvent B (80\% acetonitrile, 0.1\% formic acid) to 65\% solvent B in 121 min and subsequently to $98 \%$ solvent B in 1 min followed by $16 \mathrm{~min}$ at $98 \%$ solvent $\mathrm{B}$. The flow rate for the 
gradient was $300 \mathrm{nl} / \mathrm{min}$. Peptides eluting from the chromatographic column were on-line ionized by nanoelectrospray at $2.4 \mathrm{kV}$ with the Nanospray Flex Ion Source (Thermo Fisher Scientific). Full scans of the ionized peptides were recorded within the Orbitrap FT analyzer of the mass spectrometer within a mass range of $300-1850 \mathrm{~m} / \mathrm{z}$ at a resolution of 30,000 . Collision-induced dissociation (CID) fragmentation of data-dependent top-ten peptides was performed with the LTQ Velos Pro linear ion trap. For data acquisition and programming, the XCalibur 2.2 software (Thermo Fisher Scientific) was used.

\section{RSLCnano Ultimate 3000 system and Q Exactive HF}

The cutout gel lanes were each cut into 10 pieces and subjected to tryptic digestion according to Shevchenko et al. (1996) followed by LC-MS analysis. Subsequently, peptides were purified with C18 stop and go extraction (stage) tips according to Rappsilber et al. (2003) and Rappsilber et al. (2007) and analyzed with nanoflow LC coupled to nano ESI mass spectrometry. Nano LC - RSLCnano Ultimate 3000 system (Thermo Scientific): Peptides of 3 or $4 \mu$ sample solution were loaded with $0.07 \%$ TFA on an Acclaim ${ }^{\circledR}$ PepMap 100 pre-column $(100 \mu \mathrm{m}$ x $2 \mathrm{~cm}, \mathrm{C} 18,3 \mu \mathrm{m}, 100 \AA$, Thermo Scientific $)$ at a flow rate of $20 \mu \mathrm{l} / \mathrm{min}$ for $3 \mathrm{~min}$. Analytical peptide separation by reverse phase chromatography was performed at a flow rate of $300 \mathrm{nl} / \mathrm{min}$ on an Acclaim ${ }^{\circledR}$ PepMap RSLC column $(75 \mu \mathrm{m}$ x $50 \mathrm{~cm}, \mathrm{C} 18$, $3 \mu \mathrm{m}, 100 \AA$, Thermo Scientific). Peptides were separated by a gradient from $96 \%$ solvent A $(0.1 \%$ formic acid) and $4 \%$ solvent $B$ ( $80 \%$ acetonitrile, $0.1 \%$ formic acid) to $45 \%$ solvent $B$ in 82 min followed by a gradient to $90 \%$ B in 12 min and a constant flow of $90 \%$ B for 3 min (Optima ${ }^{\circledR}$ LC/MS solvents and acids were purchased from Fisher Chemical).

Nano ESI mass spectrometry - Q Exactive HF (Thermo Scientific): Chromatographically eluting peptides were on-line ionized by nano-electrospray (nESI) using the Nanospray Flex Ion Source (Thermo Scientific) at $1.5 \mathrm{kV}$ (liquid junction) and continuously transferred into the mass spectrometer. Full scans within the mass range of 300-1,800 m/z were taken from the Orbitrap-FT analyzer at a resolution of 60,000 with parallel data-dependent top 10 MS2-fragmentation (HCD). The resolution of dd-MS2 scans was 15,000. For tSIM analyses, the loop count equaled the number of $\mathrm{m} / \mathrm{z}$ values on the inclusion list. LCMS method programming and data acquisition were performed with the software XCalibur 4.0 (Thermo Scientific).

\subsection{Whole-genome sequencing approaches}

\subsubsection{RNAseq}

For RNAseq, strains were grown in YNB supplemented with $1 \%$ glucose and $0.2 \%$ ammonium sulfate (YNBG) overnight to an $\mathrm{OD}_{600}$ of 0.25 and shifted to YNB supplemented with $1 \%$ arabinose and $0.2 \%$

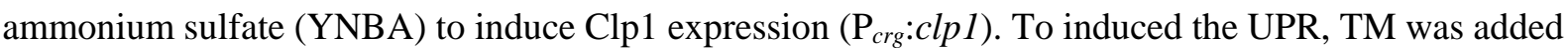


to a final concentration of $5 \mu \mathrm{g} / \mathrm{ml}$ and cells were further incubated for 4 hours at $28^{\circ} \mathrm{C}$. Cells were harvested and quick-frozen in liquid nitrogen. RNA extraction followed the procedure as described in chapter 5.3.2.

$5 \mu \mathrm{g}$ of total RNA was used to enrich mRNA using the NEB Next Poly(A) mRNA Magnetic Isolation Module (NEB) according to the manufacturer's instructions. Strand-specific cDNA libraries were constructed with the NEBNext Ultra directional RNA library preparation kit for Illumina (NEB). To assess the quality and size of the libraries samples were run on an Agilent Bioanalyzer 2100 using an Agilent High Sensitivity DNA Kit as recommended by the manufacturer (Agilent Technologies). The concentration of the libraries was determined using the Qubit ${ }^{\circledR}$ dsDNA HS Assay Kit as recommended by the manufacturer (Life Technologies). Sequencing was performed using the HiSeq4000 instrument (Illumina Inc) and the HiSeq 3000/4000 SR Cluster Kit for cluster generation and the HiSeq 3000/4000 SBS Kit (50 cycles) for sequencing in the single-end mode, running 1 x 50 cycles. A minimum of 15 Million raw reads was generated for individual samples.

Raw RNAseq reads were aligned to the Ustilago maydis genome from Ensembl Genomes 33 (Kersey et al., 2018) using STAR version 2.4.1 (Dobin et al., 2013). Read counts and RPM (reads per million) were calculated using custom Python scripts. Differential expression was assessed with DESeq2 (Love et al., 2014) at an FDR threshold of 0.05 and a log2 fold change threshold of 1 or 2 . RNAseq data were deposited at EBI ArrayExpress (https://www.ebi.ac.uk/arrayexpress/) under accession E-MTAB-7463.

\subsubsection{Chromatin immunoprecipitation sequencing (ChIPseq)}

ChIP analysis was done essentially as described before Hampel et al. (2016), with the modification that Chromatin was sheared in a Covaris S200 set to yield a DNA average size of approximately 100-300 bp. DNA was recovered by column purification (QIAquick PCR Purification Kit, Qiagen) and subjected to library preparation.

For ChIPseq experiments, the libraries were prepared from 1 ng of enriched DNA or input DNA using the NEBNext Ultra II DNA Library Prep with Beads as recommended by the manufacturer (New England BioLabs). To assess the quality and size of the libraries, samples were run on an Agilent Bioanalyzer 2100 using an Agilent High Sensitivity DNA Kit as recommended by the manufacturer (Agilent Technologies). The concentration of the libraries was determined using the Qubit ${ }^{\circledR} \mathrm{dsDNA} H S$ Assay Kit as recommended by the manufacturer (Life Technologies $\mathrm{GmbH}$ ). Libraries were sequenced on a HiSeq4000 instrument (Illumina Inc) using the HiSeq 3000/4000 SR Cluster Kit for cluster generation and the HiSeq 3000/4000 SBS Kit (50 cycles) for sequencing in the single-end mode, running 1x 50 cycles. A minimum of 40 Million raw reads was generated for the ChIPseq experiments. 
Raw ChIPseq reads were aligned using Bowtie2 version 2.0.0-beta7 (Langmead and Salzberg, 2012) to the Ustilago maydis genome from Ensembl Genomes 33 (Kersey et al., 2018). Peak calling was performed using PeakZilla, GitHub commit version 7167f084e024676bcb34e5b5c3e1281910423c25 (Bardet et al., 2013). ChIPseq data was deposited at EBI ArrayExpress (https://www.ebi.ac.uk/arrayexpress/) under accession E-MTAB-7460. Peak calling was performed individually for both biological replicates. Only peaks identified in both replicates were used for further analyses. Assignment of peaks to genes in case of divergent promoters was based on the relative distance to the translational start site (tss) and on gene expression after TM treatment. Peak scores were accumulated to promoter scores if more than one peak was identified in the promoter of a single gene and at least one peak score was above 40 . Promoter scores were filtered by a cut-off of 100 . Promoters harboring more than four peaks could never be assigned to differentially expressed genes and were thus discarded from further analysis. Normalized bigWig files were generated from BAM files derived from both replicates and visualized using the Integrative Genomics Viewer (IGV) (Robinson et al., 2017). For identification of possible binding motifs of Cib1, sequences of assigned ChIP peaks derived from the UPR core gene set were subjected to the MEME (Multiple EM for Motif Elicitation)-ChIP analysis (Machanick and Bailey, 2011).

\subsection{Bioinformatic analyses}

\subsubsection{Sequencing of DNA and plasmids used for cloning}

Sequencing of PCR products or plasmids were performed by the companies GATC Biotech AG (Konstanz) and Microsynth Seqlab (Göttingen).

\subsubsection{Sequence and structural analysis}

The following applications were used for bioinformatic analyses of DNA and protein sequences and larger data sets (RNAseq/ChIPseq/LC-MS data):

\section{ApE (A plasmid Editor) by M. Wayne Davis}

ApE was used for sequence analysis, in silico cloning and editing of plasmids and genomic sequences, primer design and multiple alignments of DNA.

mFold (Zuker, 2003)

mFold was used for the prediction of the secondary structure of qPCR amplicons (http://www.bioinfo.rpi.edu/applications/mfold/).

\section{UniProt (Universal Protein Resource)}


UniProt was used for downloading protein sequences (FASTA format) of different organisms (http://www.uniprot.org).

\section{BLASTp Basic Local Alignment Search Tool (Altschul et al., 1990; Boratyn et al., 2012)}

BLASTp was used to identify similar protein sequences to a query protein sequence as well as domains in query protein sequences (https://blast.ncbi.nlm.nih.gov/Blast.cgi?PROGRAM=blastp)

\section{FunCat}

UPR core genes were further analyzed using the Functional Catalogue annotation (FunCat) of the MIPS U. maydis database (http://mips.gsf.de/funcatDB/).

\section{Phobius (Käll et al., 2007)}

The Phobius web server was used to identify possible transmembrane domains in a query protein sequence (http://phobius.binf.ku.dk/index.html).

\section{MaxQuant (Tyanova et al., 2015) and Perseus (Tyanova et al., 2016)}

LC-MS data analysis was performed with MaxQuant (https://maxquant.org) 1.6.0.16 (parameter file in Appendix File 2) using the label-free quantification method. For statistical analysis of the MaxQuant output, the Perseus (1.6.2.3) framework was used. The heat map of potential Spp1 interaction partners (Fig 3.43) was generated in Perseus.

\section{ClustVis Web Tool (Metsalu and Vilo, 2015)}

The ClustVis Web Tool was used to generate a heat map of the identified UPR core genes (Fig 3.10). Hierarchical clustering was performed using Euclidean distance and complete linkage for genes. 


\section{Literature}

Aichinger, C., Hansson, K., Eichhorn, H., Lessing, F., Mannhaupt, G., Mewes, W. Kahmann, R. (2003) Identification of plant-regulated genes in Ustilago maydis by enhancer-trapping mutagenesis. Molecular genetics and genomics, 270, 303-314.

Altschul, S. F., Gish, W., Miller, W., Myers, E. W. Lipman, D. J. (1990) Basic local alignment search tool. Journal of molecular biology, 215, 403-410.

Arvas, M., Pakula, T., Lanthaler, K., Saloheimo, M., Valkonen, M., Suortti, T., Robson, G. Penttilä, M. (2006) Common features and interesting differences in transcriptional responses to secretion stress in the fungi Trichoderma reesei and Saccharomyces cerevisiae. BMC genomics, 7, 32.

Ausubel, F. M., R. Brent, R.E. Kingston, D.D. Moore, J.G. Seidman, J.A. Smith K. Struhl (1987) Current Protocols in Molecular Biology.

Avci, D., Fuchs, S., Schrul, B., Fukumori, A., Breker, M., Frumkin, I., Chen, C.-Y., Biniossek, M. L., Kremmer, E., Schilling, O., Steiner, H., Schuldiner, M. Lemberg, M. K. (2014) The yeast ER-intramembrane protease Ypf1 refines nutrient sensing by regulating transporter abundance. Molecular cell, 56, 630-640.

Baghirova, S., Hughes, B. G., Hendzel, M. J. Schulz, R. (2015) Sequential fractionation and isolation of subcellular proteins from tissue or cultured cells. MethodsX, 2, 440-445.

Bailey, T. L. \& Gribskov, M. (1998) Methods and statistics for combining motif match scores. Journal of computational biology: a journal of computational molecular cell biology, 5, 211-221.

Baldwin, M., Russo, C., Li, X. Chishti, A. H. (2014) Plasmodium falciparum signal peptide peptidase cleaves malaria heat shock protein 101 (HSP101). Implications for gametocytogenesis. Biochemical and biophysical research communications, 450, 1427-1432.

Banuett, F. (1995) Genetics of Ustilago maydis, a fungal pathogen that induces tumors in maize. Annual review of genetics, 29, 179-208.

Banuett, F. \& Herskowitz, I. (1988) Ustilago maydis, smut of maize. Genetics of Plant Pathogenic Fungi: Advances in Plant Pathology, 427-455.

Banuett, F. \& Herskowitz, I. (1996) Discrete developmental stages during teliospore formation in the corn smut fungus, Ustilago maydis. Development (Cambridge, England), 122, 2965-2976.

Bardet, A. F., Steinmann, J., Bafna, S., Knoblich, J. A., Zeitlinger, J. Stark, A. (2013) Identification of transcription factor binding sites from ChIP-seq data at high resolution. Bioinformatics, 29, 27052713. 
Bat-Ochir, C., Kwak, J.-Y., Koh, S.-K., Jeon, M.-H., Chung, D., Lee, Y.-W. Chae, S.-K. (2016) The signal peptide peptidase SppA is involved in sterol regulatory element-binding protein cleavage and hypoxia adaptation in Aspergillus nidulans. Molecular microbiology, 100, 635-655.

Becht, P., König, J. Feldbrügge, M. (2006) The RNA-binding protein Rrm4 is essential for polarity in Ustilago maydis and shuttles along microtubules. Journal of cell science, 119, 4964-4973.

Blom, N., Gammeltoft, S. Brunak, S. (1999) Sequence and structure-based prediction of eukaryotic protein phosphorylation sites. Journal of molecular biology, 294, 1351-1362.

Bölker, M., Genin, S., Lehmler, C. Kahmann, R. (1995) Genetic regulation of mating and dimorphism in Ustilago maydis. Canadian Journal of Botany, 73, 320-325.

Bölker, M., Urban, M. Kahmann, R. (1992) The a mating type locus of $U$. maydis specifies cell signaling components. Cell, 68, 441-450.

Boller, T. \& He, S. Y. (2009) Innate immunity in plants: an arms race between pattern recognition receptors in plants and effectors in microbial pathogens. Science, 324, 742-744.

Bolwell, G. P. \& Wojtaszek, P. (1997) Mechanisms for the generation of reactive oxygen species in plant defence - a broad perspective. Physiological and Molecular Plant Pathology, 51, 347-366.

Boname, J. M., Bloor, S., Wandel, M. P., Nathan, J. A., Antrobus, R., Dingwell, K. S., Thurston, T. L., Smith, D. L., Smith, J. C., Randow, F. Lehner, P. J. (2014) Cleavage by signal peptide peptidase is required for the degradation of selected tail-anchored proteins. The Journal of cell biology, 205, 847-862.

Boratyn, G. M., Schäffer, A. A., Agarwala, R., Altschul, S. F., Lipman, D. J. Madden, T. L. (2012) Domain enhanced lookup time accelerated BLAST. Biology direct, 7, 12.

Bottin, A., Kämper, J. Kahmann, R. (1996) Isolation of a carbon source-regulated gene from Ustilago maydis. Molecular \& General Genetics, 253, 342-352.

Brachmann, A. (2001) Die frühe Infektionsphase von Ustilago maydis. Dissertation. Fakultät für Biologie. Ludwig-Maximilians-Universität München.

Brachmann, A., König, J., Julius, C. Feldbrügge, M. (2004) A reverse genetic approach for generating gene replacement mutants in Ustilago maydis. Molecular genetics and genomics, 272, 216-226.

Brachmann, A., Schirawski, J., Müller, P. Kahmann, R. (2003) An unusual MAP kinase is required for efficient penetration of the plant surface by Ustilago maydis. The EMBO journal, 22, 2199-2210.

Brachmann, A., Weinzierl, G., Kämper, J. Kahmann, R. (2001) Identification of genes in the bW/bE regulatory cascade in Ustilago maydis. Molecular microbiology, 42, 1047-1063. 
Brefort, T., Tanaka, S., Neidig, N., Doehlemann, G., Vincon, V. Kahmann, R. (2014) Characterization of the largest effector gene cluster of Ustilago maydis. PLoS pathogens, 10, e1003866.

Broomfield, P. L. \& Hargreaves, J. A. (1992) A single amino-acid change in the iron-sulphur protein subunit of succinate dehydrogenase confers resistance to carboxin in Ustilago maydis. Current genetics, 22, 117-121.

Carvalho, N. D., Jørgensen, T. R., Arentshorst, M., Nitsche, B. M., van den Hondel, C. A., Archer, D. B. Ram, A. F. (2012) Genome-wide expression analysis upon constitutive activation of the HacA bZIP transcription factor in Aspergillus niger reveals a coordinated cellular response to counteract ER stress. BMC genomics, 13, 350.

Casadaban, M. J. \& Cohen, S. N. (1980) Analysis of gene control signals by DNA fusion and cloning in Escherichia coli. Journal of molecular biology, 138, 179-207.

Chen, C.-Y., Malchus, N. S., Hehn, B., Stelzer, W., Avci, D., Langosch, D. Lemberg, M. K. (2014) Signal peptide peptidase functions in ERAD to cleave the unfolded protein response regulator XBP1u. The EMBO journal, 33, 2492-2506.

Cheon, S. A., Jung, K.-W., Bahn, Y.-S. Kang, H. A. (2014) The unfolded protein response (UPR) pathway in Cryptococcus. Virulence, 5, 341-350.

Cheon, S. A., Jung, K.-W., Chen, Y.-L., Heitman, J., Bahn, Y.-S. Kang, H. A. (2011) Unique evolution of the UPR pathway with a novel bZIP transcription factor, Hxl1, for controlling pathogenicity of Cryptococcus neoformans. PLoS pathogens, 7, e1002177.

Chi, Y., Huddleston, M. J., Zhang, X., Young, R. A., Annan, R. S., Carr, S. A. Deshaies, R. J. (2001) Negative regulation of Gcn4 and Msn2 transcription factors by Srb10 cyclin-dependent kinase. Genes \& development, 15, 1078-1092.

Chojnacki, S., Cowley, A., Lee, J., Foix, A. Lopez, R. (2017) Programmatic access to bioinformatics tools from EMBL-EBI update: 2017. Nucleic acids research, 45, W550-3.

Christensen, J. J. (1963) Corn smut caused by Ustilago maydis. American Phytopathological Society, $1-40$.

Cohen, N., Breker, M., Bakunts, A., Pesek, K., Chas, A., Argemí, J. et al. (2017) Iron affects Ire1 clustering propensity and the amplitude of endoplasmic reticulum stress signaling. Journal of cell science, 130, 3222-3233.

Coulthard, L. R., White, D. E., Jones, D. L., McDermott, M. F. Burchill, S. A. (2009) p38(MAPK): stress responses from molecular mechanisms to therapeutics. Trends in molecular medicine, 15, 369379. 
Cox, J. S. \& Walter, P. (1996) A novel mechanism for regulating activity of a transcription factor that controls the unfolded protein response. Cell, 87, 391-404.

Davies, J. R., Osmani, A. H., Souza, C. P. C. de, Bachewich, C. Osmani, S. A. (2004) Potential link between the NIMA mitotic kinase and nuclear membrane fission during mitotic exit in Aspergillus nidulans. Eukaryotic Cell, 3, 1433-1444.

Dillin, A. (2014) Profile of Kazutoshi Mori and Peter Walter, 2014 Lasker Basic Medical Research awardees: The unfolded protein response. Proceedings of the National Academy of Sciences of the United States of America, 111, 17696-17697.

Djamei, A., Schipper, K., Rabe, F., Ghosh, A., Vincon, V., Kahnt, J. et al. (2011) Metabolic priming by a secreted fungal effector. Nature, 478, 395-398.

Dobin, A., Davis, C. A., Schlesinger, F., Drenkow, J., Zaleski, C., Jha, S., Batut, P., Chaisson, M. Gingeras, T. R. (2013) STAR: ultrafast universal RNA-seq aligner. Bioinformatics (Oxford, England), 29, 15-21.

Doebley, J. (1992) Mapping the genes that made maize. Trends in genetics, 8, 302-307.

Doehlemann, G., Reissmann, S., Assmann, D., Fleckenstein, M. Kahmann, R. (2011) Two linked genes encoding a secreted effector and a membrane protein are essential for Ustilago maydis-induced tumour formation. Molecular microbiology, 81, 751-766.

Doehlemann, G., van der Linde, K., Assmann, D., Schwammbach, D., Hof, A., Mohanty, A., Jackson, D. Kahmann, R. (2009) Pep1, a secreted effector protein of Ustilago maydis, is required for successful invasion of plant cells. PLoS pathogens, 5, e1000290.

Durfee, T., Nelson, R., Baldwin, S., Plunkett, G., Burland, V., Mau, B., Petrosino, J. F., Qin, X., Muzny, D. M., Ayele, M., Gibbs, R. A., Csörgo, B., Pósfai, G., Weinstock, G. M. Blattner, F. R. (2008) The complete genome sequence of Escherichia coli DH10B: insights into the biology of a laboratory workhorse. Journal of Bacteriology, 190, 2597-2606.

Fang, H., Mullins, C. Green, N. (1997) In Addition to SEC11, a Newly Identified Gene, SPC3, Is Essential for Signal Peptidase Activity in the Yeast Endoplasmic Reticulum. The Journal of biological chemistry, 272, 13152-13158.

Feldheim, D., Yoshimura, K., Admon, A. Schekman, R. (1993) Structural and functional characterization of Sec66p, a new subunit of the polypeptide translocation apparatus in the yeast endoplasmic reticulum. Molecular biology of the cell, 4, 931-939.

Fernández-Alvarez, A., Elías-Villalobos, A. Ibeas, J. I. (2009) The O-mannosyltransferase PMT4 is essential for normal appressorium formation and penetration in Ustilago maydis. The Plant cell, 21, 3397-3412. 
Fernández-Álvarez, A., Marín-Menguiano, M., Lanver, D., Jiménez-Martín, A., Elías-Villalobos, A., Pérez-Pulido, A. J., Kahmann, R. Ibeas, J. I. (2012) Identification of O-mannosylated virulence factors in Ustilago maydis. PLoS pathogens, 8, e1002563.

Filipe, A. \& McLauchlan, J. (2015) Hepatitis C virus and lipid droplets: finding a niche. Trends in molecular medicine, 21, 34-42.

Filtz, T. M., Vogel, W. K. Leid, M. (2014) Regulation of transcription factor activity by interconnected post-translational modifications. Trends in pharmacological sciences, 35, 76-85.

Flajnik, M. F. \& Kasahara, M. (2001) Comparative Genomics of the MHC. Immunity, 15, 351-362.

Fluhrer, R., Steiner, H. Haass, C. (2009) Intramembrane Proteolysis by Signal Peptide Peptidases: A Comparative Discussion of GXGD-type Aspartyl Proteases. The Journal of biological chemistry, 284, 13975-13979.

Fordyce, P. M., Pincus, D., Kimmig, P., Nelson, C. S., El-Samad, H., Walter, P. DeRisi, J. L. (2012) Basic leucine zipper transcription factor Hac1 binds DNA in two distinct modes as revealed by microfluidic analyses. Proceedings of the National Academy of Sciences of the United States of America, 109, E3084-93.

Fox, R. M., Hanlon, C. D. Andrew, D. J. (2010) The CrebA/Creb3-like transcription factors are major and direct regulators of secretory capacity. The Journal of cell biology, 191, 479-492.

Frand, A. R. \& Kaiser, C. A. (1998) The ERO1 Gene of Yeast Is Required for Oxidation of Protein Dithiols in the Endoplasmic Reticulum. Molecular cell, 1, 161-170.

Fuchs, S. Y., Dolan, L., Davis, R. J. Ronai, Z. (1996) Phosphorylation-dependent targeting of c-Jun ubiquitination by Jun N-kinase. Oncogene, 13, 1531-1535.

Gan, L., Zheng, W., Chabot, J.-G., Unterman, T. G. Quirion, R. (2005) Nuclear/cytoplasmic shuttling of the transcription factor FoxO1 is regulated by neurotrophic factors. Journal of neurochemistry, 93, 1209-1219.

Gardner, B. M. \& Walter, P. (2011) Unfolded proteins are Ire1-activating ligands that directly induce the unfolded protein response. Science, 333, 1891-1894.

Gillissen, B., Bergemann, J., Sandmann, C., Schroeer, B., Bölker, M. Kahmann, R. (1992) A twocomponent regulatory system for self/non-self recognition in Ustilago maydis. Cell, 68, 647-657.

Glass, N. L. \& Dementhon, K. (2006) Non-self recognition and programmed cell death in filamentous fungi. Current opinion in microbiology, 9, 553-558.

Glass, N. L. \& Kaneko, I. (2003) Fatal Attraction: Nonself Recognition and Heterokaryon Incompatibility in Filamentous Fungi. Eukaryotic Cell, 2, 1-8. 
Glazebrook, J. (2005) Contrasting mechanisms of defense against biotrophic and necrotrophic pathogens. Annual review of phytopathology, 43, 205-227.

Glazier, V. E., Kaur, J. N., Brown, N. T., Rivera, A. A. Panepinto, J. C. (2015) Puf4 regulates both splicing and decay of HXL1 mRNA encoding the unfolded protein response transcription factor in Cryptococcus neoformans. Eukaryotic Cell, 14, 385-395.

Golde, T. E., Wolfe, M. S. Greenbaum, D. C. (2009) Signal peptide peptidases: a family of intramembrane-cleaving proteases that cleave type 2 transmembrane proteins. Seminars in cell \& developmental biology, 20, 225-230.

Gonzalez, T. N., Sidrauski, C., Dörfler, S. Walter, P. (1999) Mechanism of non-spliceosomal mRNA splicing in the unfolded protein response pathway. The EMBO journal, 18, 3119-3132.

Grigorenko, A. P., Moliaka, Y. K., Korovaitseva, G. I. Rogaev, E. I. (2002) Novel class of polytopic proteins with domains associated with putative protease activity. Biochemistry. Biokhimiia, 67, 826835.

Guillemette, T., Calmes, B. Simoneau, P. (2013) Impact of the UPR on the virulence of the plant fungal pathogen A. brassicicola. Virulence, 5, 357-364.

Hach, C. (2018) Functional characterization of Clp1 modulated UPR genes in Ustilago maydis. Master thesis. Faculty of Biology and Psychology. Georg-August-University Göttingen.

Halbleib, K., Pesek, K., Covino, R., Hofbauer, H. F., Wunnicke, D., Hänelt, I., Hummer, G. Ernst, R. (2017) Activation of the Unfolded Protein Response by Lipid Bilayer Stress. Molecular cell, 67, 673-684.e8.

Hampel, M. (2016) Analysis of the UPR mediated stress response and its function during the biotrophic development of Ustilago maydis. Dissertation. Faculty of Biology and Psychology. Georg-AugustUniversity Göttingen.

Hampel, M., Jakobi, M., Schmitz, L., Meyer, U., Finkernagel, F., Doehlemann, G. Heimel, K. (2016) Unfolded Protein Response (UPR) Regulator Cib1 Controls Expression of Genes Encoding Secreted Virulence Factors in Ustilago maydis. PloS one, 11, e0153861.

Hanahan, D., Jessee, J. Bloom, F. R. (1991) Plasmid transformation of Escherichia coli and other bacteria. Methods in enzymology, 204, 63-113.

Harbut, M. B., Patel, B. A., Yeung, B. K. S., McNamara, C. W., Bright, A. T., Ballard, J., Supek, F., Golde, T. E., Winzeler, E. A., Diagana, T. T. Greenbaum, D. C. (2012) Targeting the ERAD pathway via inhibition of signal peptide peptidase for antiparasitic therapeutic design. Proceedings of the National Academy of Sciences of the United States of America, 109, 21486-21491. 
Hartmann, H. A., Kahmann, R. Bölker, M. (1996) The pheromone response factor coordinates filamentous growth and pathogenicity in Ustilago maydis. The EMBO journal, 15, 1632-1641.

Heimel, K. (2010) Regulationsmechanismen der biotrophen Entwicklung von Ustilago maydis. Dissertation. Fachbereich Biologie. Philipps-Universität Marburg.

Heimel, K., Freitag, J., Hampel, M., Ast, J., Bölker, M. Kämper, J. (2013) Crosstalk between the unfolded protein response and pathways that regulate pathogenic development in Ustilago maydis. The Plant cell, 25, 4262-4277.

Heimel, K., Scherer, M., Schuler, D. Kämper, J. (2010a) The Ustilago maydis Clp1 protein orchestrates pheromone and $b$-dependent signaling pathways to coordinate the cell cycle and pathogenic development. The Plant cell, 22, 2908-2922.

Heimel, K., Scherer, M., Vranes, M., Wahl, R., Pothiratana, C., Schuler, D., Vincon, V., Finkernagel, F., Flor-Parra, I. Kämper, J. (2010b) The transcription factor Rbf1 is the master regulator for $b$-mating type controlled pathogenic development in Ustilago maydis. PLoS pathogens, 6, e1001035.

Hemetsberger, C., Herrberger, C., Zechmann, B., Hillmer, M. Doehlemann, G. (2012) The Ustilago maydis effector Pep1 suppresses plant immunity by inhibition of host peroxidase activity. PLoS pathogens, 8, e1002684.

Hetz, C. (2012) The unfolded protein response: controlling cell fate decisions under ER stress and beyond. Nature reviews. Molecular cell biology, 13, 89-102.

Hoffman, C. S. \& Winston, F. (1987) A ten-minute DNA preparation from yeast efficiently releases autonomous plasmids for transformation of Escherichia coli. Gene, 57, 267-272.

Holliday, R. Ustilago maydis. Bacteria, Bacteriophages, and Fungi, 1974, 575-595.

Holliday, R. (1964) The Induction of Mitotic Recombination by Mitomycin C in Ustilago and Saccharomyces. Genetics, 50, 323-335.

Hollien, J., Lin, J. H., Li, H., Stevens, N., Walter, P. Weissman, J. S. (2009) Regulated Ire1dependent decay of messenger RNAs in mammalian cells. The Journal of cell biology, 186, 323331.

Hsu, F.-F., Chou, Y.-T., Chiang, M.-T., Li, F.-A., Yeh, C.-T., Lee, W.-H. Chau, L.-Y. (2018) Signal peptide peptidase promotes tumor progression via facilitating FKBP8 degradation. Oncogene.

Hu, H., Gao, J., He, J., Yu, B., Zheng, P., Huang, Z., Mao, X., Yu, J., Han, G. Chen, D. (2013) Codon optimization significantly improves the expression level of a keratinase gene in Pichia pastoris. PloS one, 8, e58393. 
Hughes, A. L., Powell, D. W., Bard, M., Eckstein, J., Barbuch, R., Link, A. J. Espenshade, P. J. (2007) Dap1/PGRMC1 binds and regulates cytochrome P450 enzymes. Cell metabolism, 5, 143149.

Innis, M. A. (1990) PCR protocols. A guide to methods and applications. Academic Press

Irieda, H., Inoue, Y., Mori, M., Yamada, K., Oshikawa, Y., Saitoh, H., Uemura, A., Terauchi, R., Kitakura, S., Kosaka, A., Singkaravanit-Ogawa, S. Takano, Y. (2019) Conserved fungal effector suppresses PAMP-triggered immunity by targeting plant immune kinases. Proceedings of the National Academy of Sciences of the United States of America, 116, 496-505.

Irniger, S. \& Braus, G. H. (2003) Controlling transcription by destruction: the regulation of yeast Gcn4p stability. Current genetics, 44, 8-18.

Ivanova, A. V., Ivanov, S. V. Lerman, M. L. (2005) Association, mutual stabilization, and transcriptional activity of the STRA13 and MSP58 proteins. CMLS, Cell. Mol. Life Sci., 62, 471484.

Janczak, M., Bukowski, M., Górecki, A., Dubin, G., Dubin, A. Wladyka, B. (2015) A systematic investigation of the stability of green fluorescent protein fusion proteins. Acta biochimica Polonica, 62, 407-411.

Jantz, D. \& Berg, J. M. (2004) Reduction in DNA-binding affinity of Cys2His2 zinc finger proteins by linker phosphorylation. Proceedings of the National Academy of Sciences of the United States of America, 101, 7589-7593.

Jolma, A., Yan, J., Whitington, T., Toivonen, J., Nitta, K. R., Rastas, P. et al. (2013) DNA-binding specificities of human transcription factors. Cell, 152, 327-339.

Jonge, R. de, Bolton, M. D. Thomma, B. P. H. J. (2011) How filamentous pathogens co-opt plants: the ins and outs of fungal effectors. Current opinion in plant biology, 14, 400-406.

Joubert, A., Simoneau, P., Campion, C., Bataillé-Simoneau, N., Iacomi-Vasilescu, B., Poupard, P., François, J. M., Georgeault, S., Sellier, E. Guillemette, T. (2011) Impact of the unfolded protein response on the pathogenicity of the necrotrophic fungus Alternaria brassicicola. Molecular microbiology, 79, 1305-1324.

Jung, K.-W., Kang, H. A. Bahn, Y.-S. (2013) Essential roles of the Kar2/BiP molecular chaperone downstream of the UPR pathway in Cryptococcus neoformans. PloS one, 8, e58956.

Kaffarnik, F., Müller, P., Leibundgut, M., Kahmann, R. Feldbrügge, M. (2003) PKA and MAPK phosphorylation of Prf1 allows promoter discrimination in Ustilago maydis. The EMBO journal, 22, 5817-5826. 
Kalies, K.-U., Rapoport, T. A. Hartmann, E. (1998) The $\beta$ Subunit of the Sec61 Complex Facilitates Cotranslational Protein Transport and Interacts with the Signal Peptidase during Translocation. The Journal of cell biology, 141, 887-894.

Käll, L., Krogh, A. Sonnhammer, E. L. L. (2004) A combined transmembrane topology and signal peptide prediction method. Journal of molecular biology, 338, 1027-1036.

Käll, L., Krogh, A. Sonnhammer, E. L. L. (2007) Advantages of combined transmembrane topology and signal peptide prediction-the Phobius web server. Nucleic acids research, 35, W429-32.

Kämper, J. (2004) A PCR-based system for highly efficient generation of gene replacement mutants in Ustilago maydis. Molecular genetics and genomics, 271, 103-110.

Kämper, J., Kahmann, R., Bölker, M., Ma, L.-J., Brefort, T., Saville, B. J. et al. (2006) Insights from the genome of the biotrophic fungal plant pathogen Ustilago maydis. Nature, 444, 97-101.

Kämper, J., Reichmann, M., Romeis, T., Bölker, M. Kahmann, R. (1995) Multiallelic recognition: Nonself-dependent dimerization of the bE and bW homeodomain proteins in Ustilago maydis. Cell, 81, 73-83.

Kaneko, I., Dementhon, K., Xiang, Q. Glass, N. L. (2006) Nonallelic interactions between het-c and a polymorphic locus, pin-c, are essential for nonself recognition and programmed cell death in Neurospora crassa. Genetics, 172, 1545-1555.

Kanemoto, S., Kondo, S., Ogata, M., Murakami, T., Urano, F. Imaizumi, K. (2005) XBP1 activates the transcription of its target genes via an ACGT core sequence under ER stress. Biochemical and biophysical research communications, 331, 1146-1153.

Karagöz, G. E., Acosta-Alvear, D. Walter, P. (2019) The Unfolded Protein Response: Detecting and Responding to Fluctuations in the Protein-Folding Capacity of the Endoplasmic Reticulum. Cold Spring Harbor perspectives in biology.

Kawano, T. (2003) Roles of the reactive oxygen species-generating peroxidase reactions in plant defense and growth induction. Plant cell reports, 21, 829-837.

Kelleher, D. J. \& Gilmore, R. (1994) The Saccharomyces cerevisiae oligosaccharyltransferase is a protein complex composed of Wbp1p, Swp1p, and four additional polypeptides. The Journal of biological chemistry, 269, 12908-12917.

Kersey, P. J., Allen, J. E., Allot, A., Barba, M., Boddu, S., Bolt, B. J. et al. (2018) Ensembl Genomes 2018: an integrated omics infrastructure for non-vertebrate species. Nucleic acids research, 46, D802-D808. 
Kornitzer, D., Raboy, B., Kulka, R. G. Fink, G. R. (1994) Regulated degradation of the transcription factor Gcn4. The EMBO journal, 13, 6021-6030.

Krishnan, K., Feng, X., Powers-Fletcher, M. V., Bick, G., Richie, D. L., Woollett, L. A. Askew, D. S. (2013) Effects of a defective endoplasmic reticulum-associated degradation pathway on the stress response, virulence, and antifungal drug susceptibility of the mold pathogen Aspergillus fumigatus. Eukaryotic Cell, 12, 512-519.

La Rosa, J. M. de, González, J. M., Gutiérrez, F., Ruíz, T. Rodríguez, L. (2004) Characterization of Candida albicans orthologue of the Saccharomyces cerevisiae signal-peptidase-subunit encoding gene SPC3. Yeast, 21, 883-894.

Lachowiec, J., Queitsch, C. Kliebenstein, D. J. (2016) Molecular mechanisms governing differential robustness of development and environmental responses in plants. Annals of botany, 117, 795-809.

Laemmli, U. K. (1970) Cleavage of Structural Proteins during the Assembly of the Head of Bacteriophage T4. Nature, 227, 680.

Langmead, B. \& Salzberg, S. L. (2012) Fast gapped-read alignment with Bowtie 2. Nature Methods, 9, 357.

Lanver, D., Berndt, P., Tollot, M., Naik, V., Vranes, M., Warmann, T., Münch, K., Rössel, N. Kahmann, R. (2014) Plant surface cues prime Ustilago maydis for biotrophic development. PLoS pathogens, 10, e1004272.

Lanver, D., Müller, A. N., Happel, P., Schweizer, G., Haas, F. B., Franitza, M., Pellegrin, C., Reissmann, S., Altmüller, J., Rensing, S. A. Kahmann, R. (2018) The Biotrophic Development of Ustilago maydis Studied by RNA-Seq Analysis. The Plant cell, 30, 300-323.

Lanver, D., Tollot, M., Schweizer, G., Lo Presti, L., Reissmann, S., Ma, L.-S., Schuster, M., Tanaka, S., Liang, L., Ludwig, N. Kahmann, R. (2017) Ustilago maydis effectors and their impact on virulence. Nature Reviews Microbiology, 15, 409 EP -.

Lee, S.-O., Cho, K., Cho, S., Kim, I., Oh, C. Ahn, K. (2010) Protein disulphide isomerase is required for signal peptide peptidase-mediated protein degradation. The EMBO journal, 29, 363-375.

Lemberg, M. K., Bland, F. A., Weihofen, A., Braud, V. M. Martoglio, B. (2001) Intramembrane Proteolysis of Signal Peptides: An Essential Step in the Generation of HLA-E Epitopes. The Journal of Immunology, 167, 6441-6446.

Lemberg, M. K. \& Martoglio, B. (2004) On the mechanism of SPP-catalysed intramembrane proteolysis; conformational control of peptide bond hydrolysis in the plane of the membrane. FEBS Letters, 564, 213-218. 
Li, Z., Srivastava, R., Tang, J., Zheng, Z. Howell, S. H. (2018) Cis-Effects Condition the Induction of a Major Unfolded Protein Response Factor, ZmbZIP60, in Response to Heat Stress in Maize. Frontiers in plant science, 9, 833.

Liu, C., Pedersen, C., Schultz-Larsen, T., Aguilar, G. B., Madriz-Ordeñana, K., Hovmøller, M. S. Thordal-Christensen, H. (2016) The stripe rust fungal effector PEC6 suppresses pattern-triggered immunity in a host species-independent manner and interacts with adenosine kinases. The New phytologist.

Lo Presti, L., Lanver, D., Schweizer, G., Tanaka, S., Liang, L., Tollot, M., Zuccaro, A., Reissmann, S. Kahmann, R. (2015) Fungal effectors and plant susceptibility. Annual review of plant biology, 66, 513-545.

Lo Presti, L., López Díaz, C., Turrà, D., Di Pietro, A., Hampel, M., Heimel, K. Kahmann, R. (2016) A conserved co-chaperone is required for virulence in fungal plant pathogens. The New phytologist, 209, 1135-1148.

Love, M. I., Huber, W. Anders, S. (2014) Moderated estimation of fold change and dispersion for RNA-seq data with DESeq2. Genome biology, 15, 550.

Lutzoni, F., Kauff, F., Cox, C. J., McLaughlin, D., Celio, G., Dentinger, B. et al. (2004) Assembling the fungal tree of life: progress, classification, and evolution of subcellular traits. American journal of botany, $91,1446-1480$.

Machanick, P. \& Bailey, T. L. (2011) MEME-ChIP: motif analysis of large DNA datasets. Bioinformatics (Oxford, England), 27, 1696-1697.

Magnani, M., Crinelli, R., Bianchi, M. Antonelli, A. (2000) The Ubiquitin-Dependent Proteolytic System and other Potential Targets for the Modulation of Nuclear Factor-kB (NF-kB). Current Drug Targets, 1, 387-399.

Marschall, R. \& Tudzynski, P. (2017) The Protein Disulfide Isomerase of Botrytis cinerea: An ER Protein Involved in Protein Folding and Redox Homeostasis Influences NADPH Oxidase Signaling Processes. Frontiers in microbiology, 8, 960.

Matsuzawa, A., Nishitoh, H., Tobiume, K., Takeda, K. Ichijo, H. (2002) Physiological roles of ASK1-mediated signal transduction in oxidative stress- and endoplasmic reticulum stress-induced apoptosis: advanced findings from ASK1 knockout mice. Antioxidants \& redox signaling, 4, 415425.

McLauchlan, J., Lemberg, M. K., Hope, G. Martoglio, B. (2002) Intramembrane proteolysis promotes trafficking of hepatitis C virus core protein to lipid droplets. The EMBO journal, 21, 39803988. 
Mestrom, L., Marsden, S. R., Dieters, M., Achterberg, P., Stolk, L., Bento, I., Hanefeld, U. Hagedoorn, P.-L. (2019) Artificial Fusion of mCherry Enhanced Solubility and Stability of Trehalose Transferase. Applied and environmental microbiology.

Metsalu, T. \& Vilo, J. (2015) ClustVis: a web tool for visualizing clustering of multivariate data using Principal Component Analysis and heatmap. Nucleic acids research, 43, W566-70.

Meyer, H. A. \& Hartmann, E. (1997) The yeast SPC22/23 homolog Spc3p is essential for signal peptidase activity. The Journal of biological chemistry, 272, 13159-13164.

Molina, L. \& Kahmann, R. (2007) An Ustilago maydis gene involved in H2O2 detoxification is required for virulence. The Plant cell, 19, 2293-2309.

Mori, K. (2009) Signalling pathways in the unfolded protein response: development from yeast to mammals. Journal of biochemistry, 146, 743-750.

Mori, K., Kawahara, T., Yoshida, H., Yanagi, H. Yura, T. (1996) Signalling from endoplasmic reticulum to nucleus: transcription factor with a basic-leucine zipper motif is required for the unfolded protein-response pathway. Genes Cells, 1, 803-817.

Müller, O., Kahmann, R., Aguilar, G., Trejo-Aguilar, B., Wu, A. Vries, R. P. de (2008) The secretome of the maize pathogen Ustilago maydis. Fungal genetics and biology, 45 Suppl 1, S63-70.

Munkvold, G. P. \& White, D. G. (2016) Compendium of Corn Diseases, Fourth Edition. The American Phytopathological Society.

Okamura, K., Kimata, Y., Higashio, H., Tsuru, A. Kohno, K. (2000) Dissociation of Kar2p/BiP from an ER sensory molecule, Ire1p, triggers the unfolded protein response in yeast. Biochemical and biophysical research communications, 279, 445-450.

Ökmen, B., Mathow, D., Hof, A., Lahrmann, U., Aßmann, D. Doehlemann, G. (2018) Mining the effector repertoire of the biotrophic fungal pathogen Ustilago hordei during host and non-host infection. Molecular plant pathology, 19, 2603-2622.

Oliveira, C. C., Querido, B., Sluijter, M., Groot, A. F. de, van der Zee, R., Rabelink, M. J. W. E., Hoeben, R. C., Ossendorp, F., van der Burg, S. H. van Hall, T. (2013) New role of signal peptide peptidase to liberate C-terminal peptides for MHC class I presentation. Journal of immunology (Baltimore, Md. : 1950), 191, 4020-4028.

Olson, G. M., Fox, D. S., Wang, P., Alspaugh, J. A. Buchanan, K. L. (2007) Role of protein Omannosyltransferase Pmt4 in the morphogenesis and virulence of Cryptococcus neoformans. Eukaryotic Cell, 6, 222-234. 
Oresic, K., Ng, C. L. Tortorella, D. (2009) TRAM1 participates in human cytomegalovirus US2- and US11-mediated dislocation of an endoplasmic reticulum membrane glycoprotein. The Journal of biological chemistry, 284, 5905-5914.

Oslowski, C. M. \& Urano, F. (2011) Measuring ER stress and the unfolded protein response using mammalian tissue culture system. Methods in enzymology, 490, 71-92.

Oyadomari, S., Koizumi, A., Takeda, K., Gotoh, T., Akira, S., Araki, E. Mori, M. (2002) Targeted disruption of the Chop gene delays endoplasmic reticulum stress-mediated diabetes. The Journal of clinical investigation, 109, 525-532.

Paetzel, M., Karla, A., Strynadka, N. C. J. Dalbey, R. E. (2002) Signal Peptidases. Chemical reviews, 102, 4549-4580.

Pal, B., Chan, N. C., Helfenbaum, L., Tan, K., Tansey, W. P. Gething, M.-J. (2007) SCFCdc4mediated degradation of the Hac1p transcription factor regulates the unfolded protein response in Saccharomyces cerevisiae. Molecular biology of the cell, 18, 426-440.

Panadero, J., Pallotti, C., Rodríguez-Vargas, S., Randez-Gil, F. Prieto, J. A. (2006) A downshift in temperature activates the high osmolarity glycerol (HOG) pathway, which determines freeze tolerance in Saccharomyces cerevisiae. The Journal of biological chemistry, 281, 4638-4645.

Park, C.-H., Chen, S., Shirsekar, G., Zhou, B., Khang, C. H., Songkumarn, P., Afzal, A. J., Ning, Y., Wang, R., Bellizzi, M., Valent, B. Wang, G.-L. (2012) The Magnaporthe oryzae effector AvrPiz-t targets the RING E3 ubiquitin ligase APIP6 to suppress pathogen-associated molecular pattern-triggered immunity in rice. The Plant cell, 24, 4748-4762.

Patel, V. J., Thalassinos, K., Slade, S. E., Connolly, J. B., Crombie, A., Murrell, J. C. Scrivens, J. H. (2009) A comparison of labeling and label-free mass spectrometry-based proteomics approaches. Journal of proteome research, 8, 3752-3759.

Perkins, D. d. (1988) Main features of vegetative incompatibility in Neurospora. Fungal Genetics Reports, 35, 44.

Petersen, T. N., Brunak, S., Heijne, G. von Nielsen, H. (2011) SignalP 4.0: discriminating signal peptides from transmembrane regions. Nature Methods, 8, 785-786.

Ponting, C. P., Hutton, M., Nyborg, A. C., Baker, M., Jansen, K. R. Golde, T. E. (2002) Identification of a novel family of presenilin homologues. Human molecular genetics, 11 9, 1037 1044.

Rabe, F., Bosch, J., Stirnberg, A., Guse, T., Bauer, L., Seitner, D. et al. (2016) A complete toolset for the study of Ustilago bromivora and Brachypodium sp. as a fungal-temperate grass pathosystem. eLife, 5. 
Rappsilber, J., Ishihama, Y. Mann, M. (2003) Stop and Go Extraction Tips for Matrix-Assisted Laser Desorption/Ionization, Nanoelectrospray, and LC/MS Sample Pretreatment in Proteomics. Anal. Chem., 75, 663-670.

Rappsilber, J., Mann, M. Ishihama, Y. (2007) Protocol for micro-purification, enrichment, prefractionation and storage of peptides for proteomics using StageTips. Nature protocols, 2, 18961906.

Reimold, A. M., Iwakoshi, N. N., Manis, J., Vallabhajosyula, P., Szomolanyi-Tsuda, E., Gravallese, E. M., Friend, D., Grusby, M. J., Alt, F. Glimcher, L. H. (2001) Plasma cell differentiation requires the transcription factor XBP-1. Nature, 412, 300-307.

Richie, D. L., Hartl, L., Aimanianda, V., Winters, M. S., Fuller, K. K., Miley, M. D., White, S., McCarthy, J. W., Latgé, J.-P., Feldmesser, M., Rhodes, J. C. Askew, D. S. (2009) A role for the unfolded protein response (UPR) in virulence and antifungal susceptibility in Aspergillus fumigatus. PLoS pathogens, 5, e1000258.

Robinson, J. T., Thorvaldsdóttir, H., Wenger, A. M., Zehir, A. Mesirov, J. P. (2017) Variant Review with the Integrative Genomics Viewer. Cancer research, 77, e31-e34.

Ruggiano, A., Foresti, O. Carvalho, P. (2014) Quality control: ER-associated degradation: protein quality control and beyond. The Journal of cell biology, 204, 869-879.

Sambrook, J., Fritsch, E. F., Maniatis, T. Cold Spring Harbor Laboratory (1989) Molecular cloning: a laboratory manual. 2nd ed.

Sambrook, J. \& Russell, D. W. (2001) Molecular cloning: A laboratory manual. 3rd ed.

Sanders, S. L., Gentzsch, M., Tanner, W. Herskowitz, I. (1999) O-Glycosylation of Axl2/Bud10p by Pmt4p Is Required for Its Stability, Localization, and Function in Daughter Cells. The Journal of cell biology, 145, 1177-1188.

Sarkar, S., Iyer, G., Wu, J. Glass, N.L. (2002) Nonself recognition is mediated by HET-C heterocomplex formation during vegetative incompatibility. The EMBO journal, 21, 4841-4850.

Sato, T., Ananda, K., Cheng, C. I., Suh, E. J., Narayanan, S. Wolfe, M. S. (2008) Distinct pharmacological effects of inhibitors of signal peptide peptidase and gamma-secretase. The Journal of biological chemistry, 283, 33287-33295.

Saupe, S. J., Kuldau, G. A., Smith, M. L. Glass, N. L. (1996) The product of the het-C heterokaryon incompatibility gene of Neurospora crassa has characteristics of a glycine-rich cell wall protein. Genetics, 143, 1589-1600. 
Scherer, M., Heimel, K., Starke, V. Kämper, J. (2006) The Clp1 protein is required for clamp formation and pathogenic development of Ustilago maydis. The Plant cell, 18, 2388-2401.

Schlesinger, R., Kahmann, R. Kämper, J. (1997) The homeodomains of the heterodimeric bE and bW proteins of Ustilago maydis are both critical for function. Molecular \& General Genetics, 254, 514-519.

Schüller, C., Brewster, J. L., Alexander, M. R., Gustin, M. C. Ruis, H. (1994) The HOG pathway controls osmotic regulation of transcription via the stress response element (STRE) of the Saccharomyces cerevisiae CTT1 gene. The EMBO journal, 13, 4382-4389.

Schulz, B., Banuett, F., Dahl, M., Schlesinger, R., Schäfer, W., Martin, T., Herskowitz, I. Kahmann, R. (1990) The b alleles of U. maydis, whose combinations program pathogenic development, code for polypeptides containing a homeodomain-related motif. Cell, 60, 295-306.

Segmüller, N., Ellendorf, U., Tudzynski, B. Tudzynski, P. (2007) BcSAK1, a stress-activated mitogen-activated protein kinase, is involved in vegetative differentiation and pathogenicity in Botrytis cinerea. Eukaryotic Cell, 6, 211-221.

Shen, H., Heacock, P. N., Clancey, C. J. Dowhan, W. (1996) The CDS1 Gene Encoding CDPdiacylglycerol Synthase In Saccharomyces cerevisiae Is Essential for Cell Growth. The Journal of biological chemistry, 271, 789-795.

Shevchenko, A., Wilm, M., Vorm, O. Mann, M. (1996) Mass Spectrometric Sequencing of Proteins from Silver-Stained Polyacrylamide Gels. Anal. Chem., 68, 850-858.

Sims, A. H., Gent, M. E., Lanthaler, K., Dunn-Coleman, N. S., Oliver, S. G. Robson, G. D. (2005) Transcriptome analysis of recombinant protein secretion by Aspergillus nidulans and the unfoldedprotein response in vivo. Applied and environmental microbiology, 71, 2737-2747.

Snetselaar, K. M., Bölker, M. Kahmann, R. (1996) Ustilago maydis Mating Hyphae Orient Their Growth toward Pheromone Sources. Fungal genetics and biology, 20, 299-312.

Snetselaar, K. M. \& Mims, C. W. (1993) Infection of Maize Stigmas by Ustilago maydis: Light and Electron Microscopy. Phytopathology, 83, 843.

Spellig, T., Bölker, M., Lottspeich, F., Frank, R. W. Kahmann, R. (1994) Pheromones trigger filamentous growth in Ustilago maydis. The EMBO journal, 13, 1620-1627.

Tabas, I. \& Ron, D. (2011) Integrating the mechanisms of apoptosis induced by endoplasmic reticulum stress. Nature cell biology, 13, 184-190. 
Tanaka, S., Brefort, T., Neidig, N., Djamei, A., Kahnt, J., Vermerris, W., Koenig, S., Feussner, K., Feussner, I. Kahmann, R. (2014) A secreted Ustilago maydis effector promotes virulence by targeting anthocyanin biosynthesis in maize. eLife, 3, e01355.

Tansey, W. P. (2001) Transcriptional activation: risky business. Genes \& development, 15, 1045-1050.

Teichmann, B., Liu, L., Schink, K. O. Bölker, M. (2010) Activation of the ustilagic acid biosynthesis gene cluster in Ustilago maydis by the $\mathrm{C} 2 \mathrm{H} 2$ zinc finger transcription factor Rua1. Applied and environmental microbiology, 76, 2633-2640.

Thon, M. R., Nuckles, E. M., Takach, J. E. Vaillancourt, L. J. (2002) CPR1: a gene encoding a putative signal peptidase that functions in pathogenicity of Colletotrichum graminicola to maize. Molecular plant-microbe interactions, 15, 120-128.

Todd, B. L., Stewart, E. V., Burg, J. S., Hughes, A. L. Espenshade, P. J. (2006) Sterol regulatory element binding protein is a principal regulator of anaerobic gene expression in fission yeast. Mol. Cell. Biol., 26, 2817-2831.

Tokuoka, M., Tanaka, M., Ono, K., Takagi, S., Shintani, T. Gomi, K. (2008) Codon optimization increases steady-state mRNA levels in Aspergillus oryzae heterologous gene expression. Applied and environmental microbiology, 74, 6538-6546.

Travers, K. J., Patil, C. K., Wodicka, L., Lockhart, D. J., Weissman, J. S. Walter, P. (2000) Functional and genomic analyses reveal an essential coordination between the unfolded protein response and ER-associated degradation. Cell, 101, 249-258.

Treier, M., Staszewski, L. M. Bohmann, D. (1994) Ubiquitin-dependent c-Jun degradation in vivo is mediated by the $\Delta$ domain. Cell, 78, 787-798.

Tsukuda, T., Carleton, S., Fotheringham, S. Holloman, W. K. (1988) Isolation and characterization of an autonomously replicating sequence from Ustilago maydis. Mol. Cell. Biol., 8, 3703-3709.

Tsvetanova, N. G., Riordan, D. P. Brown, P. O. (2012) The yeast Rab GTPase Ypt1 modulates unfolded protein response dynamics by regulating the stability of HAC1 RNA. PLoS genetics, 8, e1002862.

Turi, T. G. \& Loper, J. C. (1992) Multiple regulatory elements control expression of the gene encoding the Saccharomyces cerevisiae cytochrome P450, lanosterol 14 alpha-demethylase (ERG11). The Journal of biological chemistry, 267, 2046-2056.

Tyanova, S., Temu, T., Carlson, A., Sinitcyn, P., Mann, M. Cox, J. (2015) Visualization of LCMS/MS proteomics data in MaxQuant. Proteomics, 15, 1453-1456. 
Tyanova, S., Temu, T., Sinitcyn, P., Carlson, A., Hein, M. Y., Geiger, T., Mann, M. Cox, J. (2016) The Perseus computational platform for comprehensive analysis of (prote)omics data. Nature Methods, 13, 731.

Urban, M., Kahmann, R. Bölker, M. (1996) Identification of the pheromone response element in Ustilago maydis. Molecular \& General Genetics, 251, 31-37.

Voss, M., Schröder, B. Fluhrer, R. (2013) Mechanism, specificity, and physiology of signal peptide peptidase (SPP) and SPP-like proteases. Biochimica et biophysica acta, 1828, 2828-2839.

Wang, J.-R., Li, Y.-Y., Liu, D.-N., Liu, J.-S., Li, P., Chen, L.-Z. Xu, S.-D. (2015) Codon Optimization Significantly Improves the Expression Level of $\alpha$-Amylase Gene from Bacillus licheniformis in Pichia pastoris. BioMed research international, 2015, 248680.

Weihofen, A., Binns, K., Lemberg, M. K., Ashman, K. Martoglio, B. (2002) Identification of signal peptide peptidase, a presenilin-type aspartic protease. Science, 296, 2215-2218.

Weihofen, A., Lemberg, M. K., Friedmann, E., Rueeger, H., Schmitz, A., Paganetti, P., Rovelli, G. Martoglio, B. (2003) Targeting presenilin-type aspartic protease signal peptide peptidase with gamma-secretase inhibitors. The Journal of biological chemistry, 278, 16528-16533.

Weihofen, A. \& Martoglio, B. (2003) Intramembrane-cleaving proteases: controlled liberation of proteins and bioactive peptides. Trends in Cell Biology, 13, 71-78.

Weirauch, M. T., Yang, A., Albu, M., Cote, A. G., Montenegro-Montero, A., Drewe, P. et al. (2014) Determination and inference of eukaryotic transcription factor sequence specificity. Cell, 158, 14311443.

Welihinda, A. A., Tirasophon, W., Green, S. R. Kaufman, R. J. (1998) Protein Serine/Threonine Phosphatase Ptc2p Negatively Regulates the Unfolded-Protein Response by Dephosphorylating Ire1p Kinase. Mol. Cell. Biol., 18, 1967-1977.

Whitmarsh, A. J. \& Davis, R. J. (2000) Regulation of transcription factor function by phosphorylation. CMLS, Cell. Mol. Life Sci., 57, 1172-1183.

Willger, S. D., Puttikamonkul, S., Kim, K.-H., Burritt, J. B., Grahl, N., Metzler, L. J., Barbuch, R., Bard, M., Lawrence, C. B. Cramer, R. A. (2008) A sterol-regulatory element binding protein is required for cell polarity, hypoxia adaptation, azole drug resistance, and virulence in Aspergillus fumigatus. PLoS pathogens, 4, e1000200.

Wimalasena, T. T., Enjalbert, B., Guillemette, T., Plumridge, A., Budge, S., Yin, Z., Brown, A. J. P. Archer, D. B. (2008) Impact of the unfolded protein response upon genome-wide expression patterns, and the role of Hac1 in the polarized growth, of Candida albicans. Fungal genetics and biology, 45, 1235-1247. 
Winkler, A., Arkind, C., Mattison, C. P., Burkholder, A., Knoche, K. Ota, I. (2002) Heat Stress Activates the Yeast High-Osmolarity Glycerol Mitogen-Activated Protein Kinase Pathway, and Protein Tyrosine Phosphatases Are Essential under Heat Stress. Eukaryotic Cell, 1, 163-173.

Witte, C., Jensen, R. E., Yaffe, M. P. Schatz, G. (1988) MAS1, a gene essential for yeast mitochondrial assembly, encodes a subunit of the mitochondrial processing protease. The EMBO journal, 7, 1439-1447.

Woehlbier, U. \& Hetz, C. (2011) Modulating stress responses by the UPRosome: a matter of life and death. Trends in biochemical sciences, 36, 329-337.

Yamamoto, K., Yoshida, H., Kokame, K., Kaufman, R. J. Mori, K. (2004) Differential contributions of ATF6 and XBP1 to the activation of endoplasmic reticulum stress-responsive cis-acting elements ERSE, UPRE and ERSE-II. Journal of biochemistry, 136, 343-350.

Yi, M., Chi, M.-H., Khang, C. H., Park, S.-Y., Kang, S., Valent, B. Lee, Y.-H. (2009) The ER chaperone LHS1 is involved in asexual development and rice infection by the blast fungus Magnaporthe oryzae. The Plant cell, 21, 681-695.

Yoshida, H., Matsui, T., Yamamoto, A., Okada, T. Mori, K. (2001) XBP1 mRNA is induced by ATF6 and spliced by IRE1 in response to ER stress to produce a highly active transcription factor. Cell, 107, 881-891.

Zarnack, K., Maurer, S., Kaffarnik, F., Ladendorf, O., Brachmann, A., Kämper, J. Feldbrügge, M. (2006) Tetracycline-regulated gene expression in the pathogen Ustilago maydis. Fungal genetics and biology, 43, 727-738.

Zuker, M. (2003) Mfold web server for nucleic acid folding and hybridization prediction. Nucleic acids research, 31, 3406-3415. 


\section{Appendix}

\subsubsection{ChIPseq analysis revealed Cib1 binding in tin1-1 promoter}
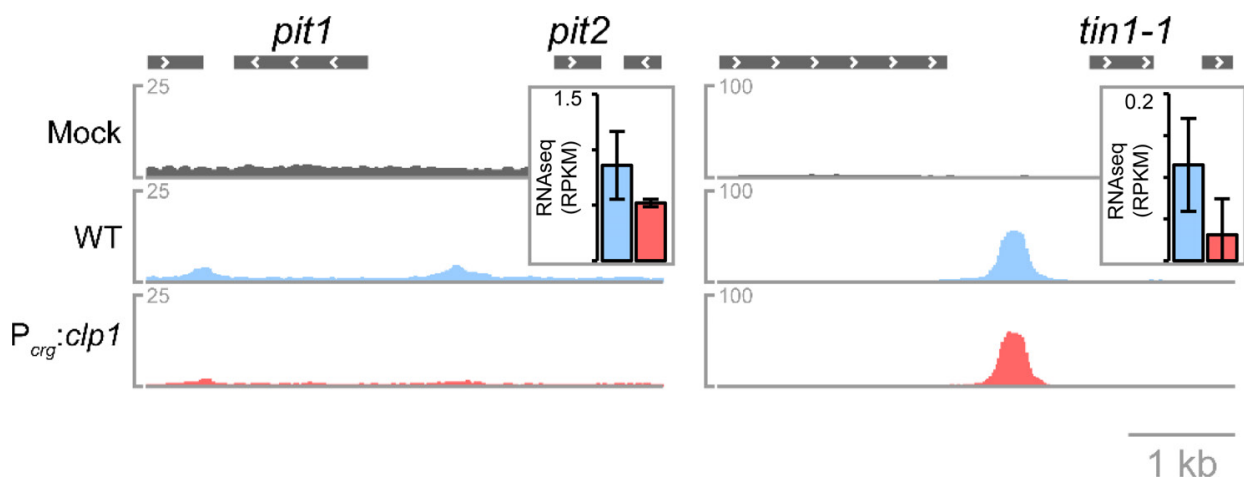

Figure 7.1: ChIPseq analysis of effector genes pit1/2 and tin1-1 in $\boldsymbol{U}$. maydis. Visualization of Cib1 binding in promoters of $U$. maydis effector genes pit1 and tin1-1 obtained by ChIPseq analysis. Strains, growth conditions and visualization of data was performed as described in chapter 3.2.3. 


\subsubsection{SPP share highly conserved motifs}

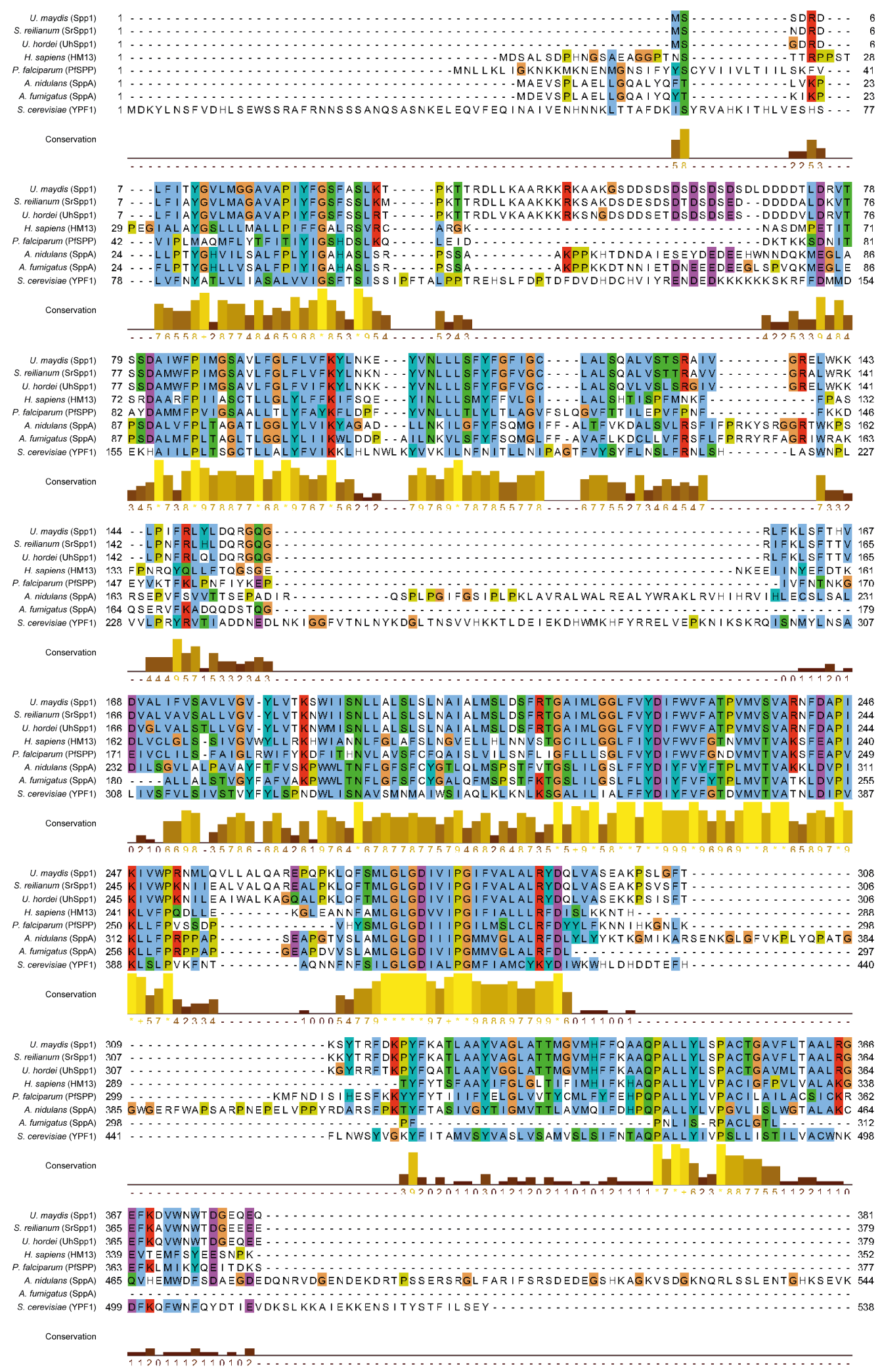




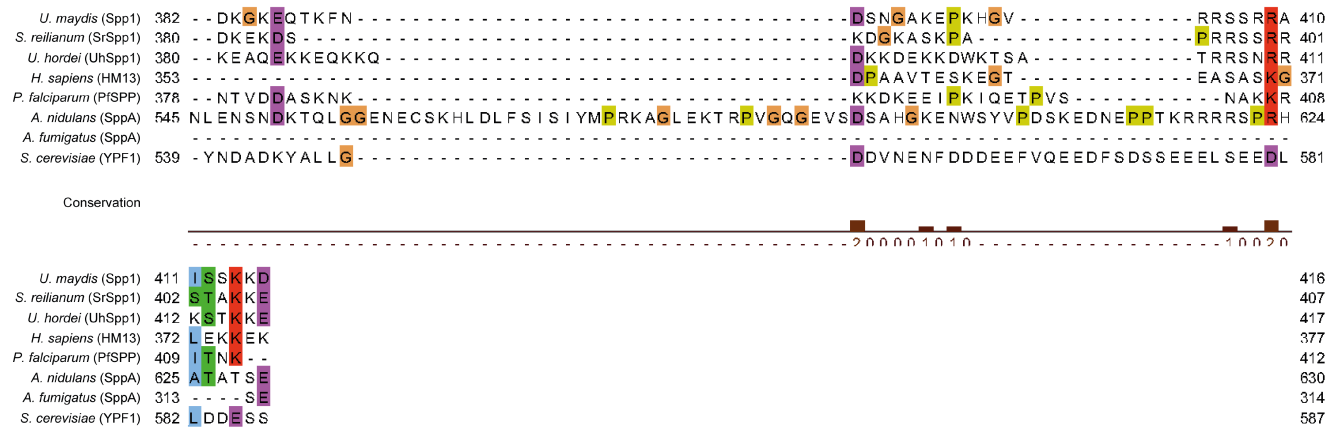

Figure 7.2: Complete multiple alignment of SPP orthologs. Protein sequences of $U$. maydis Spp1 and predicted orthologs from indicated species were aligned using the MUSCLE algorithm (https://www.ebi.ac.uk/Tools/msa/muscle) and visualized by JalView (http://www.jalview.org).

\subsection{3 $\triangle$ spp1 led to increased expression of PR3 and PR4 in planta}
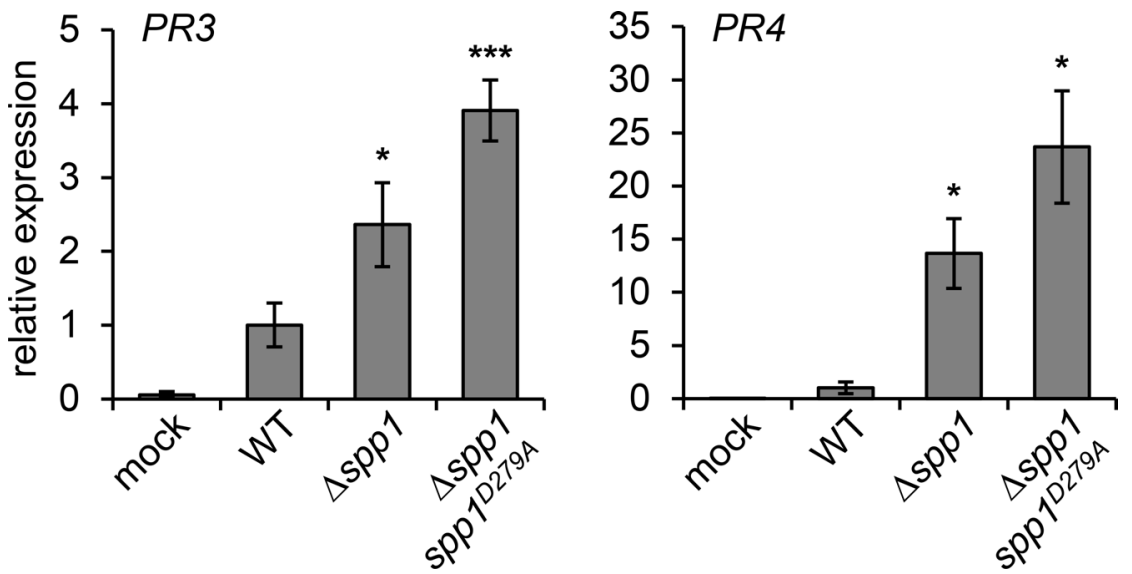

Figure 7.3: qRT-PCR analysis of pathogenesis-related plant genes of Zea mays. qRT-PCR based expression analysis of defense-related Z. mays genes in response to infection with indicated $U$. maydis strains. 7-day-old maize seedlings were used for inoculation and samples of infected leaf tissue were collected 2 dpi. Expression levels are depicted relative to plants infected with the WT and represent the mean of three biological replicates with two technical duplicates each. GAPDH was used for normalization. Additional SA responsive genes $P R 3$, $P R 4$ were tested. Error bars represent the SD. Statistical significance was calculated using Student's $t$-test. *Pvalue $\leq 0.05$ and $* * *$ P-value $\leq 0.001$. 


\subsection{4 $\Delta$ spp1 strains are not impaired in cell wall stress}

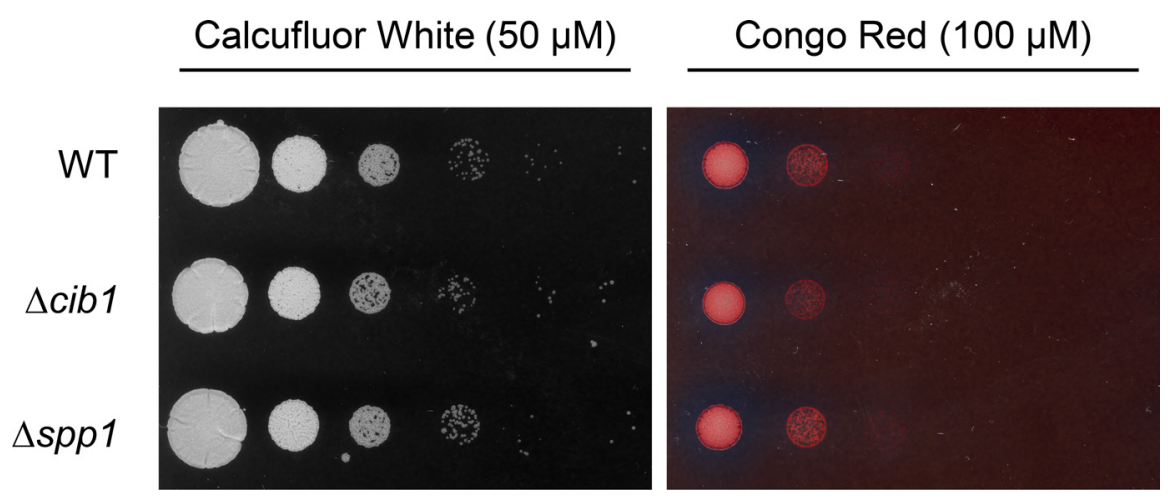

Figure 7.4: Cell wall stress assay of $\Delta$ spp1 strain. Cell wall stress resistance of $U$. maydis strain SG200 (WT) and the $\Delta$ spp1 derivative was tested by serial 10-fold dilutions of strains, spotted on YNBG solid medium supplemented with Calcofluor White $(50 \mu \mathrm{M})$ or Congo Red $(100 \mu \mathrm{M})$ as indicated. Plates were incubated for $48 \mathrm{~h}$ at $28^{\circ} \mathrm{C}$.

\subsubsection{Coomassie staining of the Cib1 protein in an SDS-polyacrylamide gel}

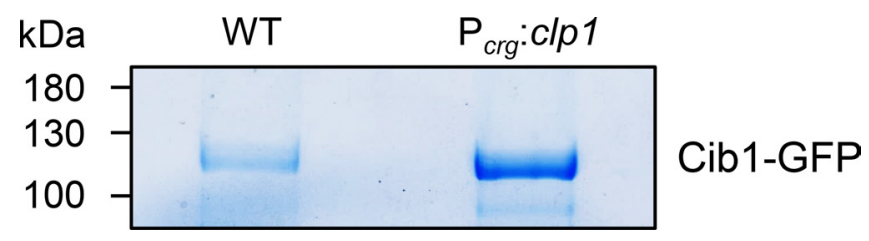

Figure 7.5: Coomassie staining of an SDS-polyacrylamide gel used for phosphosite identification of Cib1.

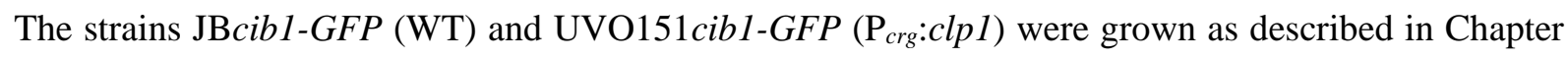
3.1.5. Proteins were extracted and Cib1-GFP was immunoprecipitated. Samples were separated by SDSpolyacrylamide gel (10\%). After separation, the SDS-polyacrylamide gel was stained with Coomassie and Cib1-GFP bands were excised and subjected to LC-MS analysis. 


\subsubsection{Files and tables}

\section{Appendix File 1: AF2_ChIPseq python scripts.zip}

ZIP compressed files. Run the python scripts in the following order:

1. peak2promoter.py

Change:

worksheetID (JB1 or UVO151)

2. peak2gene.py

Change:

worksheetID, columnExpression and xFactor according to the used expression data (JB1 or UVO151)

3. promoterScoreSum.py

Change:

worksheetID (JB1 or UVO151)

The file "ChIPseq_raw.xlsx" contains RNAseq data of chapter 3.2.1 and promoter information of all genes of $U$. maydis. Results will be automatically saved in "ChIPseq_raw.xlsx" after data processing of each script

\section{Appendix File 2: AF1_LC-MS Spp1 parameter file.xml}

Parameter file of MaxQuant v. 1.6.1.6. from Spp1-GFP immunoprecipitation with subsequent LC-MS analysis. Settings used in MaxQuant for raw data can be obtained from this parameter file.

\section{Appendix Table 1: AT1_NetPhos.xlsx}

Full list of predicted phosphosites in the Cib1 protein sequence by NetPhos-3.1.

\section{Appendix Table 2: AT2_RNAseq.xlsx}

Normalized expression of $U$. maydis genes (RPKM) and comparison between strains.

\section{Appendix Table 3: AT3_ChIPseq.xlsx}


Peaks identified by ChIPseq analysis in strain JB1cib1-3xHA.

\section{Appendix Table 4: AT4_FunCat.xlsx}

FunCat analysis of UPR core genes.

\section{Appendix Data/CD-ROM}

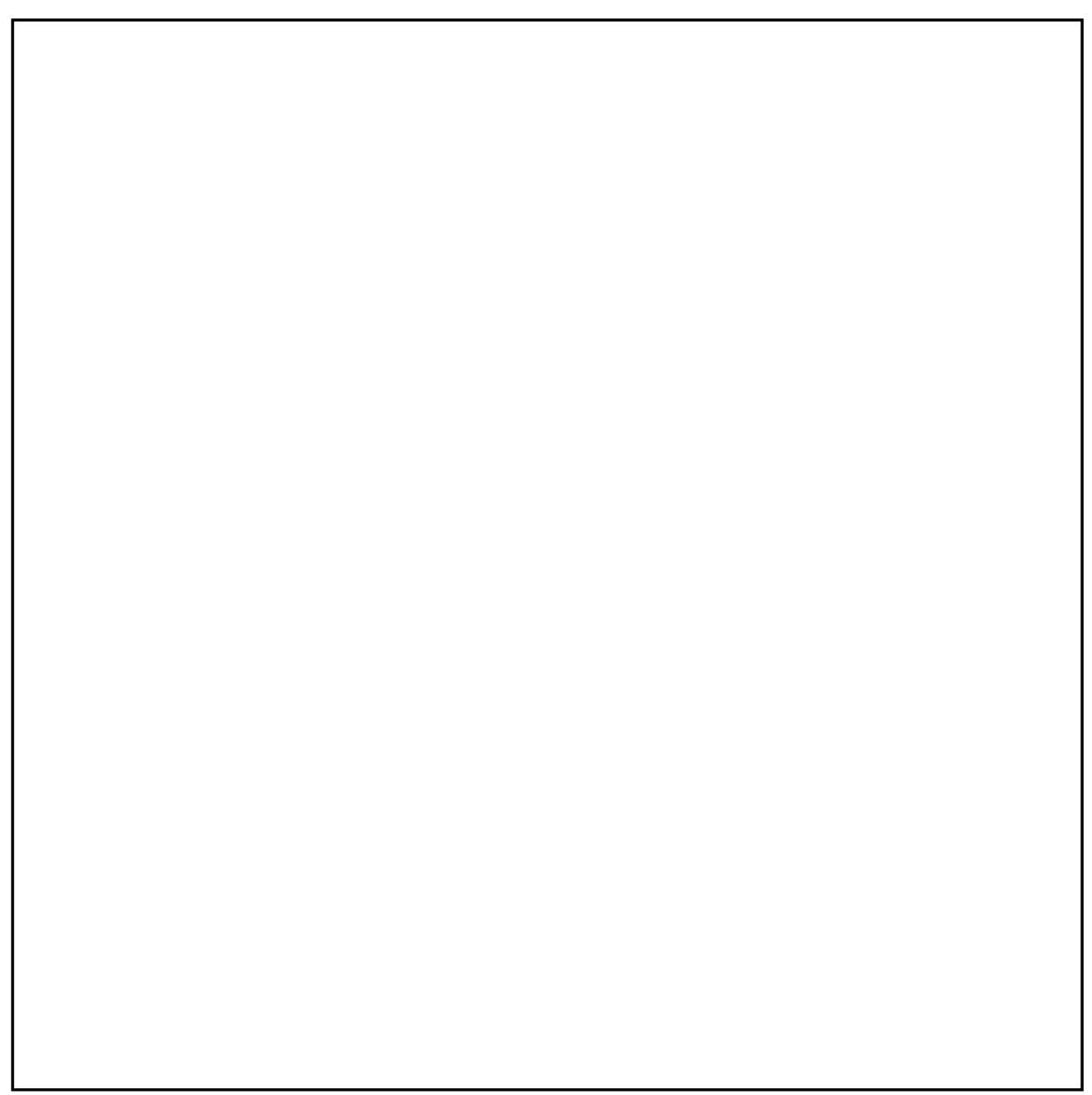




\section{List of abbreviations}

\begin{tabular}{|c|c|}
\hline Abbreviation & Description \\
\hline$\%$ & percent \\
\hline (v/v) & volume per volume \\
\hline$(w / v)$ & weight per volume \\
\hline${ }^{\circ} \mathbf{C}$ & degree Celsius \\
\hline AA & amino acids \\
\hline ATF4 & activating transcription factor 4 \\
\hline ATF6 & activating transcription factor 6 \\
\hline bbs & b-binding site \\
\hline bE & bEast \\
\hline BLASTp & basic local alignment search tool \\
\hline bp & base pairs \\
\hline bW & bWest \\
\hline bZIP & basic-region leucine zipper \\
\hline CbxR & carboxin resistance \\
\hline cDNA & complementary DNA \\
\hline ChIPseq & chromatin immunoprecipitation sequencing \\
\hline CHX & cycloheximide chase assay \\
\hline Cib1 & Clp1 interacting bZIP 1 \\
\hline Clp1 & Clampless 1 \\
\hline Clp1-ID & Clp1 interaction domain \\
\hline CM & complete medium \\
\hline C-terminus & carboxy-/COOH-terminus \\
\hline DAB & diaminobenzidine \\
\hline DIC & differential interference contrast \\
\hline DMSO & dimethylsulfoxide \\
\hline DNA & deoxyribonucleic acid \\
\hline DOX & doxycycline-based promoter shut-off assay \\
\hline dpi & days after inoculation \\
\hline DPI & diphenyleneiodonium \\
\hline DTT & dithiothreitol \\
\hline elF2b & eukaryotic initiation factor 2 \\
\hline ER & endoplasmic reticulum \\
\hline ERAD & ER-associated degradation \\
\hline E-Value & expected value \\
\hline FunCat & functional catalog \\
\hline GAPDH & glyceraldehyde 3-phosphate dehydrogenase gene \\
\hline GFP & green fluorescent protein \\
\hline HA & hemagglutinin \\
\hline $\mathrm{HCV}$ & hepatitis $\mathrm{C}$ virus \\
\hline Het-C & heterokaryon incompatibility protein \\
\hline HM13 & minor histocompatibility antigen $\mathrm{H} 13$ \\
\hline HR & hypersensitive response \\
\hline HygR & hygromycin resistance \\
\hline I-CLiPs & aspartyl intramembrane-cleaving proteases \\
\hline
\end{tabular}




\begin{tabular}{|c|c|}
\hline IGV & integrative genome viewer \\
\hline ip & iron-sulfur protein \\
\hline Ire1 & inositol-requiring enzyme 1 \\
\hline JA & jasmonic acid \\
\hline kb & kilobase pairs \\
\hline kDa & kilodalton \\
\hline LB & left border \\
\hline LC-MS & liquid chromatography-mass spectrometry \\
\hline LFQ & label-free quantification \\
\hline $\log 2 \mathrm{FC}$ & log2 fold change \\
\hline MA & magnetic agarose \\
\hline MAPK & stress-activated protein kinase \\
\hline MAST & Motif Alignment and Search Tool \\
\hline Mb & megabase pairs \\
\hline $\mathrm{mC}$ & mCherry \\
\hline MEME & Multiple Em for Motif Elicitation \\
\hline mfc & mean fold changes \\
\hline MHC & major histocompatibility complex \\
\hline mRNA & messenger RNA \\
\hline MUSCLE & MUltiple Sequence Comparison by Log-Expectation \\
\hline NatR & nourseothricin resistance \\
\hline NeoR & neomycin/geniticin resistance \\
\hline N-terminus & amino-/NH2-terminus \\
\hline OD & optical density \\
\hline ORF & open reading frame \\
\hline PCR & polymerase chain reaction \\
\hline PD & potato dextrose \\
\hline PERK & double-stranded RNA-activated protein kinase (PKR)-like ER kinase \\
\hline Phl & phosphatase inhibitor \\
\hline PhleoR & phleomycin resistance \\
\hline $\mathbf{P I}$ & proteinase inhibitor \\
\hline ps & prediction score \\
\hline PSEN & presenilin proteases \\
\hline PSM & peptide spectrum matches \\
\hline PTI & PAMP-triggered immunity \\
\hline pUPRE & predicted UPRE \\
\hline P-value & probability value \\
\hline PVDF & polyvinylidene difluoride \\
\hline qChIP & quantitative ChIP \\
\hline qRT-PCR & quantitative reverse-transcription PCR \\
\hline RB & right border \\
\hline RFP & red fluorescent protein \\
\hline RIDD & regulated IRE1-dependent decay of mRNA \\
\hline RNA & ribonucleic acid \\
\hline RNAseq & RNA sequencing \\
\hline ROS & reactive oxygen species \\
\hline RPKM & reads per kilobase million \\
\hline
\end{tabular}




\begin{tabular}{|c|c|}
\hline RPM & reads per million \\
\hline rpm & rounds per minute \\
\hline RT & room temperature \\
\hline SA & salicylic acid \\
\hline SAPK & stress-activated protein kinase \\
\hline SD & standard deviation \\
\hline SDS-PAGE & sodium dodecyl sulfate polyacrylamide gel electrophoresis \\
\hline SEM & standard error of the mean \\
\hline SPC & signal peptidase complex \\
\hline Spp1 & Signal peptide peptidase 1 \\
\hline sps & substitutions per site \\
\hline SREBP & sterol regulatory element-binding protein \\
\hline TM & tunicamycin \\
\hline TMD & transmembrane domain \\
\hline tSIM & targeted selected ion monitoring \\
\hline tss & transcription start site \\
\hline UPR & unfolded protein response \\
\hline UPRE & UPR element \\
\hline WT & wildtype \\
\hline Xbp1 & X-box binding protein 1 \\
\hline YNB & yeast nitrogen base \\
\hline$\Delta$ & delta/deletion \\
\hline$\lambda-P P$ & $\lambda$-phosphatase \\
\hline
\end{tabular}




\section{Table of figures}

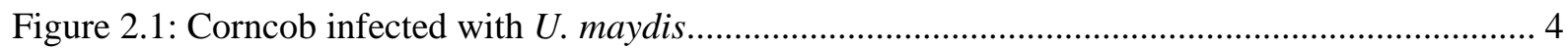

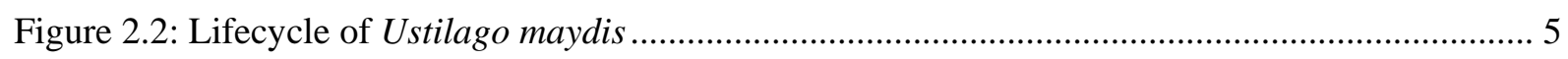

Figure 2.3: Schematic representation of the $b$-locus in $U$. maydis ...................................................... 7

Figure 2.4: Model of the transcriptional network of sexual and pathogenic development in U. maydis 8

Figure 2.5: Schematic representation of UPR pathways compete with ER stress................................. 10

Figure 2.6: Sensing of unfolded proteins via the Ire1 pathway ............................................................. 11

Figure 2.7: Model of the developmental switch initiated by the UPR ................................................ 12

Figure 2.8: Schematic representation of the SPP/SPPL domain topology and substrate processing .... 14

Figure 3.1: Induction of Clp1 during ER stress increases ER stress tolerance...................................... 17

Figure 3.2: Cib1-GFP localization is altered upon Clp1 induction .................................................... 17

Figure 3.3: Western hybridization of Cib1-GFP and qRT-PCR of $c i b 1^{s}$ in dependency of Clp1 expression 18

Figure 3.4: Clp1 expression increases Cib1-GFP protein levels ....................................................... 20

Figure 3.5: Altered phosphorylation of Cib1-GFP by expression of clp1 …........................................ 21

Figure 3.6: Schematic overview of Cib1 domains and putative phosphosites ...................................... 23

Figure 3.7: Phosphomutations of Cib1 had no impact on pathogenicity ................................................. 24

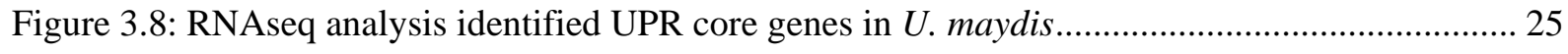

Figure 3.9: UPR core genes are enriched in functional categories with ER and UPR-related function 26

Figure 3.10: Heat map of hierarchical clustered UPR core genes...................................................... 27

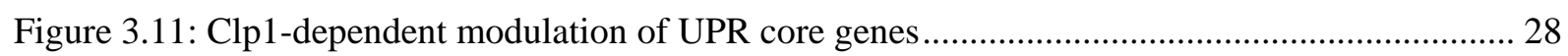

Figure 3.12: Cib1 DNA binding specificity is not altered upon Clp1 induction ..................................... 30

Figure 3.13: Visualization of ChIP peaks in known UPR target genes.............................................. 31

Figure 3.14: Comparison of promoter scores derived from ChIPseq analysis ...................................... 32

Figure 3.15: The UPR core gene UMAG_02729 is crucial for pathogenicity in U. maydis ................. 34

Figure 3.16: UPR core genes are not involved in ER stress tolerance ................................................ 36

Figure 3.17: Schematic representation of the Spp1 domain structure ................................................ 37

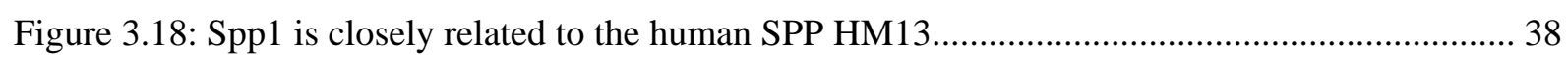

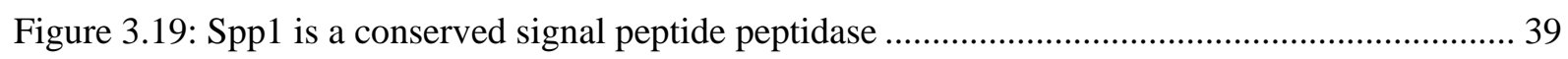

Figure 3.20: Spp1 is localized at the ER membrane and induced during ER stress .............................. 40

Figure 3.21: Filament formation is not impaired in spp1 deletion strains ........................................... 41

Figure 3.22: Deletion mutant of spp1 attenuated in growth after plant invasion .................................. 42

Figure 3.23: Spp1 function is crucial for growth in planta ................................................................... 43

Figure 3.24: Western hybridization analysis of Spp1 mutants and orthologous proteins of Spp1........ 43

Figure 3.25: Orthologous spp1 genes could recover the spp1 deletion phenotype ............................... 45

Figure 3.26: spp1 mutants elicited plant defense responses ................................................................ 46

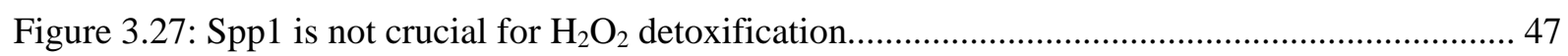


Figure 3.28: Inhibition of ROS production in planta could not recover the virulence of the $\Delta$ spp1 mutant

Figure 3.29: Strains with loss of the Spp1 function elicited strong induction of plant defense response genes during infection of Zea mays.

Figure 3.30: ERAD is dispensable for pathogenicity of $U$. maydis ...................................................... 51

Figure 3.31: Deletion of ERAD genes did not affect ER stress tolerance................................................ 52

Figure 3.32: Characterization of the $s r b 1$ deletion strain in $U$. maydis ............................................... 53

Figure 3.33: Growth of the $\Delta$ spp1 mutant is not impaired under hypoxic conditions............................ 53

Figure 3.34: Secretion of Pit2-mC is not impaired in the spp1 deletion strain ..................................... 54

Figure 3.35: Secretion of Pep1-mC, Tin2-mC and Cmu1-mC is not impaired in the $\Delta$ spp1 strain ...... 55

Figure 3.36: Genes of the fungal UPR were not upregulated in $\triangle$ spp1 strains during plant infection.. 56

Figure 3.37: Scheme of UPREs in the spp1 promoter identified by ChIPseq....................................... 57

Figure 3.38: Strains with UPRE deletions in the spp1 promoter had a reduced expression during ER

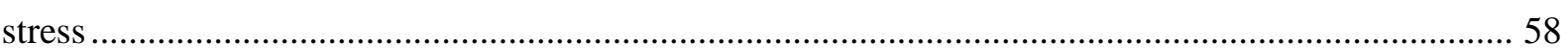

Figure 3.39: UPRE deletion mutants had a slightly reduced virulence................................................ 59

Figure 3.40: Schematic representation of predicted UPREs in promoters of spp1 and orthologous genes 59

Figure 3.41: Predicted UPREs (pUPRE) in promoters of spp1 and orthologous genes ........................ 60

Figure 3.42: Western hybridization of pull-down of Spp1-GFP and Spp1 $1^{\text {D279A }}$-GFP ........................... 61

Figure 3.43: Heat map of potential Spp1 interaction partners identified by LC-MS analysis .............. 63

Figure 3.44: UMAG_02578-GFP accumulates upon ER stress at the nucleus of $U$. maydis................ 64

Figure 3.45: Deletion mutants of UMAG_02578 have no impact on pathogenicity .............................. 65

Figure 4.1: Schematic representation of the Cib1 binding site in the spp1 promoter ............................ 76

Figure 4.2: Model of the Clp1-dependent modulation of the Cib1 function .......................................... 81

Figure 5.1: Schematic representation of the gene deletion by homologous recombination ................. 113

Figure 5.2: Schematic representation of the ectopic integration of constructs in the ip locus ............ 114 


\section{List of tables}

Table 3.1: Identified phosphosites of Cib1-GFP by LC-MS analysis ................................................... 22

Table 3.2: Top 20 candidates of promoters with the highest promoter score identified by ChIPseq.... 30

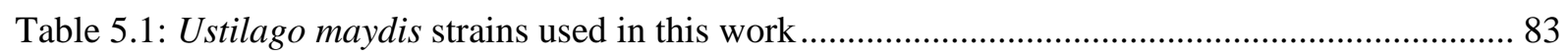

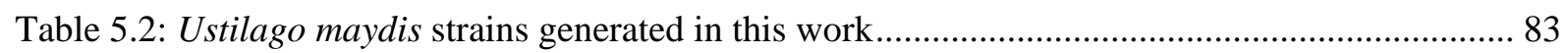

Table 5.3: Ustilago maydis strains generated in a supervised master thesis (Hach, 2018) ................... 87

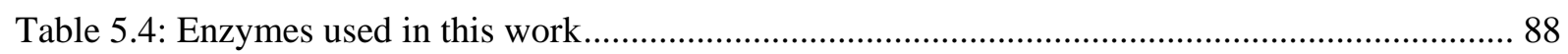

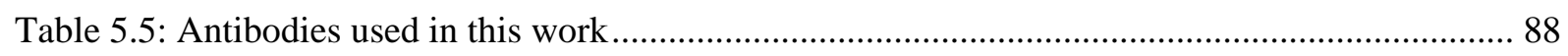

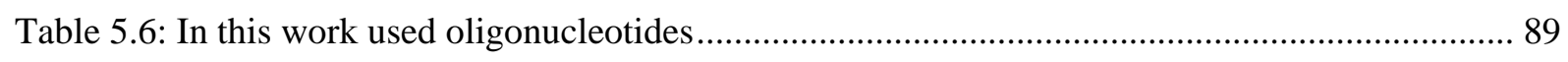

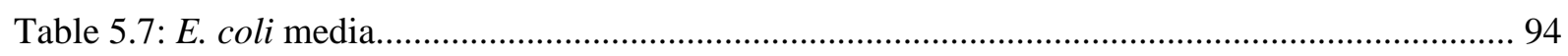

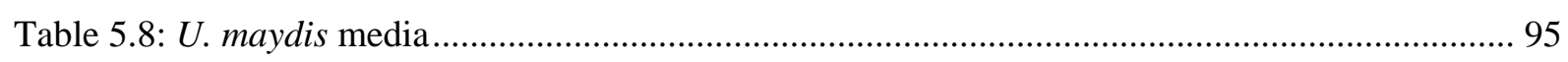

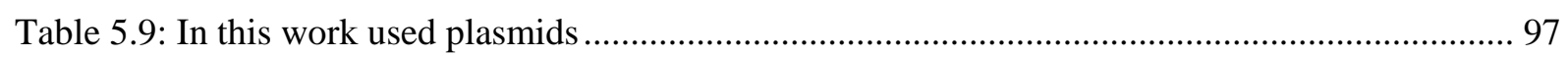

